\title{
Knowledge flows and social capital : a network perspective on rural innovation
}

Citation for published version (APA):

Arora, S. (2009). Knowledge flows and social capital : a network perspective on rural innovation. [Doctoral Thesis, Maastricht University]. Datawyse / Universitaire Pers Maastricht.

https://doi.org/10.26481/dis.20090212sa

Document status and date:

Published: 01/01/2009

DOI:

10.26481/dis.20090212sa

Document Version:

Publisher's PDF, also known as Version of record

\section{Please check the document version of this publication:}

- A submitted manuscript is the version of the article upon submission and before peer-review. There can be important differences between the submitted version and the official published version of record.

People interested in the research are advised to contact the author for the final version of the publication, or visit the DOI to the publisher's website.

- The final author version and the galley proof are versions of the publication after peer review.

- The final published version features the final layout of the paper including the volume, issue and page numbers.

Link to publication

\footnotetext{
General rights rights.

- You may freely distribute the URL identifying the publication in the public portal. please follow below link for the End User Agreement:

www.umlib.nl/taverne-license

Take down policy

If you believe that this document breaches copyright please contact us at:

repository@maastrichtuniversity.nl

providing details and we will investigate your claim.
}

Copyright and moral rights for the publications made accessible in the public portal are retained by the authors and/or other copyright owners and it is a condition of accessing publications that users recognise and abide by the legal requirements associated with these

- Users may download and print one copy of any publication from the public portal for the purpose of private study or research.

- You may not further distribute the material or use it for any profit-making activity or commercial gain

If the publication is distributed under the terms of Article $25 \mathrm{fa}$ of the Dutch Copyright Act, indicated by the "Taverne" license above, 


\section{Knowledge Flows and Social Capital}

A network perspective on rural innovation

Saurabh Arora 
(C) 2009 Saurabh Arora ISBN 9789052788074

Published by Universitaire Pers Maastricht

Printed in the Netherlands by DataWyse Maastricht 


\title{
Knowledge Flows and Social Capital
}

\section{A network perspective on rural innovation}

\author{
PROEFSCHRIFT
}

ter verkrijging van de graad van doctor aan de Universiteit Maastricht, op gezag van de Rector Magnificus, Prof. mr. G.P.M.F. Mols, volgens het besluit van het College van Decanen,

in het openbaar te verdedigen op donderdag 12 februari 2009 om 16.00 uur

door

Saurabh Arora

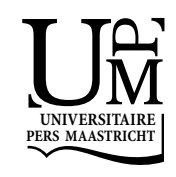


Promotor:

Prof. dr. Robin Cowan

Beoordelingscommissie:

Prof. dr. Arno Riedl, voorzitter

Prof. dr. Alan Kirman, GREQAM, Université d'Aix Marseille III

Prof. dr. Luc Soete 
For my mother and father 


\section{Acknowledgements}

Over the course of the last seven years, I have accumulated many debts. So there are a lot of people to thank and I hope I will not forget anyone. But if I do, I hope to be forgiven.

Let me start by thanking my promotor and supervisor, Robin, without whose support and guidance this thesis would have remained incomplete forever. Many other people at MERIT have been very helpful, in particular Eveline, Corien, Wilma, Ad, Silvana, Marc and Zajia. Among the academic staff, I am grateful to Eddy Szirmai, Geert Duijsters, René Kemp, Ionara Costa and Anthony Arundel for insightful comments and discussion. Anthony also saved me from the street on at least two occasions by letting me stay in his house for several months. Some people at the old INTECH helped with comments or otherwise. In particular, I thank Yvonne, Martin, Lynn Mytelka and Keith Smith.

I thank the three members of my committee, Arno Riedl, Alan Kirman, and Luc Soete, who were generous to read and provide valuable comments.

In India, Andy Hall and others at CRISP, provided much-needed initial hospitality and institutional support. People at CSA in Hyderabad always had time for my neverending questions: in particular, I thank Dr Ramanjaneylu, and Kavitha Kuruganti who spent hours explaining the intricacies of their NPM work to me. Mr Venumadhav, Ramesh, G.C. RaviKumar, Nirmala and others from SECURE were welcoming and helpful in Palvancha and Punukula. Poorna Chandra and Usha translated the questionnaires into Telegu. Poorna also provided indispensable help in conducting the interviews. I thank all the hospitable and warm people of Punukula who never hesitated in answering my often-intrusive questions. I fondly remember conversations with Hemla Naik, Eerla Danamma, Chikkula Venkanna, Challa Ramachandru, Banoth Kausalya, Bommanaboina Savitri, Chakrala Yalamanchi, Chakrala Nageshwar Rao, and Sheikh Khalil.

At the technical university in Eindhoven where I work now, colleagues and friends have been supportive and encouraging in the final year of this journey. I owe my deepest gratitude to Marianne Jonker, Saskia Repelaar, Henny Romijn, Annelies Balkema, Önder Nomaler, Arianna Martinelli and Johanna Ulmannen. Henny also translated the summary into Dutch and Annelies provided some critical help in designing the cover.

I am grateful to Simryn Gill and the Queensland art gallery for letting me use a photograph of Gill's Forking Tongues installation on the cover.

Friends of mine who started their PhD's with me were great company in those early days in Maastricht. I enjoyed many a great evening of food, wine and discussion with Paco, Fulvia, Tet, Marcia, Ngoc, Sandra, Victoria, Abraham and Ranjitha. I made some other wonderful friends in Maastricht over the years, who often provided me with dinner and shelter, and shared beers at the end of another frustrating day filled with computer codes that didn't work. Life as an ageing student would have been 
unbearable without their company. So thank you Bulat, Jos, Julia, Kirsten, Müge, Saeed and Tomasso for putting up with my moods and rants.

Finally, I thank UNU-INTECH (now called UNU-MERIT) and the Economics Department of Maastricht University for financial support.

\section{Saurabh Arora}

Eindhoven, January 2009 


\section{Contents}

List of Tables $x$

List of Figures $x i$

1. Introduction 1

1.1 Outline of the thesis 9

Part I: Technological interdependence and evolving networks 12

2. Technological interdependence and knowledge flows 13

2.1 Mechanisms of technological interdependence 13

2.1.1 Technology succession and interdependence 16

2.1.2 Horizontal interdependence in complex products and technology systems 17

2.1.3 Generic technologies $\quad 19$

2.1.4 Vertical relationships $\quad 21$

2.2 Temporal aspects of interdependence: gradualism and discontinuities 23

2.2.1 Gradualism and persistent technological interdependence 24

2.2.2 Technological discontinuities $\quad 26$

2.3 Structure of technological interdependence 30

2.3.1 Hierarchy in systems: core and periphery 30

2.3.2 Circular flow of goods and knowledge: a structural 'tableau' for economic and technological interdependence 37

3. The growth of knowledge and complexity in an evolving network model of technological innovation $\quad 54$

3.1 Models of technological evolution $\quad 55$

$\begin{array}{ll}3.2 \text { Model } & 60\end{array}$

3.2.1 Model dynamics 61

3.3 Simulation results $\quad 63$

3.4 Simulation Experiments: Different Core Structures $\quad 71$

$\begin{array}{ll}\text { 3.4.1 Core-strength } & 71\end{array}$

3.4.2 Number of knowledge types mutated in one time-period 75

3.5 Discussion $\quad 77$

Part II: Social interactions and social capital 79

4. Introduction: networks of a rural community $\quad 80$

4.1 The community in focus $\quad 80$

4.2 The story of pest control in the village 83

5. Network data and approach used for data collection 88

5.1 Approach for collecting data on knowledge-flows in the village 90

5.2 Business network data 93

5.3 Collection of social network data 95 
5.4 Measuring innovation capacity: knowledge diffusion and experimentation $\quad 97$

6. Innovation capacity of farmers and the knowledge network. 99

6.1 Agricultural innovation: a brief overview 100

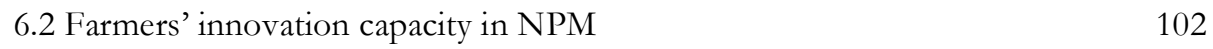

$\begin{array}{ll}\text { 6.3 The knowledge network } & 107\end{array}$

6.4 Discussion 112

6. The farmers' escape from pesticides and debt 115

7.1 The business network: accessing agricultural inputs 115

7.2 The dominant core in the business network 117

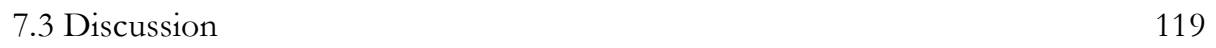

8. Socio-political events and social network structure $\quad 120$

8.1 Village India and social networks 121

8.2 Collective action and media success 126

8.3 Power, influence, centrality, and brokerage $\quad 128$

$\begin{array}{ll}\text { 8.3.1 Power and influence in a social network } & 128\end{array}$

$\begin{array}{ll}\text { 8.3.2 Brokerage and social capital } & 132\end{array}$

8.4 Community leaders in the social network and group action 138

8.5 The rift in the face of unity 142

8.6 Conclusions 143

9. Embeddedness of knowledge flows in social networks 145

9.1 Embeddedness of economic and technological activities, and social capital 146

9.2 Measures of structural embeddedness 153

9.3 Embeddedness of knowledge flows in the local innovation system 157

9.4 Social embeddedness of knowledge flows in the community 170

9.5 Discussion 173

9.6 Conclusions 176

10. Summary and conclusions 179

10.1 Summary of the results 179

10.2 Implications for rural development interventions 184

References 188

Appendix A 206

Survey Questionnaires 206

Appendix B 215

Different types of centrality measures $\quad 215$

Appendix $C \quad 216$

Bigger core in Punukula's social network $\quad 216$

Appendix D 219

Appendix to Chapter 9

Summary in Dutch 


\section{List of Tables}

4.1 Distribution of land holdings (owned and cultivated) in the village 81

6.1 Pest control technology used by the 72 NPM farmers during 2005-6 105

6.2a Crops grown by the 72 NPM farmers in Punukula in kharif 2005-6 106

6.2b Crops grown by farmers in Punukula: data on 152 households 106

6.3 Knowledge network members with high degree centrality 108

8.1 Caste and land distribution in Punukula $\quad 126$

8.2 The set of ten most central individuals in Punukula 131

8.3 The set of Punukula inhabitants with high brokerage potential 136

8.4 The list of prominent people in Punukula 139

9.1 Embeddedness and social capital for knowledge in the local innovation system 159

9.2 Regression of the knowledge flow network on the social network of the village 160

9.3 Individual embeddedness of incoming knowledge links in direct social ties 162

9.4 Individual embeddedness of outgoing knowledge links in direct social ties 163

9.5 Embeddedness of knowledge flows in the business network for pest control 166

9.6 Regression of the knowledge flow network on the business network 166

9.7 Individual embeddedness of incoming knowledge links in direct business network $\begin{array}{ll}\text { for pest control } & 167\end{array}$

9.8 Individual embeddedness of outgoing knowledge links in direct business network $\begin{array}{ll}\text { for pest control } & 169\end{array}$

9.9 Embeddedness and social capital for knowledge in Punukula only 171

9.10 Regression of the knowledge network on social network of the village 173

C.1 List of 15 people with the highest closeness centrality 216

C. 2 List of 15 people with the lowest network constraint values 216

C.3 Members of the social network core of size $20 \quad 217$

D.1 Embeddedness of incoming knowledge links in indirect social ties 219

D.2 Embeddedness of outgoing knowledge links in indirect social ties 221

D.3 Individual embeddedness of incoming knowledge links in direct social ties in $\begin{array}{ll}\text { Punukula only } & 222\end{array}$

D.4 Individual embeddedness of outgoing knowledge links in direct social ties 223

D.5 Individual embeddedness of incoming knowledge links in indirect social ties in $\begin{array}{ll}\text { Punukula only } & 223\end{array}$

D.6 Individual embeddedness of outgoing knowledge links in indirect social ties 224

D.7 Individual embeddedness of incoming knowledge links in direct business network of all transactions except credit 226

D.8 Individual embeddedness of outgoing knowledge links in business network 228

D.9 Network level embeddedness of knowledge flows in the business network of all transactions (direct and indirect) 


\section{List of Figures}

2.1 A simple idealized hierarchical system 14

2.2 Core-periphery structure in a small network 35

2.3 Comparison between a core-periphery graph and one with unconnected high-centrality nodes 36

2.4 Circular-flow diagram depicting the flow of money and values in an economy 38

2.5a Diagrammatic representation of flow of money from the public treasury 39

2.5b Diagrammatic representation of flow of rents to a landlord from his tenants 40

2.6 Two types of cybernetic systems 45

2.7 Circular process of three inventions in aluminum production 49

2.8 Circular flow of benefits between three different innovations 52

3.1 Simulation results showing the three phases in the evolution of the
technology system

3.2a An example of the graph structure in fully constructed system 66

3.2b The same system as in 3.1a after a discontinuity 67

3.3a A cycle of three nodes as the core before enhancement 68

3.3b The three-cycle core is enhanced to a core of four nodes 68

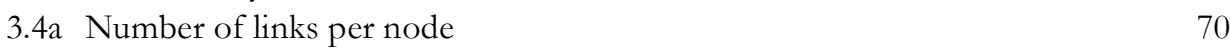

3.4b Average weight over all links in the system 70

3.5 System robustness with a complete core, a simple cyclic core and a cyclic core with two additional internal links $\quad 73$

3.6 A system constructed around a four node core with two additional internal links $\quad 73$

3.7a Discontinuity in a system with a four node cyclic core with one internal link through introduction of a stronger five node cycle with two additional links

3.7b The new cyclic structure introduced is assimilated into a constructed system with a stronger core

3.7c Discontinuity caused by the introduction of a cycle of equal strength that is downstream from the existing core

3.8 Effect of the number of types mutated in one time period on system $\begin{array}{ll}\text { behaviour } & 76\end{array}$

6.1a Total number of NPM adoptions in Punukula over time 103

6.1b Percentage of NPM adoptions each year 103

$\begin{array}{ll}\text { 6.2 Punukula's knowledge network } & 109\end{array}$

6.3 All cycles in the knowledge network of Punukula 111

6.4 The strongest two cores in the knowledge network 112

7.1 Punukula's combined business network for credit, crop sale and farm-inputs 116

7.2 The farmers' network for procuring farm-inputs and credit 116

$\begin{array}{lll}\text { 7.3 The farmers' network for selling their crop } & 117\end{array}$

7.4 Structure of the dominant core in the business network 118 
8.1 Caste-based distribution in Punukula's social network

8.2 Punukula's social network, people with high closeness centrality represented as bigger nodes

8.3 People with high network constraint represented in bigger nodes in Punukula's social network

8.4 The structure of connections in the group of prominent people identified using closeness centrality and network constraint measures

8.5 The final dominant core formed of ten Punukula residents

9.1a An example of two simple network structures under comparison

9.1b The first permutation of the adjacency matrix of graph (ii)

9.1c The second permutation of the adjacency matrix of graph (ii) 156

9.2a Knowledge flows embedded in the social network 159

9.2b Friendship and kinship ties overlaid on the knowledge network 160

9.3 Knowledge flows in the business network 167

9.4 Knowledge flows embedded in the social network of the village community only

C.1 Structure of links among the members of the social network core of size 20218 


\section{Introduction}

Continued economic growth depends on technological innovation and the underlying accumulation of knowledge. However, the successful development of new technologies and their post-adoption impact on productivity growth require parallel changes in complementary technologies and socioeconomic organization. This implies that technological change must be seen in the context of complex socio-technical systems. Coherence in such multi-technology, multi-actor systems implies two things: First, that changes in one technology do not impede but rather aid the innovation process in other technologies; changes in the interdependent technologies positively reinforce each other. Second, that decentralised action by relevant networks of actors promotes the development and use of desired technologies through successful knowledge sharing. The latter aspect of coherence requires an effective combination of different forms of social capital possessed by individual actors to make the innovation project sustainable.

Individual technologies are always embedded in larger multi-technology systems. Technological interdependence within and between these systems plays a central role in directing the dynamics of the innovation process (Rosenberg 1982; Tushman and Rosenkopf 1992). This is particularly apparent today when technological systems have become highly complex: made up of intricate networks of coupled technologies. As the philosopher Langdon Winner (2002) has warned, in such tightly interdependent systems, a small failure in one of the components can cause the collapse of the entire system. Consider the recent Internet 'blackout', which affected more than 75 million people in South Asia and the Middle East, only because two submarine cables were snapped by an unwieldy ship in the Mediterranean (The Guardian, February 1 2008). Ripples of the blackout were felt throughout the world as firms in the US and Europe failed to reach their business contacts such as outsourced call centres and software developers in India, Egypt, Pakistan, and Dubai. Commenting on the blackout, ICT experts echoed Winner in pointing to the fragility of our densely interconnected technological systems.

This example shows that the inherent complexity in technological systems may cause fragility and threats to sustainability of complete and stabilised systems made up of fully developed technologies. In addition, such complexity must be acknowledged in the process of development of new technological systems. There are two ways in which this latter complexity emerges: first, individual technological innovations are generally novel combinations of existing inventions (Usher 1954). Second, the newly developed components of a system must fit with each other for a system to fulfill its functions. Similarly, the successful introduction of a new technology into an existing system requires that modifications be made, both in the new technology and the existing system-components to which it gets connected. Failure to acknowledge the importance of these systemic interconnections has, in the past, resulted in socially 
costly technical change (nuclear power, or numerical control automation in the US: Noble 1984; Cowan 1990).

In order to grasp the complexity of systemic technical change, economists have identified and analysed a set of key technologies known as general-purpose technologies (David 1990; Bresnahan and Tratjenberg 1995; Helpman 1998; Freeman 2001). Sustained economic growth is strongly correlated with the economy-wide diffusion of general-purpose technologies (GPTs) such as the steam engine or the computer. However, such widespread diffusion often occurs slowly, over the course of several decades largely because the successful introduction of any major new technology involves significant changes in the technologies comprising an existing system and the organization of factories where they are used. For example, to utilize the unit drive electric motor in the first few decades of the $20^{\text {th }}$ century, extensive modifications were required in existing factories where manufacturing technologies were run on the group drive system of power transmission suited to water- or steampower (David and Wright 1999). ${ }^{1}$ The focus of the GPT studies by and large has been on this gradual (post-innovation) diffusion of the GPTs, accompanied learning, and the resulting impact on productivity growth. Little emphasis has been placed on the interdependencies in the pre-diffusion, developmental phase wherein the knowledge that underpins these technologies is created, and the links that make them generalpurpose are spread through the socio-technical system.

The pre-diffusion construction phase of technologies has been extensively studied by sociologists and historians of technical change interested in modifications and realignments that are common and indeed possible before a technology has stabilised. To deal with the complexity of technology development, these studies have developed different approaches including the large technological systems approach developed by Hughes (1987), the actor-network synthesis of Callon (1987), and the concept of sociotechnical ensembles due to Bijker (1995). These and related studies (for example, Latour 1996; and Mackenzie 1996) demonstrate that technological research and development does not follow a 'natural trajectory' or internal technical logic, or possess an autonomous technological momentum. According to this view, technological innovations are socially constructed and sustained, and social relations are shaped by technological and economic factors. These social constructivist studies

\footnotetext{
1 The first electric motors in these factories were often used to run a group of related machines, just like with water- or steam-power, instead of the more efficient unit drive system of powering each machine with its own electric motor (David and Wright 1999: 23-25). Only through decentralised learning by electrical engineers and other skilled personnel in different locations over decades, were the factories gradually able to switch to the unit drive system. Eventual benefits of using the latter system included zero investments for a prime-mover in a factory, cheaper factory construction as lower structural strength is required to use individually powered machines rather than the heavy transmission equipment associated with water- or steam-power, more flexibility in the operation of the factories such as better speed control made possible on individual machines, and benefits due to the modularity of the new system as only a small section of factory now needed to be shut down for maintenance/repair (David and Wright 1999: 25).
} 
have provided valuable empirical insights into the wider systemic interactions between different social groups (such as workers, engineers, scientists, users, regulators, and industry organizations) and the technologies they collectively shape and are in turn shaped by. They force us to consider that development of individual technologies is embedded in a set of social networks of actors. These networks not only allow effective sharing of knowledge but may also act as conduits for socio-political support, trust, and conviviality (and serve as the field on which competitions between different technologies, or visions of a technology, are played out).

In this dissertation, I develop an alternate way to interpret, and grapple with, the complexity of socio-technical systems, both while they are developing and when stabilised systems come under threat from newer competing technologies. In particular, I view a socio-technical system, and its network of underlying knowledge types, as having a core-periphery structure. The structure is not pre-defined, but rather emerges from technological and social interactions. Once can imagine a generic sort of history wherein a new system is 'formless' at an early stage of development when different new technologies (or versions of a technology) are tried out. During this nascent phase, there is no clear structure in which different parts of the system are organized. The emergence of a core brings the system out of its nascent experimental phase, launching a coherent phase of knowledge accumulation around a small number of core elements. Elements in the core are connected to each other in a positive feedback loop: changes in one core element send reinforcing signals to other core elements. This positive reinforcement makes the core self-sustaining and gives it the dynamism to support innovation in peripheral (complementary) elements of the system, which are directly or indirectly connected to one of the core elements. Thus, as a result of this coherence, changes in one technology do not impede, but aid innovation in other interdependent technologies and make the entire system 'dynamically sustainable'.

The coherent core in a technological system is similar to the 'hard core' of a scientific research programme, that is a sequence of theories and a heuristic principle guiding the direction of research, highlighted by Lakatos (1978). The hard core of a programme is immune to falsification and surrounded by a "protective belt" of 'auxiliary hypotheses' which "bear the brunt of tests and adjusted and re-adjusted, or even completely replaced, to defend the thus-hardened core." (Lakatos 1978: 48). Similarly, in an evolving technological system, selection pressures first hit the peripheral components which get modified or replaced while the core remains intact. However, in a stabilised or mature system, the possibilities for further knowledge accumulation are limited and sustaining the innovative dynamism of the core and periphery becomes difficult. Lakatos observes similar stagnation in degenerating research programs which fail to produce any new predictions and expend a lot of effort in explaining away the anomalies. Such a research programme in science (and technology) comes under threat from a new competing programme, which replaces an existing mature programme only if it is stronger (or more 'progressive') than the latter. 
Here strength is determined by the novel theoretical predictions produced by the research programme ("theoretically progressive"), and corroboration of the predictions using empirical data ("empirically progressive"). These theoretical and empirical results are produced according to the research programme's heuristic principle rather than by dealing with an anomaly through ad hoc adjustments to the programme (Lakatos 1978: 179). The eventual replacement by the newer research programme or technological system is generally referred to as a revolution or paradigm shift. ${ }^{2}$ Although technological systems are distinct from scientific research programmes in a number of ways, the hard core-auxiliary hypotheses framework of Lakatos corresponds well with the theoretical framework developed in this thesis for understanding the evolution of a technological system. Akin to Lakatos' arguments about competition between rival research programmes, the survival of an existing technological system when faced with competition from a rival system depends on the strength of its core and the protection and support it receives from the periphery.

Returning to arguments of the social constructivists: the development of a technology, apart from being shaped through interactions with other interdependent technologies, is driven by a number of socioeconomic actors including firms (or farmers in the case of agriculture), engineers, scientists, policy-makers, regulators, and end-users. Together these actors (with embodied knowledge and skills) and their interactions; the societal institutions that govern their behaviour; and the technologies they develop and use may be said to constitute a system of innovation (SI).

In the knowledge sphere of an innovation system, the multiple knowledge types underlying most modern technological innovations, often originate in different branches of science and engineering, such as microelectronics, software programming, and mechanical systems in the case of $\mathrm{CNC}$ machine tools. These knowledge types routinely interact with each other in the construction of new technologies. ${ }^{3}$ The necessary expertise in the different interdependent (complementary and otherwise interrelated) knowledge types is distributed across multiple actors, necessitating interaction among the actors. In addition, the users of a technology are often important contributors in the innovation process, sometimes as the most important sources of new innovations (von Hippel 1988). Then, collective learning through the exchange of knowledge in systems of innovation or communities of practice (CP) forms the basis of innovative activity. Innovative performance of the actors and eventual shape of a technology are functions of the structure of the knowledge (-sharing) network of an innovation system (or a community of practitioners). There are three primary reasons why knowledge networks are critical for learning, innovation and innovative performance,

\footnotetext{
${ }^{2}$ Note the reference to Kuhn's (1970) analysis of normal science and revolutions through shifts in scientific paradigms and to Dosi's (1982) adaptation of Kuhn's ideas for studying technological development by using the concepts of technological trajectories and paradigms.

3 The knowledge types relate to the use, design, and production of technological artefacts on the one hand, and more basic knowledge of principles and facts on the other.
} 
1. Innovation is generally a result of new combination of diverse knowledge types that must positively feed into each other in order to provide a coherent whole. ${ }^{4}$ This is as true for complex innovations in bio-pharmaceuticals as it is for Watts' steam engine. The latter for example required critical input from the knowledge of machine tools to drill cylinder bores of desired accuracy (Rosenberg 1976).

2. Diverse knowledge types which constitute technological systems ordain that no single actor can possess expertise in all knowledge types. Interaction between actors possessing expertise in interdependent technologies is necessary not only for knowledge sharing but also for the regular functioning of a system.

3. Close interaction between actors is also critical for the transfer of tacit knowledge between a) the 'experts'; b) experts and the users; and c) among the users (von Hippel 1988). Tacit knowledge is believed to be harder and slower to acquire than its codified counterpart. Transfer of tacit knowledge, generally requiring extensive faceto-face interactions, is facilitated by close social relations (Nelson and Winter 1982; Hansen 1999; Cowan et al. 2000).

Thus the sharing or exchange underpinning collective learning is not restricted to the knowledge sphere alone. Other forms of economic and non-economic exchanges in networks of social relations are believed to provide the substratum on which the interactive learning takes place, due to the tacitness of some knowledge (see the third point above). This importance of social capital for technological learning, and resulting innovation, is underlined in the prolific literature on SIs and CPs (see for example, Edquist and Johnson 1997; Lundvall et al. 2002 on the former; and Lave and Wenger 1991; Brown and Duguid 1991 on the latter). ${ }^{5}$

However, the literature generally assumes that networks of social interactions simply double as knowledge networks i.e., the same links that transmit trust and conviviality are assumed to transfer knowledge as well (in addition to studies on SI and CP cited above, see for example, Uzzi 1996; Inkpen and Tsang 2005; and Bond III et al. 2008). In this thesis, I investigate the extent to which this assumption of equating social ties to knowledge flows is justified. And if the assumption turns out to be mistaken, do we have to re-think the nature and importance of social capital for learning and innovation in socio-technical systems?

Even in the wider literature on the economics and sociology of networks, the content of links takes the backseat while network structure (the latter simply equated to social capital) drives analytical explanations (see Krippner 2001 for a critical review).

\footnotetext{
4 This statement is widely believed to be applicable only to radical innovations, as opposed to incremental innovations. However, as I argue in more detail in chapter 3, despite its unitary appearance a radical innovation may in fact be a combination of many incremental innovations. It may be treated as a gradual evolutionary process involving many incremental steps (Usher 1954; Silverberg and Verspagen 2004).

5 This social capital is generally translated as trust and common vision internally in the community or innovation system and a common united face showed to the outside world. In addition, of course it is believed to facilitate the transfer of tacit knowledge, as noted earlier.
} 
The different contents of social relationships are either clubbed together as a single pooled 'social tie', or presented as something unlike market transactions that are by definition anonymous and non-social. ${ }^{6}$ As a result, the content that flows as the 'social' through social networks often remains unclear. In this thesis, I attach specific meanings to the social in three ways: first, close kinship and friendship ties that transmit socio-political support, trust and conviviality; second, ties that transmit problem-solving knowledge; and third, ties that are used for exchanging goods (farminputs, credit, and crops produced by farmers). The three different networks focus on a single agrarian village called Punukula in Andhra Pradesh, India, where a technological transition from pesticides to non-pesticidal management (NPM) of crops was attempted in the last decade. I analyse the structures of the three networks using the core-periphery analogy and the simulation results on competition between rival systems based on core-strength from part I of the thesis.

Based on the foregoing discussion, one may naturally ask why is it critical to distinguish between different types of social capital? To answer this question, we must first develop a clear understanding of what social capital means. Following Bourdieu (1986), I define social capital as benefits that individuals derive from membership in social networks of contacts (acquaintances and close kin/friends). Here social capital has two essential components: first, membership in social networks and second, the economic and non-economic benefits that can be derived out of such membership. The first is a structural issue which can be understood by analysing an individual's position in the relevant social network. Studying the second component of social capital requires one to observe the content of the links in the social network. ${ }^{7}$

The first structural feature of social capital has been widely studied by economic sociologists and often equated to social capital itself (see for example, Coleman 1988 on closure; and Burt 1992; 2000 on structural holes). A parallel analysis of the second component of social capital, the quantity and quality of resources available or accessed

${ }^{6}$ There are other problems with the concept of social capital. For example, since the mid 1990s social capital has become a cause and an effect of anything virtuous happening to an individual, group or even a society as a whole: so it is the cause and effect of beneficence at multiple levels. This and other confusions, in interpreting and understanding social capital, precipitated after the work of Robert Putnam and colleagues (1993). More details in Chapter 8 of the thesis.

${ }^{7}$ One may ask why spend so much time and effort in mapping networks with different link-contents when one can simply regress profits or other economic/non-economic benefits on network structures. These regressions and correlations, if performed using methods that circumvent the positive correlation problem associated with network data, can indeed produce useful results and have formed the mainstay of the sub-discipline of economic sociology (see for example the seminal work of Burt 1992; Uzzi 1996; and Granovetter 2005 for a succinct review). But the statistical results, by themselves, do not tell us which of her multifarious social ties did an actor use to secure specific economic benefits. In addition, no insights are gained into how the social ties were used to secure the economic benefits. 
through social contacts, has been largely neglected in network studies. ${ }^{8}$ By specifying the precise content of links, one can avoid some common pitfalls of social capital analyses: First, by not conflating links with myriad contents into one 'social tie' allows for the fact that people may source different types of resources from different contacts. For example, one may seek emotional succour from one's close friends but problem-solving advice is probably best-sourced from an acquaintance who has faced a similar problem earlier. Ignoring the link content in this example forces one to conflate emotional support with problem-solving knowledge and confuse the two otherwise (partially or completely) separate networks. In contrast, testing the overlap between different networks (that transfer different resources) allows one to reflect on the nature of embeddedness of one type of exchange in another. ${ }^{9}$ Second, wellaccepted results on social capital due to a strategic position in social networks may be erroneous if the links transferred a different resource than the one on which the results were based. For example, it has been shown that the effect of holding a strategic brokerage position in an intra-organizational network may be downward job mobility if the links transfer social support and upward mobility if the links transfer information (Podolny and Baron 1997). Third, observing the link content can permit one to differentiate social capital as a boon from social capital as a bane. Membership of dense social groups can have its negative impacts such as social control. This downside of social capital is overlooked if the social is equated to conviviality, solidarity, and support (more on this aspect in the following).

By focussing on the structural feature of social capital (based on an individual's position in a social network), sociologists have observed two main types of social capital: first, bonding social capital is available to individuals due to membership in a social group or clique that is densely connected. Here individuals identify with their social group and do not act according to narrowly defined self-interest but rather identity-related interests defined by the group's norms and values. ${ }^{10}$ Bonding social

8 Exceptions include Flap and Völker's (2000) distinction between strategic ties (instrumental for doing one's job) and solidarity ties (the "social" cooperation and trust at the workplace); Podolny and Baron (1997) on ties transferring information or social support; work on strong and weak ties by Granovetter (1973), Bian (1997) and others. The distinction between weak and strong ties is also often reduced to network-structural terms: the former are instrumental in spanning structural holes whereas the latter form the basis of social capital through closure (Özman 2005). In addition, see Stokman's (2004) attempt to develop a heuristic for combined analysis of network structure and content based on social exchange and group theories. Detailed work on tie content in the Netherlands was done by van der Gaag and Snijders (2005) on resource generator approach (a combination of name generator and position generator approaches) in the Survey on the social networks of the Dutch, but network structure was missing from their analysis.

9 Interest in the embeddedness of economic exchange in social relations, first studied by Polanyi (1944), was revived by Granovetter (1985) and has been a popular subject among economists and sociologists since the latter's work.

${ }^{10}$ An individual's choice to join a group (or remain in one) may be a function of who they want to be, or in a more static understanding, what they are (Kirman and Teschl 2006). The latter static understanding is still relevant in cases such as caste-based identity where 'what an individual is' gets 
capital benefits group members through trust or conviviality in socio-economic exchanges. However, bonding social capital may have a downside that an individual may be pressured to act in conformance to the standards of her own group only. This may limit an individual's capacity, for example, to access new information and knowledge from outside her group (note the conflation of different link contents here without any change in the overall significance of bonding social capital: this underlines the primacy of network structure in economic sociology). Possessing the second type of bridging social capital, generally held by brokers or people who connect two otherwise (horizontally) separate social groups, allows an individual to overcome the constraints and pressures imposed by bonding social capital. ${ }^{11} \mathrm{~A}$ variant of the bridging social capital which allows individuals to bridge vertical divides such as those due to inter-group hierarchies may be distinguished as well. Szreter (2002) terms this hierarchy-bridging type as linking social capital. The criticism of the neglect of tie content outlined in previous passages applies to the structural notions of bonding and bridging social capital: A map of the links in a network (the network structure) alone is insufficient to perform an in-depth analysis of social capital, as defined by Bourdieu, and may yield erroneous results. A detailed analysis to uncover both positive and negative aspects of social capital requires some information on the nature of the local intra-group politics and the inter-group divide: the power inherent in the interactions between any two individuals or groups. Performing such an analysis of the benefits received or freedoms curtailed due to social capital forces one to bring the content of links in a network back into the picture.

In part II of this thesis, I argue that it is beneficial to view a local system of innovation (or a community) as a set of social networks embedded in a broader society/economy, but this view must be made complete by a precise specification of the content of links in the social networks. For example, do the links transfer knowledge, goods, or socio-political support, or some or all of the three? Coherence in this system of innovation then implies the possibility of sustained decentralised action by the involved actors, through the mobilisation of different forms of individually-held social capital, in order to promote a desired technology in the knowledge, goods, and socio-political domains separately and jointly with overlaps in more than one domain.

For example, in the knowledge domain coherence as sustainability implies that the desired technology continues to be used and developed after the withdrawal of the donors and experts who initiated the project. For achieving this, the control and management of the technology should move into the hands of the people who use (or intend to use) the technology as a component in their production process. In the case

defined at birth and cannot be changed over her lifetime. Of course, there is life (and identity) beyond caste even in rural India and an individual may be a member of many parallel social groups due to multiple characteristics or qualities of her personality.

11 The terms bonding and bridging social capital are taken from Putnam (2000). He used these labels for grouping two streams of literature in economic sociology, focussing on cohesion and brokerage respectively (see Burt 2000; and Cowan and Jonard 2006 for brief overviews). 
of NPM in the village, I argue that this can only happen if farmers are able to mobilise adequate social capital for knowledge relevant for widespread use and further development of NPM. This form of social capital for knowledge among the farmers can be observed structurally in the form of their position in the knowledge network. If (some of) the farmers for example cooperate with each other to constitute a formidable core structure, they can provide the necessary dynamic sustainability to the local system of innovation in the knowledge domain.

But coherence in the knowledge domain alone is not sufficient to ensure the dynamic sustainability of a local system: corresponding coherence may be necessary in the business and socio-political domains individually and jointly where the different domains overlap. For the latter, we must pay attention to the link-content component of social capital, beyond knowledge into the other (non-knowledge) types of linkcontents. While some of the knowledge links of the village may get buttressed by social support, trust, conviviality, or economic exchange, some others may not. A different form of social capital for knowledge then enters the picture, manifesting as trust or conviviality. This social capital will be a function of the number of social ties transmitting trust or conviviality which also transfer knowledge. A coherent combination of the social network transmitting socio-political support (or financial credit for purchasing farm-inputs for example) and the knowledge network may well be the secret formula for long-term sustainability of community-driven initiatives. However, such positive reinforcements between different domains of social life, which make an entire socio-technical system dynamically sustainable, may lock us in to this system for better or for worse, making a transition to a newer one very difficult if not impossible.

\section{I.I Outline of the thesis}

The thesis is divided into two parts. Part I, composed of two chapters, deals with issues of technological interdependence and system evolution and is more theoretically oriented. Part II, based on empirical data on networks of an Indian village, is focussed on social capital. Results from part I are used to analyse the data in part II. In the following, I provide a brief summary of each chapter in the two parts.

Chapter 2 builds a theoretical background for part I through a literature review of the mechanisms and structure of technological interdependence. Technological interdependence is viewed as a result of the flows of knowledge and goods among actors. Four mechanisms of technological interdependence are discussed, namely technology succession and competition; horizontal interdependence in complex product and technology systems; generic technologies; and vertical relationships in a production chain. Subsequently, temporal aspects of technological interdependence are discussed and the gradual phases in evolution are differentiated from technological discontinuities. During the gradual phases, the set of interdependencies of a technology remains largely the same whereas a discontinuity brings about a disruption of this set. The final section of the chapter lays the foundations for a simulation model 
developed in Chapter 3. A literature review on the building blocks of the model, coreperiphery structure and the circular process (a cycle in graph-theoretic terms), is presented.

Chapter 3 develops a simulation model of knowledge creation and accumulation. Here I present one interpretation of an abstract simulation model (other interpretations and applications of the model are possible and have been attempted in the literature). Building on a review of models of technological evolution, the chapter provides a description of the model, followed by a discussion of the results and some simulation experiments. The results show three phases in the evolution of a technological system beginning with an experimental phase when new combinations are tried out. This ends with the emergence of a cyclic core which ushers in the coherent era of knowledge accumulation based on one of many possible combinations and finally, the third stable phase after a system of peripheral knowledge types around the core is completely built up. Stochastic appearance of a discontinuity in the form of a stronger competing core breaks the stability and brings in a new system built around the new dominant core. Simulation experiments are used to study outcomes of competition among cores of different strengths. Strength of a (cyclic) core is a function of the density of internal links within the cyclic formation, two competing cores' positions in the network relative to each other, and the flow of fresh ideas to a core through incoming links from peripheral components.

Part II begins with Chapter 4 where I introduce the community studied - a village in Andhra Pradesh, India which received widespread media attention across India for its adoption of pesticide-free farming (NPM) with the help of some NGO's. I identify three aspects of the village's successful experience with NPM reported in the media, namely the village-wide adoption and development of NPM; the repayment of debts by all farmers and freedom from dependence on the local farm-input dealers; and finally the adoption of a pesticide-free resolution by the village council.

In Chapter 5, I discuss the methodology used for collecting network data in and around the village. The nature of the required data necessitated the use of a mixed methodology: a personal interview-based survey with heads of all households in the village coupled with participant observation and data sourcing from NGO representatives. The focus of the data collection effort was both on networks within the village and on networks of the village to outside actors. The latter are important as a number of economic transactions of the villagers occur with people residing outside the village. Network data was collected for three main types of networks: knowledge flows for problem-solving in pest control, business networks of farmers for procurement of farm-inputs and credit, and the social network of the village focussing on close friendship and kinship ties. In addition, data on innovative performance of farmers was collected.

Chapter 6 focuses on the knowledge network and the innovative performance of farmers in the new technology, NPM. The innovative performance is measured using data on the extent of adoption and experimentation by farmers, collected with the 
survey. The farmers' innovative performance is then connected to the structure of the knowledge network which is analysed using the concepts of core and periphery, circular process, and core-strength (dominant core) developed in part I.

In Chapter 7, I discuss the 'success' of farmers in repaying their debts from the higher profits gained as a result of using the cheaper NPM instead of expensive pesticides, and the freedom from dependence on the local farm-input dealers who traditionally provided credit, sold farm-inputs and bought the farmers' crops. The success is evaluated and then analysed using data on the business network of the village. The structure of the business network casts doubts on the success of NPMdriven liberation of the farmers.

The socio-political activities of the villagers that led to the nationwide media publicity for the village are discussed through the lens of its social network in Chapter 8. Building on a brief literature review on social anthropology of multi-caste Indian villages and power in communities, a group of powerful leaders and brokers in the village is identified using centrality and brokerage measures from social network analysis. The structure of connections among these influential people is then analysed to determine if a circular process, and thus a dominant core, exists between them. This core group of people in the village carried out much of the liaison work with the media and the NGOs. These brokerage activities coupled with their influence among the villagers allowed the core group of people to project a unified face of success to the outside world for an extended period of time.

Chapter 9 studies the interpenetration of the three networks studied separately in the previous chapters. In particular, the focus is on the embeddedness of the knowledge network (studied in Chapter 6) in the social network of close friendships and kinship (analysed in Chapter 8). This social embeddedness of knowledge sharing is considered an essential aspect of local innovation systems on the one hand, and communities of practice on the other. I find limited evidence for the social embeddedness of knowledge flows. The analysis determines whether this embeddedness is found in the networks of the village and the networks within the village.

A final Chapter 10 summarizes and provides conclusions for the thesis as a whole. 


\section{Part I \\ Technological interdependence and evolving networks}




\section{Technological interdependence and knowledge flows}

The focus of the present chapter is on the interdependence between different types of technological knowledge (the knowledge dimension of technology). Here rather than analysing the characteristics of actors involved in the generation and use of knowledge, ${ }^{1}$ I study the structure of 'anonymous' technological knowledge or the systemic embeddedness of knowledge types in a larger technological system. Reasons for such an approach were discussed in chapter $1(\$ 1.1)$. They relate to the relationship between this structure and social networks (of knowledge flows; and to identify patterns in the evolution of technological knowledge.

This chapter is structured as follows: first I explore four different mechanisms of technological interdependence, largely in terms of knowledge flows. ${ }^{2}$ I show how these mechanisms make technological interdependence a widespread phenomenon in industrial and industrialising economies. I use existing case studies in the history of technology for finding various examples of technological interdependence. In a second section of this chapter, I discuss some temporal aspects of interdependence between different technologies. In particular, 1 look at two related phenomena: first, persistence of interaction between two or more technologies until a technological discontinuity breaks the interaction and ushers in a new 'era'; and second, a change in the degree or intensity of technological interdependence over time. In a third and final section, I focus on the structure of flow of goods and knowledge in an economy, starting with an overview of the core-periphery structures in systems and networks, as discussed by economic historians, management scholars, and economists. This is followed by a brief review of the idea of circular flow of goods in economic theory. I end the chapter by assembling theoretical and empirical evidence highlighting the importance of the circular process in knowledge-flows for an economy.

\section{I Mechanisms of technological interdependence}

It is illustrative to view technological interdependence in two mutually exclusive terms: substitution and complementary. Two interdependent technologies may either substitute each other, as in cases of technology succession; or they may be complementary to each other, as among the various components that go into assembling an automobile or the different phases in an automobile's production

\footnotetext{
${ }^{1}$ Most of the analysis in the present chapter is agnostic about the nature of actors. In different segments of the discussion below, I use the term actor to imply an individual; a sub-unit of a firm; a firm; or an industry.

2 The flow of knowledge in an economy cannot be completely disentangled from the flow of other dimensions of technology, such as the flow of goods and skilled personnel. In this chapter, I discuss some aspects of the flow of goods to complement knowledge flows wherever necessary. But a discussion on the movement of skilled personnel across different firms and industries is out of the scope of this thesis.
} 
process. Complementary technological interdependence may be sub-divided into two categories: vertical and horizontal. Such technological interdependence may arise due to horizontal and vertical relationships between industrial actors. A clear exposition of these relationships can be found in studies on industrial organization and organizational ecology (see for example, Richardson 1972; 1995; Astley 1985; Tirole 1988; Barnett 1990).

In the present context of technological interdependence, for example in a technological system or a complex multi-component artefact, I adopt a hierarchical conceptualisation of a system. Here a system is "composed of interrelated subsystems, each of the latter being in turn hierarchic in structure until we reach some lowest level of elementary subsystem" (Simon 1981: 196). If a technological artefact is treated as a system, its basic components are the elementary subsystems. All basic components, and subsystems, together with their linkages determine the 'architecture' of an artefact at the overall system level (Henderson and Clark 1990). Similarly, architecture of a multi-technology system, which may include interconnected sets of production processes, is determined by the structure of interconnections between its subsystems. Interactions between the subsystems can be horizontal and vertical. These two types of interactions may be illustrated through a visualization of a simple hierarchical system shown in Figure 2.1. Interactions between artefacts at the same level, such as those between two first level subsystems, that combine to form a second level subsystem, are regarded as horizontal interactions. On the other hand, interactions across different levels are vertical interactions such as the interactions between the basic components and the first level subsystem formed by them.

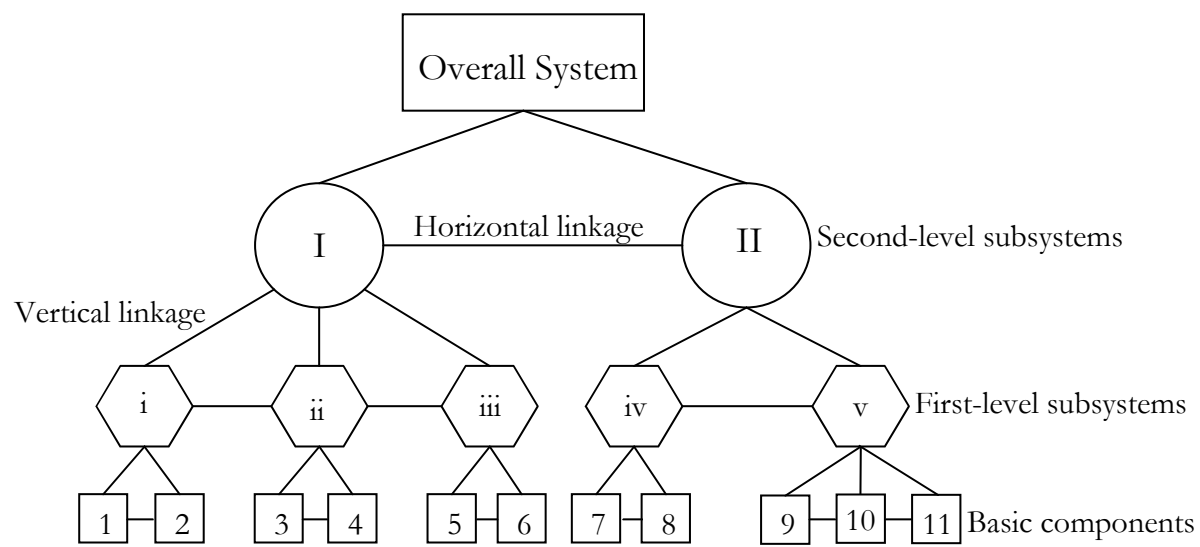

Figure 2.1: A simple idealized hierarchical system

A second classification of types of technological interactions is given by direct and indirect interactions. The horizontal and vertical links labelled in Figure 2.1 are direct interactions. Components interact with each other directly if they are part of the same subsystem or indirectly if they constitute separate subsystems. Further, two components 
or subsystems interact directly if the distance between them is only one linkage. For example as shown in Figure 2.1, horizontal-direct interaction exists between components 1 and 2, and vertical-direct interaction exists between component 1 and first-level subsystem i. If the distance between two components or subsystems is two or more linkages, the interaction between them is indirect. In Figure 2.1, borizontal-indirect interaction exists between first-level subsystems $i$ and iii, and vertical-indirect interaction between the basic components and any of the second-level subsystems. In general, the strength of direct interactions, or the degree of direct technological interdependence, will be greater than the indirect ones. Finally, the impact of interactions between two technologies at the overall system level may reverberate throughout the two systems affecting all components, whereas the impact of interactions between two basiccomponents may not be widely felt in a system.

Technological interdependence comes to the fore due to the social division of labour involved in the production and use of goods. A wide variety of actors who may be distributed across one or more industries generally provide technological inputs (goods, knowledge, services) in the production process of a good. This good may eventually be used as a consumer article or as an intermediate good for making other goods. In the case of a multi-component assembled good, the network of these actors may be visualized as Figure 2.1 if each actor manufactures a different subsystem. Then each node in the Figure represents an actor. In such a network, directed links run from bottom to top: actors who manufacture the elementary subsystems, or basic components, at the bottom supplying to the next tier of actors who make the first-level subsystem and so on. Once again note that the actors in this network may be distinct firms, or sub-units of a vertically (or horizontally) integrated firm, or individuals. Further in such a scheme, the vertical linkages between actors can be divided into two distinct types: backward and forward linkages. ${ }^{3}$ For example, for the actor manufacturing and supplying the first-level subsystem i, (direct) backward linkages lead to the actors making basic components 1 and 2, whereas the forward linkages connect to the actors responsible for manufacturing the second-level subsystem I and the overall system. Below I discuss four mechanisms of technological interdependence which are related to vertical and horizontal relationships in a technological system, such as the ones shown in Figure 2.1.4 It should be mentioned however that the

3 The terms backward and forward linkage are used in studies on economic development following Hirschman $(1958 ;$ 1992). He studied the stimuli that forward and backward linkages provide to investment flows in industrialisation. According to him (1992: 58), "The linkage dynamic made it possible to visualize the industrialization process in terms of an input-output matrix, most of whose cells would be empty to start with, but would progressively fill up in large part because of backward and forward linkage effects." Here the similarity to Leontief's input-output tables is obvious.

${ }^{4}$ Here I am referring to vertical and horizontal relationships between diverse actors such as firms, sub-units of firms, and industries. In turn, one may view horizontal and vertical technological interdependence as mechanisms that shape, and get influenced by, corresponding relationships among the actors, such as strategic alliances between firms or supplier-buyer relationships between industries. 
distinctions between the four mechanisms underlying technological interdependence may not always be mutually exclusive.

At the outset, it is important to specify the relationship between knowledge relatedness, a popular term in recent literature in economics of technical change, and technological interdependence. In a paper on knowledge relatedness and the technology diversification behaviour of firms, Breschi et al. (2003) explicate three aspects of relatedness:

a) knowledge proximity: Breschi et al. (2003) use this term to refer to the cognitive distance from the present technology of a firm to a new technology the firm starts to work with. This aspect of knowledge relatedness is similar to the interdependence between the new and the old substitutable technologies in a technology succession (as discussed further in $\$ 2.1 .1$ below).

b) knowledge commonality: An example of this type of interdependence may be found by comparing two very different fields of engineering, namely, chemical and aeronautical engineering. The knowledge of fluid mechanics is essential to both these disciplines. For example, aeronautical engineers use the knowledge of fluid mechanics to design better airfoils and to study the aerodynamics of aircrafts, whereas chemical engineers use similar form of knowledge to study reacting flows inside a chemical reactor. The aircraft and chemical reactor technologies are significantly different from each other, but a part of the knowledge content underlying these two technologies is common, resulting in a case of technological interdependence. ${ }^{5}$

c) knowledge complementarity: Complementarity between two technologies arises when one needs one technology to produce, or use, the other. Details on this aspect of knowledge relatedness, through forward and backward linkages in industrial production and knowledge creation, are discussed in \$2.1.3 and \$2.1.4.

\subsubsection{Technology succession and interdependence}

Technology succession implies the substitution of old forms of technology by distinct newer forms. In such cases, knowledge relatedness commonly arises between two substitutable technologies; and the two technologies compete with each other in the economy for some span of time. ${ }^{6}$ As an example of succession, consider the replacement of the handloom by the power-loom. A comparison between these two technologies shows that nature of the handloom heavily influenced the development

5 This type of interdependence due to similarities in knowledge bases may occur through the movement of skilled personnel from one field of technology to another. In addition, such interdependence may exist at the cognitive level of a technological practitioner, without the movement of or interaction between trained personnel across the two fields. Layton $(1978$, p. 72$)$ provides an example of knowledge interdependence at the cognitive level of a nineteenth-century inventor of the draft tube, "The emphasis upon creating a vacuum and working by atmospheric pressure [in the draft tube] is highly reminiscent of the operation of a condensing steam engine." This draft tube was used as a component of the improved reaction (water-) wheel. Here, one observes cognitive level interdependence between the steam engine and the reaction wheel.

${ }^{6}$ For classic studies on technology competition, see Arthur (1988; 1989) and David (1985). 
of the power-loom. In general, a new technology is often modelled, both in product and process characteristics, on the existing technology it is trying to replace. And only through multiple gradual modifications does a new technology, passing through different stages, becomes completely distinct from the older technology. Interpreting the gradual modifications from a systems perspective, a new technology typically has to 'fit' in with components of an old technology. For example, it took more than 30 years for diesel locomotives to completely replace their steam counterparts and during this time, the former had to run on the same track as the latter (Mansfield 1963). Similarly, the first nylon fibres had to be woven on standard textile machinery and pass through normal washing and dyeing processes used with natural fibres (specifically silk in this case: Hounshell and Smith 1988a). ${ }^{7}$

In cases of technology succession, the old technology generally co-exists with the new for some time. The old technology may be improved after the introduction of a new competing technology due to the so-called 'sailing ship effect' (Rosenberg 1976; Musson 1976; Pistorius and Utterback 1997). The improvement of the old technology is often influenced by the form of the new technology. Therefore, in many cases of technology succession, there is a two-way relationship between the new and the old technologies.

\subsubsection{Horizontal interdependence in complex products and technology systems}

This mechanism of technological interdependence is related to the horizontal pressures and complementarities created inside a complex multi-component artefact or a technology system. Subsystems at the same level are horizontally complementary to each other (the second-level subsystems I and II in Figure 2.1). For example, such horizontal interdependence exists between the engine and the gearbox subsystems of an automobile, which must be designed to be compatible with each other. If knowledge underlying each component of a complex artefact is different, then the knowledge base of an actor manufacturing a component will be distinct from others. For example, in order to manufacture the components of computer-numerically controlled (CNC) machine tool, some actors may need knowledge of computer software and electronics, whereas others must know the dynamics of machines. In such cases, the different types of knowledge may be viewed as horizontally complementary to each other. 8 These interacting 'knowledge components' may lead to

\footnotetext{
7 If the same process is viewed through the lens of an old technology, only a small component of an old technology is generally replaced at a time in its gradual transformation into a new technology.

8 Horizontal cooperation between actors, in technology alliances and networks, may occur due to horizontal complementarity. Such networks may be inter-firm or intra-firm. Inter-firm networks allow firms to access horizontally-complementary knowledge types (see Orsenigo et al. 2001 for an analysis in the pharmaceutical industry). Similarly, intra-firm networks between different sub-units of a multi-divisional firm may consist of horizontally-complementary relationships in addition to the usual vertical connections.
} 
the development of whole new fields of engineering such as mechatronics: a phenomenon often called 'technology fusion' (see for example, Kodama 1991; 1992).9

In a more dynamic framework, a change in one component of a multi-component artefact may produce a cascade of interdependent adaptations among the other components of the system. These horizontal 'technical imbalances' act as important factors in directing further developmental activity (Rosenberg 1976). This phenomenon is illustrated by the following example from Rosenberg (1976, p. 114) about the introduction of high-speed steel for making cutting-tools in machine tools: high-speed steel made it

"possible to remove metal at dramatically higher speeds and also by taking heavier cuts in the metal...It turned out, however, that it was impossible to take advantage of higher cutting speeds with machine tools designed for the older carbon steel cutting tools because they could not withstand the stresses or strains or provide sufficiently high speeds in the other components of the machine tool. As a result, the availability of high-speed steel for the cutting tool quickly generated a complete redesign in machine tool components - the structural, transmission and control elements."

In this example, the adaptations required in the different parts of the machine tools involved knowledge of three diverse fields of material science, power systems, and mechanical technologies.

Extending the above discussion to a multi-technology system, Hughes' (1983; 1987) large technology systems approach may be used to assess horizontal interdependence among system components. Components of a technology system include manufacturing firms, utility companies, investment banks, scientific components like books, journals, universities as teaching and research institutions, legislative and regulatory norms, natural resources, and physical artefacts (Hughes 1983). These disparate components interact with each other to perform the system functions. People as inventors, engineers, managers, financiers and workers are the active components who design the functions of a system and provide feedback between the goals and performance of a system. According to Hughes (1983), systems tend towards a hierarchical structure over time, such as the simple system shown in Figure 2.1 and the core-periphery structure between system-components discussed in $\$ 2.3 .1$ below.

If one views manufacturing firms, utility companies, and investment banks as subsystems at the same level in the hierarchical structure of a system, the nature of horizontal interdependence between them is multi-faceted. For example, knowledge from disparate fields such as accounting and managerial strategy, in addition to the different types of technological knowledge, may be shared between these system components. Generally, all exchanges in terms of inputs and outputs within a technological system occur through interfaces between its different subsystems and

9 Here, vertical interdependence exists between mechatronics and its constituent knowledge types such as electronics. 
components. ${ }^{10}$ This refers to the flow of goods and knowledge through horizontal and vertical linkages between system-components. I discuss some possible structures of these flows in a system in $\$ 2.3$ of this chapter. There I show that circular structures of flow, of goods and knowledge, possess certain self-sustaining qualities that make the components of such structures the core-components in a system.

\subsubsection{Generic technologies}

The third mechanism is related to the generic technologies required for production in a modern economy, i.e. means of communication and transportation, and provision of energy sources. Some authors have termed these generic technologies as generalpurpose technologies, GPTs (see for example, David 1990; Bresnahan and Tratjenberg 1995). In general, these technologies have horizontal and vertical complementarities with a broad range of technologies in an economy.

Generic technologies, such as communication, transportation and energy technologies, provide 'appropriate conditions' required for smooth functioning of industrial production spread across different firms and industries. Marx (1976 [1867]) considered these technologies as essential for success of the industrial revolution in England. In the two centuries since the start of that industrial revolution, generic technologies have undergone tremendous revolutions themselves; and their importance in present industrialised economies is probably greater. Recently several authors have underlined the growth-generating importance of the new generic technologies, referring to them as general-purpose technologies, GPTs (see for example, David 1990; and the different articles in Helpman 1998).

GPTs such as semiconductors and automobiles are ubiquitous in the present times, and their interdependence with other economic and technological activities is frequently discussed in the literature (see for example, David 1990; Bresnahan and Tratjenberg 1995; Helpman 1998; David and Wright 1999; Brynjolfsson and Hitt 2000). The input-side vertical (backward) linkages of the automobile manufacturers include those to the manufacturers of the engine, the transmission components such as the gearbox or axles, HVAC (heating, ventilation and air-conditioning) equipment, and steel fabrication. On the output side, the automobile is used to support and perform a wide variety of techno-economic activities from passenger-transportation in cars and buses to specialised vehicles for transporting perishable items such as food products, and so on. The extensive uses of transportation and communications equipment, and the resulting large set of forward linkages of these technologies, has great significance for learning in an economy. Benefits from improvements in a transportation or communication technology, in response to needs of an actor linked through a forward linkage, are frequently passed through other similar forward linkages to a broad range

${ }^{10}$ In addition, inputs and outputs occur between a system and its external environment. Most systems are open systems that possess an interacting environment, however technology systems tend to incorporate elements of their environment over time which might lead to an ideal closed system (Hughes 1983). 
of actors in an economy. Related importance of forward linkages in inducing investments, for example through initial investments in key technologies such as capital goods, in economic development was pointed out by Hirschman (1958).

Horizontal interdependencies of transport and communication technologies are probably less widespread than their (output-side) vertical linkages, but examples can be found in the history of technology. A horizontal interdependence between the bicycle and the automobile comes to the fore by considering them as two modes of individual transportation available to buyers at the turn of the $20^{\text {th }}$ century; the bicycle being the older technology. According to Hounshell (1984: 214),

"Both the bicycle and the bicycle production technology provided the basis for the age of the automobile in America."

He adds,

"When sufficiently large, the automobile industry also demonstrated the importance of finishing, assembly, materials flow, quality control and testing, which...became critical for a few bicycle makers such as the Pope Manufacturing Company. Henry Ford and the production experts at the Ford Motor Company especially recognized the problems that had arisen as a result of high-volume output, capitalized on some of the solutions offered by the bicycle industry (such as presswork and electric resistance welding),..." (Hounshell 1984: 214-215).

A similar horizontal interdependence can be observed between guns and the first internal combustion engines (a core-component of an automobile). This horizontal interdependence in terms of knowledge flows is illustrated by Mumford (1934: 88),

"the gun was..., mechanically speaking, a one cylinder internal combustion engine: the first form of the modern gasoline engine, and some of the early experiments in using explosive mixtures in motors sought to employ powder rather than a liquid fuel."

In addition to the transportation and communication technologies, energy systems act as another general condition required for the smooth functioning of various production processes in an economy. Energy systems capable of providing sufficient motive power to run integrated systems of large machines, chemical reactors, railways, computers, satellites, and all other forms of equipment, are essential for production. Therefore, just like communication and transport technologies, energy systems have extensive output-side vertical linkages. Consider the case of steam power. One of the most important output-side linkages of steam power in nineteenth century England was the set of cotton textile technologies. The 'steam revolution was predominantly in cotton' in the first half of the nineteenth century (Musson 1976; von Tunzelmann 1978). In the second half of the nineteenth century, massive growth in steam power was observed in England and other industrialized areas of the world, which saw a rapid expansion in its output side linkages (Musson, 1976, Bruland, 1998). Technologies such as coal-mining, primary iron-production technology, water pumping, brewing, grinding and milling may be regarded as significant forward linkages of the steam engine during that period (Musson, 1976). 
Important input-side or backward linkages of steam power (boiler, engine) include steel and the manufacturing technologies. In a study on the famous boiler manufacturing firm, 'The Babcock and Wilcox Company', Bruland (1998) shows that the development of a 'non-explosive' boiler was constrained by the firm's lack of capability in precision manufacturing. This constraint was removed through an arrangement with the Singer Sewing Machine Company for supply of the crucial 'header' component. Singer had gained precision manufacturing expertise through the manufacture of sewing machines. ${ }^{11}$ Similarly, earlier in the development trajectory of the steam engine Wilkinson's improved boring mill was central to the development of Watt's steam engine, as illustrated by Rosenberg (1976: 112),

"... James Watt's protracted search for a cylinder bored to a minimum degree of accuracy in its diameter. The required degree of precision was eventually attained with the use of John Wilkinson's boring mill, and the commercial practicability of the steam engine really dates from the use of this mill in preparing cylinders."

\subsubsection{Vertical relationships}

The last mechanism of technological interdependence is due to vertical relationships between different actors involved in the production of a good, in terms of complementary phases of a production process. Each dyad in this network represents a user-producer relationship. As a whole, this network of actors may be referred to as the production chain of input (or backward) and output (or forward) linkages.

Direct technological interdependence in the process of production begins with the flow of goods and knowledge between a seller and a buyer, or a producer and a user. These dyadic user-producer interactions are embedded in a network of actors engaged in producing a good at different stages of the production process. This network of vertically-linked actors may be referred to as a 'production chain'. A production chain consists of input-side linkages, both intra- and inter-industry, that enter the production process of a finished good; a set of output-side or forward linkages which determines the ways in which a given good is used in the economy, for example, the good may be used by other industries to produce other products or, as a consumer end-product. A difficult issue in studies of vertical relationships within and between industries is defining the boundary of the system under study, which must to some extent be arbitrarily drawn. For example, Williams (2003: 344) takes the following interconnected industrial sectors to represent the production chain of silicon: "quartz, charcoal, coal, "crude" silicon, chlorosilanes, polysilicon, silicon wafers, semiconductors, solar cells

\footnotetext{
${ }^{11}$ Here a horizontal interdependence between the sewing machine production technology and steam engine (more accurately, the steam boiler) is obvious. Secondly, if the precision manufacturing machine tools are technology in question, its forward linkages include the sewing machine and the steam boiler. Here improvements due to interdependence with one of the forward linkages is passed to other forward linkages. As mentioned earlier, this is an important characteristic of the GPTs.
} 
and optical fiber."12 If polysilicon is considered the nodal product in question, its backward linkages include quartz, charcoal, coal, crude silicon and chlorosilanes, whereas the remaining are its forward linkages.

Kemp et al. (2000) use the term production chain in a broad systems approach. They include cultural constraints, legal frameworks, regulations, infrastructure and other institutions which affect the linkages and their nodes in a production chain. In the present discussion, I adopt a more restricted interpretation, using the term 'production chain' to refer to the network of inter- and intra-industry linkages that govern the exchange of goods and knowledge on input and output sides of a specific product. Wallerstein (1983) uses the term 'commodity chain' to refer to a similar concept. According to him, a market-place where a product is sold to an endconsumer by a producer accounted for only a small percentage of economic transactions historically,

"Most transactions have involved exchange between two intermediate producers located on a long commodity chain. The purchaser was purchasing an 'input' for his production process. The seller was selling a 'semi-finished product', semi-finished that is in terms of its ultimate use in direct individual consumption.” (Wallerstein 1983: 29).

The knowledge counterpart of the above good-exchange between a seller and a buyer may be viewed as a user-producer interaction among two vertically linked firms or sub-units of a vertically integrated firm. User-producer interactions allow knowledge exchange between interconnected firms, and thus are critical for learning in a system of innovation (see for example, Freeman 1987; Lundvall 1988; Lundvall 1992; Nelson 1993; Freeman 1995). The dyadic knowledge exchange is embedded in networks of relationships with other organisations such as supplier firms, universities, research centres, government departments, financial institutions and end-users. The new knowledge required for innovation is generally created through joint efforts of the above knowledge generating actors operating in an informal institutional setting and with the support of formal institutional actors. Together these organizations and institutions form a system of innovation (SI).

According to Lundvall (1988), the starting point of an innovation system is the set of firms and their linkages in a national system of production. ${ }^{13}$ Thus, bidirectional exchange of knowledge among vertically connected actors (user-producer interactions), the most basic form of circular flow, forms the crux of a SI. It is the first necessary condition for successful learning by interacting. Technological and organisational competence is built in this system through learning and generation of new knowledge which, together with appropriate institutionalization processes, signals the emergence of a national system of innovation (NSI). In general, one may consider the following

12 Williams (2003: 342) defines production chains as "networks of processes that follow industrial transformations from the extraction of raw materials to the manufacture of "finished" goods ..."

${ }^{13}$ Innovation systems may also be defined at other levels than the national system considered here. For regional innovation systems, see Cooke et al. (1998), and Doloreux and Parto (2005) for a review of this literature. On sectoral systems of innovation, see for example Breschi and Malerba (1997). 
organisational bodies as critical components of a NSI (Lundvall 1992; Lundvall et al. 2002),

- Firms including organisations such as consortia of firms or sub-units of firms such as industrial R\&D laboratories

- Universities as sources of trained personnel and basic science

- Government sponsored research centres as crucial catalysers of privatepublic interactions

- Investment banks and other supporting organisations in the financial sector such as venture capital firms

- Relevant regulatory bodies such as patent offices or national/local government departments

- End-users, including public procurement by the government

The efficiency of a NIS is history-contingent and depends on the competence of the above organisations in producing, transferring, and absorbing new knowledge, and adapting to a changing external environment. It also depends on the evolution of the structure and efficacy of inter-organizational links within the system as knowledge of diverse types is exchanged and accumulated.

\subsection{Temporal aspects of interdependence: gradualism and discontinuities}

I adopt a punctuated-equilibrium based view of technological evolution where a gradual process of knowledge creation and accumulation is disrupted by the 'arrival' of a discontinuity (cf. Levinthal 1998). ${ }^{14}$ I show that during the gradual phase, the set of interdependencies of a technology generally remains the same even as the interdependent technologies gradually develop. A discontinuity brings with it, partially or completely, a new set of interdependent technologies and inaugurates a new phase of systemic technological development. Consequently, the punctuated-equilibrium model of technological evolution applies to the evolution of technological interdependence as well. On the one hand, a set of interdependent technologies provides inertia to the set (or production chain) as a whole. On the other hand, arrival of a discontinuity leads to a possible exit from the inertia generated by existing technological interdependencies, through gradual piece-by-piece construction of a new set of interdependencies.

An important aspect of a technological discontinuity, and the disruption of set of interdependencies it ushers in, is the time taken by a new technology to replace the

14 See Gersick (1991) for a general overview of punctuated equilibrium from six disciplines; Gould and Elderedge (1993) for an overview of punctuated equilibria in biological evolution; Romanelli and Tushman (1994) on organizational change; and studies in Mowery and Nelson (1999) provide evidence for punctuated equilibrium in the evolution of industry structure. Also see Kuhn (1970) for a discussion on 'normal' and 'paradigmatic' changes in the history of science and Dosi (1982) for a similar take on technological change; Tushman and Anderson (1986), and Anderson and Tushman (1990) on technological discontinuities. More details are presented in $\$ 2.2 .2$ and the next chapter. 
pre-discontinuity mature technology. For example, according to Musson (1976) and von Tunzelmann (1978), it took longer than a century, after Watt's first patent in 1769, for steam power to replace water-power as the dominant mode of motive power for industrial production. David (1990) points to a similar gradual emergence and growth of the electric power systems in his discussion on the so-called 'productivity paradox'. On the other hand in the case of nylon's synthetic fibre revolution, it took only a decade for nylon to gain prominence and widespread application. One may then ask: why does it take longer to build some technological systems than others? This important question may be easily brushed aside by pointing to differences in the size and complexity of different technological systems. However, one needs to break open the 'black box' of complexity to find plausible answers to this question, which has received only scant attention in the literature. I present a way to comprehend some aspects of this complexity through a core-periphery model of technological evolution with interdependence between multiple knowledge types in chapter 3.

In this section, using examples from history of technology, I demonstrate the persistence of a set of interdependent technologies during the gradual evolutionary phase between discontinuities. This is followed by some examples of disruption caused by technological discontinuities. However, based on a few historical examples recounted below, one cannot regard gradualism and punctuated equilibrium as generally applicable laws of technological development. The following discussion is merely an attempt to identify some widely observed patterns.

\subsubsection{Gradualism and persistent technological interdependence}

The development of steam engine is a neat illustration of gradualism in technological evolution. At the dawn of the nineteenth century in England, steam power, still in its infancy, was displacing hydraulic power very gradually. The development of the steam engine was a long protracted process spanning decades, involving incremental change and efforts of a large group of inventors. According to Usher (1954: 68),

"The history of the reciprocating steam engine involves at least five strategic inventions: the atmospheric engine of Newcomen; the low-pressure engine of Watt; the high-pressure engine of Trevithick and Evans; the steam locomotive of Hackworth and Robert Stephenson; the compound engines."

Throughout this period of incremental change in the steam engine, parallel developments can be observed in its interdependent technologies. In order to demonstrate this phenomenon of gradual reciprocal adaptations among interdependent technologies, I revisit the example of the steam engine and machine tools. The interdependence between James Watt's steam engine and Wilkinson's boring mill and other machine tools was instrumental in bringing about many improvements in the efficiency of the steam engine over the course of a century. These efficiency-improvements of the steam engine were made possible, in large part, due to better machining capabilities of the machine tools. In turn, demands from the steam 
engine developers led to many developments in the machine tools. This phenomenon of gradual changes was noted by Usher (1954: 359), beginning with a reference to Wilkinson's improved boring mill,

"His achievement made the steam engine immediately practical, though the early engines left much to be desired in accuracy of workmanship despite steady improvements in methods. The great balance beam was thus long retained, because it was not possible to make surfaces accurate enough to enable the engine builder to attach the crosshead to the crank. It was left to Maudslay to make this simplification in the machine [steam engine] after his improvement in machine tools" (emphasis added).

The use of words "steady improvement in methods" by Usher underlines the gradual nature of the development process of the steam engine, and that of the continually interdependent machine tools technology. The co-evolution of the steam engine and machine tools continued well into the nineteenth century, as illustrated by Musson (1976: 420),

"The average horse-power of all the Boulton and Watt engines built up to 1800 was only 15 h.p., and though the power and efficiency of steam engines were increased in the first half of the nineteenth century, with developments in machine-tools and boiler-making, this growth occurred very gradually..." (emphasis added).

In addition, machine tools possessed widespread interdependencies with many other technologies during the nineteenth century, such as firearms, the sewing machine, bicycle, typewriter and agricultural implements industries (Rosenberg 1976; Hounshell 1984). Many of these interdependencies continue to the present-day, although the different technologies have generally evolved from their original configurations of the $19^{\text {th }}$ century. This gradual evolution in machine tools' technology was noted by Rosenberg (1976). He points out that machine tools were significantly developed due to the demands by the sewing machine industry and the improvements suggested by the sewing machine makers. The resulting improvements in machine tools were also beneficial to the bicycle industry. ${ }^{15}$ Now if the sewing machine makers were to directly benefit from some developments in the bicycle industry, we arrive at a circular structure in the flow of knowledge between the three technologies. This circular structure in cooperative technological interdependence introduces a stronger element of inertia, than a linear structure of interdependence, into an existing technological system due to two interrelated factors. First, positive feedbacks among the constituent technologies of a circular process make it self-reinforcing and as a result, stable over time. Second, if a technology is integrated into a complex network of technologies, of which a circular process is probably the simplest example, its replacement by a new technology becomes difficult simply because of the resilience created by its rich network of interdependencies. Furthermore, a new replacement of a technology that forms an element in multiple partially-overlapping sets of

${ }^{15}$ Like the generic technologies discussed in $\$ 2.1 .3$, machine tools have a large set of output-side vertical interdependencies; results of learning in machine tools are useful in many industrial sectors. 
interdependent technologies, as GPTs do, may work for only one set of its interdependencies but not for the rest. This leads to the persistence of an existing (general-purpose) technology in the economy. ${ }^{16}$

The historical examples presented in the foregoing demonstrate the persistent nature of technological interdependence in a gradual co-evolutionary process of parallel adaptations among interdependent technologies. The set of technological interdependencies remained largely intact in gradual incremental technological evolution. This gradual evolution or co-evolution may however be interrupted by a technological discontinuity.

\subsubsection{Technological discontinuities}

A radical innovation leading to a technological discontinuity generally does not simply become a component of an existing technological system. A discontinuity initiates technological development along a new path, disrupting the existing pattern of systemic knowledge accumulation (Hughes 1987; Rosenberg 1982; Sahal 1981; Tushman and Anderson 1986). Synthetic fibres are a prime example of such a discontinuity which led to many small innovations and adaptations in interdependent technologies in the textile technological system. For example, the existing knowledge of textile spinning was rendered useless for spinning synthetic fibres: a new chemical process called melt-spinning had to be developed (Hounshell and Smith 1988a).

In Marx's (1976 [1867]) description of the textile industry at the start of the industrial revolution in England, the backward linkages of the ready-to-weave yarn were to cotton or some other natural product, and the spinning technologies. ${ }^{17}$ The forward linkages led to technologies that included machine weaving, and the chemical and mechanical technologies used in bleaching, printing, and dyeing. In the twentieth century, human-made fibres have largely supplanted natural fibres in textile production

16 Getting a new technology to fit in with all the technological systems it can be a part of, or alternately adapting the different technological systems to the new technology, takes an extended period of time (when it is possible). This may be a reason why new technologies often emerge in small niches where they are developed further to become more versatile, or have to wait for 'right' large-scale socio-technical changes, before getting more widely absorbed in the economy (Rip and Kemp 1998; Levinthal 1998; Geels 2002).

${ }^{17}$ Here spinning technologies include a series of specific operations. Wallace (1978) describes these operations in a cotton-spinning mill in 1820s south-eastern Pennsylvania where the technology was largely derived from England at this time. According to him, each of these operations "had its own location, its own machine, and its own operatives. After the bales were opened, the cotton was blended to produce a uniform staple length and was run through the picker room; then it was transferred to the carding engine; then to several preparatory spinning devices, including the double speeder, the drawing frame, the roving frame, and the stretcher; then either to the mule or to the throstle for final spinning of usable yarn; then, for some of the yarn, to the dyehouse; and finally to the warping machine or to the machine for spooling yarn on bobbins or cops specially prepared for insertion into shuttles" (p. 136). For the sake of tractability in the present discussion, we choose to encompass all these operations and machines, some of which may be considered as separate technologies in their own right, under the single heading of 'spinning technology'. 
and have led to dramatic changes in the backward and forward linkages of the readyto-weave yarn. ${ }^{18}$ Examples of human-made fibres are rayon, nylon, dacron polyester and orlon acrylic. Rayon is a cellulosic fibre, whereas the remainder are non-cellulosic or synthetic fibres (Hounshell and Smith 1988a). The nature of the discontinuity in the form of synthetic fibres is nicely summed up by Hounshell and Smith (1988a: 384),

"the synthetic fibers revolution entailed the rapid development of novel production machinery, and it wrought change in backward linkages, such as raw materials and intermediates, and in forward linkages, such as dyeing, finishing, and fabrication of apparel, carpeting, and entirely new structures that could not be made with natural fibers."

The first synthetic fibre was discovered by a scientist at Du Pont's research laboratory in 1930. This fibre was a polymeric substance that had to undergo half a decade of further development to become nylon (Hounshell and Smith 1988a). The first important step in the use of nylon fibres was that they had to be supplied in a form appropriate for the existing interdependent textile technologies. In turn, the existing textile technologies had to be adapted to the new nylon fibres. These two tasks were greatly facilitated and fast-tracked due to an early strategic decision by the Du Pont management: to restrict the initial applications of nylon in making replacements for women's silk hosiery. As a result of the initial focus on a well-defined market, technological adaptations were required in only a small subset of the interdependent textile technologies (Hounshell and Smith 1988a; 1988b). Moreover, the high market price of silk reduced the pressure of competition on a new material such as nylon that was the result of years of expensive science-based research and development. Also, initial experiments with nylon fibres revealed its similarity to silk in terms of strength and lustrousness.

Two backward linkages of natural yarn were central: the production of natural raw material and spinning. As compared to the same two technologies for natural fibres, the technologies used to make nylon fibres were mostly new. The nylon yarn for the stockings was made out of the nylon 6-6 synthetic polyamide that replaced the natural raw material, silk. The production of the nylon polyamide required the intermediate chemical compounds of adipic acid and hexamethylene diamine, which are produced by high-pressure catalytic methods. The natural silk fibres or filaments, on the other hand, are produced due to the secretion of a viscous fluid from some special glands of a silkworm (the main commercial silkworm is the larva of the Asian silkworm moth). Small bundles of these natural silk filaments are then twisted together by silk-throwers to make the silk-textile yarn. In contrast, spinning nylon turned out to be a drastically new chemical process that took a few years to develop. In 1933, scientists at Du Pont thought it was impossible to spin textile yarn out of synthetic fibres because of the properties of high melting point and low volubility required in the yarn (both of which lacked in the synthetic fibres they had at their disposal, Hounshell and Smith 1988b).

18 Human-made accounted for two-thirds of the total U.S. consumption of fibres in 1980 (Textile Organon, 1981, cited by Hounshell and Smith 1988a). 
By mid 1937 however, after tackling several obstacles in developing a commercially viable continuous spinning process, ${ }^{19} \mathrm{Du}$ Pont had found a solution to the spinning problem but had to perform the task in-house. It was not possible to spin nylon fibres with the existing technologies of the silk-throwers because nylon spinning turned out to be a sophisticated chemical process called melt spinning. A simplified description of melt-spinning is provided by Hounshell and Smith (1988a: 260),

"This process entailed melting the solid polymer to a honeylike liquid that would be driven under pressure through a number of very small holes in a metal plate. The extruded filaments would form solid fibres upon cooling."

Here it is important to note that, apart from polymer engineering, Du Pont's experience with other technologies like ammonia and rayon manufacturing proved essential for making nylon in a textile-suitable form (Hounshell and Smith 1988a). Thus the development and production of nylon 'synthetic' fibres led to drastic changes in the old set of backward linkages in the production of textile yarn.

Some changes were also observed in the forward technological interdependencies of yarn due to the use of nylon fibres. The first time that Du Pont sent its melt-spun nylon fibre for a knitting test at a silk-hosiery manufacturer, numerous problems were experienced with the new fibre in nearly every operation of hosiery manufacturing. "It did not come off the spools properly; it snagged on the knitting machines; and after dyeing it looked like a wrinkled mess..." (Hounshell and Smith 1988a: 266). More problems were encountered with other forward linkages in large-scale production with nylon fibres. Of these, a serious one related to the wrinkles produced in nylon stockings during dyeing and other finishing operations. This led to a modification of the dyeing technology to get rid of the wrinkles (Hounshell and Smith 1988a). Another formidable problem was experienced due to the lack of a natural coating or 'size' on nylon fibres (silk has a natural 'size' that protects the fibres during all textile-processing operations). Du Pont researchers solved this problem, on the eve of inauguration of the first full-scale nylon plant, by developing a new four-component chemical size for nylon (Hounshell and Smith 1988a).

In the foregoing, I discussed some of the changes that were required in different interdependent technologies due to the arrival of a technological discontinuity in the textile industry. In the example of nylon, the changes required on the output side of the production chain of yarn were not as drastic as on the input side, which was largely due to Du Pont management's decision to focus on the well-defined target market of silk stockings (Hounshell and Smith 1988a). Without the same advantage of a welldefined market, introduction of Du Pont's other synthetic fibres from roughly the same period, orlon and dacron, required more elaborate alterations on the output side. As a result, their success in the market was a much slower process than that of nylon (Hounshell and Smith 1988a).

19 See Hounshell and Smith (1988a: 259-267) for a detailed account of the development of the spinning technology for nylon. 
Similar to the example of textile technologies, other sets of technological interdependence were disrupted due to a discontinuity in one or more technologies of a production chain. Consider a broad example, the transport and communications technologies have undergone dramatic changes in the last two hundred years, with the computer and the automobile as two major discontinuities. In ways similar to the transition from natural yarn to synthetic yarn, new technologies were added to the set of interdependencies of transport and communication, to either replace or append the existing ones. Here the set of forward linkages of transport and communication technologies is much larger than that of the textile yarn. As a result, many sectors in an economy had to be adapted to the new transport and communication technologies. This process was essentially a gradual one and according to David (1990), a major reason behind the so-called 'productivity paradox'. It is difficult to trace all the changes brought about by a discontinuity in generic technologies such as transport, communications and energy technologies. For studies that have attempted to uncover the impact of a discontinuity in generic technologies, see Hughes (1983) and David (1990) on electric power systems; Lawless and Anderson (1996) and Anderson and Tushman (1990) on the case of the computer; on the automobile and its set of interdependencies, see Abernathy (1978) and Hounshell (1984); and on the replacement of water power by steam, see Musson (1976) and von Tunzelmann (1978).

In the above examples, the degree of disruption in the existing system-wide interdependencies, as a result of a technological discontinuity, naturally differs from case to case. Further, many technologies experience multiple discontinuities over the course of their evolutionary histories, for example, the personal computer industry has witnessed at least two: first in 1975 with a low-cost microprocessor chip based computer and then in 1981 a new architecture was released by IBM which became the 'original IBM PC' (Lawless and Anderson 1996). ${ }^{20}$

To summarize this section, I presented evidence in $\$ 2.2 .1$ to support the argument that the set of technological interdependencies often remains intact as the various interdependent technologies gradually evolve. This gradual evolution is broken by a technological discontinuity which disrupts the existing set of interdependencies. In \$2.2.2, I demonstrated the impact of a discontinuity by discussing an important invention originating in the chemical industry which profoundly affected the development of the textile industry: the synthetic fibre revolution. This technological discontinuity resulted in a (partial) disruption of the existing set of interdependent textile technologies. Below in the final section of this chapter, I discuss some other

${ }^{20}$ Further, Lawless and Anderson (1996) show that technological evolution in innovation-intensive industries, such as the personal computer industry, the phase between discontinuities is anything but a gradual phenomenon. They point out significant technological changes during the gradual phases and refer to those as 'generational technological change'. For the present purposes, unlike a technological discontinuity, generational technological change does not generally lead to disruption of the set of interdependencies, for example, it is completely compatible with its backward linkages (Lawless and Anderson 1996). 
cases of technological interdependence in a gradualist evolutionary setting, without discontinuities, in terms of the interdependence and knowledge flow between successive inventions. The interaction and evolution over a diverse set of technologies (or knowledge-types) then points toward a phenomenon of technological co-evolution.

\subsection{Structure of technological interdependence}

In this section, I build on the hierarchical structure of technological systems discussed at the start of $\$ 2.1$. In particular, I present an alternate way to conceptualise hierarchy in systems based on the concepts of core and periphery: cores are combinations of components which are more critical for the functioning of a system as compared to the periphery. This is followed by a brief review of the idea of 'circular flow' of goods in economic theory. Finally, I develop the concept of the circular process in the flow of knowledge among actors. I use historical examples to demonstrate a circular structure of flow between actors developing different incremental innovations. These incremental innovations may take place as acts of consolidation after the emergence of a discontinuity; or they may feed into each other to collectively act as a radical innovation. It is in the latter sense that the circular process becomes the core of a network of innovations.

\subsubsection{Hierarchy in systems: core and periphery}

Hierarchy between components of a system arises in two interrelated mutuallyreinforcing ways: first due to differential power of components in terms of a socioeconomic or technological characteristic such as market share, or knowledge complexity; second, hierarchy may manifest through the structure of connections between components or subsystems, for example, in terms of the number of linkages of a component with other components in a system. Here I explore core-periphery distinction among system-components along both these dimensions.

Characteristics of some subsystems may make them more significant than the others in a system: at the outset, such unequal significance arises due to the hierarchical relationship between subsystems, as shown in Figure 2.1. For example, small variations at the overall system level or at a high level subsystem, will have ramifications throughout the system, whereas minor changes at the basic component level may be less significant for the system as a whole (Clark, 1985). However, this form of reduction in significance with structural hierarchy may be only one way in which differential power or influence exerts itself in a system. In some other 'hierarchical systems' of interest, hierarchy may enter through a 'core-periphery' network structure. For example, components and subsystems that have a large number of linkages with other subsystems and components may be considered as core and the ones with fewer linkages as periphery (Murmann, 2001).

Core-periphery models

Before exploring the hierarchical and structural distinction between a core and a periphery in different strands of the literature, here I briefly discuss how a core- 
periphery arrangement may arise between the interacting components of a technological system. According to David (1990), productivity benefits from the use of electrical technologies had to wait the full development of an elaborate technological system with its core and periphery technologies. In addition, according to Hughes' (1983) large technological system framework, a nucleus of a system is of central importance. In purely technological terms, this nucleus may be considered as a set of component technologies that form the core of the system. In an electrical power system such a core for example may consist of the turbine, the dynamo, transformers and the electric motor. On the other hand, technologies such as the pylons, overhead and underground cables, and other fixtures used in electric transmission; and the various measurement technologies in the system, such as the ohmmeter, and the voltmeter may be considered as the periphery. In addition, periphery technologies are found in factories that use electricity, such as switches and rheostats (control systems), lighting systems, and transmission systems (for example, the belt and shaft equipment).

A key question here concerns the characteristics of components, or of their combinations, which make some of them core and others periphery. In the context of the present example, what factors make the dynamo and the transformers core, and the pylons and the transmission cables periphery? In terms of technological knowledge, the core-periphery distinction may be interpreted in two ways: First, using the notion of vertical linkages, backward linkages of the periphery almost always lead to the core. The periphery is then dependent on the core for its inputs in terms of knowledge and goods, but the reverse is not true (the core's backward linkages largely lead to other nodes within the core). Further, the few backward linkages from the core to the periphery do not make the core dependent upon the periphery for growth or stability. The core is largely a self-sustaining combination that may generate growth and stability for the system as a whole. Second, the knowledge base of periphery components depends upon the core, whereas the underlying knowledge of periphery components is not crucial for the core. For example, the knowledge of mechanical structures required for building pylons (periphery) may not be necessary to build a dynamo (core); but some basic knowledge of how electricity is generated, in terms of voltage ratings etc., is necessary for building effective pylons. It is obvious here that a small element of arbitrariness is involved in defining the core-periphery distinction empirically. Also, this distinction can be addressed from many different standpoints, ranging from influence and power to growth and innovativeness. The brief review of the literature in the following passages explores interpretations of the core-periphery distinction in different social-scientific areas: many authors contend that the coreperiphery distinction is a useful theoretical construct but difficult to establish empirically.

The core-periphery model has been interpreted in multiple ways in a variety of disciplines. A review of all the different interpretations is beyond the scope of this study. I discuss a few main conceptualisations to highlight two issues: first, the hierarchical and structural distinctions between core and periphery components of a 
system; and second, a very brief discussion on some possible core-periphery dynamics. I start with the core and periphery model developed by Wallerstein (1974) and some of its refinements attempted in later studies. Then briefly discuss Krugman's (1991; Fujita et al. 1999) use of the core-periphery model in economics. Lastly, I talk about the structure of connections within, and between, a core and a periphery focussing largely on the work of Borgatti and Everett (1999; Everett and Borgatti 1999) who formalised the core-periphery model using social network methods.

Wallerstein's (1974) model is based on the concept of a 'world-system' which is characterized by a single social division of labour across its different regions. A worldsystem or a 'world-economy' is divided into core-states, semi-peripheral areas, and peripheral areas. Core-states, as opposed to the peripheral areas, are industrialized regions possessing strong state machineries, complex economies with high capital investment per worker, and exercise political and cultural domination over other parts of a world-economy. In between the two opposites of core and periphery lie the semiperipheral areas that may have been core-states or peripheral areas in the past, making the transition from one to the other or trying (not) to. Interactions across core and periphery boundaries are dominated by members of the core; and internal interactions within non-core areas tend to be mediated by the core-states.

An important distinction between core-states and peripheral areas is defined by the nature of tasks performed by each. The task-based hierarchy in a core-periphery worldeconomy is illustrated by Wallerstein (1974: 350),

"The division of a world-economy involves a hierarchy of occupational tasks, in which tasks requiring higher levels of skill and greater capitalization are reserved for higherranking areas. Since a capitalist world-economy essentially rewards accumulated capital, including human capital, at a higher rate than "raw" labour power, the geographical maldistribution of these occupational skills involves a strong trend toward self-maintenance. The forces of the marketplace reinforce them rather than undermine them."

The "forces of the marketplace" provide the dynamic of this core-periphery model in which the "self-maintenance" of the core-periphery structure occurs due to advantages built inside the core-states; and the positive feedbacks operating between these advantages and the exchange with peripheral areas through international trade. As a whole, the web of positive feedbacks, both within the core and in its exchanges with the periphery, makes the core a self-sustaining phenomenon in the worldeconomy. It is not surprising then "the advantages of the core-states have not ceased to expand through the history of the modern world-system," (Wallerstein 1974:350). However, the composition of the core sector can change with time: a country may slip into the semi-periphery (or periphery) from the core at some point in time, and vice versa. But this circulation does not alter the fact that the core sector as a whole is always stronger than the peripheral areas.

A brief discussion on the conditions under which a core-state slips into the periphery, and vice versa, is apropos here. Wallerstein (1974) presents the case of 
northern Italy (Venice, Milan and Genoa) which slipped from the core sector to semiperiphery in the first half of the seventeenth century.

"From being one of the most advanced industrial areas in Europe in 1600, northern Italy became a depressed agricultural region by 1670." (Wallerstein 1974: 219).

He cites a host of reasons behind this fall including the lack of both political unity and large unitary market; plagues of 1630 and 1657; bad investments; loss of market in England and France due to the development of their own textile industries; loss of control of sea transport; increasing labour costs due to strong Italian guilds which made the industries non-competitive, and competition from other upcoming areas such as the northern Netherlands. An important point to note is that the reasons, behind northern Italy's transition from the core to the periphery, concern conditions internal to northern Italy i.e., conditions within the core; and other factors external to the core region, in particular the developments taking place in the periphery and semiperiphery areas, such as the Netherlands, that affected the network of trade in the world economy. Similar dynamics, resting on the structural conditions within a core and other developments originating in the periphery, drive the descent of core components into the periphery in a model on technological evolution presented in chapter 3.

Another important distinction between core and periphery within Wallerstein's world-system framework is provided by O’Hearn (1994) who uses innovation, rather than production, as the defining element of the "cutting edge between coreness and semi-peripherality within the global structure.” (p. 595). In this conceptualisation, semi-peripheral areas may be involved in advanced industrial production as long as innovation in that industry originates in one of the core-states. The same could be true for advanced industrial production and innovation in multiple sectors. I adopt a similar perspective on core-periphery in model presented in the next chapter, using innovation or innovativeness as a variable that distinguishes the core from peripheral elements of a system. ${ }^{21}$ The model uses a simple non-deterministic dynamic to show that high innovativeness in the core, due to positive feedbacks among its components connected to each other in a circular structure, enables it to support innovation in peripheral components in a system of different knowledge types.

Economists have used the core-periphery construct to build theoretical models. A model exploring issues of concentration of manufacturing in a 'core' region while the rest of a country acts as the agricultural 'periphery' was developed by Krugman (1991; a version of the model is also discussed in Chapter 5 of Fujita et al. 1999; a coupling of the model with endogenous growth models is attempted by Baldwin and Forslid 1999). My brief discussion here is largely restricted to Krugman's (1991) original coreperiphery model. The model has two regions, each with an increasing-returns

21 Innovation here largely refers to the production of new useful knowledge, the assimilation of which into an economy underlies economic growth. Innovativeness then is the speed with which an actor (or a site) absorbs and produces new ideas or knowledge. 
manufacturing sector and a constant-returns agricultural sector. There are two factors of production: each factor is specific to a sector, i.e. agricultural peasants and manufacturing workers, with the share of workers as $\mu$. Krugman (1991) assumes that preferences of all consumers are the same: determined by a Cobb-Douglas function with $\mu$ as the share of expenditure on manufactures. Also firms in each region are assumed to exhibit profit-maximizing pricing. The peasants are immobile whereas the workers can move between the two regions. The parameters that determine convergence between regions are the share of consumption expenditure received by a sector, elasticity of substitution between the different products of the manufacturing sector, and an index for transportation costs which only apply to the manufacturing sector's output. Krugman (1991) concludes that high transportation costs work against the emergence of a core-periphery structure. A manufacturing core and an agricultural periphery structure emerges if the transportation costs are sufficiently low and if manufacturing gets a slightly higher share initially in one region under the assumption of strong economies of scale. ${ }^{22}$

Now I turn to the internal structure of a core as compared to that of a periphery in a system. In a study on mergers and acquisitions, Green (1987), while adding a fourth category of 'semi-core' to Wallerstein's (1974) core-periphery model, discussed some important structural distinctions between the different categories. According to him, the core possessed the highest density of internal and external connections; the semicore only differed from the core by having a smaller number of internal connections; the semi-periphery had no internal connections but was more strongly connected to the core than to the periphery; finally the periphery had very few connections to other parts of the system. Borgatti and Everett (1999) review three intuitive conceptions of the core-periphery structure in the literature using social network methods: first, all members of a network belong to just one cohesive group but core members may be more 'integrated' into the group than the peripheral members; a second similar view partitions a network into two blocks - a core block and a periphery block; third, the core may be taken as the physical centre of a network if, using an appropriate representation algorithm, it is viewed as a cloud of points in Euclidean space. In Borgatti and Everett's (1999) description of these views, a network can only have one core as shown in Figure 2.2.23

22 The importance of increasing returns in manufacturing is long recognized in economics and was routinely emphasized by classical economists: for example, Adam Smith (1991[1776]) used it as an explanation for the dominant position of England in the eighteenth century.

${ }^{23}$ They discuss multiple cores in a companion article (Everett and Borgatti 1999). 


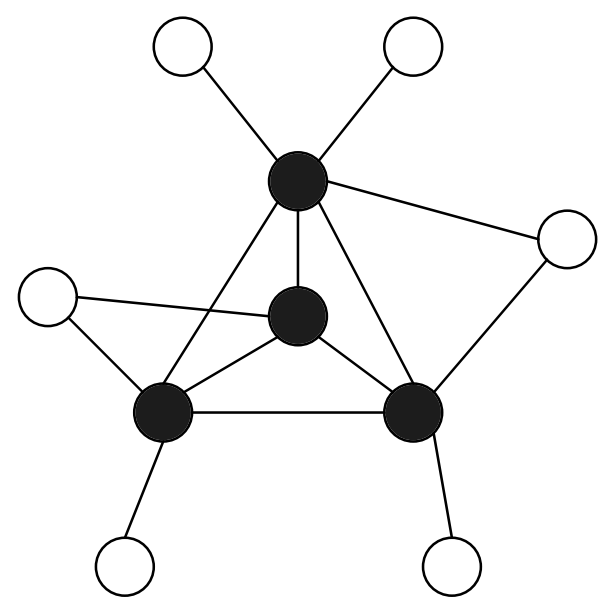

Figure 2.2 Core-periphery structure in a small network (Source: Borgatti and Everett 1999).

Borgatti and Everett (1999) develop a discrete and a continuous model of the core-periphery network structure. In the discrete model, they divide the network (graph) into two distinct classes of nodes: a core whose members are densely connected to each other and a periphery whose nodes are sparsely connected in comparison. A core is basically identified as a cohesive sub-group in a network and the remaining nodes form the periphery. In an idealized version of this structure, members of the core are all connected to each other, peripheral nodes are connected to the core nodes but there are no connections within the periphery. In the continuous model, Borgatti and Everett (1999) assign a 'coreness' attribute to each node in a network. This model avoids the dualistic distinction between a core and periphery, allowing the existence of a semi-periphery and possibly other classes such as semi-cores. Here a comparison to the more common centrality measures in networks reveals that corenodes have high network centrality but the reverse may not be true. According to Borgatti and Everett (1999: 393),

"it is possible to collect a set of the $n$ most central actors in a network, according to some measure of centrality (say, closeness or degree), and yet find that the subgraph induced by the set contains no ties whatsoever - an empty core. This is because each actor may have high centrality by being strongly connected to different cohesive regions of the graph and need not have any ties with each other. ... Our view, then, is that all coreness measures are centrality measures, but the converse is not necessarily true. For example, the betweennessbased measures of centrality (Anthonisse, 1971; Freeman, 1979; Freeman et al., 1991; Friedkin, 1991) will assign high values to actors who are not strongly connected to a core group of people, but who link two otherwise unconnected regions of a network. Coreness measures do not do this."

This difference between coreness and centrality measures becomes clear by comparing two network diagrams, as shown in Figure 2.3. In Figure 2.3a, the set of four filled-nodes in the middle constituting the core possess high degree-centrality values. In Figure 2.3b however, some of the nodes possess high centrality values, 
because they are strongly connected to a small group of nodes in their cohesive subgroup only, but do not form the core of the network as a whole. ${ }^{24}$ Therefore, according to Borgatti and Everett (1999), the main difference between a continuous coreness measure and centrality is that the former provides information about the structure of ties in a network as a whole; whereas the latter only indicates the structure of ties with respect to a specific node, for example, in terms of the graph-theoretic distance of this node to other nodes in a network (Borgatti and Everett 1999).

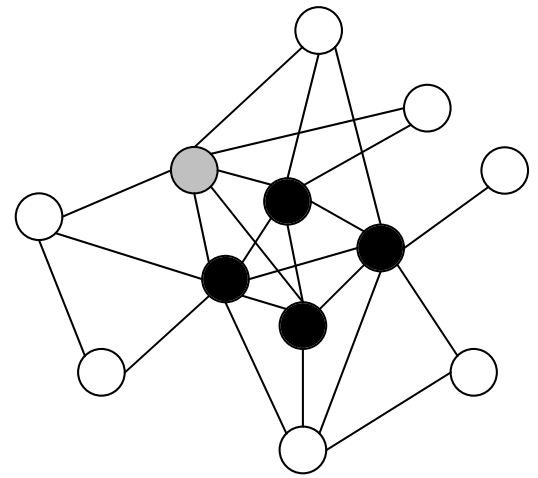

a) Core-periphery structure

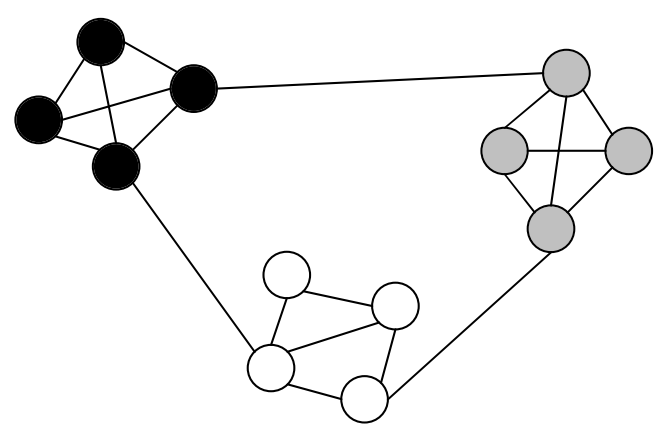

b) Three different cohesive regions

Figure 2.3 Comparison between a core-periphery graph and one with unconnected high-centrality nodes (source: Borgatti 2002).

To summarize the foregoing, according to core-periphery dynamics, a core component may slip into the periphery as a result of its internal conditions and other developments in the periphery. In addition, a core may be distinct from a periphery in a number of dimensions, such as it may be more industrialized or innovative than the periphery. More generally however, a core is a self-supporting structure on which the periphery depends for innovativeness or for growth. In the next section, I discuss how a core becomes self-supporting, in particular, what is it about the internal structure of a core that makes it self-supporting or self-reinforcing? I argue that the circular flow of goods and knowledge between different interdependent actors in an economy possesses qualities, such as positive feedbacks between the actors, that make it selfsupporting.

24 Alternately, if a network can have multiple cores (one for each 'localized region' or clique in the network), then the coreness measure (in combination with a correspondence matrix which identifies a node as being a member of a particular clique or core-periphery complex), tells us whether a node has high betweenness centrality due to its association with members of its own clique or due to its position between different cliques (see Everett and Borgatti 1999). The betweenness centrality measure alone does not allow one to make this important distinction. 


\subsubsection{Circular flow of goods and knowledge: a structural 'tableau' for economic and technological interdependence}

The idea of a circular flow, or a closed-circuit, has been highly influential in economics, in particular to represent the circulation of money in an economy (see for example, Adam Smith's 1991 [1776]: 237 description of money as the "great wheel of circulation"; Marx's 1976 [1867] closed-circuit in the money-commodity-money schemata; the quantity theory equation work by Newcomb 1886; Schumpeter's (1934 [1912]) depiction of self-reproduction of economic activity as circular flow; Foster's 1922 discussion on importance of the circular flow of money for analyses of business cycles; and Knight's 1951 [1933] use of the "wheel of wealth" to illustrate a set of price relations that govern economic systems). The other face of this monetary circular-flow is the circulation of goods and services: discussed by Marx 1976 [1867] as the commodity-money-commodity circuit; and by Newcomb (1886) as the concept of "industrial circulation." The circular-flow concept continues to be important in modern economics and appears in many texts of standard economics (for example, see Samuelson 1970 where the circular flow idea is discussed in many different contexts including price theory; Baumol and Blinder 1985: 114-115 use it for demonstrating the equality between national income and national product; and Mankiw and Taylor 2006 use it to show the flow of goods and services between firms and households in a slightly modified version of Knight's 1951 [1933] circular-flow diagram reproduced here as Figure 2.4).

Below I present a brief review of the theory of the circular flow of goods between horizontally and vertically related actors. I start with an appraisal of two studies whose focus was monetary-circulation and its relationship with the flow of goods. I picked these studies largely because they use illustrative graphical diagrams of the circle to present their arguments. As my main concern here is not the theory of prices or value theory, the discussion is largely restricted to the conceptualisation of the flow of goods in the reviewed studies. Following the discussion on flow of goods, this section ends with an overview of concepts and examples demonstrating the circular flow of knowledge and its central importance in knowledge creation processes where it acts as an innovative core because of the self-reinforcing feedback cycle built into it. ${ }^{25}$

\footnotetext{
25 Unlike circular flow of knowledge (which through its depiction of dynamic feedback loops is an illustration of knowledge exchange necessary for knowledge production or learning by interacting), circular flow of goods basically depicts an equilibrium situation in which production equals consumption under perfect competition. This simple depiction of the economic equilibrium was used by an older generation of economists including Schumpeter (1934), who showed that innovation drove economic life out of self-reproducing circular flow (Harvey and Metcalfe 2005; Nelson 2008). But it has lost currency in recent neoclassical economics, where the idea of economic equilibrium remains popular, with its advanced mathematical models. Thus, most studies on the circulation of goods reviewed in the following are relatively old. The outdatedness of these studies is however not a problem for my present purpose of getting to an exploration of circulation of knowledge by way of circular flow of goods.
} 


\section{Circular flow of goods}

An important graphical representation of the circular flow concept was developed by Knight (1933 [1951]) who used it to illustrate some facets of price theory (also see Patinkin 1973 on the possible origins of Knight's circular-flow diagram or the "wheel of wealth"). Knight (1933 [1951: 59-62]) used the circular flow diagram to illustrate the organization of production and distribution in a 'free-enterprise' economy. His diagram is reproduced below as Figure 2.4. The diagram shows "the exchange of productive power for consumption goods between individuals and business units, mediated by the circulation money": individuals and families on one side of the circle sell their productive services to business enterprises located on the opposite side, and use the "money income thus obtained" to buy consumption goods and services from the enterprises (Knight 1933 [1951: 60]). For the present purposes however, only a part of Knight's use of the circular-flow concept pertains to the flow of goods, shown as the dotted inner circle in Figure 2.4.

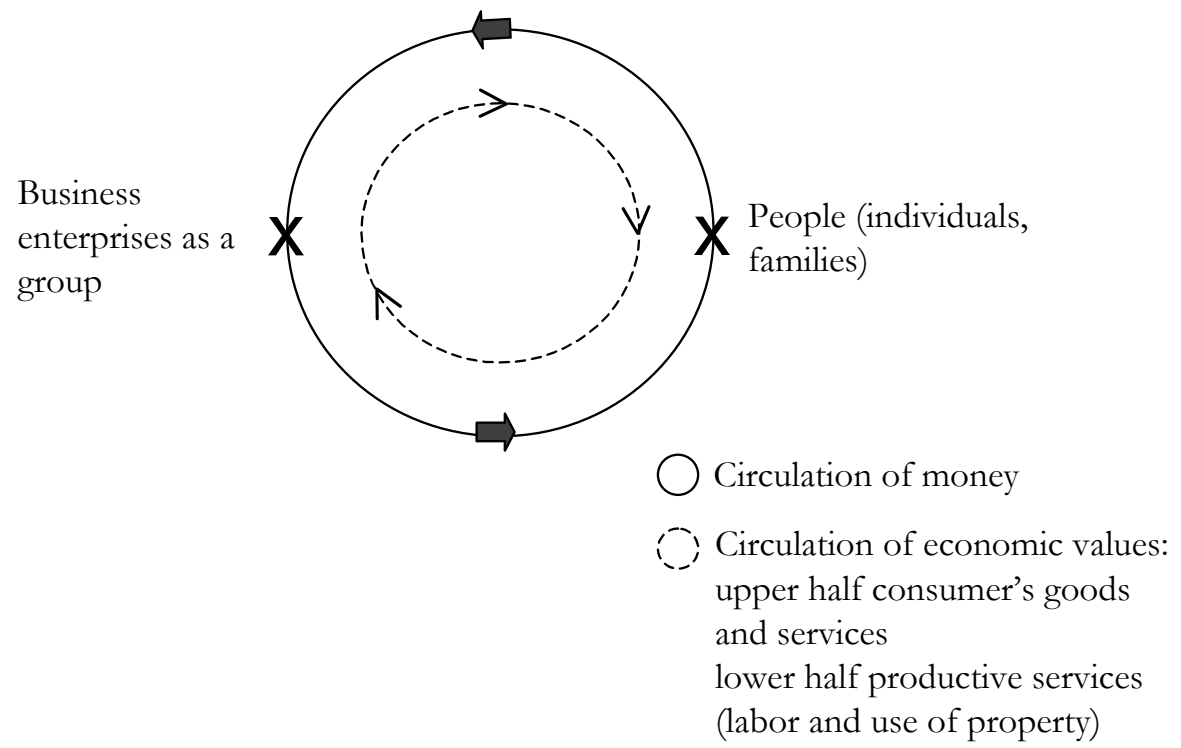

Figure 2.4 Circular-flow diagram depicting the flow of money and values in an economy; reproduced from Knight (1933 [1951: 61]).

Another interesting graphical representation of the circular flow concept was developed by Newcomb (1886) in his work on the quantity theory equation. Newcomb (1886: 318) used network diagrams (digraphs) to show the flow of money between individual members of a "social organism." I quote his description of the graphical representation,

"The conception of the flow of the currency is represented graphically in the following way: We draw a little circle for each person legally capable of being an owner of wealth. Whenever a payment of money is made we suppose it to pass from the circle representing the payer to that representing the payee through a little vein. This vein we represent by a 
line from one circle to the other, with an arrow-head showing the direction of the payment. These veins form a network through which we suppose the money to be flowing from person to person. This continual flow of money from owner to owner is called the monetary circulation.” Newcomb (1886: 318-319, emphasis in the original).

Each of the veins representing the flow of money involves a flow in the reverse direction of "some object of desire, the product of human labor." (Newcomb 1886: 326). The flow of these products is referred to as "industrial circulation" and the combination of the two opposite industrial and monetary circulations is "societal circulation." (Newcomb 1886).

Newcomb (1886) used a set of simple network diagrams to represent monetary flows between different classes in a society; I reproduce some of these digraphs in Figure 2.5a and b below (see the brief descriptions next to the Figure labels). In both diagrams, the node called society "represents the totality of taxpayers, bond-holders excepted;" in 2.5a, tax payments are reflected by directed edge from the society node to the public treasury node. Note that in these diagrams, there are many circular structures or cycles in graph theoretic terms, and some smaller cycles exist within the larger ones. More importantly, all cycles terminate in the 'society' node. The society node depicted in these diagrams is a 'black box' containing many networks of the type shown here. If the 'black box' of society is un-packed, potentially a much richer set of cycles will emerge. In an empirical chapter of this thesis, I present three different networks (social, business and knowledge) existing in a 'society', a village in Andhra Pradesh, India, where a rich set of cycles is observed in all of the networks.

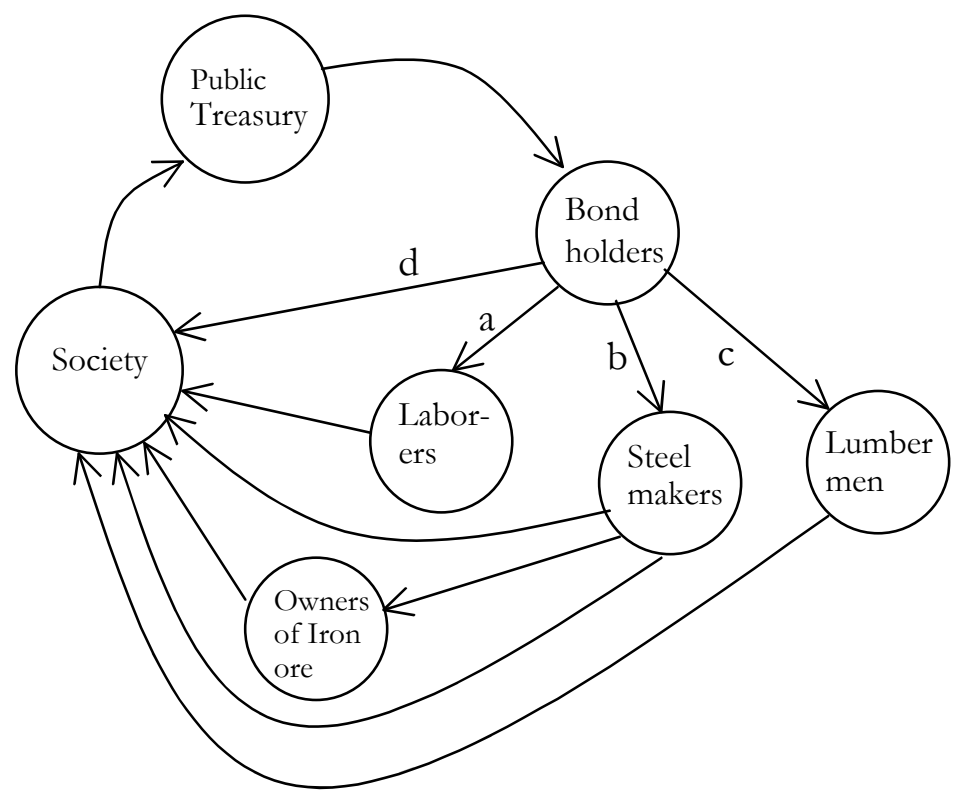

Figure 2.5a Diagrammatic representation of flow of money from the public treasury (equal to the sum of the four flows a, b, c, d going out from the bond holders). Source: Newcomb (1886: 335). 


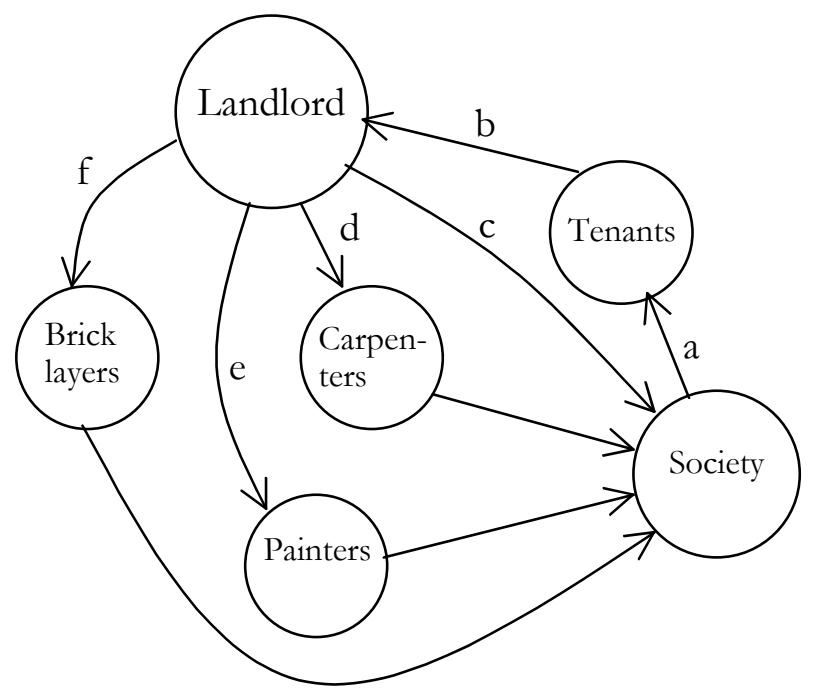

Figure 2.5b Diagrammatic representation of flow of rents to a landlord from his tenants, b, which the former spends for his own support, c, and building more houses, d, e, f. The tenants' income, a, comes from the "society at large." Newcomb (1886: 336).

Origins of Newcomb's (1886) and most other economists' conceptualisation of circular flow of economic life can be traced back to the French physiocrats. In fact, it is widely believed that the first systematic discussion of circular flow of goods in an economy is due to the French physician Francois Quesnay and his fellow physiocrats from the 1750s and 1760s (Schumpeter 1912 [1934]; Spengler 1945; Nordhaus 1992). According to Spengler (1945: 196), writers before the physiocrats did not posses a "sufficiently clear conception of essentially circular and continuous character of the economic process;" and were unable to resolve "economic life into its two component streams - that of goods and that of money, each moving in an opposite direction." 26 Quesnay in his famous Tableau économique from 1758 described the circulation of goods and income between different classes in an economy. His tableau showed flows between a productive class of farmers, miners and fishermen; "sterile" manufacturers, artisans, distributors of commodities; and landlords and others paid out of proprietary revenue (Spengler 1945; Schumpeter 1912 [1934]; Nordhaus 1992). The cultivators, i.e. the productive class, are the only group which produces a surplus in this scheme: they

26 Spengler (1945: 196) adds that the physiocrats attempted to study the "determinants of the behavior of these two streams ... only to fail in part - largely because of their angleworm theory of production and their disregard of the entrepreneurial function. Even so their analysis revealed the circularity of the economic process and posed problems which led to the formulation of Say's law of markets and prepared the way for both Marx's scheme of flow and that now associated with the name of Lord Keynes." 
pass their 'net product' to the landlords who are not considered 'productive' by the physiocrats. The sterile class satisfies its own needs for food etc. by producing goods and services of sufficient monetary value (Spengler 1945). According to the physiocrats, this circulation of money, goods and services between the classes was a never-ending process even though their calculations in the tableau were in annual aggregates. This sense of continuity of flows without any changes in production methods and consumption habits reduced the physiocrats' analysis to an essentially static process (Spengler 1945; Marx 1976 [1867]). Further, the physiocrats never presented their circular flow theory for exchange among individuals: restricting it to the flow between different classes alone (Spengler 1945).

According to Nordhaus (1992: 844), Quesnay's main contribution to economics was an exposition of the 'interdependence of economic life'. Schumpeter, on the other hand, attaches greater importance to the work of Quesnay and other physiocrats, rating it as the foundation on which future economic analyses were built,

“... the Physiocrats or 'Economists' who made the great breach, through which lay all further progress in the field of analysis, by the discovery and intellectual formulation of the circular flow of economic life." (Schumpeter 1912 [1934: 43]).

Schumpeter (1912 [1934: 44]) considered the Physiocrats the "first genuine economic 'school', since only a total conception could form the basis for one." 27 The depth of Schumpeter's regard for the Physiocrats can be gauged by the fact that he starts his classic work, The Theory of Economic Development, with a chapter on the circular flow of economic life. More recently, some authors have attempted to interpret Quesnay's tableau économique as a Leontief system (see for example Maital's 1972 and Samuelson's 1982 open-system linear formulations; and closed-system articulations which take the physiocrats' circular flow concept into account by Phillips 1955 and Bilginsoy 1994).

The classic works of Adam Smith, David Ricardo, and Karl Marx were influenced by the physiocrats (Schumpeter 1934 [1912]). Smith (1991 [1776]) developed a coherent synthesis of the various strands of economic thought of his time, by adding the concepts developed by the physiocrats to the ideas he inherited from his Scottish peers. Ricardo, in a similar way to the physiocrats' emphasis on the net product of cultivators or the productive class, ascribed a dominant role to the profit of the farmers on which the profit of all other sectors of an economy depended. Marx (1976 [1867]) borrowed many concepts from the physiocrats to develop his idea of 'simple circulation' as in the commodity-money-commodity (C-M-C) closed circuit scheme. As an illustration, Marx offered the example of the weaver who sells his linen for two pounds and then buys a bible for exactly the same amount of money. Here the first commodity is sold for money that is used for procuring another commodity, thus completing the $\mathrm{C}-\mathrm{M}-\mathrm{C}$ circuit.

${ }^{27}$ Further, in his History of Economic Analysis, Schumpeter (1954) considered the use of circular flow idea central to the development of the science of economics. 
Schumpeter viewed the circular process as a central phenomenon in economic exchange. His conceptualisation is illustrated in the following,

"How much meat the butcher disposes of depends upon how much his customer the tailor will buy and at what price. That depends, however, upon the proceeds from the latter's business, these proceeds again upon the needs and purchasing power of his customer the shoemaker, whose purchasing power again depends upon the needs and purchasing power of the people for whom he produces; and so forth, until we finally strike someone whose income derives from the sale of his goods to the butcher." (Schumpeter 1934 [1912]: 7).

This return to the butcher in the above chain completes the circular flow of goods. According to Schumpeter, every individual contributes to the social process of production in order to get their share of the total output: here each share received from the total output involves a corresponding contribution as productive labour, closing the circular flow in this system. Note that Schumpeter's and Marx's concept of circular flow of commodities is abstracted away from specific goods. Below, I discuss the possibility of circular flow of goods in a more specific setting in terms of the use of goods to produce goods i.e., between the different stages of a production chain of a particular good.

In earlier sections of this chapter, in particular $\$ 2.1 .3$ and $\$ 2.1 .4$, I discussed how knowledge interdependence arises due to vertical relationships between different actors in a production network or chain. Here I concentrate on the flow of goods between these vertically related actors. More precisely, my aim is to demonstrate that, in addition to the expected linear flow, circular flow of goods may take place between some vertically related actors. Such a circular flow of goods arises when goods, as machines, are used to make other goods. And the latter goods then act as an input to the production process of the first. This nature of circular flow between vertically related actors has been considered central to the development of industrial production by various authors (see for example, Marx 1976 [1867]; Sraffa 1960; Pasinetti 1980). According to Marx's (1976 [1867]: 506]),

"Large-scale industry therefore had to take over the machine itself, its own characteristic instrument of production, and to produce machines by means of machines. It was not till it did this that it could create for itself an adequate technical foundation, and stand on its own feet." (emphasis added).

For the present purposes, the "adequate technical foundation" was made possible by the circular process of machine-production by machines, which allowed large-scale industry to "stand on its own feet." Note the elements of self-support and cumulativeness implicit in the quoted words. Later in this section, I show that the same two elements are crucial in circular flows of knowledge between economic actors, and the model presented in the next chapter formalizes these two concepts for a system of different knowledge types. If the development of a technological system is represented as an evolving network of technologies or knowledge types, then in a core-periphery scheme this circular process may act as the core that provides the foundation on which the network is built and allows it to be sustainable. 
Consider a scheme with three actors, a good $\mathrm{x}$ (a component in an assembled product) produced by an actor $\mathrm{X}$ is passed to another actor $\mathrm{Y}$ who transforms it into another good $y$ and passes it to an actor $Z$ who modifies it further by adding more components or performing more processes on it. Finally $\mathrm{Z}$ produces the good $\mathrm{z}$ and passes it to $\mathrm{X}$ because $\mathrm{X}$ uses the good $\mathrm{z}$ in the production process of its own good, $\mathrm{x}$. Here each node in this $\mathrm{X}-\mathrm{Y}-\mathrm{Z}-\mathrm{X}$ circle is performing a production process of some sort which modifies the initial component produced by $\mathrm{X}$ in various stages to produce an input for the production process employed by X. Capital goods, and other generic technologies, are probably the easiest and best examples of this production of goods by means of other goods. Rosenberg (1976) uses the history of the machine tool industry in the United States to illustrate some important aspects of this phenomenon. In the $\mathrm{X}-\mathrm{Y}-\mathrm{Z}$ example above, assuming that $\mathrm{X}$ produces a component of a machine tool which goes through multiple stages to become a machine tool i.e., the good produced by $\mathrm{Z}$, which is once again used by $\mathrm{X}$ to produce its product. For example, lets say that alloy-steel gears used in the transmission of drive and feed mechanisms of a machine tool are manufactured by $\mathrm{X}$, which are then used by $\mathrm{Y}$ to make the drive and feed mechanism assembly for supplying to $\mathrm{Z}$. The latter is a machine tool manufacturer who supplies complete machine tools to $\mathrm{X}$ for use in the production process of the alloy-steel gears, thus completing the circular flow of goods (see Rosenberg 1976: 28 for the origins of this example where he uses the same component-assembly scheme to make a different point about the interdependence between machine tool and automobile technologies). The above example remains valid even if $\mathrm{Z}$ produced a component of a machine tool, instead of the finished assembly, as long as $\mathrm{X}$ uses $\mathrm{z}$ to manufacture its product. Similar examples of the circular flow can be easily found for other general-purpose technologies such as energy systems, automobile, and the various communication technologies. For goods with fewer backward and forward linkages, a circular structure in the flow of goods within their production chains may be more difficult to locate. ${ }^{28}$

\section{Circular flow of knowledge}

Discussions of possible circular structures in knowledge flows are not as common in economics as those of the circular process in the flow of goods. Neither does the idea of the circular process in knowledge flows have as impressive an history as the concept of the circular flow of goods or money. However, as a real-world phenomenon, circular flow of knowledge is probably more prevalent in an industrialised economy than its counterpart in goods. Moreover, the two phenomena are related to each other because a large portion of knowledge flows through the same

\footnotetext{
${ }^{28}$ In other words, circular flow may be a more common phenomenon for generic technologies which are considered crucial for economic growth as highlighted by many authors, see for example, Grossman and Helpman (1991); and Bresnahan and Tratjenberg (1995). In a technological system consisting of different goods and the set of actors manufacturing the goods, a circular structure in the flow of goods is likely to be associated with the more-important generic technologies. Using the terminology discussed in section 3.1, the circular process may be treated as a core of the system.
} 
channels as goods of the same technology. Although the precise relationship between the structures of flow of goods and those of knowledge flow is far from obvious. In a later chapter of this thesis, I present a comparison between the knowledge-network of an Indian village to its business network. In the present discussion however, I briefly discuss the current dominant model of the innovation process that necessitates a rich collection of circular flows of knowledge in an economic or technological system. Then I discuss aspects of feedback systems among interconnected actors, which bring about a circular flow of knowledge. ${ }^{29}$ Subsequently, I look at flows of knowledge and ideas in an economy due to technological interdependence, as discussed in the examples presented in $\$ 2.1$ of this chapter. However here I present a more dynamic picture of technological change and the innovation process by using elements from patterns of stability and discontinuity in technological evolution discussed in $\$ 2.2$.

The social-scientific view of the innovation process has undergone significant changes over the last few decades, entailing an equally significant change in the way knowledge flows are envisioned. Until the 1960's, the dominant model was a linear one which started with institutions of basic science, feeding into applied research and development, and ending in production or diffusion i.e. commercialisation of technology (Godin 2005). ${ }^{30}$ This linear model was challenged in the early 1970 s by economists who proposed the so-called need-pull model: this model started with market as the source of ideas to which R\&D reacted (Rothwell, 1992). According to Rothwell (1992: 236), these two generations of models were succeeded by three others and we are currently in the fifth generation i.e., the "systems integration and networking model (SIN)." The SIN model, like the systems of innovation framework briefly reviewed in \$2.1.4, underlines "strong linkages" with users and "strategic integration between collaborating companies," which point to a vast network of knowledge flows between heterogeneous actors. If one could get a bird's eye view on this network, a rich web of circular processes of knowledge flow would be revealed.

The circular flow of knowledge (or information) begins through feedback loops in systems: feedback among actors creating a circular process is considered an essential feature of knowledge flows in an innovation system (see Mackinnon et al. 2004 for an empirical illustration using information from the Aberdeen oil complex; for a theoretical exposition of multiple circular feedback loops in a systems simulation model, see Galanakis et al. 2000). Studies on cybernetic systems and communication theory in the 1940's were probably the first to discuss feedback, or circular loop in the context of information flows, among system-components (see for example, Wiener

\footnotetext{
${ }^{29}$ Here it may be illustrative to distinguish between knowledge and information for discussing flows and feedback effectively. More precisely, I must build on the brief discussion from the previous chapter on the distinction between codified and tacit components of knowledge. For the present discussion however I refer the reader to the following important references: Nelson and Winter (1982, ch. 4); Cowan et al. (2000); and Nightingale (2003).

${ }^{30}$ Godin (2005) demonstrates that the linear model of innovation remained in currency much longer than generally assumed by many prominent students of technological change.
} 
1948; Shannon and Weaver 1949). However, the basic concept of feedback has its roots in the circular flow arguments of the physiocrats discussed earlier in this section. According to Patten and Odum (1981: 886), cybernetic systems are

"a special class of cause-and-effect (input-output) systems in which input is determined, at least in part, by output. The portion of output that is returned to input is the feedback, and this may become the basis for feedback control."

Further, Patten and Odum (1981) distinguish between two types of simple systems with feedback. The first type is a determinate system whose "behavior is governed only by past causes" and the second type is a control system that is "teleological because its behavior is guided by future or desired goals." (Patten and Odum 1981: 886). The two types of systems are shown as Figure 2.6 (a) and (b) respectively. The crucial point for the present purpose is that cybernetic systems possess a closed information network, i.e. all system components are interdependent and there are many circular feedback loops for information flow in the system.

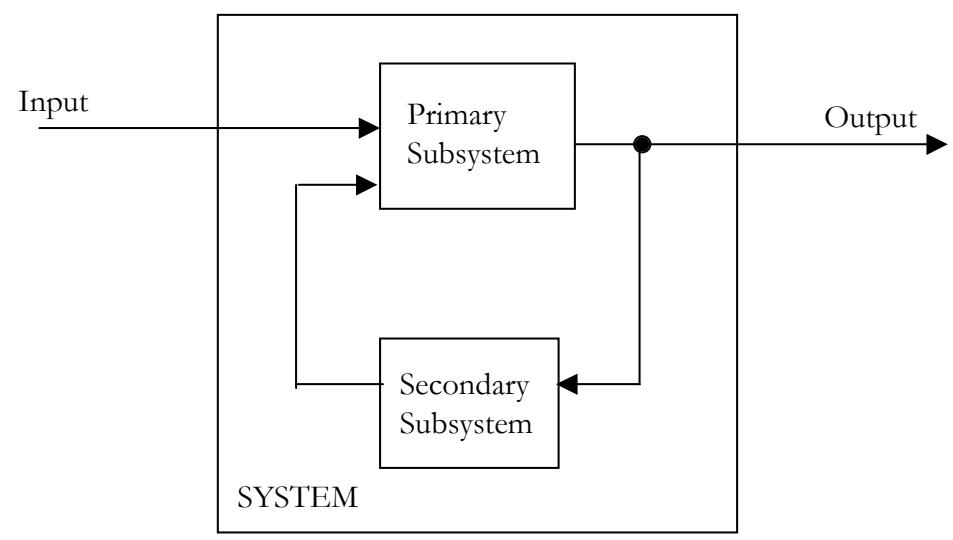

2.6 a)

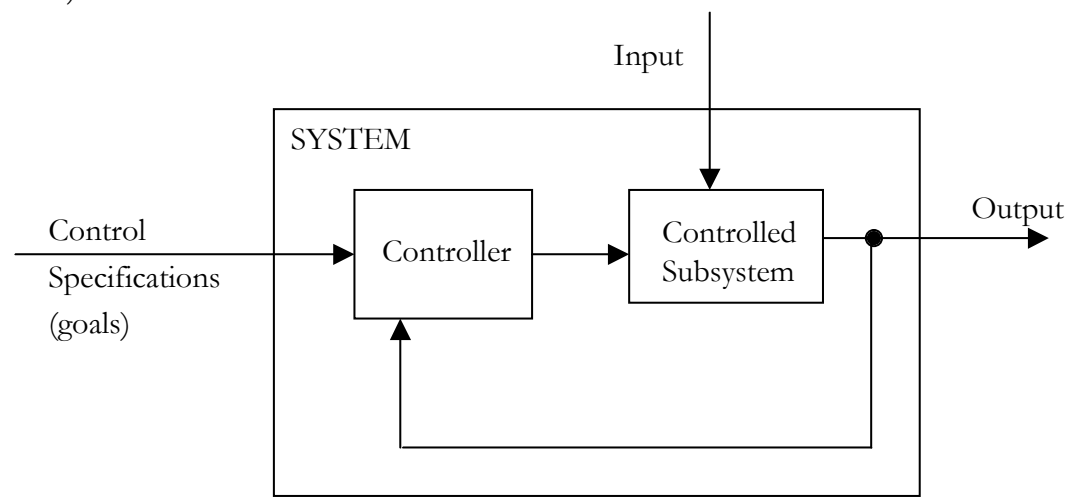

b)

Figure 2.6 Two types of cybernetic systems; reproduced from Patten and Odum (1981). 
A second stream of studies focussing on feedback systems came out of engineering, in particular electrical engineering. These studies were adapted to social sciences by the "industrial dynamics" group working at MIT's Sloan school of management in the 1950s and 60s. According to an important proponent of industrial dynamics, Forrester (1968a: 402),

"The basic structure of a feedback system is a loop within which the system condition provides the input to a decision process that generates action which modifies the system condition. It is a continuously circulating process."

Note the notion of continuity here which is the same as that emphasized by the physiocrats. Further, Forrester (1968a; 1968b) points out that system behaviour depends upon the structure of a system, which in turn begins with the structure of the loops. In other words, two elements are important for system behaviour: the internal characteristics of a feedback loop and the interaction between the different loops if more than one loop exists in a system. ${ }^{31}$

For the present purposes, the most interesting aspects of feedback systems are those of feedback polarity in terms of positive and negative, and the continuously circulating nature of the feedback process. Negative feedback is a commonly studied phenomenon in conventional economics where deviations from a stable equilibrium position are a major concern: negative feedback brings the system back into equilibrium each time it's nudged out of it. Positive feedback, with its tendency to keep a system perpetually out of equilibrium, has been largely neglected in economics before the 'advent' of the new growth theory and the new trade theory in the 1990's barring a few exceptions in the cumulative causation and the technology competition literatures (see Myrdal 1957 and Kaldor 1972 for cumulative causation in economic growth and development; and David (1985) and Arthur (1988) for technology competition in an increasing returns setting). The focus in this thesis is largely on positive feedbacks, particularly in the context of accumulation of technological knowledge. In a model presented in the next chapter, positive feedbacks arising out of auto-catalysis in a circular loop drive system dynamics: the circular loop acts as a core of a system of different knowledge types around which further construction of the system takes place. This definition of core as a set of nodes in a circular loop is similar to Forrester's (1968: 408) who views the "feedback loop as the basic structural element of systems."

31 In an article on information flows within an organization, Goldman (1959) structures the organization as a hierarchical pyramid, starting with the general manager at the highest level and the operators at the lowest. Superimposing a communications model on this structure, Goldman (1959: 272) points to a circular flow of information as: "Data are generated by operators and transferred (or transmitted) as one figure for the group by a monitoring (or filtering) device to the first level of supervision; the group figures for the first level are combined again etc. for the use of a higher management level and then fed back to down through the pyramid - in a "circular" fashion." Therefore, circular flow between subsystems may form an integral part of knowledge flows occurring in a hierarchical system, such as the one shown in Figure 2.1. 
Below I discuss knowledge flows between different actors involved in the development of new technologies, akin to the discussion in $\$ 2.1$ of this chapter. Further, building on the temporal notions developed in $\$ 2.2$, here I provide historical examples in a more dynamic co-evolutionary setting to demonstrate the importance of the circular flow structure in knowledge creation processes. As a result, rather than talking about interactions between technologies, I discuss knowledge flows between different innovations (or inventions) that feed into each other: ${ }^{32} \mathrm{I}$ demonstrate that the process of knowledge creation occurs in an auto-catalytic fashion where one novel idea or invention generally leads to another (Metcalfe 2000).

\section{Circular knowledge flows in the innovation process}

The description of the examples below is based on the assumption that technological invention is a gradual evolutionary process involving many small incremental steps (see for example, Usher 1954; Simon 1981; Mokyr 2002).

"In the concept of process, the emergence of novelty is presented as an accumulation of many individual items over a relatively long period of time. The magnitude of the individual item is small, but through cumulative synthesis the product becomes important." (Usher 1954: 61).

Silverberg and Verspagen (2004) have termed these incremental steps in the innovation process as 'sub-innovations' and provide the example of a bicycle which was developed through numerous sub-innovations over the course of several decades. Further, the decentralised processes of gradual knowledge creation and accumulation, that lie at the root of technological innovation, generally involve social interaction among a large group of heterogeneous actors working on a set of interdependent technologies: according to Rosenberg (1982), individual innovations are almost always embedded in a network of interrelated innovations. Below I provide some examples to demonstrate this continuity, and interdependence, in knowledge creation over time starting with an illustrative example of linear flow from invention to invention. Following this I discuss some historical examples with the aim of identifying a circular structure of knowledge flows between more than two inventions (or more precisely between actors who developed these inventions). Finally, I attempt to demonstrate the central importance of the circular flow of knowledge in the innovation process: I outline elements of a hierarchy of combination structures of incremental innovations according to which the circular process may act as the core around which a network of interrelated peripheral inventions is built. This phenomenon is illustrated in the brief example about the telephone below.

The inventor par excellence, Thomas Edison, once acknowledged that his work on the telephone was built upon the idea of the Reis musical telephone which Philipp Reis, a German schoolteacher had demonstrated to the public in 1861 (Carlson 2000). Working from 1875 to 1877 , Edison transformed the Reis telephone by using ideas

32 They may or may not succeed each other in time as the first invention can always be improved upon due to a knowledge flow from the third. 
from the electromechanical apparatus developed by Helmholtz to produce vowel sounds and his own patent on the liquid rheostat from 1873 (Carlson 2000). Edison eventually secured ten patents for his work on the telephone. There are three important things to note in the above example: first, the various incremental innovations were built upon knowledge from diverse fields. Second, a synthesis of various steps is generally recognised as a unitary radical innovation ex-post; radical innovations are "made possible by numerous minor innovations." (Sahal 1981: 20). Third, the gradual emergence of a radical innovation, as a combination of minor innovations, initiates a new era of systemic technological development, as discussed in \$2.2.2. In a systems context, this combination becomes the core of a network of interrelated inventions, forming "the basis around which a larger number of further cumulative improvements and complementary inventions" are built (Rosenberg 1982: 59). These latter complementary inventions then act as the periphery in this network of inventions. However, this systemic accumulation of knowledge may later be disrupted by a new radical combination that initiates technological development along a new path.

Now I turn to some examples of circular flow of technological knowledge between a small number of heterogeneous actors and their different knowledge types or technologies. In general, the circular process of knowledge flow between distinct inventions is a natural outcome of the incremental step-by-step pattern of the distributed innovation process. The reciprocal interactions between technologies discussed in \$2.1, for example between the steam engine and the machine tools, refer to the simplest, and the smallest two-node, circular flow of knowledge. ${ }^{33}$ In the examples presented below, I focus on identifying circular flow of knowledge between three or more actors. The examples highlight two types of circular flows between innovations. The first arises out of the catalytic process of inventions leading to more inventions and the circular combination of inventions acting as a basis on which

33 This phenomenon of dyadic interactions may be extended to a set of three or more technologies. Consider the following example with knowledge flows between three technologies: the dyadic interactions between the singer sewing machine company, the Babcock and Wilcox company and the machine tool manufacturers, discussed in $\$ 2.1 .3$. This three-node bidirectional circular flow of knowledge is depicted in the Figure below. A similar three-way relationship existed between machine tools, the bicycle and the automobile at various stages of their development, in particular at the beginning of the $20^{\text {thm }}$ century, as noted by Hounshell (1984) and Rosenberg (1982) (see $\$ 2.1 .3$ above for a detailed discussion).

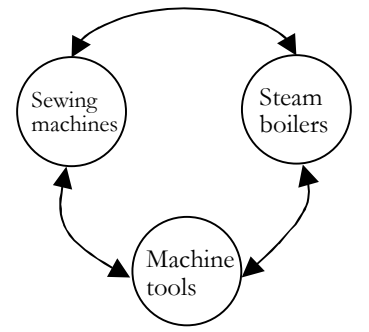


further complementary inventions are built. The second type of the circular process emerges out of the flow of knowledge between vertically related actors who collectively develop a new technology or product.

In the first place, circular flow between three different actors accredited with three different inventions (or groups of inventions/patents on exactly the same process) arises when one invention leads to a second, the second leads to a third and finally the third leads to a direct improvement in the first. Consider an important invention in chemistry, electrolysis with the Hall-Héroult process, which made the separation of aluminium from its ore (aluminium oxide or alumina) commercially viable after Charles Hall's first patent in 1886. However it was soon realised that alumina that is electrolysed to produce aluminium must be free from impurities. Hall then developed a purification process for alumina and filed at least four patents on the purification of alumina during 1900-01 (Meiers 2006). Following this in 1902 he submitted patents on the manufacture of carbon electrodes and a stopper for electrolytic pots. These last set of patents directly improved upon the electrolytic process he first patented in 1886 and completed the circle of knowledge flow between the inventions mentioned here. This circular process is graphically shown in Figure 2.7 below.

A similar process of circular flow is observed if one traces Hall's patents on the manufacture of alumina from the unrefined ore of bauxite: The first of these patents was filed in 1900 and the last one in 1909 which was an improved process for manufacturing alumina. In between these two patents that complete a circular process, Hall developed a process to make paint in 1902, the carbon electrodes and stopper for electrolytic pots mentioned already, and some other processes of purifying alumina. On the surface, the flow of knowledge between the above inventions is taking place in the head of one inventor. But an investigation of the changes in the locus of these inventions, over the course of two decades discussed above, reveals important organizational developments. Before 1886, Hall was experimenting in a rudimentary laboratory with his sister in Oberlin, Ohio. In 1909, on the other hand, he was working at one of the most successful aluminium production units in the world, which was later to become the largest aluminium manufacturer in the United States, ALCOA.

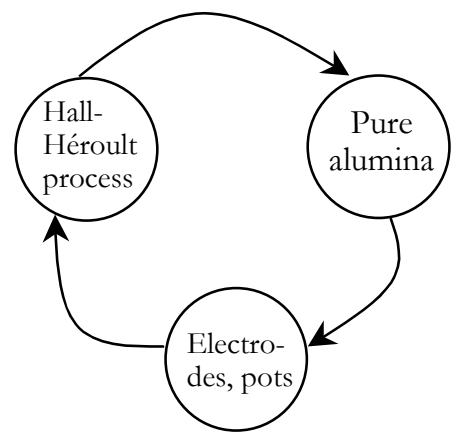

Figure 2.7 Circular process of three inventions in aluminium production. 
Other examples of circular processes between inventions, according to which a later invention several incremental steps after the first invention carries in it important knowledge about the improvement of the first innovation, can be found in the history of technology (the works of Usher 1954; Hounshell 1984; Hounshell and Smith 1988a; Bijker 1995; and Mokyr 2002 may provide useful starting points for these investigations). Moreover, as pointed out by Rosenberg (1996), a later invention feeding into the first in a circular process may come from a completely unrelated field of knowledge or industry. For example, explanations for and many useful improvements in the process of age-hardening that led to the discovery of the duralumin alloy by Alfred Wilm in 1903 remained obscure until the development of the seemingly unrelated field of electron microscopy. The details of the precipitation processes observed through the electron microscope, in the case of duralumin, have been used to strengthen many alloys (Mokyr 2002). A final important point: in addition to the circular process in knowledge flow among inventions, another essential point is witnessed in the above examples relating to the gradual assembly of a radical innovation. In the aluminium example, the radical innovation of process of separation of aluminium from its unrefined ore was actually a collection of 'minor' inventions by Hall and other inventors. This collection, in the form of a circular process as discussed above, formed the core of the network of interrelated inventions that led to the effective development of the aluminium manufacturing process.

Turning to knowledge flows among vertically related actors, or knowledgeinteractions in user-producer relationships: a circular structure between three such actors $\mathrm{A}, \mathrm{B}$ and $\mathrm{C}$, comes to the fore when $\mathrm{C}$ who is only indirectly related to $\mathrm{A}$ gives feedback to A about a product they are collectively developing. For example, consider the development of a plastic heat exchanger where firm $\mathrm{A}$ is involved in the development of a suitable plastic material, a firm B is developing the process of extruding the plastic into thin tubing required for the heat exchanger, and a firm $\mathrm{C}$ develops the heat exchanger assembly including the successful attachment of the header components to the new types of tubing received from $\mathrm{B}$ and testing the heat exchanger for efficiency requirements. Here for effective development of the innovation i.e., the heat exchanger, firm $\mathrm{C}$ must give feedback to firm $\mathrm{A}$ about the performance of the assembled heat exchanger, particularly in terms of suggestions about the manufacturability and high-temperature resistance of the plastic (see von Hippel 1988 for different case studies where 'users' either acted as innovators or provided critical inputs to the innovation process). This feedback from the user-firm $\mathrm{C}$ provides A with important knowledge about further development of the plastic material and completes the circle of knowledge flows between firms A, B and C. Similar structures can easily be identified between actors defined at different levels such as industries, sections of firms, or individuals. As an example at the industry level, once again consider the Hall-Héroult electrolytic process or, in general, electrolysis: an invention coming out of the chemical industry (actor A), which was crucial in the metallurgy industry (B) for the reduction of many metal ores. The wide use of 
aluminium, for example, in different industrial sectors to a large extent had to wait the development of hardened aluminium alloys. The first important aluminium alloy was duralumin developed through a process called age hardening by the German metallurgist Alfred Wilm in 1903. Duralumin in turn played an important role in the development of aircrafts because of its properties of high strength and good machinability. In particular, it was used by Count von Zeppelin and Professor Junkers to construct airships in the fledgling German aircraft industry (C) (Polmear 2004). ${ }^{34}$ Many further improvements in aircrafts, particularly in the engine, turbine and wingdesign arenas, have been crucial in the development of the chemical industry i.e. the actor $\mathrm{A}$ in this example, thus completing the circle of knowledge flows between the three industries discussed. ${ }^{35}$

Another related issue in the circular process analogy is the flow of innovation benefits between actors. Once again exploring the example of aluminium production using the Hall-Héroult process: separation of aluminium using direct reduction with carbon was difficult because of the high melting point of aluminium oxide. Normal electrolysis using sodium or potassium salts for preparing the electrolytic bath did not work because aluminium oxide did not dissolve in the salt-solution. The breakthrough of Charles Hall and Paul Héroult with the Hall-Héroult process was to prepare the bath using cryolite: in Hall's original patent filed in 1886 the bath solution is described as a fusion of fluoride salts of aluminium and Sodium or Potassium (Meiers 2006). The availability of low-cost and high purity aluminium as a result of the Hall-Héroult electrolytic process gradually brought about a revolution in the metallurgy industry. Around the same time, in a similar fashion to aluminium availability of other metals such as manganese, chromium and tungsten was also made commercially viable as a result of electrolytic processes (Rosenberg 1982). Therefore, metallurgy as a field benefited tremendously from the invention of electrolysis in chemistry. The refined metals such as aluminium became easily available to the electrical industry for use in transmission cables as a replacement for the expensive copper: aluminium gradually became the preferred metal for high-voltage overhead transmission and is also used in underground transmission lines. The use of low-cost aluminium instead of copper reduced the costs of electric transmission, which in turn benefited and improved the cost-efficiency of the Hall-Héroult electrolytic process in which the cost of electricity forms a large percentage of the total cost: $25 \%$ in the modern Hall-Héroult process (Welch 1999). The example here forms a circular process of innovation benefits across actors as shown in Figure 2.8.

\footnotetext{
34 The first passenger aircraft came out of Junkers factory in 1919, laying the foundation for both the airline industry and a civilian aircraft industry.

${ }^{35}$ Here it is obvious that identifying the circular process of knowledge flows between industries is easier than that between a set of lower level actors such as individuals. This is because at higher levels of aggregation, such as industry, one can easily abstract away from very specific types of knowledge because industries generally possess a broader knowledge base than individuals.
} 


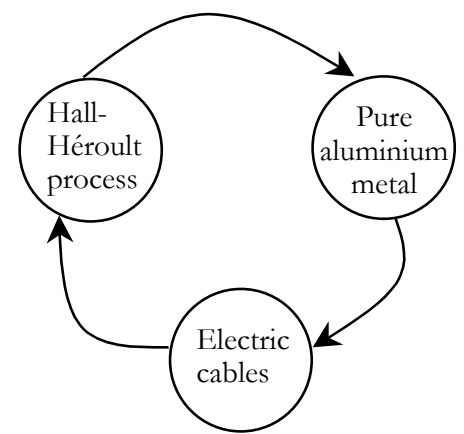

Figure 2.8 Circular flow of benefits between three different innovations.

Finally at a more general level, the simplest form of circular flow of knowledge in the form of bidirectional user-producer interaction among actors in a production chain - is the basic idea underlying the concept of innovation systems (Lundvall 1985; 1988; Freeman 1995; see \$2.1.4). The user-producer interactions are generally embedded in an innovation system where firms have a network of relationships with non-firm actors such as those from academia and government. In this innovation architecture, the institutional spheres of industry, government and academia overlap with each other, and interactions between actors from the three spheres generate a triple helix structure composed of numerous circular flows of knowledge and expectations (Etzkowitz and Leydesdorff 2000). On the whole, in the SI literature, circular flow of knowledge is believed to be an important widespread phenomenon (see for example, Lundvall 1985; 1988; 1992; Freeman 1995; Etzkowitz and Leydesdorff 2000; Lundvall et al. 2002; Asheim and Isaksen 2002).

Thus a circular process of knowledge-flows may be considered to be a basic factor in defining an innovation system or, in purely technical terms, a system of technologies and knowledge-types. Then to have a coherent dynamic system (or subsystem), one must be able to identify at least one circular process in its structure. In a world of multiple overlapping systems, the existence of a circular process in a web of interconnections may be instrumental in reducing the ambiguity involved in defining the boundary of a system (or subsystem), by acting as a necessary condition for the existence of a system.

Further, the feedback element existing in the circular flow of knowledge demonstrates the importance of positive feedback between different knowledge components or actors in a system. In particular, constituents of a circular process feed into each other forming an autocatalytic cycle leading to improvements all around. ${ }^{36}$ This generates a virtuous circle of knowledge creation that sustains the evolutionary

36 This system of positive feedback has the potential for creating sustained growth in economic development, as highlighted by Levine (2001) for regions of pre-modern Europe. Also see Myrdal (1957) for a theoretical discussion on the circular process of different phenomena leading to a sustained process of economic development. 
process of technological change. However, in chapter 3, I argue that the circular process in knowledge creation is not self-sustaining or self-sufficient by itself i.e., it is not a closed system. The sustainability of a circular process depends on external sources for inputs and may provide its outputs to other actors for further use. In line with the innovation systems literature, a circular process is embedded in a larger network of relationships between heterogeneous economic actors. However, the actors, or knowledge types, in a system connected to each other in a circular process possess relatively greater importance than other actors connected to each other in linear configurations, and act as core components of the system. 


\section{The growth of knowledge and complexity in an evolving network model of technological innovation}

Processes of knowledge production and accumulation are the source of technological development. These decentralised processes, generally involving social interaction among a large group of heterogeneous actors, occur in an auto-catalytic fashion where one novel idea generally leads to another (Metcalfe 2000). Auto-catalytic processes are well studied in the natural sciences and recently formalized in simulation models such as the core-periphery network-evolution model presented in this chapter. I provide a novel interpretation of the model, where the core manifests as a circular process of knowledge flows such as the one discussed in chapter 2, using insights from economics and management studies of innovation. And perform new simulation experiments to study possible transitions from an existing mature technology system to a new one.

This flow of ideas underlying the inventive activity is easily illustrated through a close look at the history of technology. As discussed in section 2.3.2, a radical innovation can be considered as a gradual evolutionary process made up of many incremental steps. For example, the development of the steam engine was a long protracted process spanning decades, involving an incremental accumulation and efforts of a large group of inventors. According to Usher (1954: 68),

"The history of the reciprocating steam engine involves at least five strategic inventions: the atmospheric engine of Newcomen; the low-pressure engine of Watt; the high-pressure engine of Trevithick and Evans; the steam locomotive of Hackworth and Robert Stephenson; the compound engines."1

In addition, as pointed out by Rosenberg (1976), improvements in the interrelated machine tool technology were central to the development of James Watt's steam engine. In the following quote, he illustrates the 'relationship' between the steam engines and co-evolving developments in the machine tool technology.

"The early prototype for this relationship was James Watt's protracted search for a cylinder bored to a minimum degree of accuracy in its diameter. The required degree of precision was eventually attained with the use of John Wilkinson's boring mill, and the commercial practicability of the steam engine really dates from the use of this mill in preparing cylinders" (Rosenberg 1976: 112).

Similarly, according to Musson (1976: 420),

"The average horse-power of all the Boulton and Watt engines built up to 1800 was only 15 h.p., and though the power and efficiency of steam engines were increased in the first half

${ }^{1}$ Here, I do not intend to portray a linear sequence of substitutable inventions that led to the development of a 'perfect' steam engine at the end of the line, but rather demonstrate the flow of diverse ideas as a necessary component of the inventive process. Indeed, some of the inventions mentioned above co-existed and competed with each other for long periods (Frenken and Nuvolari 2004). 
of the nineteenth century, with developments in machine-tools and boiler-making, this growth occurred very gradually..."

In the above examples, note that the incremental steps were built upon knowledge from diverse fields. A synthesis of various steps is generally recognised as a unitary radical innovation ex-post. Radical innovations are "made possible by numerous minor innovations." (Sahal 1981: 20). In addition, inventors generally try several different lines of research for accomplishing a particular technological task. For example, "Edison was not simply investigating one kind of telephone but rather a network of possibilities." (Carlson 2000: 155). The primary idea is that a diverse group of technological practitioners experiment and search for a satisfactory solution in a process that seems akin to trial and error. Consequently, the precise pattern of technological development or knowledge accumulation is not predetermined.

The emergence of a radical innovation, as a combination of incremental innovations, initiates a new era of systemic technological development. This combination becomes the core of a network of interrelated innovations, forming "the basis around which a larger number of further cumulative improvements and complementary inventions" are built (Rosenberg 1982: 59). ${ }^{2}$ However, this systemic accumulation of knowledge is often disrupted by a new radical innovation that initiates technological development along a new path. Synthetic fibres are a prime example of a radical innovation that disrupted the existing knowledge-accumulation pattern in the system of textile technologies. For example, as discussed in \$2.2.2, the existing knowledge of textile spinning was rendered useless for spinning synthetic fibres: a new sophisticated chemical process called melt-spinning had to be developed (Hounshell and Smith 1988a). Numerous other examples can be found to demonstrate the disruptive impact of some radical innovations. The degree of disruption in the existing system-wide knowledge naturally differs from case to case.

This chapter is organized as follows. In \$3.1, I discuss relevant descriptive models of technological evolution. I consider some stylised facts and theoretical constructs that will be represented in the simulation model. In \$3.2, I introduce the model and present a brief description of the model dynamics. Simulation results are discussed in $\$ 3.3$ and simulation experiments are presented in \$3.4. A discussion of the results is presented in the concluding section.

\section{I Models of technological evolution}

Analysis of technological innovation has seen a dramatic upsurge in the last three decades, however few studies have devoted explicit attention to the temporal dynamics of the innovation process (Kenney 2001). One of the first models of technological evolution was presented by Utterback and Abernathy (1975); and Abernathy and Utterback (1978). This product life cycle model was widely accepted and empirically

${ }^{2}$ In the simulation model developed in this chapter, the simplest core manifests as a circular process (or a cycle in graph theoretic terms) among a small number of nodes in the network. 
examined for a significant number of technologies. The life-cycle theory outlines three stages in the evolution of a technology, viewed as a product and its manufacturing process, from its birth to maturity. These stages are based on the similarities observed in five industries (Utterback and Abernathy 1975). The central event in a product's life cycle is the emergence of a 'dominant design' (Utterback 1994; Suarez and Utterback 1995).

The first stage is an era of ferment, characterized by high rates of product innovation and large uncertainty (Utterback and Abernathy 1975; Abernathy and Utterback 1978). Multiple variants of the product are available: both users and producers are experimenting (Utterback, 1994). The variants compete with each other and no standard design has yet been established. The production process is "uncoordinated" and non-standardized during this stage (Abernathy and Clark 1985; Utterback and Abernathy 1975).

In the second stage, an increasing trend towards product standardization is observed. A dominant design eventually emerges as a synthesis of "individual technological innovations introduced independently in prior product variants." (Utterback, 1994: 124). The dominant design is the best compromise solution that satisfies the needs of a wide variety of users. The focus of innovation shifts to the refinement of the dominant design through incremental or 'regular' innovations, which reinforce the dominant design through "improved performance, reliability and lower cost." (Abernathy and Clark 1985).

In the third stage, product and process are both standardized and rates of innovation are slow. Innovations in this stage may be classified as "cost-minimizing" incremental innovations (Utterback and Abernathy, 1975). The mature technology in this stage reaches a limiting point for further technological development. The stage is now set for a radical or "system" innovation leading to a new technological landscape and a probable repetition of the life cycle (Abernathy and Clark, 1985). Such a radical innovation, or a technological discontinuity, generally requires the establishment of a new dominant design followed by a phase of knowledge accumulation on a new technological path (Tushman and Anderson 1986; Anderson and Tushman 1990).

In the product life cycle theory, a technology passes from an initial fluid state to a more specific state (Clark 1985), until the arrival of a technological discontinuity. ${ }^{3}$ Because of the fluidity and ferment in the first stage, the precise nature of the dominant design or the pattern of development in the specific stage is not known a priori.

The life-cycle theory focuses on the evolution of a single technology and fails to emphasise the role of technological interdependence and complementary knowledge in

\footnotetext{
3 At a cognitive level, the transition from the fluid state to the specific state may be viewed as a resolution of "general, nebulous, initial problems" into "specific concrete problems" through "a process of proposing, testing and modifying tacitly understood functional solutions." (Nightingale 1998). A less rational description of the "testing and modifying" process may view it as an experimental trial and error search process based upon certain rules or models.
} 
shaping the dynamics of innovation. In addition, the life cycle perspective is applicable to technologies that are developed in relatively weak appropriability regimes (Murmann 2001). A broader, but in many respects similar, framework for understanding technological development provides insight into the nature of technological 'paradigms' or 'guideposts' that provide the context in which technological problems, and the relevant modes of inquiry, are defined (Sahal 1981; Dosi 1982). A technological paradigm "embodies strong prescriptions on the directions of technical change to pursue and those to neglect." (Dosi 1982: 152). Within the limitations of a technological paradigm, technological development occurs along well-defined 'trajectories' which represent 'normal' problem-solving activities. Here, the search for solutions is restricted to the neighbourhood of what is already known, i.e. search is 'local'. Many incremental innovations take place on a trajectory and the resulting knowledge accumulation occurs along specific directions. Trajectories are inherently 'path-dependent' processes and paradigmatic changes are required to escape a trajectory to initiate a new one. None or very little of the earlier knowledge remains useful after a paradigm change (Dosi 1982). Multiple trajectories within a paradigm generally possess complementarities and may be "more or less powerful" with respect to each other (Dosi 1982: 154). However, it is not known a priori which particular pattern or trajectories will be followed. Thus, the pattern of knowledge accumulation is not predetermined.

Paradigm changes may occur for two reasons. First, technological development along a particular path becomes difficult as technology matures, forcing designers to look for solutions outside the prevailing paradigm (Dosi 1982; Sahal 1985). This search eventually leads to a breakthrough that provides newer avenues for 'progress'. Second, a radical innovation may emerge exogenously, providing a viable alternative to the existing trajectories. However, a paradigm change is generally required to solve problems on the alternative trajectories and to build incremental innovations.

In Dosi's description and in Nelson and Winter's (1977) similar concept of 'natural trajectories', there is an implicit assumption that technologies follow their own internal momentum after a given trajectory has been selected over other competing alternatives. Sociologists and historians have argued against this flavour of technological determinism,

"A persistent pattern of technological change does indeed possess momentum, but never momentum of its own. ... a technological trajectory can be seen as a self-fulfilling prophecy. Persistent patterns of technological change are persistent in part because technologists and other believe they will be persistent." (Mackenzie 1996: 55-56; italics in original).

For purposes of the present model, the important point is that persistent patterns of technological evolution in the form of trajectories are generally accepted. Their persistence may be due to institutional embeddedness (as is the case with a selffulfilling prophecy) or/and embeddedness in a technological system with myriad interdependencies. 
A third related stream of studies has focused on the engineering design process in new product development. Engineering design constitutes a substantial part of the new knowledge required for product innovation. Here the focus of problem-solving is on experimentation as an iterative trial and error process, in which learning through failure and correction plays an important role (Thomke 2001). There is no single optimal solution to a problem and many different design variants/alternatives are tried (Clark 1985). The direction of the design process is not determined by the end-problem, rather the search for complex designs is guided by the "most general heuristics of “interestingness"..." in a sequential step-by-step process (Simon 1981: 186). Each step provides guidance for the next step through identification of errors, or the 'mutation' of weaker elements and the retention of satisfactory ones. This sequence of steps continues until a solution is found: however, it is rarely the optimal solution, in the real world only satisfactory solutions are found (Simon 1981). In product life-cycle terms, the experimental trial and error design process can be treated as the first fluid stage, which culminates with the emergence of a dominant design as a satisfactory synthesis or solution. ${ }^{4}$

Once a satisfactory solution is found, the focus of knowledge creation shifts to the design-improvement and addition of new components/features to the product. This aspect of innovation can be effectively framed in terms of core and periphery components of a complex product system (see Tushman and Murmann 1998; Frenken 2001). With the emergence of a dominant design, architecture of the core stabilises while it is improved further, but newer combinations are tried out among the periphery components (and their connections to the core). Thus, the trial and error search process is now focused on the design of, and architecture of connections between, periphery components. In terms of knowledge creation, the end of the first stage signals the choice of the core design concepts, which set the agenda for the development of 'subsidiary' concepts in a complex product (Clark 1985). For example, in the evolution of the auto-engine, the core concepts would be internal combustion and gasoline, which set the "technical agenda ... for a variety of subsidiary problems and choices. Starting and firing the engine, size and configuration of cylinders, placement of valves and camshafts, and so forth..." (Clark 1985: 243). If an alternative set of core concepts had been chosen, let's say the electric motor instead of the gasoline engine, the subsidiary concepts would be completely different. In this sense, a core may usher in a new technological 'paradigm' whose knowledge base is provided by the constituent knowledge types of the core (which are connected to each other in a circular process in the following model). Such a synthesis of knowledge types, once

\footnotetext{
${ }^{4}$ Many recent studies have highlighted the importance of social, political and market mechanisms in explaining the emergence of dominant designs (see for example Tushman and Rosenkopf 1992; and Tushman and Murmann 1998 for a brief review). In the present model, we do not intend to study the socio-political dynamics behind the selection of one of the competing variants as the dominant design, but to represent the evolutionary trial and error nature of the design-process underlying the development of any of the variants.
} 
successful, shifts the innovation focus to the development of subsidiary or peripheral knowledge around the core, or knowledge accumulation along a well-defined trajectory. Then, in this core-periphery scheme, this structured knowledge accumulation process characterises the second specific stage of the product life-cycle.

At the end of the second stage, the product system is well developed. Small incremental changes continue in the periphery with little impact on the system as a whole. However, with this 'mature' system, further knowledge accumulation along the present trajectory becomes limited and returns to investment reduce dramatically (Sahal 1985). Such a limiting point on the existing path forces designers to look for solutions outside the present paradigm (Dosi 1982). A completely new set of core concepts largely unrelated to the present system may eventually emerge, causing a technological discontinuity. Reverting to Clark's auto-engine example, a discontinuity in knowledge accumulation would appear if fuel cells take over from gasoline engines to become dominant sources of automotive power. In such a scenario, a different set of subsidiary or peripheral concepts will be created in the second stage. In addition, a core-shift can also occur if some peripheral concepts may get transformed to become more central and displace the existing core (Tushman and Murmann 1998). In general, a core shift generally requires a paradigm change, which may make existing knowledge partially or completely useless.

In the next section, I present a simple model of the processes of knowledge creation and accumulation that lie at the root of technological evolution, with the coreperiphery elaboration of product life cycle and other models described in the foregoing providing the necessary structure to the story. Indeed, as Constant (2000: 224) has pointed out, what evolves in technology is "technological knowledge or information." 5 The evolving network model is derived from the complex systems theory, and is a highly simplified representation of complex phenomena. The model was developed by Jain and Krishna (2001) to study biochemical evolution and is based upon the concept of auto-catalytic set studied by Eigen (1971) and Kauffman (1993). The simplicity enhances the analytical tractability of the model and allows us to represent some crucial aspects of innovation that have been largely left out of the realm of formalisation. Silverberg and Verspagen (2004) have expressed the same view in presenting a similar model to explore the dynamics of innovation process while "making very few assumptions about the nature of technology itself."

\footnotetext{
5 Here, the economists', by now widely accepted, distinctions between information and knowledge are not respected. Secondly, knowledge is embodied and any combination of knowledge or knowledge flow has to deal with the tacit component. In our present attempt to develop a simple model to represent the dynamics of knowledge creation, we focus only on the codified component of knowledge. We hope to relax this assumption in a future version of the model by introducing a multiagent scenario and tackling some of the issues surrounding tacit knowledge.
} 


\subsection{Model}

Consider a directed graph with $n$ nodes. Each node in the digraph represents a knowledge type. One may think of a community of practitioners situated around together as a node, engaged in the development of a knowledge type. The links in the digraph indicate the direction of the flow of ideas between different knowledge types. Each link can take any value between 0 (link does not exist) and 1, and the weight of a link represents the relevance of the source knowledge type for the receiving knowledge.

The knowledge types are assumed to exist in a complex technology space or system, and can be more or less susceptible to further development. This susceptibility to improvement or 'innovative dynamism' of a knowledge type depends upon the continuous inflow of new ideas from other knowledge types in the system. ${ }^{6}$ Thus, the flow of ideas acts as the stimulus for innovation at the receiving node. This new knowledge, created through the combination of its own ideas with those received from other nodes, provides the receiving node with the necessary innovative dynamism. Therefore, dynamism at a node depends upon the relevance of the received ideas (linkweights) and the innovative quality of these ideas, i.e. the dynamism at the sender nodes. This dynamism, or more specifically, the relative dynamism of a knowledge type as compared to other types, is the primary variable which evolves on a fast time scale. On this fast time scale, with a fixed link-structure in the graph, nodes receive ideas from other nodes, thereby increasing their innovative dynamism. This process continues until a steady state is reached in the relative dynamism of the population of nodes. More details of the model's evolutionary dynamic are provided later in this section.

On a slow time scale, a selection mechanism operates: one of the least-dynamic knowledge types ceases to be interesting and is dropped from the system, to be replaced exogenously with a new or 'mutated' knowledge type. ${ }^{7}$ This represents the

${ }^{6}$ The contents of the 'transfer' between the knowledge types may be scientific/technical concepts, or ideas about the emergence and the nature of demand or other non-technological knowledge.

7 The node with the lowest relative innovative dynamism is selected out at each time step and variety is introduced into the system in the form of a new node with a fresh set of connections. This evolutionary process based on relative rather than absolute values of dynamism is justifiable even if the modelled network is viewed as a system of innovation. In a SI, some knowledge producing actors or sites may be relatively less innovative than others and get selected out. For example, if the knowledge flow from a producer firm (supplier in production chain terms) to a user firm (buyer) is not sufficiently useful to the latter, it will seek to replace the producer firm with a more innovative one. Here the selection process may be largely viewed as driven by a market for technology (Arora and Gambardella 1994). However, in a SI, non-market selection processes may play a role as well such as pressures of quality/quantity of (scientific or policy) output and accountability acting on academic and government institutions (Malerba 2002). Now if the user firm in our example had the possibility to contract an academic institution (such as a technical university) instead of a producer firm, then selection driven both by market and non-market processes may span an entire innovation system, and in principle, making any relatively less innovative actors more susceptible to selection. This is consistent with evidence from many European regions analysed by Kaufmann and Tödtling 
introduction of exogenous ideas or novelty into the system, and the novel knowledge type, because it will support and be supported by its own set of other knowledge types, gets a new set of incoming and outgoing links.

Under the coupled dynamics on the slow and the fast time scales, the knowledge system evolves, and can create an "auto-catalytic set" of knowledge. That is, a set which is cyclical in the sense that there is a closed flow of ideas between its constituent knowledge types. This continuous cyclical flow of ideas permits innovative dynamism to be sustained at the nodes in the auto-catalytic set. I interpret this set to represent the core concepts underlying a complex product system described in section 2.0. The sustained dynamism of the core leads to accumulation of incremental innovations in the system, as other knowledge types build upon the ideas generated at the core.

\subsubsection{Model dynamics}

The number of knowledge types in the system, $n$, remains fixed during a simulation run. The innovative dynamism of each knowledge type is initialised with a random value between 0 and 1.8 These values are then normalised such that,

$$
\sum_{i=1}^{n} v_{i}=1
$$

Note that $v_{i}$ after normalisation represents the relative value of the dynamism at a node. A connection probability $(p)$ determines if a link $\left(l_{i j}\right)$ from node $j$ to $i$ is nonzero. The value of $p$ is kept small to portray that, at any point in time, relevant ideas can flow between a small number of knowledge types. ${ }^{9}$ The weights of the non-zero links, $w_{i j}$, are randomly chosen between 0.01 and 1.00. A nonzero $w_{i j}$ represents a non-trivial flow of ideas from knowledge type $j$ to type $i$. Here, self-links are not allowed, i.e. $w_{i j}=0$ if $\mathrm{j}=\mathrm{i}$, but bi-directional links are allowed, in which $i$ supports $j$ and $j$ supports $i$. This procedure generates a sparsely connected digraph of size $n$.

With the above initialisation and keeping the graph structure fixed, the system is allowed to relax to a stable state on the fast time scale. The stable state is given by the attractor of the following dynamical system of equations,

$$
\dot{v}_{i}=\sum_{i=1}^{n} w_{i j} v_{j}-v_{i} \sum_{j, k=1}^{n} w_{k j} v_{j}
$$

(2001), who argue that university-industry exchange may be relatively more important than userproducer interactions among firms and thus technology policy should stimulating the former to encourage diversity in a SI.

8 As will be clear in the following, simulation results do not depend upon the initial conditions.

${ }^{9}$ Here, $p$ may be viewed as a simple proxy for the nature of the intellectual property rights (IPR) regime in the system, which as highlighted by Murmann (2001) affects the nature of life-cyclic evolution of technologies. A small value of $p$ (restricted flow of ideas) suggests a highly proprietary regime whereas a very large $p$ may indicate an open IPR regime where a free flow of ideas (between the communities of practitioners around each node) is possible. 
where $\dot{v}_{i}$ represents the rate of change of the innovative dynamism of a knowledge type $i$ and $w_{i j}$ is the weight of the link to node $i$ from $j$. The first term on the right hand side of equation (3.2) shows that the rate of change of relative dynamism of type ' $i$ ' is a function of the weight of all links it receives and the relative dynamism values of the source knowledge types. The second term, of a smaller magnitude as it is a square of normalised values, is necessary to preserve the normalisation of $v_{i}$ on the fast time scale. To summarize, on the fast time scale: given the set of knowledge types, the system evolves towards a stable state in which the direction (the link structure) and relevance (link-weights) of knowledge flows between the different types remains fixed. The set of coupled variables evolving on this time scale is the relative dynamism values of the knowledge types.

The set of coupled differential equations (3.2) is solved numerically using the Runge-Kutta method to find the attractor. After the system has settled to an attractor, selection takes place in the first slow time period: one of the nodes from the set of lowest relative dynamism is selected out of the system, along with all its links. This knowledge type is replaced with a new or 'mutated' type with a new set of incoming and outgoing links. This introduction of novelty, or variety, is largely a memory-less process because the probability that a new mutated type retains any links of the node it has replaced is infinitesimally small. ${ }^{10}$ The new type is assigned a very low $v_{i}$ and its new outgoing and incoming links are nonzero with the same probability $p$. The weights of the nonzero links to and from the new node are once again randomly assigned between 0.01 and 1.00. All other types are perturbed slightly from their steady state values and the system is normalised according to equation (3.1). Note that the new links change the graph structure. Keeping the new updated graph structure fixed, the system is solved using equation (3.2) on the fast time scale to reach the new attractor. Now, in the second period on the slow time scale, the retention, selection, and variation processes are repeated to set up the system for solution on the fast time scale once again. Most simulations presented in this chapter are run for 2000-5000 slow time-periods. The computer program for the model is written in $\mathrm{C}++$. The code is available on request.

To summarize, during a slow time period, given the types of knowledge available and the link-structure between them, the innovative dynamism in the system evolves on the fast time scale (until a stable state is reached). Each node responds to the ideas it receives from other nodes, repeatedly improves the quality of its knowledge, and creates new knowledge by combining the received-ideas with its own knowledge. However, in this process, it can happen that some knowledge types are not as well supported as others and their dynamism is relatively weakened. Consequently, these types are of little use to the knowledge types they have been supporting. On the slow

10 Relaxing this assumption, by allowing the mutated type to retain part of the links from the previous time period and assign some new links based upon a search heuristic, may yield a more realistic picture of variety creation in a technological or innovation system. 
time scale, they are removed by selection, and new types of knowledge are introduced, again providing support to other types.

\subsection{Simulation results}

In this section, I present simulation results for different values of $p$. The size of the system $(n)$ is kept constant at 50 in all runs. ${ }^{11}$ The number of knowledge types mutated in each slow time-period (simply referred to as a time-period in the remainder of this chapter) is 1 . In this section, I provide a brief description of system behaviour observed in the simulations, followed by an investigation of the underlying mechanisms.

The number of nodes with nonzero relative dynamism in the attractor of each time period $\left(n_{1}\right)$ is plotted in Figure 3.1 for three simulation runs with $p=0.0025,0.005$ and 0.0075 . In each case, the behaviour of the system can be divided into three distinct phases before a collapse in the knowledge system is observed. The first phase represents the experimental trial and error phase when many different knowledge-flow configurations are tried. The new knowledge created is reflected in nonzero relative dynamism values in the attractor for a small number of types. The chance appearance of a 'cycle' of nodes in the graph signals the end of the first phase. ${ }^{12}$ The cycle, because of its "auto-catalytic" nature, becomes sustainable in the long run and I view it as the core of the technology system. In this second phase, the introduction of novelty causes innovative dynamism to percolate through the system as new, mutated nodes receive links from the core. In the third phase, the system is 'complete' in the sense that approximately all knowledge types possess high values of $v_{i}$. Here I have a relatively 'mature' system in which all its parts are innovative to roughly the same degree, so tensions are created by some part racing ahead of the rest of the system, or some part falling way behind. This phase is brought to an end by a discontinuity due to a core-shift in the system. Details of system behaviour and the underlying mechanisms are discussed below.

11 A more realistic representation should allow the number of types to increase as the system evolves to greater complexity. However, as we will show later, the number of knowledge types with high relative dynamism rises until the system is fully constructed.

12 Jain and Krishna (2002) define a 'cycle' of n nodes as a sub-graph with $n$ nodes which contains exactly $n$ links in a circular configuration. In other words, a cycle is a directed sub-graph, each of whose nodes can be reached from any other node in the same sub-graph, i.e. it contains a closed path covering all its nodes. In a closed path, the first and the last nodes are the same. 


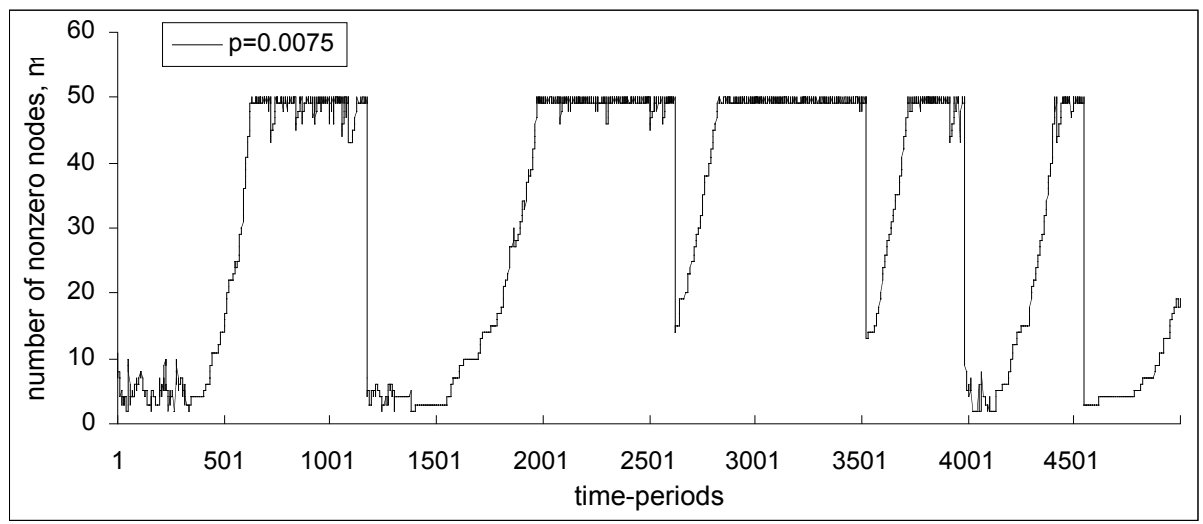

3.1 a)

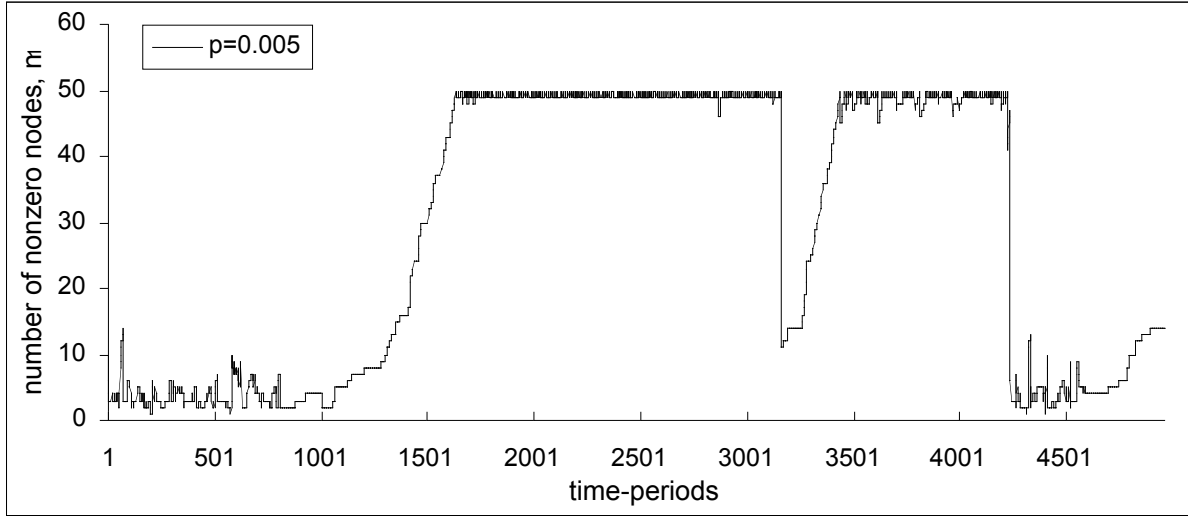

$3.1 \mathrm{~b})$

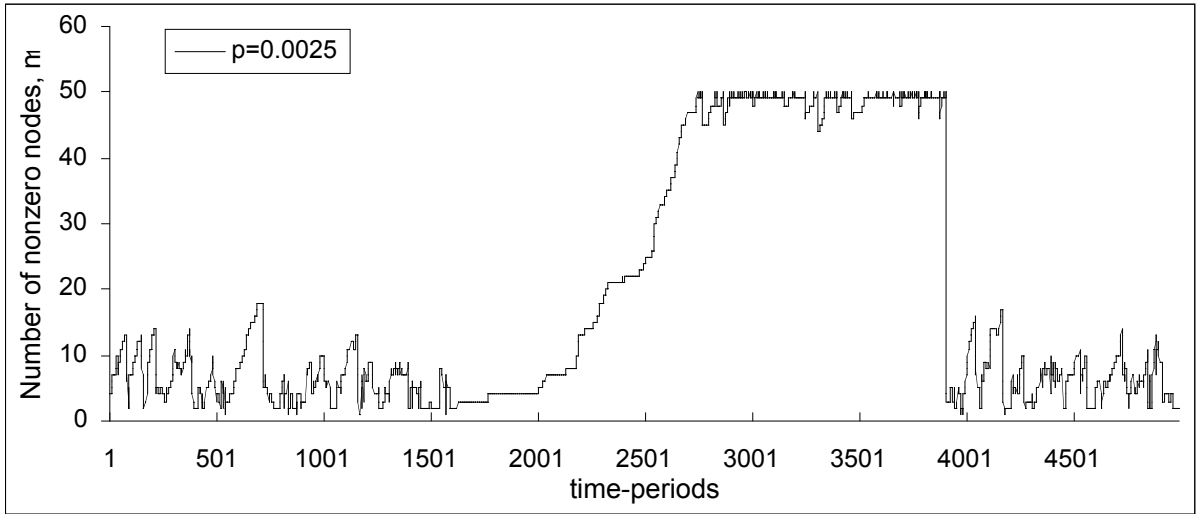

$3.1 \mathrm{c})$

Figure 3.1. Simulation results showing the three phases in the evolution of the technology system. The first experimentation phase, followed by the accumulation phase, and the 'mature' phase, before the arrival of a discontinuity. Note that the duration of the first phase increases as the connection probability, $\mathrm{p}$, is decreased from 0.0075 to 0.0025 . 
The first phase in Figure 3.1 represents the knowledge creation process through experimentation: various knowledge-flow configurations are being tried. In this phase, flows between knowledge types are structured in multiple disjoint (linear or tree-like) paths. The node at the end of a path receives ideas from the upstream 'supporting' nodes in that path, attaining higher values of relative dynamism as compared to other nodes in that path. ${ }^{13}$ However, the selection and variation processes randomly replace one of the supporting nodes that has zero relative-dynamism in the attractor, and thereby disrupt the existing paths. As a result, the nodes can typically sustain their high dynamism values only for a few time periods. ${ }^{14}$

The first phase lasts until a cyclic arrangement of nodes appears. The time duration of this phase depends upon how freely ideas can flow in the system, i.e. the connection probability, $p$. As shown in Figure 3.1, the average duration of the first experimentation phase reduces as $p$ is increased. ${ }^{15}$

The second phase is triggered by the formation of an auto-catalytic set (ACS) as a cyclic structure between different knowledge types. ${ }^{16}$ Unlike the mostly linear structures formed in the first phase, a cycle is self-sustaining due to the mutuallyreinforcing flow of ideas among its constituent nodes. Therefore, all nodes in the cycle possess high values of relative dynamism in the attractor and are not targeted by selection in this phase. The knowledge types of a cycle may be considered complementary, in the sense that an improvement in one improves the dynamism of the others. The self-sustaining nature of the cycle makes it the core around which the remaining system is gradually constructed. In order to sustain their dynamism, the core knowledge types do not need inflow of new innovative ideas from other types. But should an inflow of new ideas exist, it would of course make the core more dynamic.

In the second phase, the focus of experimentation and design-search processes shifts to the knowledge types outside the core. The selection and variation processes create a gradual systemic creation of knowledge as new nodes get incoming links (or a chain of links) originating in a core node. ${ }^{17}$ Here, the ideas generated at the core in

13 Thus, in each attractor during this first phase, the nodes at the end of the longest paths are the only ones possessing nonzero values of relative dynamism as they receive ideas from the largest number of upstream nodes (see Figure 3.2(b)).

14 Therefore, sustainability of the relative dynamism at a node depends upon the upstream supporting nodes possessing nonzero $v_{i}$ themselves. Such a scenario can only arise if a cyclic structure is formed between the nodes.

${ }^{15}$ By average duration, we mean the average over a large number of simulation runs. The randomness in the structure of the model does not ordain a predetermined duration of the experimentation phase in each run.

16 "An autocatalytic set (ACS) is a subgraph, each of whose nodes has at least one incoming link from a node belonging to the same subgraph. ... An ACS must contain a closed path." Jain and Krishna 2002: 7). The simplest closed path is a cycle of nodes.

17 Since only one knowledge type is mutated in a time-period, a maximum of one node can receive a link from the core at a time. But the addition of a single new node can provide a bridge from the core to an already existing chain of nodes, and thus indirectly create support for many nodes. 
each time period may be passed to the new or 'mutated' type, where they provide stimulus for the creation of subsidiary or peripheral knowledge. This new knowledge is reflected in the high relative dynamism values of the new types. Gradually, relative dynamism percolates to all the outer nodes in the graph as they get incoming paths from the core: a 'complete' system is realized at the end of the second phase. Note that without the core in the first phase, the selection and variation processes produced no accumulation in the system.

In a 'complete' system, all periphery nodes lie on paths originating in the core, as shown in Figure 3.2(a). The relative dynamism at a node depends upon the number of links it receives, the weight of these links and the relative dynamism values at the sender-nodes. Here the core nodes are transmitting ideas continuously without receiving any new ideas from one of the other nodes. ${ }^{18}$ Consequently, with certain linkstructures in a 'complete' system, the core nodes may become less dynamic than the periphery nodes that receive a continuous inflow of ideas. Eventually, one of the core nodes joins the set of nodes with the lowest value of relative dynamism. The replacement of this core node in a subsequent time period causes a discontinuity or collapse in the existing knowledge system. ${ }^{19}$

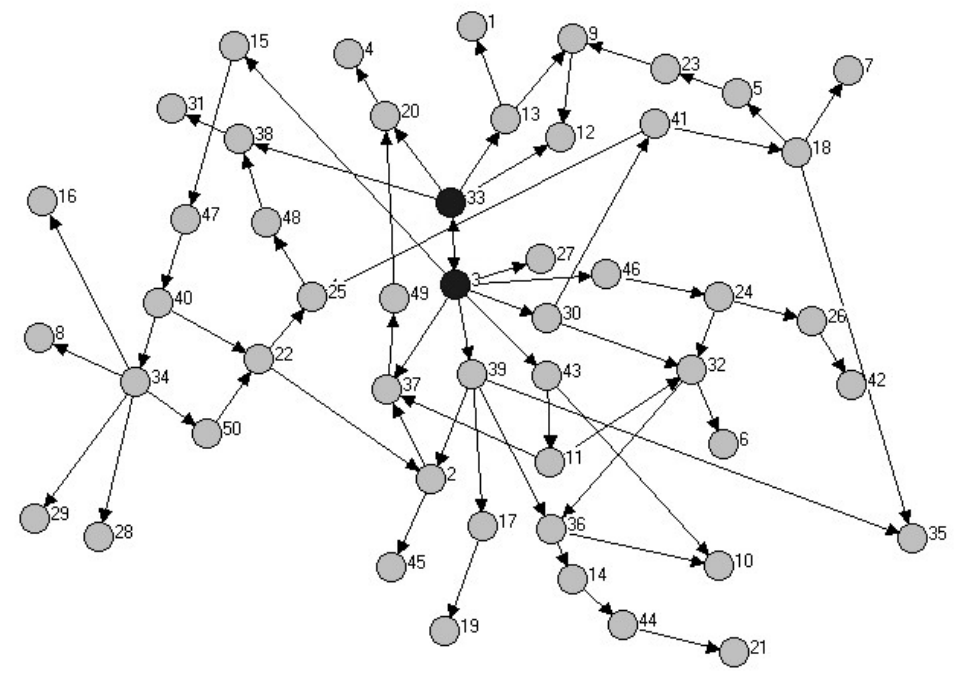

Figure 3.2 (a). An example of the graph structure in fully constructed system. The core is the cycle between nodes 3 and 33 .

18 In a 'complete' system, if a new node feeds into a core node but receives no incoming path (chain of links) from the core, it will fall in relative dynamism eventually and get selected out. On the other hand, if this new node also receives an incoming path from the core, it becomes a part of the core: this enbancement of the core is described below and depicted in Figure 3.3.

19 A discontinuity or collapse is assumed to occur if the number of types with nonzero $v_{i}$ in the attractor of time-period $t, n_{1}(t)$, is less than $n_{1}(t-1) / 2$. 


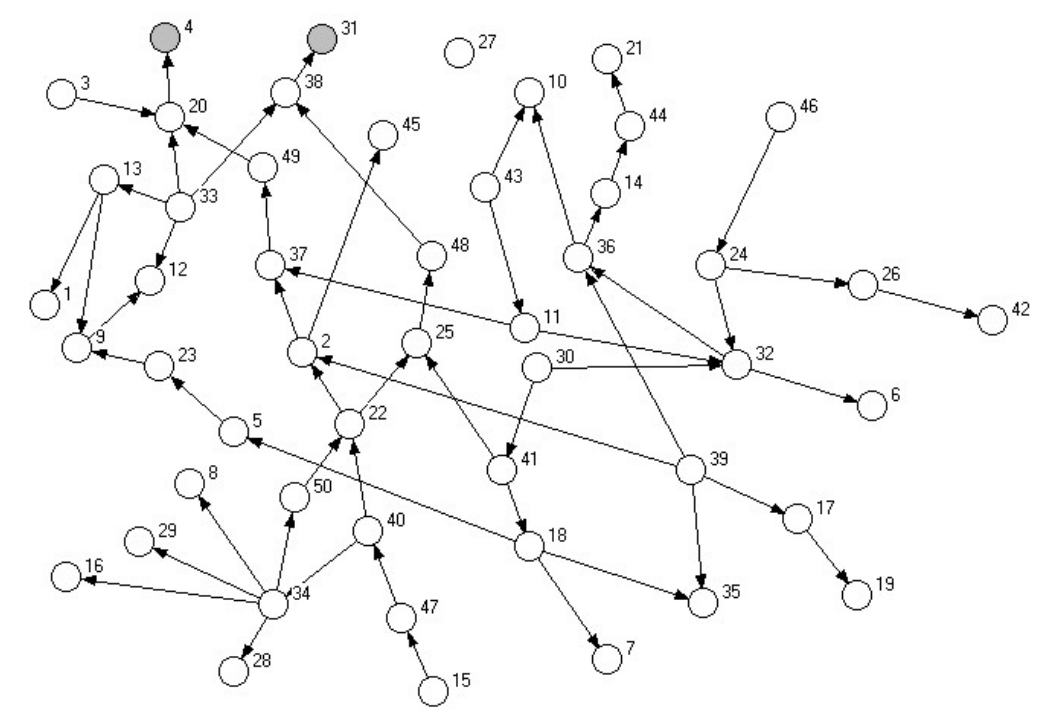

Figure 3.2 (b). The same system as in Fig. 3(a) after a discontinuity: mutation of element 3. Only two nodes (4 and 31) possess nonzero viability as they lie at the end of the two longest paths originating in node 15 .

A simple cyclic arrangement between nodes (represented as a circle) is the minimum condition for the formation of the core. Here a core of $\mathrm{n}$ nodes will be minimally connected, with $n$ links. Other possible cores can have additional internal links between the constituent nodes of the core, making a denser sub-graph, and each additional link makes the positive feedback within the core stronger. ${ }^{20}$ The internal link-structure of a cycle, once formed, cannot be changed because all its members have high relative dynamism values, and do not get targeted by selection until a discontinuity occurs. However, an existing core can be increased in size and link density if a periphery nodes gets an incoming link from, and sends an outgoing link to, the core. An example of such a core enhancement is shown in Figures 3.3.

${ }^{20}$ For digraphs with non-negative links of the type discussed here, the strength of the core is determined by the Perron-Frobenius eigenvalue (PFE) of the adjacency matrix of the graph. The related eigenvector gives the relative dynamism values of the knowledge types in the attractor. The calculation of this eigenvalue and the eigenvector is a cumbersome task if matrix elements (linkweights) are allowed to take values between 0 and 1, as is the case here. Jain and Krishna (2002) allow link-weights to be either 0 or 1 , and calculate the PFE and the related eigenvector to solve the dynamical system. 


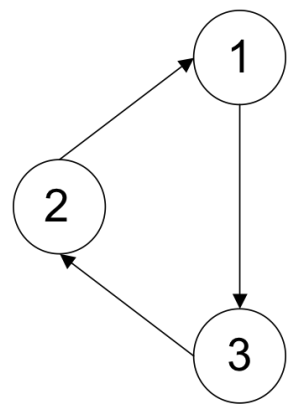

Figure 3.3 (a) A cycle of 3 nodes as the core before enhancement.

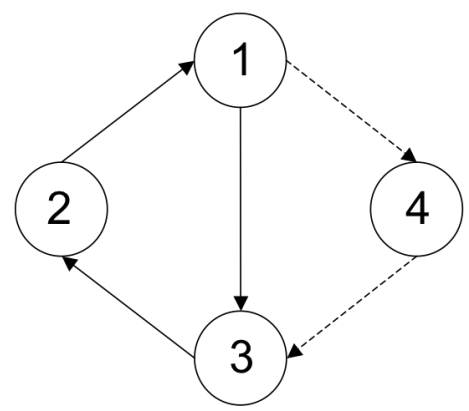

Figure 3.3 (b) The 3-cycle core is enhanced to a core of 4 nodes (with an additional internal link) by the addition of node 4 . The dashed lines represent the new links.

The rate of growth (speed with which additional nodes get connected to the core) in the system depends upon the connection probability, $p$. The larger the value of $p$, the faster the system is 'constructed' (compare Figures 3.1(a), (b) and (c)). An analytical explanation of this result is given by Jain and Krishna (2002). Let us assume that the number of types in the core of the system is $n_{1}$. Then the probability that a periphery node gets a link from one of the core nodes in a time period is $p n_{1}$. On average, the number of nonzero types in the system will grow by,

$$
\Delta n_{1}=p n_{1} \Delta t
$$

where $\Delta t$ is the number of (slow) time-periods or graph updates. If $t_{a}$ is the time of arrival of the core and $n_{1}\left(t_{a}\right)$ is the size of this core, then at time $t$,

$$
n_{1}(t)=n_{1}\left(t_{a}\right) \exp \left\{\left(t-t_{a}\right) * p\right\}
$$

where $n_{1}(t)$ is the number of nonzero types in the system (or size of the system) at time $t$. The system is assumed to be 'complete' when $n_{1}(t)$ is approximately equal to $n$ minus the number of nodes mutated in a time period (equal to 1). Thus, the time taken for the construction of the 'complete' system from the time of arrival of the core is given by $(1 / p) \ln \left\{(n-1) / n_{1}\left(t_{a}\right)\right\}$ (Jain and Krishna 2002).

The third phase begins when the system is 'complete'. Knowledge accumulation slows down and a simple cyclic core eventually gets weakened relatively as described above. In this 'mature' system, changes through selection/variation generally have little impact on system behaviour if a periphery type is mutated. In contrast, if a core 
knowledge type is mutated, a discontinuity in the knowledge accumulation regime is observed, and a core shift is observed. ${ }^{21}$ However, if the core is powerful enough (in terms of high link-density and link-weights within the core), its nodes are not targeted by selection due to their high $v$, and the system remains 'complete' for a long time. Simulation experiments investigating the robustness of the system with cores of different strengths are discussed in the next section.

A discontinuity in the knowledge system can have three different underlying causal mechanisms. First, a discontinuity occurs if, in a 'complete' system, a core-node is selected out due to its low relative dynamism value. The intuition behind this type of a discontinuity is that the core knowledge types reach a point where further improvement becomes very difficult (the limiting point along a trajectory discussed in section 2.0). Alternatively, the selection process here may be viewed as an exogenous shock to the system (which collapses due to the weak core). Subsequently, technological practitioners search for a solution from outside their present knowledge set, which requires a new experimentation phase. This process is depicted in the first collapse shown in Figure 3.1(a). Formation of a new core signals the end of the experimental trial and error and a new phase of knowledge accumulation is initiated.

Second, a core-shift may occur in the system if a newly introduced node forms a cyclic structure with some other periphery nodes. If this new structure is more powerful than the existing core, it becomes the dominant core causing an immediate collapse of the older accumulation regime. Such a discontinuity is not followed by an experimentation phase as the new core is already constructed in the older periphery. The amount of disruption caused by a discontinuity varies from case to case (compare the different collapses shown in Figure 3.1).

The third type of discontinuity starts in a similar fashion as the second type with the arrival of a peripheral cyclic structure. However in this case, the new structure is less powerful than the existing core and does not cause an immediate collapse. This new cycle remains in the periphery until it is enhanced through the addition of more nodes (and links) in subsequent time periods, or until the existing core is weakened due to the removal of one of its nodes.

The third phase (before the discontinuity) is characterised by self-organisation of the system to a state of high complexity and interdependence, measured by the average number of links per node and the average weight over all links. Emergence of the core is the central event that ushers in this self-organisation. This is shown in Figure 3.4 for results from the same run as in Figure 3.1(b). As shown in Figure 3.4(a), after the formation of the ACS, the total number of links per node rises dramatically as the periphery nodes get connected to the core and are retained (along with their links) in the system. Note that the number of links with weights higher than the average value $(=0.50)$ increases more rapidly than the number of below average-strength links. At

${ }^{21}$ A core-shift can also occur if a stronger cyclic structure (than the existing core) is formed in the periphery. This new core then acts as the dominant core. The different types of core-shifts are discussed later in this section. 
some point in the third phase, the number of below average-weight links starts decreasing but the above average links continue to rise. This self-organization occurs because new nodes with higher link-weights have a greater chance of retention in the system, due to their higher relative dynamism values in the attractor according to equation (3.2). As a result, the average weight of the links in the system increases in the third phase, as shown in Figure 3.4(b). The self-organizing behaviour shows that knowledge accumulation and growth continue in the 'mature' system.

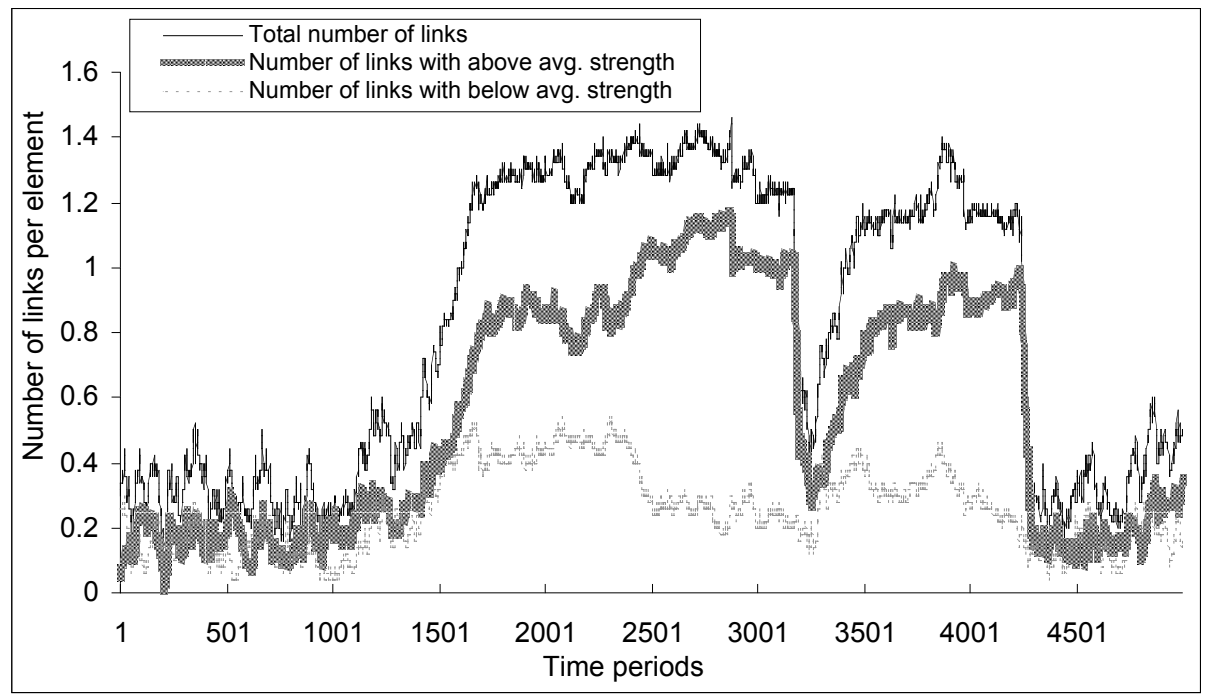

3.4 (a) Number of links per node (Total number of links in the graph/total number of nodes).

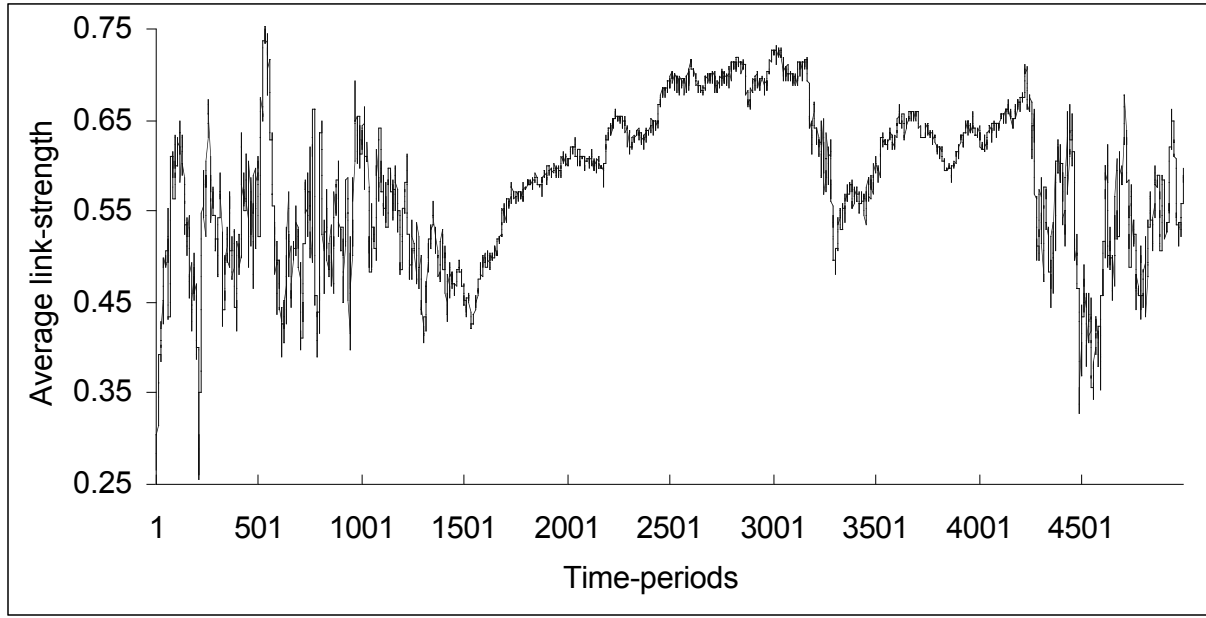

(b) Average weight over all links in the system.

Figure 3.4. Self-organization in the system. Results plotted here are for the same run as the one shown in Figure 3.1(a), $\mathrm{n}=50$. 
In the model, we observe a spontaneous emergence of order and structure in system behaviour driven by a highly simplified evolutionary dynamic. Throughout a simulation run, retention, selection and variation mechanisms follow the same rule but the system behaves differently over time, as in the three phases. In the first phase, the introduction of novelty generates innovative dynamism in some knowledge types, which cannot be sustained for a long time and no knowledge-accumulation is observed. However, in the second phase, due to the chance formation of a selfsustaining core, the introduction of new ideas spurred by the dynamism of the core causes a systemic accumulation in the stock of knowledge. In the third phase, the robustness of the 'mature' system against exogenous shocks (core-shifts), and its ability to assimilate new ideas, depends upon the dynamic strength of the core. Here, new ideas that are more relevant (higher link-weights) to the existing system have a greater chance of getting successfully assimilated, see the self-organization discussion above. On the other hand, system behaviour in the third phase has important implications for forcing a shift from an existing knowledge regime: introducing a group of mutually reinforcing concepts (as a cycle) into the 'mature' system has a greater possibility, than the introduction of single technologies, of producing the desired regime shift. In the following section, I briefly investigate some regime-shift scenarios through the introduction of new cyclic structures into the periphery of a 'complete' system. In addition, the robustness of a system as a function of its core-strength is explored.

\subsection{Simulation Experiments: Different Core Structures}

In this section, results from simulation experiments with different core configurations are presented. First, I investigate the robustness of the system with three different link-structures within the core. This is followed by a brief discussion on the behaviour of a 'mature' or 'complete' system to the entry of a new competing core. The strength of a core is a function of the number of internal links between the corenodes (link density) and the weights of these links. ${ }^{22}$ These simulations were started with a fully constructed system (with a core of known structure), and incoming links to the core were not allowed to prevent core enhancements. The weight of all links in the existing core and the new competing structure is 0.5. Experiments examining the impact of an increase in the number of knowledge types mutated in each time-period are also discussed.

\subsubsection{Core-strength}

A very 'strong' existing core is able to exclude all new cyclic combinations emerging in the periphery of the system. ${ }^{23}$ The additional internal links within a core

\footnotetext{
22 In the following, I study the impact of link-density in the core only. The link weights in the core are kept constant in all experiments. An investigation of core-strength as a function of its internal linkweights is left for a future study.

23 This core-strength may be interpreted in terms of the 'power' of a particular technological trajectory. Using an analogy from epistemology, Dosi (1982: 154) argues that "a trajectory is more
} 
allow direct flow of ideas between nodes that are otherwise indirectly linked. For example, in Figure 3.3(b), due to the additional link, node 1 gets direct 'support' from node 3 instead of an indirect one through node 4. Here, I compare the impact of a cyclic core structure, a complete core (all possible connections between the member nodes) and a cyclic core with two extra internal links. The number of time-periods with a fully constructed system before a discontinuity is used to measure the robustness of the system. The results are plotted in Figure 3.5 for a core of size 4. Similar results were obtained with other core sizes. As shown in the figure, the robustness of the system is highest for a complete core where no discontinuity occurs in the system. The system with two additional internal links in its core is more robust than that with a simple cycle.

The reasons for highly robust behaviour of the system with a complete core are twofold. First, the large number of positive feedback loops in the core result in higher relative dynamism values for all core-nodes in the attractor. Second, the link-density in the core enhances the ability of the system to ward off potential core-shifts to other cyclic structures formed in the periphery. These peripheral structures, formed due to the selection and variation processes, are mostly cyclic due to the small value of $p$.

In the cyclic core with two additional links, one or two core-nodes which receive an additional link possess higher relative dynamism values (for example, node 1 for the core shown in Figure 3.5, which receives two incoming links). The nodes that do not receive additional links can become relatively less dynamic than the periphery nodes. ${ }^{24}$ One of these core nodes is eventually selected out, which reduces the link-density (and size) of the core, and may cause a partial collapse. Note that even after this removal, a cyclic configuration can be retained among the remaining core nodes. For example, as shown in Figure 3.6, if the core node 3 is replaced, a cyclic structure between nodes 1 , 2 , and 4 remains in the system: periphery nodes on paths originating on these nodes sustain their dynamism. However, the system with a simple cyclic core is more susceptible to a future discontinuity. Finally, here a core-shift may also occur if a simple cyclic structure with very high link-weights is formed in the periphery. ${ }^{25}$

powerful the bigger the set of technologies which it excludes." In addition, it is difficult to switch from an existing "very powerful" trajectory to a new one.

${ }^{24}$ Here the nodes with no additional links get relatively weakened in the same way as the nodes in a simple cycle. However, these nodes get incoming links from other, stronger core nodes (that receive more than one link), which increases their dynamism as compared to the nodes in a simple cycle.

25 Note that core-strength depends upon the link-density and the link-weights within a core. Therefore, a simple cyclic structure with high link weights may be stronger than a core with additional internal links but relatively low link-weights. 


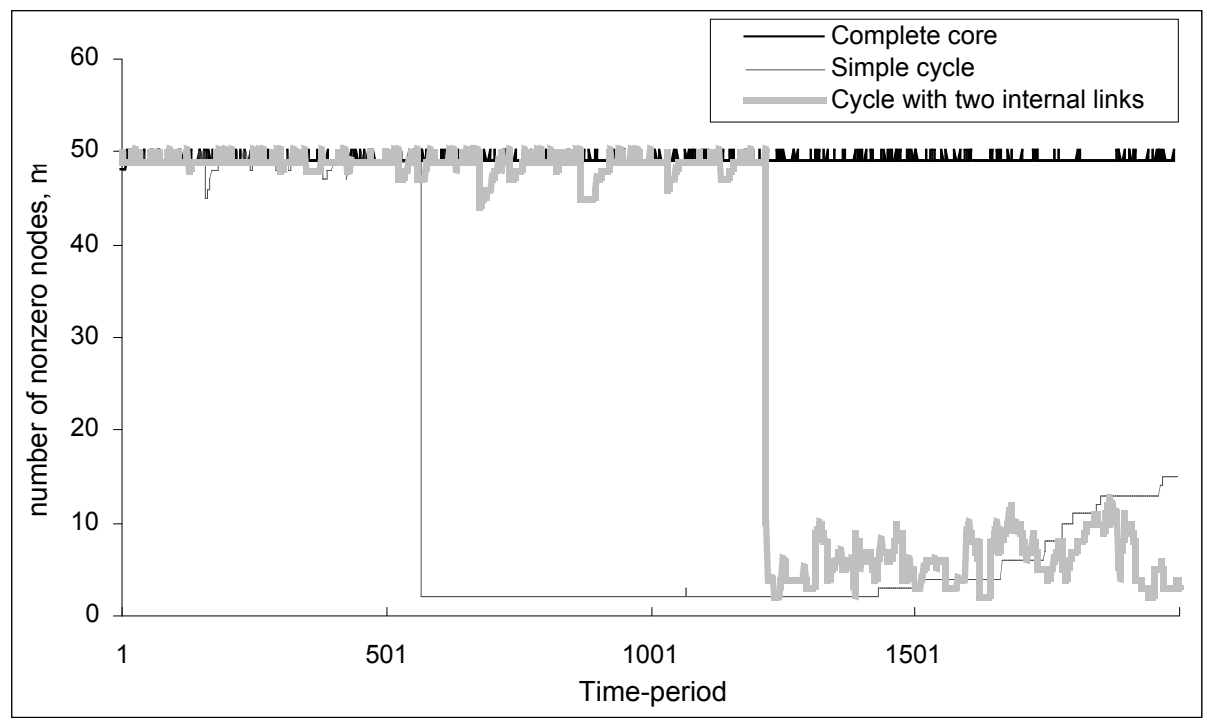

Figure 3.5. System robustness with a complete core, a simple cyclic core, and a cyclic core with two additional internal links. All three cores are of size 4.

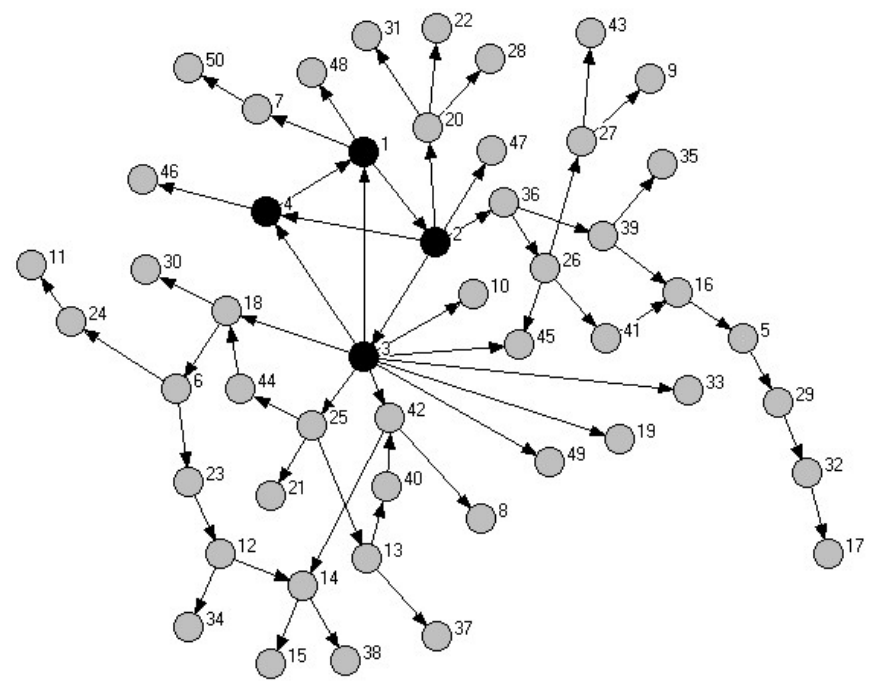

Figure 3.6. A system constructed around a 4 node core with two additional internal links. If node 3 is selection out, a simple cyclic core between nodes 1,2 and 4 remains in the system and the periphery nodes connected to these nodes will sustain their innovative dynamism.

A second set of simulations was performed to investigate the impact of introducing a new cyclic structure into the periphery of a 'complete' system. As the link-weights in the new structure and the existing core are kept the same here, core- 
strength is only a function of the link density. ${ }^{26}$ As shown in Figure 3.7(a), if the new structure has a higher link-density than the existing core, a core-shift takes place forcing a shift in the existing knowledge regime and the new core becomes the dominant core. The new core here is stronger than the existing one. The higher strength simply implies that a greater number of positive feedbacks exist among the constituent knowledge elements of the new core as compared to the older one. This denser structure of positive feedback loops creates the advantage that causes the 'escape' from the lock-in to the existing core and system. On the other hand, if the new structure is weaker than the existing dominant core, it is assimilated into the system periphery and a core-shift does not occur (see Figure 3.7(b)). In this case, the structure of positive feedback loops is denser in the existing core creating advantage for the latter.

In a third scenario, where the new and existing cores have the same link-density, a core-shift occurs only if the new structure is downstream from the existing one; ${ }^{27}$ results from this simulation are shown in Figure 3.7(c). The (new) downstream core is then the dominant core. This result on the downstream core may be interpreted as follows. The structure of positive feedbacks in the two cores are equally dense in this case, so there is no clear winner around which a new system can be built to replace the existing one. Any of the above three scenarios of positive feedback structures in a core set of knowledge types can arise in the history of technology (see Chapter 2 for some historical examples of circular structures among knowledge types).

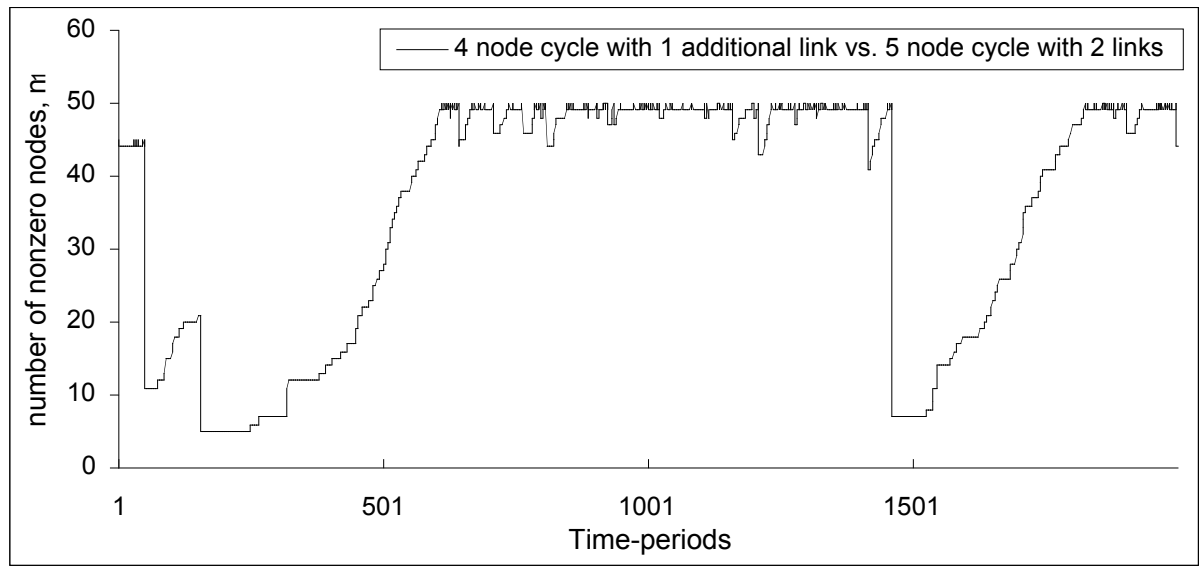

3.7 a) Discontinuity (in period 50) in a system with a 4 node cyclic core with one internal link through introduction of a stronger, 5 node cycle with two additional links.

${ }^{26}$ Core strength is then simply given by the density of internal links in the core, $d_{i L}=\frac{{ }^{n} L^{-n}}{n(n-1)-n}$.

27 Downstream implies that at least one of the nodes in the new structure gets an incoming path originating in the existing core; and there is no path from the new to the existing core (Jain and Krishna 2002). 


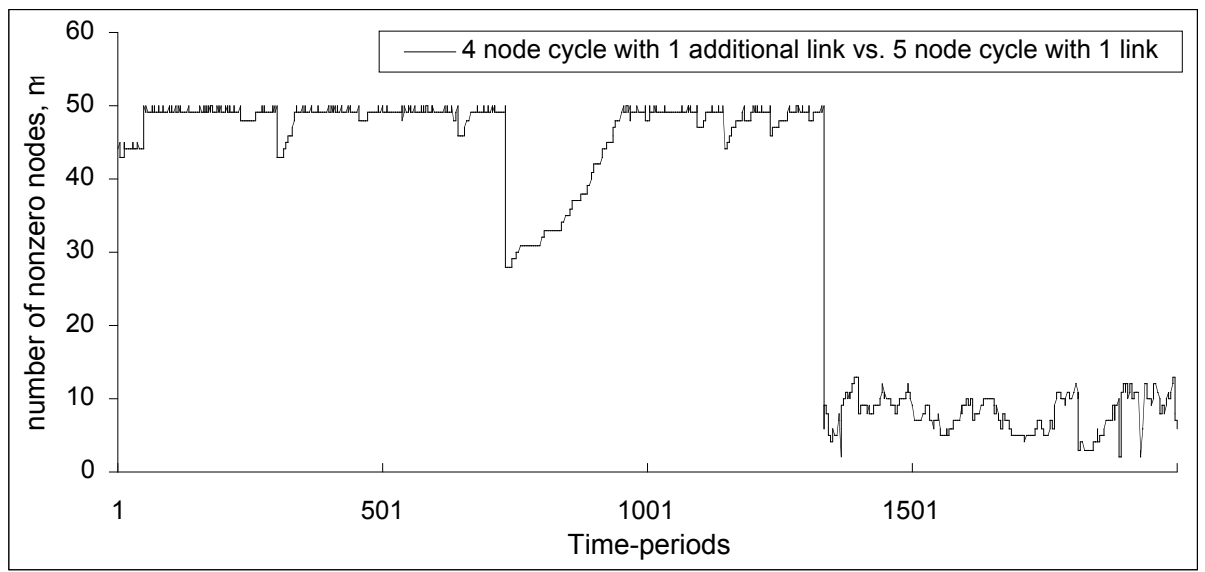

3.7 b) The new cyclic structure introduced (in time-period 50) is assimilated into a constructed system with a stronger core: the new 5 node cycle has lower link-density.

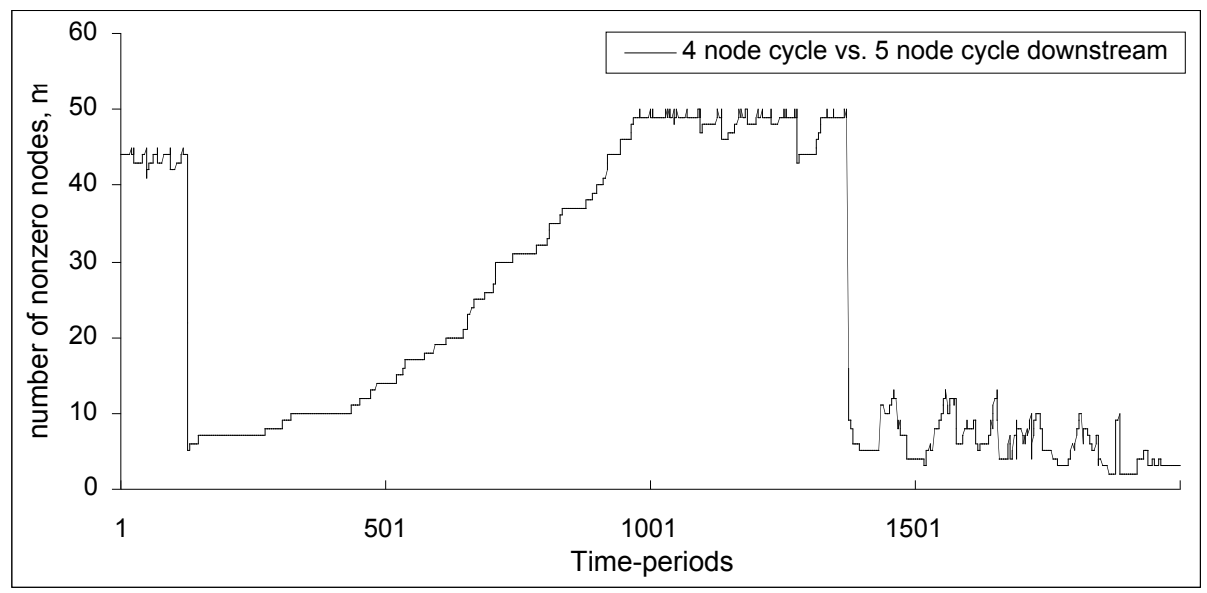

3.7 c) Discontinuity (in period 150) caused by the introduction of a cycle of equal strength that is downstream from the existing core.

Figure 3.7. Introduction of new cyclic structures into a fully constructed system. The size of the system before the introduction of new elements is equal to 45 .

\subsubsection{Number of knowledge types mutated in one time-period}

The impact of the number of nodes 'mutated' in a time-period (ns) is shown in Figure 3.8. An increase in $n s$ makes system behaviour more unpredictable and less robust to exogenous shocks: on average, the number of discontinuities increases; partial and complete collapses in the knowledge accumulation regimes become more frequent (compare the system behaviour in Figures 3.8(a), (b), (c)). This happens because the probability of 'mutation' of a relatively weakened core node in a 'mature' system increases. In addition, mutation of a larger number of knowledge types increases the probability of formation of more powerful cyclic structures in the periphery. The greater probability of formation of cycles also reduces the duration of 
the first experimentation phase. This duration reduces dramatically as $n s$ is increased from 1 to 3 . A less sharp difference is observed between runs with $n s=3$ and $n s=5$.

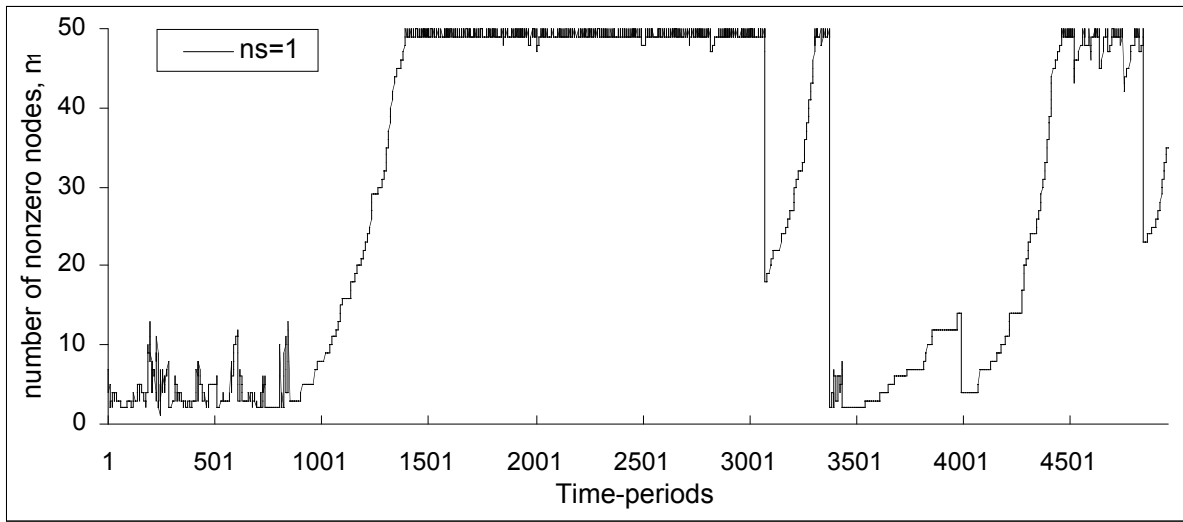

3.8 a)

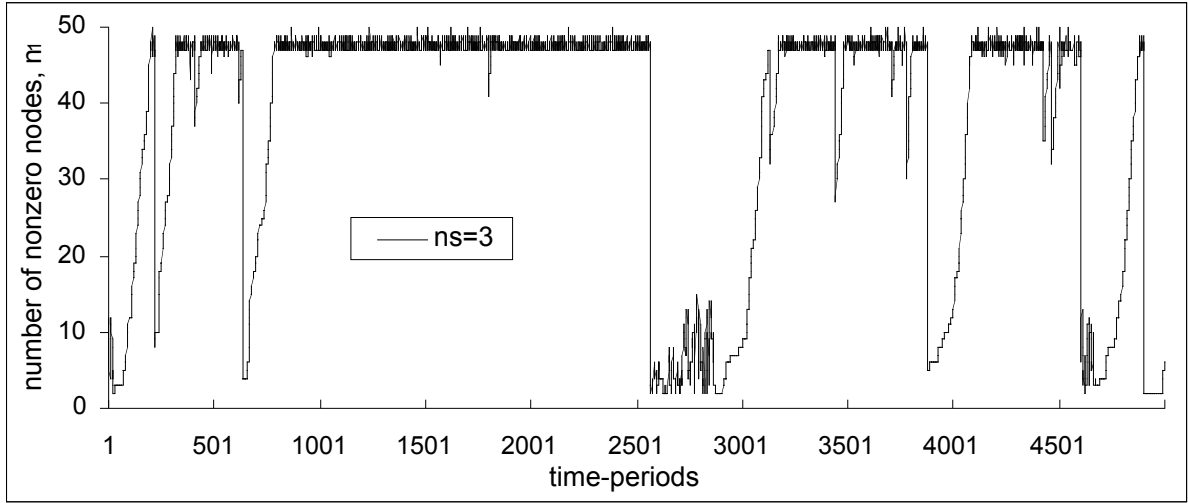

$3.8 \mathrm{~b})$

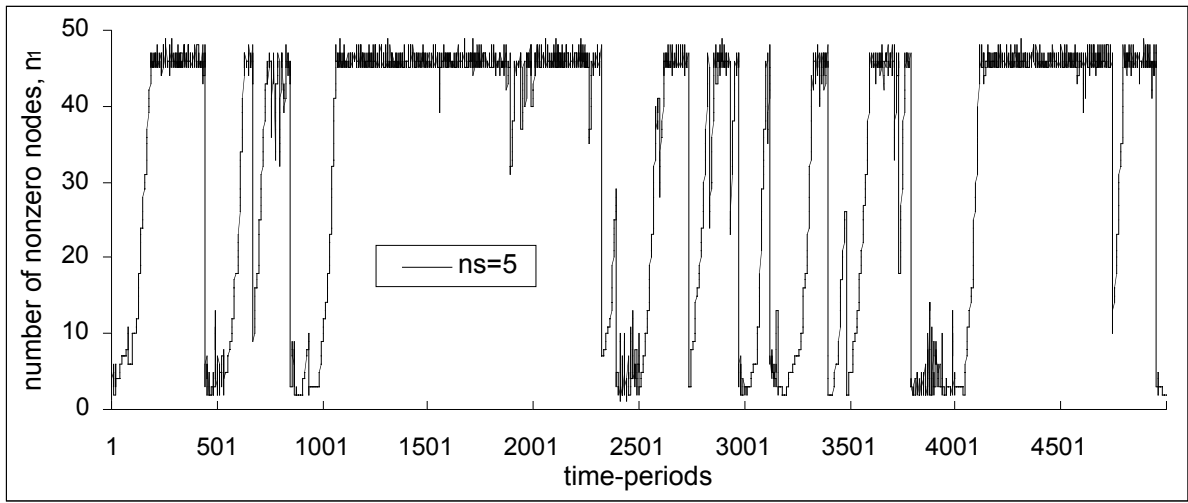

c)

Figure 3.8. Effect of the number of types mutated in one time period (ns) on system behaviour. Connection probability, p equal to 0.005 in the three simulations. 


\subsection{Discussion}

In the simple model presented here, I have attempted to sketch some temporal dynamics of knowledge creation, through the flow of diverse ideas among communities of technological practitioners, over the life cycle of a technology system. Driven by a uniform highly simplified evolutionary dynamic, the system exhibits three distinct phases as it passes from a fluid experimentation phase to a 'mature' fullyconstructed phase. The central event in this life cycle is the emergence of a selfsustaining structure, which acts as the dynamic core of the technology system. The model shows that the presence of the core is a necessary condition for assimilating exogenously introduced novel ideas, and producing a system-wide accumulation of new knowledge. Much of the innovative dynamism underlying this accumulation however is provided by the on-going sustained capacity of the core to generate novel ideas. For example, consider the relationship between the operating system (core) in a computer's software and the secondary software applications (periphery) than can be run on this operating system. The operating system 'assimilates' the new applications often coming from quarters quite unrelated to those responsible for the development of the operating system. The applications no doubt represent 'small' innovations in their own right, however, much of the innovative dynamism in the system as a whole comes from continuous innovation in the operating system itself (as evidenced by the release of newer versions of MS Windows every few years, each version extensively broader in scope than the previous one).

Over time, the system self-organizes to a 'mature' state. This state is characterised by a high degree of interdependence among the parts of the system, and almost all parts are equally innovative. The successful introduction of a new technology (or knowledge type) into this 'mature' system depends upon the degree of relevance of this technology to the existing parts, or how quickly the new technology gets adapted, and successfully assimilated, into the system. New technologies that are highly relevant to the system are the only ones retained. This relevance is established in the model by the structure of connections a new technology establishes with other technologies (e.g. new technologies that are downstream from existing successful technologies get retained more easily). In addition, the ability of the system to assimilate new technologies depends upon the dynamic strength of its core: a strong core, with a large number of internal positive feedbacks between its constituent technologies, acts as a continuous stimulus for innovation in subsidiary or periphery components without losing its own innovative dynamism. Finally, the robustness of a 'mature' system to exogenous shocks is a function of its core-strength. A 'very strong' core allows the system to exclude all possible exogenously introduced radical innovations that can cause a shift in the knowledge accumulation regime. On the other hand, in order to bring about a regime-shift, introducing a new combination of multiple mutuallyreinforcing ideas is likely to be more effective than introducing individual technologies or concepts. 
The above model is just one possible application of the abstract ACS model developed by Jain and Krishna (2002). Nevertheless, the main theoretical underpinnings of the model in core-periphery structures and positive feedbacks in circular processes, and the results on core-strength (or dominant core), ${ }^{28}$ have much wider applicability. In part II of this thesis, these results are used to examine actual networks of a community of farmers in Andhra Pradesh, India. In particular, the theoretical constructs developed in chapters 2 and 3 are used to analyse the structures of knowledge, business and social networks of an Indian village surrounding the development and adoption of a new technology with several underlying knowledge types and constituent practices.

${ }^{28}$ In the empirical chapters, links in the networks are not weighted due to the lack of availability of data. And thus core strength is only a function of the density of internal links. 


\section{Part II}

Social interactions and social capital 


\section{Introduction: networks of a rural community}

In this part II of this dissertation, I study networks of an agrarian community in Andhra Pradesh, India. The aim is to illustrate the empirical relevance of the theoretical concepts developed in the part I of the thesis and to use them to help understand the village. In particular, I use the concepts of circular process and the results on core-periphery structure (auto-catalytic sets) in networks. The empirical evidence presented pertains to three different types of network of one community - a village in southeast India. The first network, a map of problem-solving knowledge flows, is used to examine the nature of knowledge creation and diffusion in the community. Using the second network of the farmers' business transactions with others, the farmers' present structure of dependence (or counter-dependence) on middlemen and farm-inputs suppliers is situated in agrarian history. The final network of social relations focuses on close kinship and friendship ties. This network is used to understand the socio-political activities of the farmers and the power structure in the community. The methodology used for data collection is described in chapter 5 .

In chapters 6, 7, and 8, events and activities surrounding the experience with pest control technologies in the community are analysed using the structures of its knowledge, business, and social networks. In separate chapters, circular processes are identified to determine the core-periphery structure of the three networks of the community. Of all the circular processes in a network, the dominant core is the one with the highest density of internal links (as demonstrated through simulation experiments in the previous chapter). Membership of this dominant core, coupled with other network-analytic measures of power, is used to identify the powerful actors who drive socio-economic and technological activities of the community. A drawback of the empirical analyses of this part II of the dissertation is the lack of an explicit time dimension: I only have network data for one point in time. In contrast to the theoretical model presented in the previous chapter, issues of network evolution cannot be addressed here.

\section{I The community in focus}

The community studied is a village called Punukula in Khammam district of Andhra Pradesh (AP). The village became famous as a pesticide-free zone and gained extensive media coverage in national and regional publications. It was proclaimed as a bio-village and hailed as a model, an ideal village, for other villages to emulate. The following study is largely based on quantitative data gathered through informal interviews and questionnaire-based survey of the inhabitants during several trips to the village between September 2005 and April 2006. Most survey interviews were conducted in the months of February and March 2006. In addition to surveying the village, I interviewed profit-seeking actors from outside the village who supply all farm-inputs and credit to the villagers, and representatives of non-profit organizations who had direct contact with the farmers. 
The studied village has a population of approximately 900 inhabitants. According to the data collected with the survey, 155 households out of a total of 212 are cultivating farmers. 141 of the 155 farming households own some land, and 14 cultivated land leased from others (a few farmers with small landholding also lease land, see Table 4.1 for distribution of landholdings in the village). Most farmers are smallholders who own less than or equal to 2 hectares of land. In addition to the farmers, the village is inhabited by 38 landless farm-labourers whose primary income is derived from agriculture. The remaining households are engaged in non-agricultural professions such as small shop-owners in the village and construction work in a nearby town. A small number of farmers own shops or drive auto-rickshaw taxis in the area. The village is located $6 \mathrm{~km}$ from the nearest town and the closest industrial establishment is a thermal power plant where a few villagers are employed. The main source of income in the village continues to be agriculture which provides employment for approximately $90 \%$ of its population.

Table 4.1 Distribution of land holdings (owned and cultivated) in the village

\begin{tabular}{|l|l|l|}
\hline & Owners & Cultivators \\
\hline Number of farmers with $\leq 1$ hectare & 71 & 48 \\
\hline More than 1 but $\leq 2$ ha & 50 & 51 \\
\hline$>2$ but $\leq 3$ ha & 7 & 26 \\
\hline$>3 \leq 4$ ha & 8 & 20 \\
\hline$>4 \leq 6$ ha & 2 & 7 \\
\hline$>6 \leq 8$ ha & 3 & 3 \\
\hline Total number of farmers & 141 & 155 \\
\hline
\end{tabular}

In the last 7 years (from 2000-2006), with help from two non-governmental organizations (NGOs), many farmers in the village decided to switch from chemical pesticides to alternative pest control methods. These low-cost methods do not require the use of pesticides and may be considered a non-pesticidal version of integrated pest management. The NGOs refer to these methods simply as non-pesticidal management (NPM, see Box 4.1 for a brief history of NPM development and Chapter 5 for a list of different NPM methods and practices). Farmers were recommended to use a few of the methods (or practices) simultaneously to effectively control pests on crops such as cotton and rice. The general model for NPM 'implementation' by the local NGOs involved working closely together with the farmers and enabling farmer-led innovation: It was intended and hoped that the NPM project will lead to the development of an innovative capacity among the farmers in the village to experiment for modifying existing NPM methods to suit their needs, and whenever possible invent new ones. To facilitate this, the NGO's built NPM on existing farmers' knowledge; extensively trained the farmers on all aspects of NPM; identified 'progressive' farmers interested in the NPM alternative and working closely with them; appointed 10-15 influential farmers as the NGO's resource persons in the village; formed a farmers' 
working group (Raithu Mitra Group) which met regularly to facilitate decision-making and knowledge sharing; installed a mill to crush neem seeds locally in the village for preparing neem spray (a critical NPM component) and trained two villagers to operate the mill; and lastly stationed an NGO representative in the village for mobilizing farmers and make NPM-related assistance readily available to the farmers.

The transition to NPM in Punukula has received generous mass media attention since mid-2004. ${ }^{1}$ This transition to an effective low-cost pest control techniques became particularly interesting at a time when thousands of farmers across India were committing suicide due to their inability of repay debts incurred in procuring expensive farm-inputs such as pesticides. Many national and regional newspapers reported that all farmers in the village had stopped using pesticides and the village became a Mecca of sorts for pesticide-free farming. In October 2004, the local government body of the village, the gram panchayat or village council, officially declared that the village is pesticide-free. ${ }^{2}$ Finally in November 2004, the agriculture minister of Andhra Pradesh visited the village along with an entourage of bureaucrats, politicians, academics, NGO workers, and reporters. I provide more details on these events in the following section of this chapter where I recount the story of pest control in the village as reported in the media and the NGO publications. The media, mainly the print media, reported the severity of the pesticide and debt crisis followed by a description of the solution achieved with NPM. Barring one or two exceptions, this pattern of the story was reasonably consistent across all reports (from the media and the NGOs) on Punukula in 2004: an acute crisis and then the solution with NPM leading to a pesticide-free village. $^{3}$

\section{Box 4.1 A short history of NPM}

Non-pesticidal management (NPM) is the result of over a decade of work by a group of NGOs from different parts of Andhra Pradesh. The organization that acted as a hub and provided the necessary financial and knowledge resources in the development process is a Hyderabad-based NGO called Centre for World Solidarity

${ }^{1}$ I have come across at least 15 reports on the non-pesticidal farming-related events in the village in national English language newspapers and magazines. Many more reports appeared in the local language (Telugu) publications in AP. In addition to the media reports, representatives of the involved NGO's authored (and published) early reports of the NPM-related success in the village.

2 This pesticide-free status of the village has at least two different versions. According to the first, that was claimed by the NGO and their supporters, the panchayat resolution banned the use of pesticides in the village. According to the second, that appeared in a local newspaper, the resolution only asked pesticide company representatives not to visit the village anymore.

3 This reported story may have omitted some crucial details or simply reported them incorrectly. However, what I call omitting here may just be a lack of depth in the newspaper reports as a necessary result of the short time-frames in which they must be prepared and printed. Most journalists went to Punukula for a few hours or at most a day to cover the events. But this lack of depth was not limited to newspaper reports: some academics also based their study on Punukula after visiting the area for one or two days (see for example Ghosh 2004). 
(CWS). Other NGO's located in different districts of AP acted as 'implementing organizations'.

CWS is a multi-divisional organization whose activities include strengthening local government institutions in rural areas and campaigning against domestic violence. According to the founder and the present convenor of CWS, Mr. Sastry, a broad theme that runs through most of the organization's activities is helping the rural poor to organize for collective action. The technical expertise in NPM, both in its knowledge for pest control and organizing for collective action aspects, was largely provided by CWS. In 2004, the sustainable agriculture wing of CWS was hived off as a separate NGO called Centre for Sustainable Agriculture (CSA). CSA is a 'knowledge-NGO' as it employs agricultural scientists to perform research and development activities on NPM (see Prasad 2005 on NGO's and knowledge creation). In addition, it carries out policy advocacy work at the national and provincial levels to promote sustainable agriculture. CSA has retained CWS's network of local grassroots based NGO's working on NPM. The local NGO working in the village, Socio-Economic and Cultural Upliftment in Rural Environment (SECURE), is a member of this network.

NPM is based on farmers' knowledge from different parts of south, southeast, and central India and uses materials that are generally locally available in the villages. It is a pest control strategy based on a mix of bio-pesticides such Neem seed kernel extract and other practices such as intercropping. NPM is CSA's central tool in promoting sustainable agriculture. In fact, the 'official' CSA (and CWS) discourse on NPM includes the development of a village economy for pest control through NPM. In this economy, farm-labourers and other landless people in a village collect NPM ingredients (such as Neem) and prepare the bio-pesticides to sell to local farmers.

\subsection{The story of pest control in the village}

The story of intensive pest control in Punukula starts about 20 years ago when some immigrant farmers from the Guntur region of Andhra Pradesh began cultivation in the village (Ramanjaneylu et al. 2004; Kuruganti 2004a). The Guntur farmers were the first ones to cultivate cotton in Punukula. ${ }^{4}$ Witnessing the promise of high cashreturns in cotton, as compared to food crops such as pulses and millets, many native farmers in Punukula soon followed the example of the Guntur farmers. These Punukula farmers got good yields and satisfactory returns in the first few years of cotton cultivation (Ramanjaneylu et al. 2004). But gradually, the pests started

\footnotetext{
${ }^{4}$ Cotton is the most pesticide-intensive crop in India: it accounts for more than $50 \%$ of the pesticide consumption while it is grown on only $5 \%$ of the total cropped area (Joshi 2004; Choudhary 2004).
} 
developing resistance to first generation pesticides used in those days, ${ }^{5}$ forcing the farmers to try newer generations of pesticides recommended by the pesticide dealers. By the end of the 1990's however, pest attacks were not controlled by the latest generation of expensive synthetic pyrethroids. Afraid of losing their crops, farmers sprayed greater quantities of pesticides every season to no avail. This pesticide treadmill and the resulting high cost of cultivation led farmers into a "debt trap" (Ramanjaneylu et al. 2004). The debt trap was exacerbated by the dependence of farmers on the local pesticide, fertiliser and seed dealers (the all-in-one dealers) who are their sources of credit as well as buyers of their crop. The two, supply- and demandside, problems of the farmers signal the start of the most recent crisis in agriculture in Punukula predominantly in the cultivation of cotton. ${ }^{6}$

Almost all reports about the village described the expenses on pesticides as the farmers' main cause of distress. The farmers were reported as buying the pesticides on credit from the all-in-one dealers. Apart from paying a high rate of interest $(2-4 \%$ per month), ${ }^{7}$ farmers were forced to sell their crop to these same dealers at prices up to $35 \%$ lower than the market. This dependence on the dealers acted as a recipe for disaster: low crop prices coupled with high cost of cultivation landed farmers into heavy debts. One Punukula farmer interviewed in the media reports claimed the cost of cultivation to be as high as 60,000 rupees per hectare, whereas the price of cotton crop was reported to be around (generally less than) Rs. 20 per kilogram (Joshi 2004). If the farmer harvested a relatively high yield of 2000 kilograms per hectare, his debts increased by Rs. 20,000 per hectare of cultivated area. Many farmers interviewed in the reports were indebted by more than 100,000 rupees (approx. €1900) (see, for example, the interviews in Joshi 2004).

Another facet of the problem reported was the frequent poisoning in the village due to pesticide use. The debt situation of a farmer was worsened if a farmer or a family member had to be hospitalised due to pesticide poisoning (hospital bills

5 Pests developing resistance to pesticides is a commonly accepted phenomenon in agricultural sciences. The phenomenon is common for pests attacking cotton (Cowan and Gunby 1996). A pesticide kills susceptible members of the pest population while leaving the resistant members alive. The resistant members then reproduce, leading to the development of genetic resistance among the pests. This continual dependence of the farmers on newer generation of pesticides is often referred to as the 'pesticide treadmill'.

6 This agrarian crisis is not restricted to Punukula alone. Many parts of rural India are suffering from a crisis, which is reflected in the high rates of suicide reported among farmers in the last decade. The indebtedness of farmers, due to high costs of cultivation and low crop prices imposed by open markets, is commonly considered the main reason behind the suicides (see for example, Ghosh 2005; TISS 2005; Mishra 2006). The problem is worsened when small farmers are refused loans by (public) banks and have to borrow money at high interest rates from local moneylenders. The worst affected farmers cultivate cotton for which pesticides can constitute $40-60 \%$ of the total cost of cultivation (Ramanjaneylu et al. 2005).

7 This interest rate figure was reported by Ghosh (2004). The Centre for Sustainable Agriculture reported this interest rate as 3-5\% per month (Ramanjaneylu et al. 2005). 
routinely ran into thousands of rupees). Such cases of severe poisoning were experienced at least once a season in Punukula and "people died from excessive exposure.” (Ghosh 2004). Some reports named a villager who was killed due to direct exposure to pesticides and another who committed suicide because of heavy indebtedness (Rao 2004; Ramanjaneylu et al. 2005). The more-routine health hazards of pesticide use were also highlighted in the media reports: both men and women in Punukula suffered from health problems such as skin irritation, nausea, and blurred vision (Ghosh 2004; Kuruganti 2004b). According to a paper on Punukula written by employees of the two NGO's who promoted NPM in the village, Centre of Sustainable Agriculture (CSA) and SECURE (see Box 4.1), one of the two local medical practitioners in the village states that 50-60 cases of pesticide poisoning occurred every year before 2000 (Ramanjaneylu et al. 2005).

In the foregoing, I have detailed the media's description of the severe crisis in Punukula. According to most reports, this crisis began to subside around 2001 when SECURE started implementing NPM in Punukula. During the initial stages of the NPM 'transformation' in Punukula, very few farmers evinced any interest in trying out the new methods. The media reported anywhere between 1 and 20 as the number of farmers who tried out NPM in the first year. Persuading these early adopters to use NPM involved a great deal of effort on part of SECURE, which included speciallyorganized demonstration trips for Punukula farmers to another village where NPM was being used. In due time, the demonstration effect of the good results achieved with NPM by early adopters led other farmers in the village to try NPM. By 2003, all farmers in Punukula were reported as using NPM successfully. Farmers were even reported to be experimenting with NPM and adding new practices to the NPM corpus (which was originally based on farmers' knowledge).

The cost of cultivation had reduced significantly: the cost of pest-control with NPM was Rs. 800-1000 per hectare of cotton cultivation as compared to Rs. 15000 with chemical pesticides (Ghosh 2004). ${ }^{8}$ This reduced cost of cultivation made agriculture profitable again. By September-October 2004, when most media reports were published and Punukula was in its second pesticide-free season of cotton cultivation, farmers were reported to have repaid a large portion of their debts accumulated due to the earlier pesticide-intensive farming practice (see Choudhary 2004 for one such example; also see Joshi 2004; and for a more general village-wide version see Venkateshwarlu 2004, Kuruganti 2004a; and CSA 2006). According to some reports, farmers were not using pesticides on any crop in Punukula and the village had completely rid itself of pesticides. In fact, the village council (gram

8 Others claimed that pesticide expenditure was higher, between Rs. 20000-30000 per hectare of cotton cultivation (e.g. Choudhary 2004). 
panchayat) had adopted a resolution banning the use of pesticides (see for example, Choudhary 2004). ${ }^{9}$

Punukula's success story and the publicity it received made the village famous and a popular destination for farmers and agricultural NGO representatives from different parts of AP. Some visitors came from other parts of India as well. It appeared that everyone was interested in finding out how the village rid itself of pesticides and taking crucial lessons home. The state secretary in the ministry of rural development and the chairperson of the farmer's welfare commission of AP also visited Punukula separately. In November 2004, the agriculture minister of AP made a trip to the village. He was joined by two members of the AP legislative assembly, senior officers from the agriculture department, the district commissioner of Khammam, some academics, and representatives of CSA. The public interest in Punukula continued after visits by these important people. ${ }^{10}$ In fact, my first visit to the village in September 2005 coincided with a visit by a busload of farmers from the Western province of Gujarat.

The foregoing synopsis of the media and NGO reports shows that the media painted a utopian picture of Punukula in the post-NPM phase. Most reports listed an impressive set of benefits arising out of the use of NPM in the village. There were three aspects of Punukula's success with NPM that were brought to the fore in these reports,

NPM was widely used in the village, particularly on cotton but also on other crops such as rice and pigeon pea. NPM use in the village was stable (everyone in the village continued to use pesticides in 2004). NPM was based on farmer knowledge and experimentation. Punukula farmers shared their knowledge widely with other farmers including those visiting farmers from different parts of AP.

NPM permitted farmers to repay their debts accumulated due to pesticideintensive farming in the pre-NPM phase. The farmers were no longer dependent on the local all-in-one dealer for maintenance of their livelihoods. Farmers were not incurring any new debts since they started using NPM. The 800 or 1000 rupees spent on NPM by the farmers were used to acquire locally grown plants or on local labour, thus promoting the development of a village economy for pest control materials.

Farmers reported a dramatic reduction in their health problems; and an improvement in their credit-worthiness with local banks and micro-credit agencies. NPM offered the farmers an opportunity to work together and collective action was

9 As noted in footnote 2 above, there were at least two versions of what this resolution contained. Some other reports claimed that the Punukula panchayat's resolution asked pesticide companies not to send their representatives, as the village was pesticide-free.

${ }^{10}$ Some holes in the utopia-story constructed by the media began to surface in the reports on the minister's visit to the village. A few prominent farmers claimed that, with the exception of 10 farmers, no one was using NPM in the village. Further according to this group of farmers, they stopped using pesticides on their own initiative and SECURE played only a marginal role in the nopesticides success of Punukula. Farmers also reported that they were still indebted and were getting a low price for their cotton crop. They also raised water shortage and labour availability problems in the village. 
made possible in the village. In general, the media projected a quasi-utopian image of the village in the post-NPM phase.

In this part II of the dissertation, I analyse these aspects using data on the three networks on knowledge flows, business relations, and social (kinship, friendship) ties. Chapter 6 traces the rise (and subsequent fall) of NPM in the village by analysing the structure of its knowledge network. In chapter 7, I analyse the 'escape from the clutches of all-in-one dealers' aspect of NPM through the lens of Punukula's business network. And chapter 8 looks at collective action in the village as a feature of its social network structure. In particular, I argue that the content of media reports was partly driven by projection of the face of success and unity collectively by the villagers to the visiting journalists (until the minister's visit). ${ }^{11}$ Similarly, cooperation with the NGO's for NPM would not have been possible without some degree of collective action in this multi-caste village. The methodology for collection of data used in this part is discussed in chapter 5. The final empirical chapter 9 explores the interpenetration of the three networks in each other and its implication for interactive learning in a possible local innovation system.

11 The media reports were also driven by the representation of the events in Punukula by the NGOs involved in the NPM project. My point is that the NGOs could not have managed to project the face of success without extensive corroboration by the villagers themselves. 


\section{Network data and approach used for data collection}

The fieldwork for data collection was done between September 2005 and April 2006 in and around Punukula. A main aim of the fieldwork was to gather information for understanding the social organizational bases of the successes (and failures) of the village as outlined in the previous chapter. This task required a mapping of the social, business and knowledge networks of the village. In order to collect useful data to map the networks, it was necessary to ascertain the different activities of farmers in the three institutional domains of social, business, and knowledge transactions (Srinivas and Beteille 1964). For example, what, according to farmers, constitutes strong social ties; what are the various farm-inputs that they procure; what are knowledge interactions and how can they be separated from other interactions? In addition, terms and categories had to be discovered to ensure that questions asked by an interviewer were effectively communicated to the farmers. In other words, meaning of the terms used must be shared by the interviewer and the interviewee (Converse and Presser 1986). The above mentioned issues were gradually resolved during several trips to the village over the course of 4 months before designing the survey questionnaires which were then used to interview all households in the village. ${ }^{1}$

The questionnaire was then pre-tested to control the length of an interview and to evaluate the quality of information gathered. On average, each interview lasted about 20-35 minutes. Three different questionnaires were eventually designed: one each for farmers, landless farm-labourers, and shopkeepers in the village. ${ }^{2}$ A small number of villagers did not fall in either of the three questionnaire categories, such as employees of a nearby thermal power station. These villagers were interviewed using the farmlabourer questionnaire if they did not own or lease any land, and the farmer

1 Preliminary information was gathered during these trips through informal discussions and interviews with farmers and local NGO representatives. This information was used to finalise the interview questionnaires. To the best of my knowledge, barring a couple of households, representatives from all households in the village were included in this study. Households rather than individuals are the focus here because husband and wife generally cultivate together unlike farms in much of Africa which are individually controlled (cf. Goldstein and Udry 2004). Male head of a household was the first choice as an interviewee because most business transactions of a household with agencies from outside the village (such as pesticide dealers and NGO's) were handled by him. The social network data presented here is also biased toward the husband or the father. In this sense, this study may be criticized for gender bias in all three institutional areas covered.

2 I prepared the questionnaires in English, which were then translated into Telugu, the language most widely spoken in Andhra Pradesh, by two individuals: The first has ten years experience in doing fieldwork and conducting surveys in rural areas of Andhra Pradesh. The second is an experienced Hyderabad-based journalist who hails from a farming family from a village in the same district as the one in which the village under study is located, and is therefore familiar with many local nuances. The Telugu questionnaires were not back-translated into English for checking accuracy but this is not a problem due to the local knowledge and extensive experience of both translators. 
questionnaire if they did. Some villagers such as the local medical practitioners and auto-rickshaw (taxi) drivers were interviewed using the shopkeeper questionnaire (see Appendix A for the questionnaire). Finally, all villagers were interviewed over the course of two months - February and March 2006. In addition to the network data of farmers, the same questionnaire was used to gather information on their land holdings and the nature of NPM adoption.

The use of survey instruments for collecting network data has a long history and a number of problems with the self-reports by the respondents have been identified (see Marsden 1990 for a review). These problems generally pertain to social support or contact networks, although some of the concerns may be relevant for business and knowledge networks. In general, network data obtained through self-report by respondents in surveys may be less accurate than data obtained through direct observation of the type practised by anthropologists (Marsden 1990). In spite of this concern, surveys continue to be a common mode of network data collection (see Giuliani 2005; ${ }^{3}$ Hlebec and Ferligoj 2002 for recent examples). I attempted to mitigate the informant inaccuracy effect by gathering adequate information, in the various exploratory trips to the village, for framing questions that could be effectively communicated to most farmers.

The nature of the data mandated that most questions in the interviews were openended. However, wherever possible, a range of categories of answers was given to the interviewees (a similar approach with categories of people was employed by Lin and Dumin 1986). These categories acted as name generators, aiding the farmers to recall names of their contacts and allowed the interviewer to get more than one answer (names of contacts) to the same question. For example, farmers were asked to provide names in the following categories of people that they approached for getting a loan: big farmers; moneylenders; pesticide/fertiliser dealers; bank officers; or anyone else. Of the three networks, data for the social network was the most difficult to gather and was eventually sourced from an inhabitant of the village. This person is a local NGO representative who had been living in the village for three years when this fieldwork was conducted. ${ }^{4}$ She had worked with the farmers of Punukula every day during the last three years and was knowledgeable about their social networks.

For specifying the boundary of the network(s) to study in the present study, the village under focus provided the nodal reference. My aim was to map the networks of the village, rather than networks in the village, with specific emphasis on farmers and

\footnotetext{
${ }^{3}$ I thank Elisa Giuliani for sharing her survey questionnaire with me. Although her work on networks in wine clusters is quite different and I framed my own questions relevant to NPM and farming in Punukula, Giuliani's questionnaire acted as an important first exposure to (knowledge and business) network survey questions.

${ }^{4}$ In his review of empirical studies on social networks, Marsden (1990) points out that researchers have routinely used a variety of sources in collecting network data including the approach used here. Burt et al. (1980) used a similar approach of getting social network data from one of the informants (Marsden 1990). For more information on the collection of social network data, see section 4.2.3.
} 
their contacts. As I show in subsequent chapters, most business and knowledge interactions of the villagers happen with people residing outside the village's boundaries. This fact necessitated the specification of a boundary of Punukula's networks quite distinct from that of Punukula itself. To allow a comparison of the networks across the three institutional domains, I decided to construct the networks so that a consistency in size and membership across the three networks is maintained. The size and membership of the networks was limited to the first-order zone of all farmers in the village in the business and knowledge domains. In other words, any individual or organization having a direct business or knowledge link to/from a Punukula farmer is a member of all three networks. The social network was constructed by adding the social ties among farmers and their business and knowledge contacts to the social network of Punukula residents proper.

This chapter is structured as follows. I discuss the approach for the knowledge networks, followed by the business and social networks. For the first two types of networks, the network data gives information on unidirectional links or flows whereas the social network data represents mutual support i.e., undirected or symmetric links. The focus of this study is on farming-households, as they constitute approximately $75 \%$ of Punukula's household population. I discuss the part of the questionnaire used to estimate the farmers' innovation capacity (related to the extent of NPM adoption and experimentation) in section 5.4 .

\section{I Approach for collecting data on knowledge-flows in the village}

NPM is a knowledge-intensive system of pest control developed by the Centre for Sustainable Agriculture (CSA, Hyderabad) and its partner NGO's. According to the CSA, NPM methods and practices were largely derived from traditional knowledge of farmers from central and south India. ${ }^{5}$ The CSA considers the future development of NPM as heavily contingent on knowledge creation by the farmers, which in turn depends on experimentation and problem solving by farmers themselves on their fields. The results of the farmers' experiments must then find their way to the representatives of the NGO's for wider development of NPM. This makes reciprocal knowledge flows between NGO representatives and farmers, and among farmers, central to understanding the NPM experience in Punukula. Therefore, it was imperative that farmer-to-farmer and farmer-to-expert (NGO representatives, pesticide dealers, agricultural extension officers) knowledge flows are mapped alongside the knowledge flows to the farmers.

Disentangling knowledge interactions from other everyday interactions of farmers was a difficult task. This disentangling was achieved by focussing on flows of problem-

5 Here I am not implying that traditional (or indigenous) knowledge is different from western scientific knowledge. The distinction between the two is hard to sustain epistemologically or substantially (see Agrawal 1995; 2002). 
solving knowledge. That is, knowledge sought by farmers (and others) when confronted with an acute problem - in this case, a severe pest attack (cf. Udry and Conley 2004, p. 4-5). On cotton, such pest attacks occurred approximately once in 2-3 seasons. In order to effectively map knowledge flows to and from farmers to a heterogeneous group of other actors, farmers were asked to provide names for different categories of people including other farmers, NGO representatives, and pesticide dealers. Note that by including pesticide dealers, I was able to map pesticiderelated knowledge flows in addition to those pertaining to NPM. The focus on the knowledge network data therefore was on knowledge flows for pest control in general. The following questions were asked to capture the knowledge flows to and from an interviewee (a farmer-cultivator),

In the last 3-4 years, when did you face a severe pest attack that was very difficult to control? On which crop?

For controlling this pest attack, who did you ask for advice? Provide names of the individuals (include farmers from this or another villages, NGO coordinators, pesticide dealers, and others such as government officials). In each case, please grade the usefulness of the received advice in solving the problem, ranging from little or no use, quite useful, and very useful.

a. Farmers and farm labourers from Punukula or other villages

\begin{tabular}{|l|l|}
\hline Name & Village \\
\hline & \\
& \\
\hline
\end{tabular}

b. NGO coordinator

\begin{tabular}{|l|l|}
\hline Name & Organization \\
\hline & \\
& \\
\hline
\end{tabular}

c. Pesticide dealers

\begin{tabular}{|l|l|}
\hline Name & Shop location \\
\hline & \\
& \\
\hline
\end{tabular}

d. Anyone else (including government agriculture department extension officers)

\begin{tabular}{|l|l|}
\hline Name & Organization \\
\hline & \\
& \\
\hline
\end{tabular}

Who asked you for advice to tackle this pest attack? Please select from the list below and in each category, provide names of the individuals, their organizations or villages. 
a. Farmers and farm labourers

1. Name and village:

2. Name and village:

3. Name and village:

4. Name and village:

b. NGO coordinator

1. Name and organization:

2. Name and organization:

c. Pesticide dealers

1. Name and shop location:

2. Name and shop location:

d. Anyone else (including government agriculture department extension officers)

1. Name and organization:

2. Name and organization:

In response to each network question, farmers were urged by the interviewer to provide as many names as possible. In order to understand the evolution of this knowledge network, the last two questions were also asked for a pest attack that occurred in 1998-99 when NPM still had not been introduced in the village. A large percentage of the farmers were unable to recall enough details to answer the pre-1999 questions satisfactorily. ${ }^{6}$ This latter data is therefore not presented in this chapter of the thesis. The present analysis is restricted to the 'current' knowledge network.

The data collection discussed above was largely restricted to interviews with Punukula farmers. During the initial exploratory trips, it had become clear that farmlabourers played little or no role in pest-control activities. However, questions about NPM were asked to the farm-labourers: if they took part in NPM activities or took advice from one of the farmers, their responses were included in the knowledge network (see the farm-labourer questionnaire printed in Appendix A). ${ }^{7}$ Finally, the

\footnotetext{
${ }^{6}$ Social network scholars recognize the problems of low response rate and inaccuracy associated with questions within specific time frames (Marsden 1990). Partly in recognition of this problem, I kept the time frame of the set of questions on the 'current' knowledge network as the last 3-4 years. Furthermore, I decided to proceed with the questions on the 1999 pest attack for two reasons: first, initial exploratory interviews with farmers and local NGO representatives revealed that many farmers remembered the uncontrollable pest attacks for a long time. Second, attempting to collect this data was important because it would have enabled an analysis of network evolution, which is largely neglected in empirical work on networks (largely because of difficulties in collecting the data on past networks). The quality and response rate for the 1999 data turned out to be very low. For this reason, the present analysis is also restricted to a 'single' point in time, relating to the most recent pest crisis.

7 I doubt that the fame of Punukula's NPM story in regional and national media (discussed in Chapter 4) introduced a bias into the normal knowledge transmission in the village, leading to an exclusion of farm-labourers. NPM was introduced to the village by local NGO representatives, who were generally quite easily accessible by farmers (at one point, two NGO representatives lived in Punukula). Farmers facing problems with NPM therefore directly approached these 'experts'. In addition, most farmers in Punukula knew which farmers were closely associated with the NGO and/or used NPM for a long time, possibly becoming local NPM experts themselves. So these expert
} 
local NGO representatives who were consulted by the farmers for advice were interviewed (the questionnaire is available in Appendix A). ${ }^{8}$ They were asked to name farmers of the village that they took advice from (if any) and farmers whom they often advised. The NGO representatives named a few farmers that they interacted with most often, as part of the NPM project in the village. However, the NGO representatives (and the pesticide dealers) claimed that almost all farmers took advice from them regarding pest-control problems. Therefore, most direct data used to construct the knowledge network of the village was sourced from the farmers, with the NGO representatives and the pesticide dealers only providing general confirmation of the self-reports by the farmers.

\subsection{Business network data}

Of the three networks, the business network was probably the simplest to map. The focus was on the farmers' sources for credit and different agricultural inputs, and on buyers for their crop. In addition to credit, I identified five important inputs for agriculture: seeds, fertilizers, pesticides, materials for preparing NPM methods, other agricultural implements and tools. ${ }^{9}$ Farmers were also asked about their forward linkage, to whom and where do they sell their crops? In each case, farmers were urged to provide names of the persons they usually dealt with. For the crop-sale and credit questions, a list of categories of people was provided because farmers approached multiple heterogeneous actors for these transactions: for example, farmers sold different crops to different buyers and received credit from banks and local all-in-one dealers. For sources of farm inputs, no categories of people were provided because farmers generally bought these from the all-in-one dealers (shops) in a nearby town.

The business network questions in the farmer-questionnaire are listed below.

When you need a loan, who do you approach? Please select in the list below and name the individual and his organization/village.
a. A big farmer (from this or another village)
b. A moneylender (sabukar from this or another village)
c. A pesticide/fertiliser dealer
d. A bank officer
e. Anyone else

farmers, in principle, could always be approached for problem-solving advice. Moreover, many farmlabourers did not use NPM, which was prepared by the farmers' households themselves. Some farmlabourers did not even know of the existence of the local NGO whose representatives mainly worked with the farmers.

8 Four pesticide dealers from whom farmers sourced knowledge and farm-inputs were also interviewed.

${ }^{9}$ Since the majority of farmers are smallholders, tractors and other heavy machinery are rarely used in the village. Information about buying/hiring this equipment was not captured by the survey. 
Who do you usually sell your crop to? Please select in the list below and name the individuals and their location/organization.
a. A moneylender
b. A pesticide/fertiliser dealer
c. At government market yards (provide location)
d. Other local or outside trader
e. Neighbouring farmer(s)

From whom do you source your farm-inputs? Please provide the name of the individual/organization for each input listed below:
a. Seeds:
b. Fertilizers:
c. Neem seeds (main NPM ingredient):
d. Other NPM inputs:
e. Pesticides:
f. Agricultural implements including tools:

Answers to part f. of the last question about tools and implements did not yield names of specific individuals or shops. More than $95 \%$ of the farmers answered 'different shops' in the nearby town as a response. ${ }^{10}$ As a result, it was not possible to construct this network. In all, six different business networks of farmers were constructed using the data collected with the above survey questions. The six networks included four for the different farm-inputs of seeds, pesticides, fertilizers and NPM (the two categories of NPM inputs were collapsed into one), and one each for credit and crop sale. In response to some of the above business network questions farmers did not provide names of individuals but of organisations such as banks and rice mills. Therefore some nodes in the business network are organisations rather than individuals.

For other villagers such as shopkeepers and farm-labourers, similar questions about credit, and inputs/outputs were asked. However, only the information on credit networks by these villagers is included in the analysis. Villagers whose only income was derived from non-farm activities constituted a little over $7 \%$ of the village. Farmlabourers constituted approximately $18 \%$. Using the responses of all villagers, a credit network for the entire village was constructed that included responses of the farmlabourers and the shopkeepers (the non-farm economy of the village). ${ }^{11}$

Finally, the business network of farmers (ego's) was supplemented with information from the local NGO representatives and pesticide dealers (alters). However, in most cases, the dealers did not remember all Punukula farmers' names (as farmers from many different villages bought inputs from the dealers). A large

10 The same question was also asked to the farm labourers and yielded a similar response as the farmers.

11 A network that included the suppliers of materials sold by the shopkeepers was not generated because these materials did not fall into any of the other four categories of farm inputs. 
proportion of the farmers generally went to one main supplier of farm inputs to the village, which was confirmed by this dealer. Therefore, as for the knowledge network, most business network data is sourced from farmers in Punukula. The general validity of this information was however confirmed through interviews with the alters.

\section{$5 \cdot 3$ Collection of social network data}

In order to map the social network in the village, I focussed on kinship and friendship relations of the villagers. Furthermore, I restricted these categories to close kin and friends. There are two reasons for this focus on close ties: first, the network data collected through individual surveys and direct observation on close ties are generally believed to be of better quality than those on weak ties (Marsden 1990). Second, according the literature on social capital in development (see for example, Woolcock 1998; Szreter 2002), social capital is traditionally associated with close social ties characterized by mutual respect, trust, and long-term obligations. ${ }^{12}$

During the preliminary exploratory trips however, most farmers claimed the whole village (or at least all members of their own sub-caste or tribe) as their friends and relatives. In the same initial discussions, farmers generally claimed to talk about everything with others, including weddings, social problems in the village, labour availability, and agricultural problems. However, after repeated interviews over several months, many of them revealed that most of their discussions took place with a few close friends and relatives. In addition, I gradually learnt that closely related families celebrated important festivals together and helped each other in times of need. At the same time, socio-political support was often mobilized through close social ties. Finally, most villagers tended to differentiate between close friends and close relatives in the village. After these discussions, I designed the social-network part of survey questionnaire making sure that I refer to communication patterns and cultural activities that are associated with 'close' kin and friends rather than with casual social contacts. Since my work focused on households, respondents were asked to name individuals or families who are close relatives or friends.

The questions focusing on kin and friends residing in the village are printed below. A similar third question asked Punukula inhabitants to name their close friends and relatives who live outside the village.

Please name families/individuals in this village who are your close relatives (you help each other in times of need and celebrate festivals together), but own a separate piece of land or live separately if landless. Please provide as many names as possible.

a. Name of the relative (family name, first name):

b. Name of the relative:

c. Name of the relative:

12 The interpretation of social capital here is akin to the one due to Bourdieu (1986), as those features of social relations that provide access to economic and cultural capital. For more on the interpretation of social capital in this thesis, see chapters 8 and 9 . 


\section{d. Name of the relative: \\ e. Name of the relative:}

Please name families/individuals in this village who are your close friends (you help each other in times of need). Please provide as many names as possible.
a. Name of the friend (family name, first name):
b. Name of the friend:
c. Name of the friend:
d. Name of the friend:

Please provide names and villages of your close friends/relatives who live outside this village but you see each other frequently.

a. Name of the friend/relative and village:

b. Name of the friend/relative and village:

c. Name of the friend/relative and village:

The quality of data obtained from the self-reports was poor as most villagers were reluctant to give information about their kin and friends to the interviewers (Marsden 1990 points out other accuracy problems with self-reported data). Therefore, most social network data (for about $90 \%$ of the villagers) analysed here was sourced from a local NGO representative residing in the village for over 3 years (cf. Burt et al. 1980 who used a similar method of data collection, sourced from one of the informants). ${ }^{13}$ This data sourcing from the NGO representative was done roughly in the same period as the survey interviews with the farmers. As most of her work in Punukula was done with farmers, the NGO representative possessed excellent knowledge about the social network of farmers (and shopkeepers) in the village, but her knowledge regarding the social relations of about $10 \%$ of the villagers was limited. These data about the village inhabitants missed out by her was taken from the survey data reported by these villagers. ${ }^{14}$ To ensure the quality of this social network data taken from the surveyresponses, only those self-reports of ego's which were reciprocated by alters were

${ }^{13}$ My observations of the social ties of some villagers during the many weeks that I spent in the village are consistent with the data sourced from the NGO representative. In general, the NGO representative's data is of superior quality than the self-reported (social network) data from the survey: this is consistent with Marsden's (1990: 444-45) review of social network data where he pointed out that direct observation methods "have the advantage of increased naturalness and may yield greater descriptive accuracy" than survey interview methods.

14 This combination was done to make the social network data as complete as possible. Such completeness is important while analysing the network structure including positions of individuals in the network. Also as a result of the numerous trips to the village over a period of several months, I became a familiar figure to many villagers. The familiarity, and the trust it bred, helped to improve the quality of some villagers' responses about their social networks during interviews. The interviewer, who conducted $60-70 \%$ of the interviews, also established this familiarity and trust with the villagers. 
included: In his review of network mapping techniques, Marsden (1990) considered this reciprocity important for the accuracy of social-network data.

In addition to the social network data on inhabitants of the village, strong social ties of the villagers with any NGO representatives, pesticide dealers, representatives of financial institutions (credit sources), and farmers from other villages were also mapped. Once again only those self-reports of Punukula residents that were reciprocated by these outsiders were included in the network. However, if a farmer from another village, or some one else from outside the village, was not interviewed personally, the self-reports of Punukula residents were simply included. The social ties to outsiders, although few in number, allow a consistency in network size and membership with the other two networks (business and knowledge) discussed here (see section 4.0 for definition of network boundary). Therefore, the social network data presented here is as close to a complete network as I could get with the available data.

In a final part of this section, below I turn to the measurement of the farmers' innovation capacity carried out using a part of the survey questionnaire.

\subsection{Measuring innovation capacity: knowledge diffusion and experimentation}

A small subset of the questions asked to the farmers in the survey was geared toward examining the farmers' innovation capacity in the new technology, NPM. The innovation capacity is related to the extent of the farmers' adoption of NPM methods and their experimentation with these methods to modify them or devise new ones. A third facet of innovation capacity concerns the sustainability of NPM use by the farmers: in particular, to ascertain if farmers who used NPM once in the last five years were still using NPM in 2005-6. I discuss the farmers' innovation capacity further in section 4.3.1 below.

Only farmers who used NPM at least once were asked the first questions about the nature of their adoption and experimentation. In the remainder of this thesis, I refer to these farmers who tried out NPM at least once as NPM farmers. I asked the following three questions to the NPM farmers to gauge the extent of their adoption of NPM i.e., the list of NPM practices that they adopted, and their experimentation.

What different types of NPM methods and solutions do (or did) you use? Please indicate in the list below,

- neem seed kernel extract

- cow dung and urine solution

- chilli and garlic extract

- tobacco, rice bran, jaggery solution

- detergent and tobacco solution

- Tulsi, marigold flowers, cow dung, urine, garlic, super potash solution

- $\quad$ powdered kodisha plant-branches

- Musti kaya (Fruit of Strichnos Nuxvomica) solution 
- pheromone traps

- trapper plants such as sunflower, maize, marigold etc.

- delayed planting

- crop rotation

- $\quad$ any other solutions (including mixtures requiring the use of pesticides)

Did you modify any existing NPM methods/solutions or discover any new natural method (for example, a new mixture or a new trapper plant) over the course of your experience with NPM?

If yes, what did you discover, when did you first try it and with what results?

Did you tell any other farmers or NGO representatives about your discovery?

If yes, to whom (please provide as many names as possible)?

In the following chapters, I use the three networks to understand different processes surrounding NPM in Punukula. I start with an explanation of the success and failure, the lack of sustainability, of NPM use in the village using its knowledge network structure. The discussion on business network structure follows. I end with an attempt to explain the media publicity and political processes of the village using its social network. 


\section{Innovation capacity of farmers and the knowledge network}

In this chapter, I attempt to understand the nature of NPM use in the village through the lens of its knowledge network. I use the notion of farmers' innovation capacity in NPM to present my arguments. The NPM innovation capacity of a farmer here is determined by three factors. First, the extent of adoption of NPM methods by a farmer; second, by her ability to experiment to discover new practices and solve problems; and third, by the sustainability of the first two factors. ${ }^{1}$ By focussing on the innovation capacity of individual households embedded in a knowledge network, I can study the nature of interdependence and cooperation for knowledge sharing and innovation among farmers in the village. I argue that knowledge sharing among farmers is essential for building sustainable innovation capacity among them. The knowledge network studied here focuses on knowledge for problem-solving in pest management.

The following is largely a descriptive analysis which attempts to identify the relationship between farmers' innovation capacity and their knowledge sharing at a general level. The main aim is to analyse NPM innovation capacity building among farmers (or the lack of it) in terms of theoretical insights from Chapter 3 on circular processes and core-periphery dynamics. ${ }^{2}$

1 The third factor is highly relevant for techniques such as NPM where the cost of switching back to the old technology (pesticides) is low. The first two factors, the diffusion of transferred knowledge and its integration with the recipients' knowledge through experimentation, are commonly used to define innovation capacity in a range of literatures: from studies on multinational corporations in management studies to agricultural innovation systems (for examples of the former, see Cohen and Levinthal 1990 and Almeida et al. 2002; and Hall et al. 2004 on the latter).

2 As noted in Chapter 3, for a sub-graph to qualify as a core, it must be a circular process. The strength of a core is given by the density of internal links within the circular process (clustering). The structure of a core here is akin to Coleman's (1988) concept of closure in social networks (although Coleman does not directly talk about knowledge flows). By attaching central importance to circular flow and clustering, it may seem that I am adhering to the view that closure/clustering is more important than bridging in a knowledge network. However, the core in my framework also acts as a critical bridge between different peripheral components. Thus, the model in Chapter 3 and the empirical application of its results in the following may be viewed as an implicit syncretization of arguments in network studies about the relative importance of bridging vis-à-vis clustering for successful knowledge transfer: for example, Ahuja (2000) argues that bridging has a negative impact on innovation in chemical industry whereas a position with a number of direct and indirect links has a positive impact; Gonzalez-Brambila et al. (2006) find that bridging across structural holes has no impact on knowledge creation in the exact sciences while a high node degree has a positive influence; on the other hand, building on Burt's (1992) arguments on structural holes, McEvily and Zaheer (1999) show that bridging has a favourable impact on competitiveness in industry and managerial performance in organizations. Finally, Rowley et al. (2000) and Cowan and Jonard (2006) have reconciled the two views by arguing that bridging is more important during the initial exploratory stages of a technology's life cycle rather than the later exploitation stages. Overall, bridging is 
The chapter is structured as follows. In $\$ 6.1$, I present an overview of the thinking on agricultural innovation and the role of farmers in that process. Section 6.2 assesses Punukula farmers' innovation capacity in NPM. In \$6.3, I present the knowledge network of the village and connect it to the innovation capacity of farmers. A discussion concludes the chapter.

\section{I Agricultural innovation: a brief overview}

Promotion of agricultural innovation for modernization, higher yields, poverty eradication, and economic growth has occupied policy makers and academics in developing countries since the 1950s. Since the 1960s, numerous agricultural technologies have been developed by the National Agricultural Research Systems (NARS) of developing countries, often in collaboration with international research centres of the Consultative Group on International Agricultural Research CGIAR (on NARS, see Byerlee 1998; on the structure and performance of the Indian NARS, Mruthyunjaya and Ranjitha, 1998; on CGIAR innovations, see Sechrest et al. 1998). Technologies developed by scientists in research laboratories and testing plots of NARS (and CGIAR) are transferred to farmers working in their fields by the agricultural extension service. In the last two decades, this 'top-down' technology transfer approach to agricultural innovation has been severely criticized by development researchers and practitioners alike, who call for a participatory approach to technology development (Farrington and Martin 1988; Chambers et al. 1989; Biggs 1990; Thompson and Scoones 1994; Douthwaite et al. 2001). ${ }^{3}$

Three main streams can be identified in these participatory technology development studies. The first set of studies simply calls on the agricultural research systems to involve farmers as stakeholders in the innovation process, if only for adapting a laboratory-developed innovation to local farming conditions (see for example, Pal and Byerlee 2003). The second set puts 'farmers first' by focusing on the grassroots level where farmers' experiential and traditional knowledge is the main route to producing successful agricultural innovations (see for example, Chambers et al. 1989; Thrupp 1989; Sherwood and Larrea 2001). In these farmer-centred participatory approaches, some farmers act as experts who may train other farmers on a new technology (in farmer field schools, FFS, for example), and thus create a community-

considered more important in areas where enhancing cognitive diversity is critical (as in early stages of a product's development), whereas clustering may be more important for building cohesiveness and joint problem-solving (Özman 2005). In the following, I study innovation capacity building among farmers. For continuous learning that undergirds capacity building in a multi-component technology such as NPM, the combined importance of both bridging (for accessing diverse types of new knowledge) and clustering (for collective problem-solving) structures in knowledge networks is evident.

3 The new participatory approach with its emphasis on farmer-scientist collaboration has its critics as well (see for example, Bentley 1994; also see Thomson and Scoones' 1994 careful espousal of the participatory approach). 
based innovation capacity on the basis of knowledge sharing among farmers (see Fakih et al. 2003 on the FAO-funded Community IPM initiative in Indonesia; also see Wu and Pretty 2004 on farmer innovation circles in China). The third set of studies places equal emphasis on farmers' and scientists' knowledge, highlighting that integrating diverse types of knowledge at all stages of the innovation process is the key to success (see for example, Merrill-Sands et al. 1991; Thompson and Scoones 1994; WatersBayer and Bayer 2005). ${ }^{4}$ The latest avatar of this second line of thinking is the agricultural innovation systems framework, which pays attention to a wide range of individuals, organizations and institutions that play a role in developing agricultural innovations (Hall et al. 2003; Spielman 2005; IBRD/WB 2006). Interactions between different individuals and organizations are deemed critical for continuous learning and innovation success in an innovation system. The components of such a system include the NARS, private firms, civil society organizations, farmers, and the different informal institutions such as habits and routines of the actors. Here farmers, scientists, and other actors either establish channels, or use existing ones, to access knowledge from different actors in the system. The accessed knowledge may then be integrated with one's own knowledge and/or practices..$^{5}$

In an innovation system, innovation capacity (or learning capability) is built at the level of individuals, their organizations, and the system as a whole. ${ }^{6}$ In the following, I focus on innovation capacity of individual farmers who are embedded in the knowledge network of the village, which includes other actors deemed important in a local innovation system such as extension officers from NGOs and the government agricultural department. ${ }^{7}$ I consider the innovation capacity of farmers as the starting point in building capacity at higher levels of organization (and in other domains). This approach to innovation capacity may be justified on three grounds. First, farmers' innovation capacity is critical for defining the capacity at higher levels of organization due to the embeddedness of farmers in networks of the type studied here. Second, as

\footnotetext{
${ }^{4}$ Success here is often defined as meeting the needs of small farmers and poverty reduction potential of a technology.

${ }^{5}$ Knowledge exchange between farmers and 'experts', however, remains restricted due to barriers of 'social' and geographical distance, expertise-based hierarchy, dearth of effective knowledge brokers (scientists, extension agents, or farmers can potentially play this role), lack of embeddedness of knowledge flows in social relations characterized by mutual respect and trust, and the widespread belief that farmers' knowledge is essentially 'local' as opposed to 'global' scientific knowledge. I address some of these issues, using the three networks of the village, in Chapter 9.

6 As highlighted by Hall (2006), building innovation capacity in an innovation system involves several domains including basic and applied research, regulatory institutions, and science/technology policy. He also points out that compartmentalising this innovation capacity and focussing on the individual domains at a time may not be fruitful. However, holistic analyses of the type recommended by him often lack critical details that help us understand the nature of innovation in different domains. $\mathrm{My}$ focus in the following is on the role of farmers in the research and extension domain (knowledge creation and diffusion).

7 There are a total of 10 NGO representatives, 1 government extension officer, 1 agricultural university scientist and 8 pesticide dealers in the village's knowledge network.
} 
the aim of an agricultural innovation system is meeting the innovation needs of farmers, the latter must have the capacity to define and act on their needs for the system to be effective. Third, in participatory projects such as NPM in Punukula, innovation capacity building among farmers is a central aim.

\subsection{Farmers' innovation capacity in NPM}

I define the Punukula farmers' innovation capacity in NPM knowledge and practices using three factors. The first one relates to the 'degree' to which a farmer used the set of NPM practices i.e., the number of NPM methods used. To control pests effectively on crops like cotton and rice, seven complementary NPM practices were considered essential by expert farmers and NGO representatives. ${ }^{8}$ The second factor accounts for experimentation by a farmer to devise new, or modify existing, NPM methods and his attempts to share results of the experiments with others. The third factor relates to the sustainability of NPM adoption by a farmer i.e., the number of seasons during which she used NPM for pest control. ${ }^{9}$ This factor is critical because the costs of switching back to pesticides are low.

The earliest year of NPM adoption by a farmer in Punukula was 2001 when nine farming households used NPM. Gradually, more farmers started trying out NPM. Of the 155 farming households in Punukula, 72 reported using NPM in one of the seasons from 2001 to 2005, or a total of 72 NPM adoptions. ${ }^{10}$ I call these farmers as NPM farmers. On the time of adoption, I have information on only 55 out of the 72 NPM farmers. These data are plotted in Figure 6.1a below. Note that the annual rate of adoption (number of new NPM adoptions each year) makes a big initial jump from 2001 to 2002, but the jumps get smaller in subsequent years with only 4 new farmers trying out NPM in 2005-6. This aspect of the results is clearer in Figure 6.1b.

\footnotetext{
8 These seven practices are: neem seed kernel extract; cow dung and urine solution; chilli and garlic extract; pheromone traps; trapper plants such as sunflower, maize, marigold etc.; delayed planting; and crop rotation. The NPM package brought in by the NGO's was however not limited to these 7 practices only, farmers could use others out of a list 12-14 available methods.

9 An important aspect of innovation capacity of farmers emphasized in the FFS approach is the ability of a farmer to train other farmers. I could not include this aspect because Punukula farmers did not train each other. Also a more general definition of a farmer's innovation capacity, going beyond the present focus on the domain of technological knowledge, may include the farmer's financial capacity (landholding and ability to access credit and labour), access to natural resources such as neem trees (source for a critical NPM ingredient); and aspects of social status.

10 Of these 72 farmers, in seasons subsequent to using NPM, some either switched back to pesticides or did not use any pest control technology. This implies that the maximum number of NPM farmers in one season was much smaller than 72 .
} 


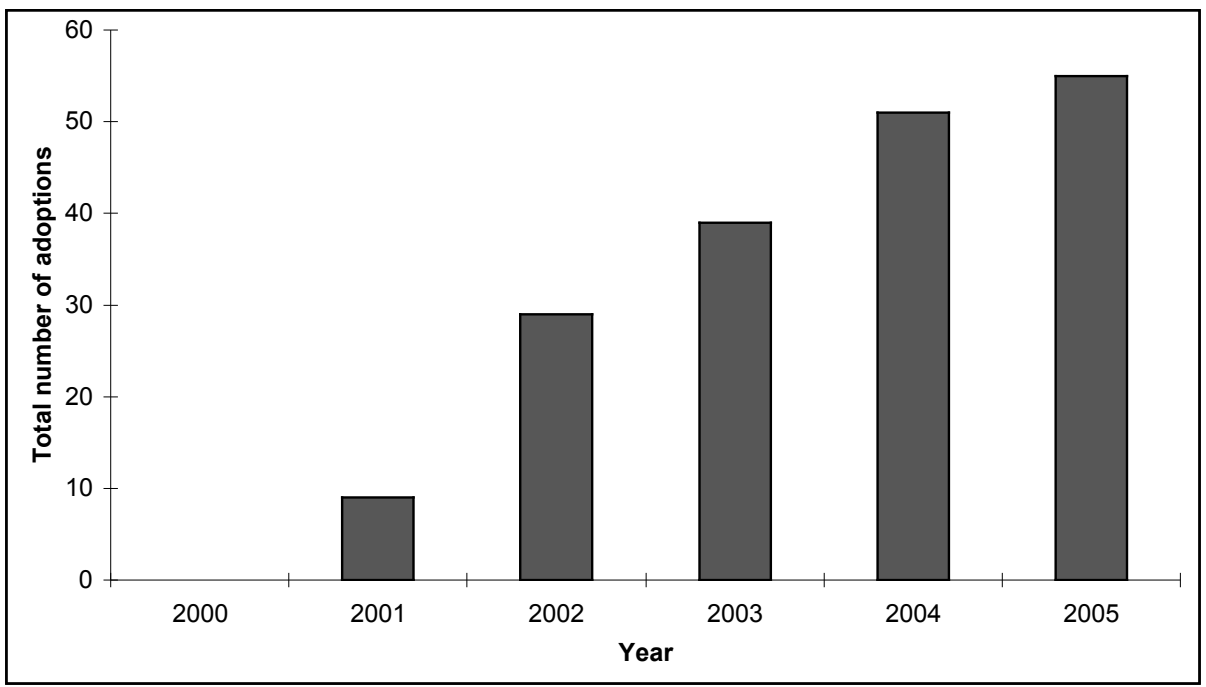

Figure 6.1a Total number of NPM adoptions in Punukula over time.

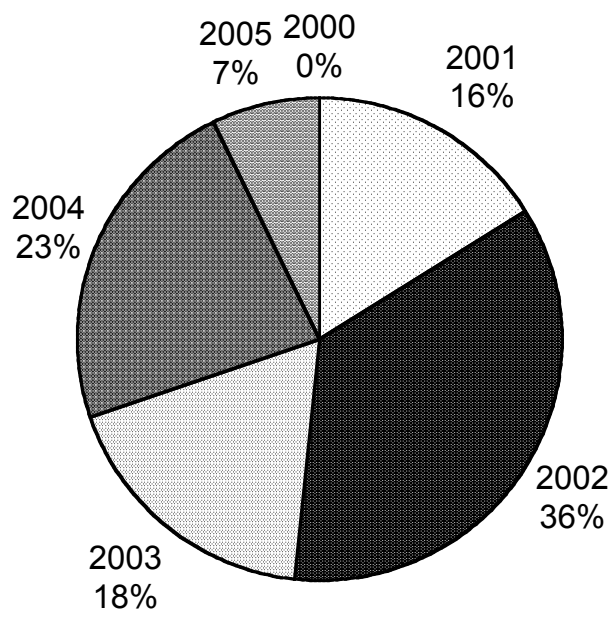

Figure 6.1b Percentage of NPM adoptions each year out of a total of 55 recorded in 2005-6.

71 of the 72 adopting households responded to the question in which they were asked to identify the NPM practices they used. Of the 7 (essential) complementary practices constituting a 'complete' NPM strategy, the 71 NPM farmers used an average 
of 3.17 practices with a standard deviation of 1.87). A majority, 43 of the 71 or $60.6 \%$, of NPM farmers used 3 or fewer of the NPM practices. The maximum number used was 8 , by only two farmers. And only 4 farmers used 7 methods. Therefore, the extent of NPM adoption was limited in two senses. First, less than half the farmers in Punukula used NPM in one of the five seasons from 2001-05. Second, the number of NPM methods used by most farmers was much smaller than that considered necessary for effective pest control. ${ }^{11}$

In terms of ability to experiment, only six farmers in Punukula claimed to have modified existing, or developed new, NPM methods. All six farmers maintained that they received good results in the fields from the use of the results of their experiments. Out of the six farmers, three claimed to have developed the same practice: a mixture of neem extract with detergent. One of the three admitted to having heard about this practice from a farmer in the neighbouring village of Pullaigudem. Furthermore, during my interviews, two NGO representatives working with NPM in the village claimed to have developed the same method and recommended it to the farmers. Thus, it is not clear whether this innovation was farmer-led.

The other three farmers experimented with and tried out three novel ways to control pests not recommended by the NGO's as part of the NPM package. I consider these latter three methods as reliable indicators of experimentation by the farmers. However, only two of the three farmers shared the results of their experiments widely with other farmers in the village. ${ }^{12}$ And only one farmer informed the NGO representatives of the new method he developed (and the good results he achieved). Here two things about the farmers' NPM experimentation efforts are important to notice. First, experimentation, considered essential for farmer-led innovation, was marginal in Punukula. Second and more important, only one successful experimenting farmer shared her knowledge with an NGO representative. Therefore, channels for knowledge transfer from the farmers to the NGO's were not functioning, ${ }^{13}$ thereby limiting the farmers' contribution to the wider development of NPM methods. This lack of knowledge sharing between farmers and NGO representatives also rules out any possibilities for participatory co-invention or integration of farmer knowledge with those of the 'experts'.

In the last season before this fieldwork was conducted, Kharif 2005-6, 32 of the 72 farmers who tried out NPM were still using NPM on at least one of their crops, while only $19(26.4 \%)$ of these farmers used NPM on all their crops. ${ }^{14} 24$ farmers were using

\footnotetext{
${ }^{11}$ Exceptions to the rule of 7 or more practices as 'complete' NPM are however possible here. In cases where a few experimenting farmers, after making minor adaptations to the NPM methods used by them, may have found fewer (than 7) practices as providing effective pest control. But as I show below, there was very little experimentation by farmers, thus possibility of this scenario was marginal. 12 The third farmer reported as informing only one other farmer about his experiments.

13 See footnote 4 above on barriers to knowledge exchange between farmers and 'experts'. Similarly, sharing of new knowledge and interactive learning among farmers in Punukula was also limited.

${ }^{14}$ Data on NPM use on different crops is available only for this latest season.
} 
pesticides again on one or more crops they cultivated and 10 of these used pesticides on everything they cultivated. Twelve farmers used pesticides and NPM both on one crop. Finally, 34 farmers used no pest control on at least one of their crops, but only nine out of the 72 NPM farmers had completely stopped using pesticides. ${ }^{15}$ The above information is displayed in Table 6.1 a below (figures in parentheses are simply percentages of the total 72 farmers).

Table 6.1 Pest control technology used by the 72 NPM farmers during 2005-6

\begin{tabular}{|l|l|l|l|l|}
\hline & $\begin{array}{l}\text { Sprayed } \\
\text { pesticides }\end{array}$ & Used NPM & $\begin{array}{l}\text { Used NPM } \\
\text { and sprayed } \\
\text { pesticides }\end{array}$ & $\begin{array}{l}\text { No pest } \\
\text { control used }\end{array}$ \\
\hline $\begin{array}{l}\text { On at least one } \\
\text { crop }\end{array}$ & $24(33.3)$ & $32(44.4)$ & $12(16.7)$ & $34(47.2)$ \\
\hline On all crops & $10(13.9)$ & $19(26.4)$ & $6(6.9)$ & $9(12.5)$ \\
\hline On cotton* & $13(22.4)$ & $19(32.8)$ & $10(17.2)$ & $16(27.6)$ \\
\hline
\end{tabular}

*Only 61 of the 72 NPM farmers grew cotton, of which 58 reported the pest control technique used during 2005-6.

On individual crops, 23 of the 72 NPM farmers used any pesticides on cotton, which is generally the most pesticide-intensive crop. Cotton and rice were the two main crops that were cultivated, by 61 and 58 of the 72 farmers respectively. A third popular crop was pigeon pea, grown by 31, and chilli was grown by 11 of the 72 NPM farmers. The four main crops and the pest control technique used by the 72 farmers who tried out NPM are listed in Table 6.2a. The numbers in the parentheses are percentages. The same details for all farmers in Punukula are presented in Table 6.2b. The figures show that the third factor of innovation capacity was poor: NPM adoption was not sustainable for the majority of NPM farmers in Punukula. On the two main crops of rice and cotton, only about a third of the original NPM farmers continued to use NPM in 2005-6. On pigeon pea, this fraction was even smaller at about a fifth. Only on chilli, a substantial percentage (60\%) of NPM farmers continued to use NPM. This was largely due to an organic chilli programme run by SECURE in Punukula, in which NPM was the recommended pest control technology. ${ }^{16}$

15 The argument goes that using NPM allows farmers to restore the ecological balance on their fields. This balance between beneficial and harmful insects then allows the farmers to get good yields without using any pesticides. By comparison, a larger proportion of farmers who did not try out NPM, had stopped using pesticides on all crops in 2005-6 (as discussed below).

16 The organic chilli programme was contracted out to SECURE by a Mumbai-based agro-export house called Suminter Organics. A Suminter employee stationed at SECURE was responsible for supply of some organic farm-inputs, farmer-training, ensuring the use of organic methods, and procuring of the crop. The crop harvested in Punukula was to be sent to Suminter in Mumbai, from where, after quality control, it was to be exported. 
In addition, 28\% of NPM farmers had completely stopped using pesticides on cotton. This may have been made possible due to the NPM-driven restoration of the ecological balance between beneficial and harmful insects on their fields. However, note that a much higher $46 \%$ of non-NPM farmers (31 out of 67 ) stopped using pesticides completely without enjoying the expected ecological advantages afforded by NPM use.

Table 6.2a Crops grown by the 72 NPM farmers in Punukula in kharif 2005-6

\begin{tabular}{|l|l|l|l|l|l|}
\hline $\begin{array}{l}\text { Crop } \\
\text { grown }\end{array}$ & $\begin{array}{l}\text { Number of } \\
\text { cultivating } \\
\text { farmers }\end{array}$ & $\begin{array}{l}\text { Sprayed } \\
\text { pesticides }\end{array}$ & Used NPM & $\begin{array}{l}\text { Used NPM } \\
\text { and } \\
\text { pesticides }\end{array}$ & $\begin{array}{l}\text { No pest } \\
\text { control } \\
\text { used }\end{array}$ \\
\hline Cotton & 61 & $13(22.4)$ & $19(32.8)$ & $10(17.2)$ & $16(27.6)^{*}$ \\
\hline Rice & 58 & $21(37.5)$ & $19(33.9)$ & $6(10.7)$ & $10(17.9)^{\varphi}$ \\
\hline Pigeon pea & 28 & $1(3.6)$ & $6(21.4)$ & $0(0)$ & $21(75.0)$ \\
\hline Chilli & 11 & $0(0)$ & $6(60.0)$ & $1(10.0)$ & $3(30.0)^{\zeta}$ \\
\hline
\end{tabular}

* Information on pest control technique only available for 58 of the 61 cotton cultivating-households.

$\varphi$ Information available for 56 of the 58 households.

$\zeta$ Information available for 10 of 11 households.

Table 6.2b Crops grown by farmers in Punukula: data on 152 households

\begin{tabular}{|l|l|l|l|l|l|}
\hline $\begin{array}{l}\text { Crop } \\
\text { grown }\end{array}$ & $\begin{array}{l}\text { Number of } \\
\text { farmers } \\
\text { who } \\
\text { cultivated }\end{array}$ & $\begin{array}{l}\text { Sprayed } \\
\text { pesticides }\end{array}$ & Used NPM & $\begin{array}{l}\text { Used NPM } \\
\text { and } \\
\text { sprayed } \\
\text { pesticides }\end{array}$ & $\begin{array}{l}\text { No pest } \\
\text { control } \\
\text { used }\end{array}$ \\
\hline Cotton & 128 & $49(39.2)$ & $19(15.2)$ & $10(8.0)$ & $47(37.6)^{*}$ \\
\hline Rice & 119 & $64(54.7)$ & $19(16.3)$ & $6(5.1)$ & $28(23.9)^{\varphi}$ \\
\hline Pigeon pea & 63 & $11(17.5)$ & $6(9.5)$ & $0(0)$ & $46(73.0)$ \\
\hline Chilli & 12 & $1(9.1)$ & $6(54.5)$ & $1(9.1)$ & $3(27.3)^{\zeta}$ \\
\hline
\end{tabular}

* Information on pest control technique only available for 125 of the 128 cotton cultivating-households.

$\varphi$ Information available for 117 of the 119 households.

$\zeta$ Information available for 11 of 12 households.

To summarize, the foregoing demonstrates that the farmers' innovation capacity, as indicated by the extent of knowledge diffusion, experimentation with NPM, and sustainability of NPM use, was limited in Punukula. Although $45 \%$ of the farmers tried out NPM in at least one season between 2001 and 2006, a majority of them used 3 or fewer of the 12 practices recommended as part of NPM. The average number of practices used by a Punukula farmer was 3.17, whereas according to the expert NPM farmers and NGO representatives (including the original assemblers/developers of 
NPM at CSA), seven practices are required to achieve effective pest control with NPM on cotton. Thus, the first component of innovation capacity was severely limited. For the second component, only 3 farmers experimented with novel practices of NPM. Of these 3, only 1 farmer shared his new knowledge with a NGO representative. Thus, farmer-input in the development of NPM in Punukula was almost non-existent. On the third component of sustainability of adoption, by 2005-6 only 19 (26.4\%) NPM farmers used it as a pest control strategy on all crops they cultivated (and 9 had stopped using pesticides completely). Thus, the third component of innovation capacity was also restricted to a few farmers (19 out of a total of 152 farmers in Punukula as a whole).

In the remainder of this chapter, I use a map of Punukula's knowledge network to delineate some structural reasons behind the farmers' limited success in developing innovation capacity for pest control using NPM. I use results on core structures and circular processes from the model presented in Chapter 3 to present my arguments. Note that in the following I am not seeking any statistical correlations between the three facets of innovation capacity and the knowledge network structure: My aim is to illustrate an empirical application (and thus provide a first validation) of the simulation results of Chapter 3.

\subsection{The knowledge network}

As discussed in $\$ 6.1$, a central feature of the agricultural innovation studies of the last two decades has been their emphasis on innovation through interaction. These knowledge interactions include farmer-to-farmer knowledge flows, exchange between farmers and other agricultural actors, and interactions between a diverse set of offfarm actors. Therefore, understanding the innovation capacity of a farmer requires paying attention to her connections with actors such as other farmers, suppliers of agricultural inputs, civil society organizations, and state departments/ organizations. These knowledge flows can be captured by mapping knowledge networks constituted by farmers and representatives of other organizations. Such a map of the knowledgeflow network for pest control, focussing on farmers from Punukula is shown in Figure 6.2. The arrows in the Figure represent knowledge flows. Punukula residents are represented by solid nodes whereas the outside actors are shown as unfilled nodes. In addition, all farmers in the network from Punukula and other villages are represented using circles; the NGO representatives are drawn as squares; and the pesticide dealers are the triangles.

This representation of knowledge flows makes it very clear that NGO representatives and pesticide dealers are central actors in this network. Indeed they have the highest out-degree centrality, i.e. they are the most important sources of knowledge in the network. The actors with the ten highest values are listed in Table 6.3. A high out-degree centrality implies that an actor is an important source of knowledge in the network: others take problem-solving advice from this actor. The most popular choice of farmers for sourcing knowledge is the main pesticide supplier 
to Punukula, the all-in-one dealer M Raj (these patterns are clearly discernible in Figure 6.2). Four of the ten actors with highest out-degree centrality values are representatives of the local NGO, SECURE. There are only 4 farmers in the top ten knowledge sources.

Seven Punukula farmers are among the ten people with the highest in-degree centrality, i.e. the people who seek advice from the largest number of other people in the network. Three NGO actors are also members of this set with the highest in degree centrality. These three NGO representatives feature in this list partly due to the knowledge sharing among NGO representatives (ten of their nineteen incoming links are from NGO actors, whereas only nine originate in 7 out of the 155 Punukula farmers).

Table 6.3 Knowledge network members with high degree centrality

\begin{tabular}{|l|l|l|l|}
\hline Name & $\begin{array}{l}\text { Out-degree } \\
\text { centrality }\end{array}$ & Name & $\begin{array}{l}\text { In-degree } \\
\text { centrality }\end{array}$ \\
\hline Raj, M. (all-in-one dealer) & 0.2801 & Payal, D, SECURE & 0.0248 \\
\hline Meka, B, SECURE & 0.1206 & Perumal, E, SECURE & 0.0248 \\
\hline Neha, C, SECURE & 0.0638 & Selvi, FF & 0.0248 \\
\hline Yarla, FQ & 0.0567 & Naidu, CY & 0.0213 \\
\hline Addala, A, SECURE & 0.0497 & Koli, ED & 0.0213 \\
\hline Pillai, GB & 0.0319 & Meka, B, SECURE & 0.0177 \\
\hline Gowda, CL & 0.0248 & Seri, AT & 0.0177 \\
\hline Perumal, E, SECURE & 0.0213 & Datla, BT & 0.0177 \\
\hline Raman, L, Extension officer & 0.0213 & Moti, CQ & 0.0177 \\
\hline Gorinta, EG & 0.0177 & Sapna, EW & 0.0177 \\
\hline
\end{tabular}




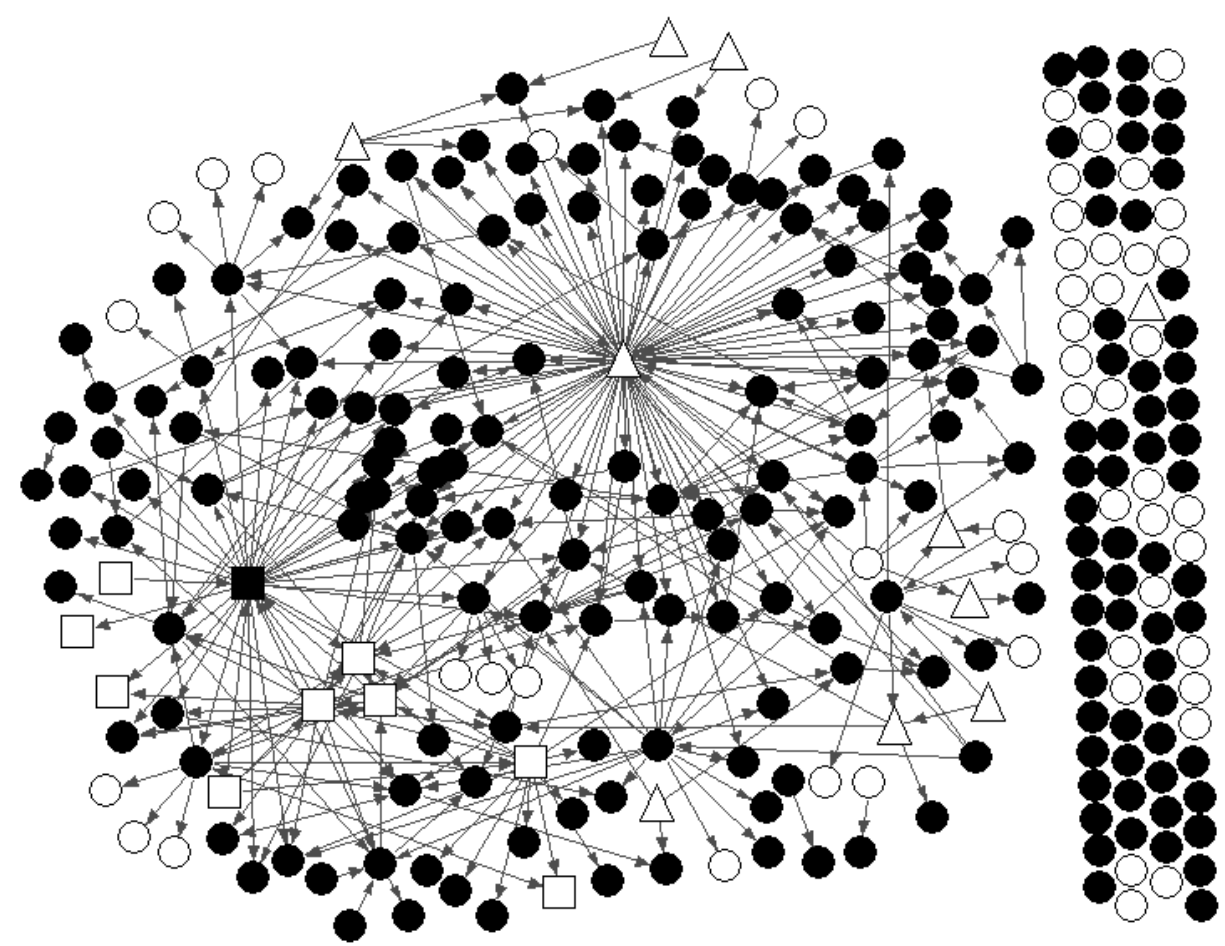

Figure 6.2 Punukula's knowledge network: village residents depicted as black nodes and nonresidents as unfilled nodes. Arrows are knowledge flows. Note the centrality of pesticide dealers and NGO representatives (depicted as triangles and squares respectively) as knowledge providers. Nodes on the right side of the Figure are isolates in the knowledge network.

\section{Core-periphery structure and circular flow}

According to the simulation model of Chapter 3, core-periphery structure of a network depends on the presence of circular flows. For a sub-graph (a sub-network, with 2 or more nodes) to act as a core in the network, it must posses a cyclic structure. A cycle is a graph-theoretic term for circular flow between a set of nodes. A large number of circular processes, or cycles, exist in Punukula's knowledge network. ${ }^{17}$ In Figure 6.3, these cycles are shown in a reduced version of the knowledge network of Punukula. This reduced version only includes those nodes that have at least one incoming and one outgoing link as only those nodes can form a cycle.

17 I counted at least 54 cycles (cores) in the network. All cycles in Punukula's knowledge network were identified using a computer program in $\mathrm{C}++$. The code for this program is available on request. The program is in two parts. The first part reduces Punukula's knowledge network to nodes that have at least one incoming and one outgoing link (as a node can only be part of a cycle if it has these two links). The second part takes the reduced network as input and does a depth first search (DFS) on it to find paths (Eppstein 2007, http://www.ics.uci.edu/ eppstein/161/960215.html). All paths that start and end in the same node are cycles. 
Only one of these cycles was constituted by Punukula farmers alone: a cycle of size two between two farmers (who are brothers). All other cycles included at least one NGO representative or pesticide dealer (as shown in Figure 6.3). Therefore, with the exception of a 2-node cyclic core, the farmers themselves do not form any cores in Punukula's knowledge network. All innovative dynamism, derived from strong cores, in Punukula is provided by cyclic structures formed by,

1. NGO representatives and farmers;

2. only NGO representatives; and

3. farmers and the main pesticide/fertiliser dealer.

This lack of a (strong) core among the farmers demonstrates the absence of sustainable knowledge flows among farmers themselves, which is consistent with a limited innovation capacity among Punukula farmers. ${ }^{18}$ In addition, a majority of the farmers, in the periphery of the knowledge network, are dependent on the dominant cores formed with the help of pesticide dealer and NGO representatives for their innovative dynamism or innovation capacity. Therefore, I do not find evidence for farmer-centred innovation in this village, where knowledge exchange among farmers forms the basis of agricultural innovation. Thus the second of three streams of participatory technology development discussed in section 6.1 is not valid for NPM in Punukula. The structure of the present knowledge network seems more in line with the first and the third approaches discussed there: on farmer adaptation of a technology developed elsewhere and the interactive model in which multiple knowledge actors contribute 'equally'. More details on the structure of the knowledge network and its relation to the farmers' innovation capacity are discussed in the following.

18 In particular, the poor performance in the second component of farmer experimentation and dissemination of results is consistent with the absence of a strong core among farmers in the knowledge network. 


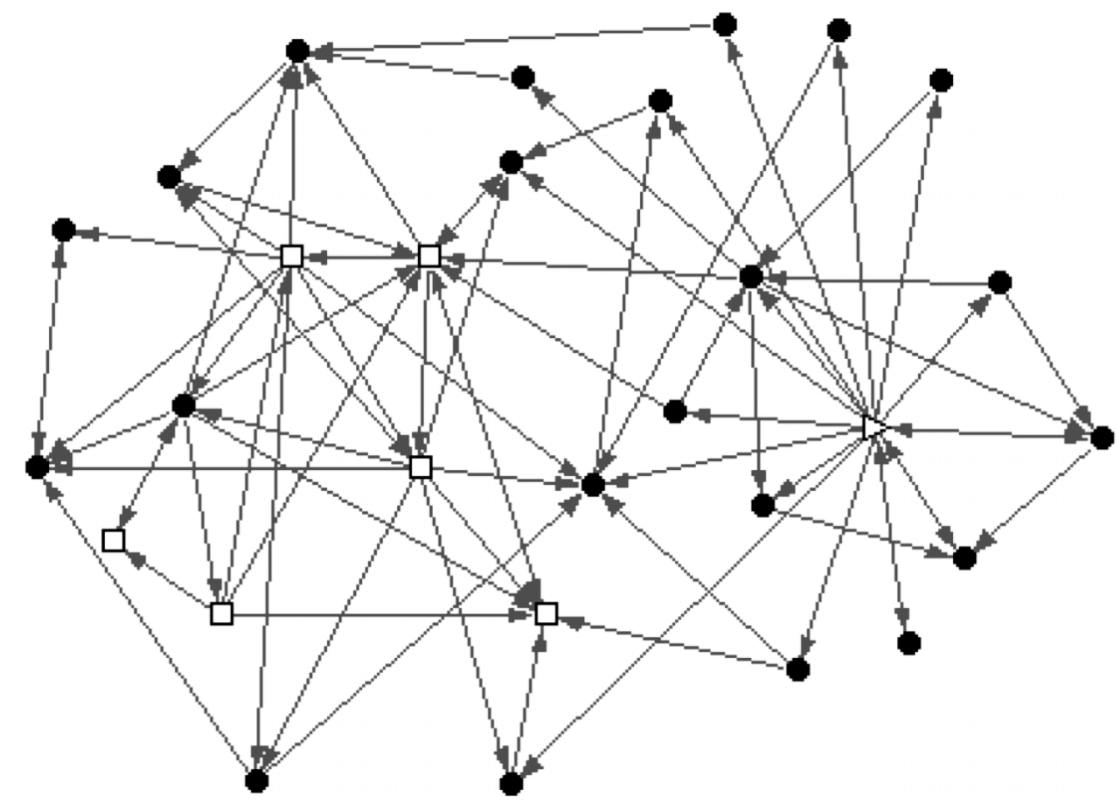

Figure 6.3 All cycles (or cores) in the knowledge network of Punukula (only those nodes which may be part of a cycle are represented here).

As shown in chapter 3 , the dominance of a core in the network is a function of its strength, which is in turn a function of the density of internal links within a circular process. A simple cycle without any internal links (the weakest core) is the minimum condition for a core structure. The strength of a core increases with the density of internal links in the core. See section 3.4 for a more detailed discussion on core strength. If $\mathrm{n}(>2)$ is the size of a core and $\mathrm{n}_{\mathrm{L}}$ is the total number of links among members of the core, the density of internal links of a circular process in directed networks is given by,

$$
d_{i L}=\frac{n_{L}-n}{n(n-1)-n}
$$

For a simple cyclic core, density of internal links $\left(\mathrm{d}_{\mathrm{iL}}\right)$ is zero and for a complete core, it is equal to 1 . The two strongest cores in Punukula's knowledge network are shown in Figure 6.4. ${ }^{19}$ The first one is constituted by NGO representatives and farmers, and the second includes some other farmers and the main pesticide supplier to Punukula. The solid (filled) nodes in the two cores represent farmers, and off-farm

19 These two strong cores were identified out of a total of 54 cycles (or cores) present in the knowledge network. All other cores are either simple cyclic formations or cycles with internal links that have a smaller density $\left(\mathrm{d}_{\mathrm{iL}}\right)$ than the two dominant ones, which indeed posses the two highest values of strength as discussed below. 
actors are shown as unfilled nodes. The density of internal links for the first core, shown in Figure 6.4a, is 0.33. For the second core shown in Figure 6.4b, the $\mathrm{d}_{\mathrm{iL}}$ is 0.375. ${ }^{20}$ Thus the smaller core, with the pesticide dealer, is slightly stronger than the core formed by NGO representatives and farmers; and thus is the dominant core in the knowledge network. A comparison of the composition and structure of the strongest two cores, and their connectedness to the rest of the network, can help in understanding the poor NPM innovation capacity of Punukula farmers.

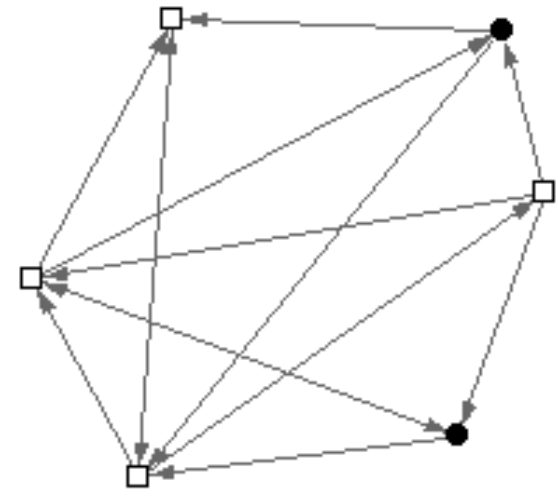

a)

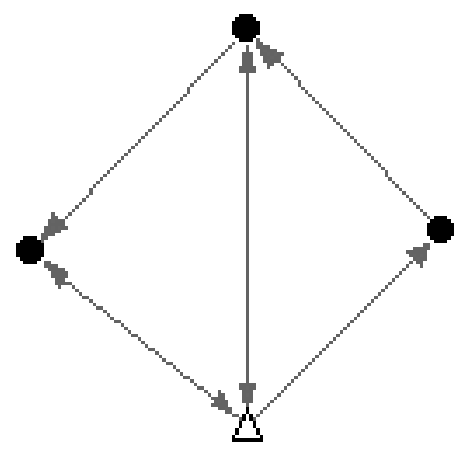

b)

Figures 6.4 The strongest two cores in the knowledge network. The unfilled node in (b) represents the pesticide dealer, and the unfilled nodes in (a) represent the local NGO representatives. All arrows represent knowledge flows.

\subsection{Discussion}

A stronger core implies an assured supply of (pesticide-related) advice if problems with the incumbent technology (pesticides) arise. The power of the pesticide dealer in the knowledge domain may be buttressed by his hold in the business domain (which is discussed in Chapter 7 on the business network). The supply of pesticide-related problem-solving knowledge gets reassured through embeddedness of these knowledge flows in business relations between the farmers and the pesticide dealers. This analysis of the interpenetration of the business and knowledge networks is presented in Chapter 9. A stable availability of advice in the new technology is less certain due to the weaker core, of Figure 6.4a, structured around the NGO representatives. Furthermore, the NGO representatives (or any other NPM actors for that matter, the business network for NPM is practically non-existent) do not have a significant amount of business ties with the farmers.

One must however be cautious here: the structural strength of two small cores may not tell the whole story of wider innovation capacity in the village. A core must be connected to the rest of the network and its distance to others in the network

${ }^{20}$ Note that, in both Figures, some edges have arrows on both ends, which implies two (directed) internal links in the core. 
(particularly the farmers) determines its influence on the wider innovation capacity. For example, the wider influence of the dominant core in the knowledge network may lower the costs of switching back to pesticides after the use of NPM for one season, which can help explain the deficit in the third component of innovation capacity i.e., sustainability of NPM adoption. The ratio of farmers to non-farmers in the dominant core (b) is 3 as compared to 0.5 in the NGO-led core (a). ${ }^{21}$ Higher participation by farmers in core (b) allows the pesticide dealer better access to farmers, which promotes the establishment of stable knowledge exchange channels between him and the farmers. The higher participation of farmers in the pesticide-related knowledge core may have a wider 'social' dimension. The greater participation by farmers in core (b), through their social ties of kinship and friendship, may allow the pesticide dealer to buttress his power in the knowledge domain with greater access to farmers through the kinship/friendship domain. This embeddedness in friendship ties in turn may improve his ability to offer suitable problem-solving advice to farmers.

The influence of the dominant core is confirmed through a quick examination of how well the two cores shown in Figure 6.4 are connected to the rest of the knowledge network. The pesticide dealer, who is the central member in core (b) with clearly the highest out degree centrality, as shown in Table 6.3, has the best reachability to other nodes in the network. Through his outgoing links, he can reach (directly and indirectly) 119 out of a total of 155 Punukula farmers. By comparison the most connected NGO representative is only able to reach 67 Punukula farmers. ${ }^{22}$ This makes core (b) better connected than core (a) to the rest of the knowledge network.

Third, the stronger core has a higher number of bidirectional knowledge flows between farmers and non-farmer(s) (two in core b as compared to one in core a). These two-way flows are essential for joint problem-solving and co-development of a technology. This lack of two-way flows between farmers and 'experts' has implications for the second dimension of innovation capacity concerning farmer experimentation and sharing of experiment-results with 'experts'. I observed in section 6.3 above that channels for transfer of new knowledge from farmers to NGO representatives were rare. This observation is confirmed by the structure of core (a). Greater participation of farmers in knowledge sharing, which is essential for building innovation capacity, would have involved a larger number of two-way knowledge flows (and strong cyclic cores) among the farmers, and between the farmers and NGO representatives.

This lack of bidirectional flows between farmers and NGO representatives is a wider feature of Punukula's knowledge network. Farmers for the most part played the role of passive knowledge recipients rather than active innovators and knowledge sharers in the network. Also, there is only one farmer-to-farmer bidirectional exchange

21 The innovative dynamism of the dominant core (b) in the knowledge network is largely due to the pesticide dealer and three farmers. The other core (a) is formed by four NGO representatives and two farmers.

22 The average distance of the all-in-one dealer and the NGO representatives to their respective reachable Punukula farmers is approximately equal (1.9 and 1.94 respectively). 
in the knowledge network as a whole (which as mentioned earlier precludes any possibilities of formation of a strong core constituted by farmers alone). Thus, the arguments about lack of farmers' participation in core (a) can be forcefully applied to the entire knowledge network in which there are few two-way knowledge exchanges between farmers and NGO representatives, and underline the low direct involvement of farmers (and their knowledge) in 'participatory' development beyond their role as recipients of problem-solving advice. 


\section{The farmers' escape from pesticides and debt}

The aim of this short chapter is to analyse the second aspect of the successful experience with NPM in Punukula, as highlighted by the media and NGO reports. Here I examine the extent to which NPM had released Punukula farmers from the grip of local all-in-one dealers and thus from escalating debt. The analysis is performed using data on the business network for agricultural inputs and credit. As in the previous chapter, I use concepts developed in part I of this dissertation, namely the core-periphery distinction and circular process, to analyse network structure.

The chapter is structured as follows. I start by describing the business network structure. Following this, I identify the dominant core in the network. I end the chapter with a brief discussion.

\section{I The business network: accessing agricultural inputs}

The business network of Punukula farmers deals with two steps of the agricultural production chain. These two steps include a forward linkage of the farmers to buyers of their crop, and a backward linkage to the farmers' inputs suppliers. The inputs considered are seeds, pesticides, fertilisers, NPM materials, and credit. The outputs considered are cotton, rice and pigeon pea. The crop buyers are generally middlemen and traders who buy the crop locally from the farmers and sell it in larger markets such as at the market-yard for agricultural produce in Warangal district or in the district headquarters of Khammam. All connections of Punukula farmers through the forward and backward linkages are combined to form the complete business network of the village.

The combined business network of Punukula farmers is shown in Figure 7.1. Punukula residents are represented as black nodes and people/organizations from outside the village are shown as unfilled nodes. The black-node isolates in the Figure are Punukula residents who are not farmers (they are either the landless farm-labourers or village shopkeepers). As shown in the Figure, most business transactions of the farmers are with people from outside the village. Further, a large part of the network exhibits a star or, more accurately a floral structure with a local all-in-one dealer occupying the most prominent position at the centre (with the largest number of incoming and outgoing links). This implies that a large percentage of farmers procure one of the farm-inputs or receive credit from this dealer and sell their crops back to him. See Figures 7.2 and 7.3 for diagrams of the farmers' input-side (including credit) and output-side business networks respectively. By letting the farmers procure farminputs on credit, or by providing direct loans at high interest rates, the dealer gains control of the farmers' production process (note the dealer's position at the centre of the farm-input network, as shown in Figure 7.2). On the output side, when the dealer buys the farmers' crop, he buttresses the control gained through credit-provision (see Figure 7.3). This combination of mutually-reinforcing input and output business relations of the farmers with the all-in-one dealer forms a circular process. 


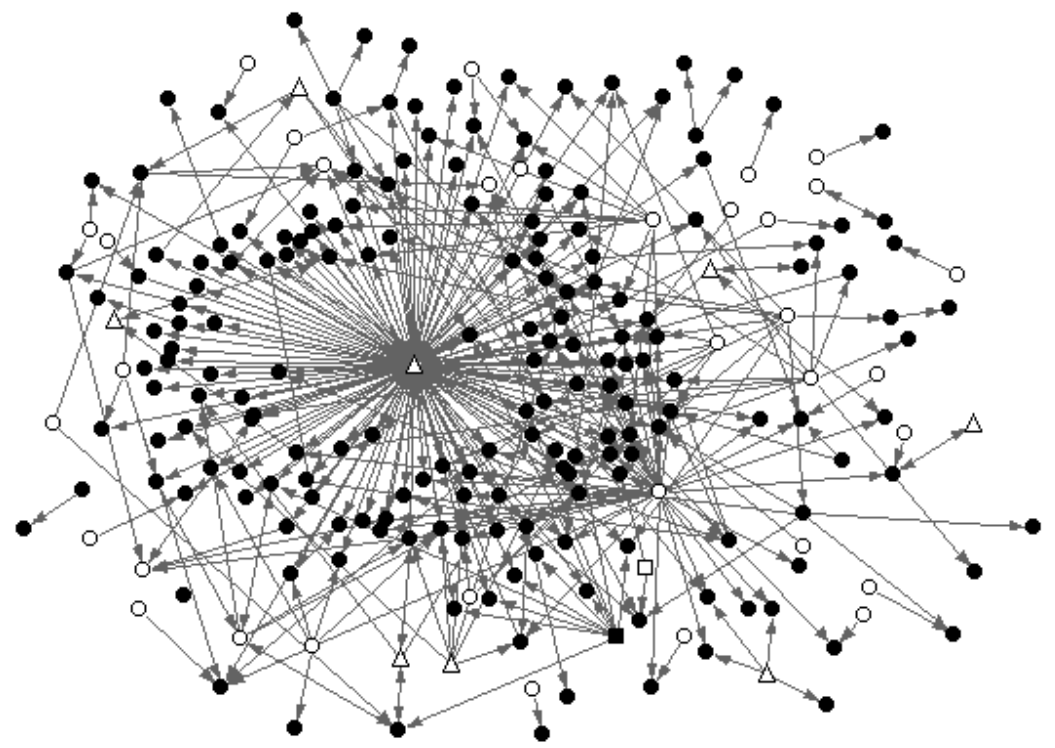

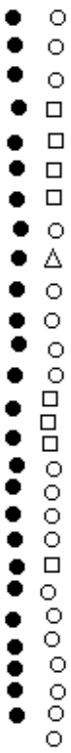

Figure 7.1 Punukula's combined business network for credit, crop sale, and farm-inputs. Filled nodes represent Punukula residents whereas unfilled nodes depict people from outside the village. Nodes representing all-in-one dealers are triangles and NGO representatives are squares. Arrows represent flow of farm-inputs, or credit, or the farmers' crop. Nodes on the right of the Figure are isolates.
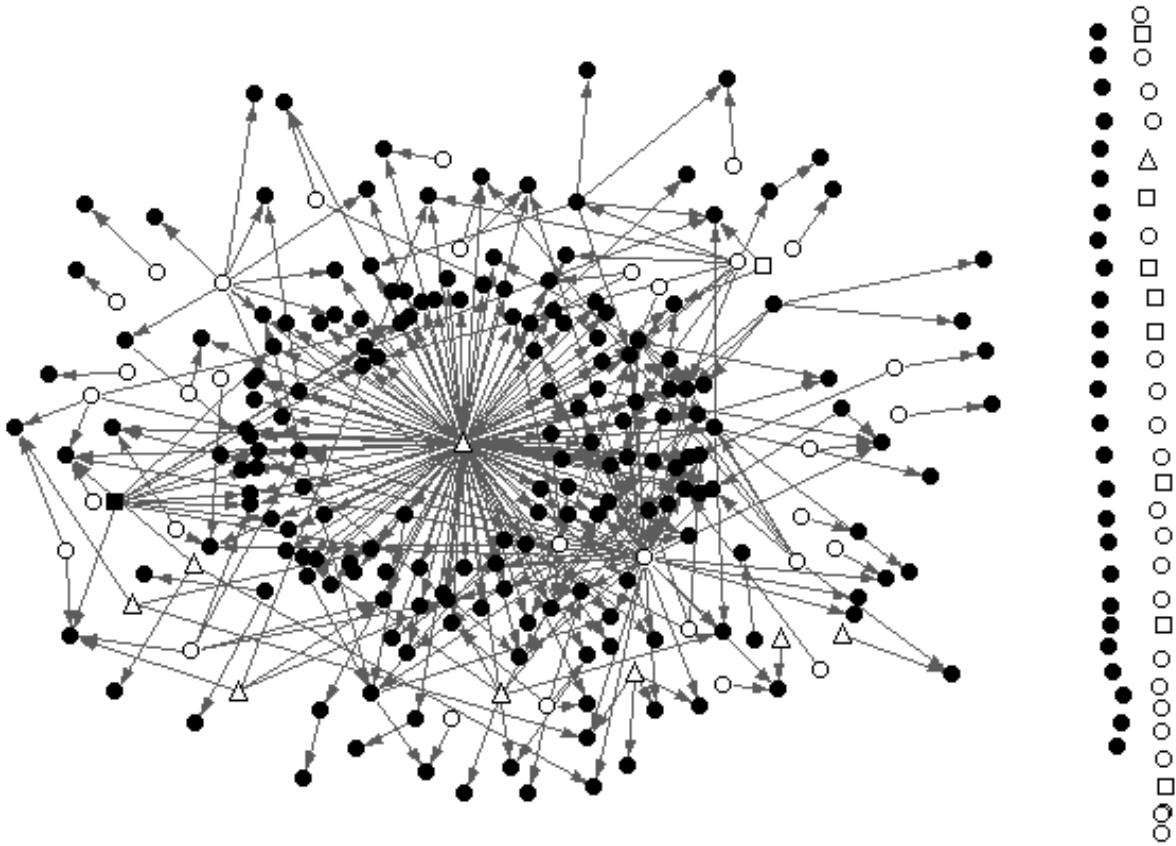

Figure 7.2 The farmers' network for procuring farm-inputs and credit. Arrows show the flow of materials from the all-in-one dealers to the farmers. Nodes on the right of the Figure are isolates. 

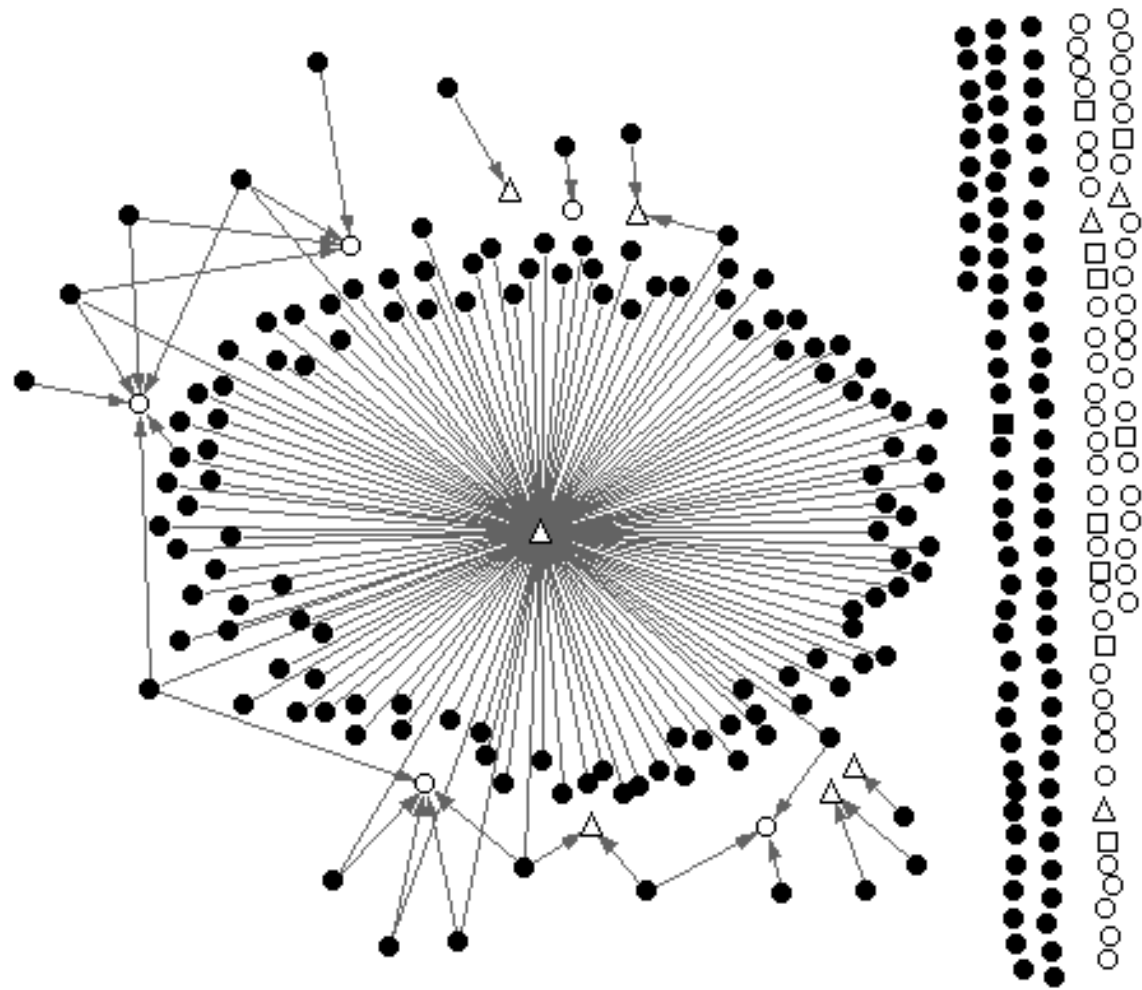

Figure 7.3 The farmers' network for selling their crop. Arrows show the flow of crop from farmers to buyers. Nodes lined on the right of the Figure are isolates. The number of isolates is greater in this figure because of the exclusion of the landless who were members of the credit network in Figures 7.1 and 7.2 .

\subsection{The dominant core in the business network}

I have identified 173 circular processes (cycles) in the business network of Punukula. 161 of these cycles involve the main supplier of farm-inputs to Punukula, M. Raj. As was the case with the knowledge network, all the circular processes have at least one of their constituent members from outside the village. However, unlike the knowledge network, none of the cycles in the business network involve a NGO representative.

Most cycles are of size 2: formed by a farm-inputs dealer and a farmer who buys farm-inputs, or takes credit, and sells crop back to the same dealer. From the set of cycles of size 3 or more, I define the dominant core by calculating the density of internal links using equation 6.1a. The dominant core is constituted by two all-in-one dealers and two farmers from Punukula (with $\mathrm{d}_{\mathrm{iL}}$ equal to 0.375 , the same strength as the strongest core in the knowledge network, see Figure 7.4 below). The all-in-one 
dealers who are members of the core are the main farm-input supplier, M. Raj, and a second supplier of farm-inputs to the village, Q. Ramani. The two farmers join the core simply because they buy from, and sell to, more than one all-in-one dealer (so they are not as dependent, on one dealer, as other farmers in the village who go to the same dealer for all their forward and backward linkage transactions).

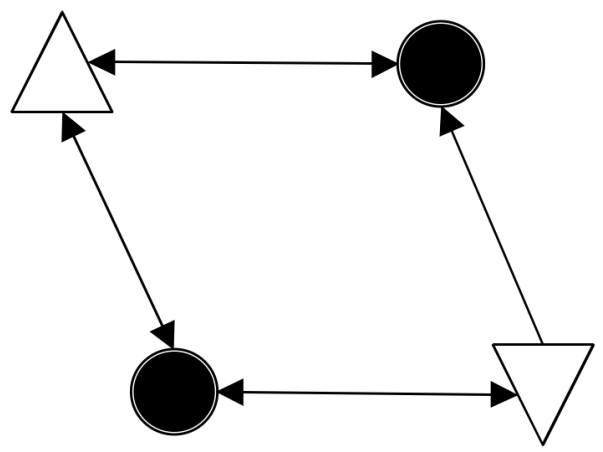

Figure 7.4 Structure of the dominant core in the business network. Triangles represent the two pesticide dealers and the circles Punukula farmers.

Finally, it is clear from the constitution of the cycles in the business network that the business network is dominated by the farm-input/credit provider to Punukula: this person, who also acts as the main crop buyer from the village, is a member of 161 of the total 173 cycles. Not surprisingly, he is a member of the dominant core of the business network, possessing two incoming and two outgoing links to other members of the core (as shown in Figure 7.4). The dependence of the farmers on the farm-input supplier, credit-provider and crop-buyer can be partly explained by historical factors related to the business transactions of smallholder farmers with non-farm actors (cf. Robert 1983 on historical aspects of this dependence and control achieved through credit provision; also see Amin 1982 for a historical account of the dependence of sugarcane cultivators through their forward linkages in North India). In addition, the dependence on the farm-input dealers is related to the green revolution of the $1960 \mathrm{~s}$ when input-intensive agriculture was promoted by the provincial and national governments in India. ${ }^{1}$

${ }^{1}$ During the green revolution, the government agricultural extension services provided material and knowledge support to resource rich farmers (with large irrigated landholdings) across AP to encourage them to adopt chemical inputs (fertilisers and pesticides), and high-yielding seed varieties (Wade 1988). Poor farmers in the villages tried to copy the rich farmers by observing (for example, Punukula farmers copied the migrant farmers from Guntur in growing cotton and using pesticides for the first time, cf. Vasavi 1999 on a similar pro-rich bias of other government-run programs). By the mid 1970s, once the farmers had adopted the new technologies, extension services all but withdrew from the fields. Farmers were left without advice and without the capability to experiment for solving their own problems (the focus of the green revolution was on effective and widespread adoption of the three complementary technologies, rather than capacity building for farmer-led 


\section{7·3 Discussion}

In the foregoing, I have argued that the farmers of Punukula are dependent on the all-in-one dealers due to their control over both the backward and forward business linkages of the farmers. This dependence and control is evidenced unequivocally through the centrality of the main farm-input supplier to the village in the business network (as clearly depicted in Figures 7.1-7.3). The high centrality of the dealer translates to him being a member of the dominant core in the business network.

The critical point here however is the unchallenged dominance in the business network of Punukula by the all-in-one dealer and the strongest core he is a member of (see Figure 7.4 above). The NGO-led project to wean the farmers off the all-in-one dealers, and the concomitant problems of heavy pesticide-use and ever-increasing debt, was largely unsuccessful. ${ }^{2}$ The business network structure shows that a strong core, either made up of farmers and NGO representatives or a group of farmers, did not emerge in Punukula's business network. ${ }^{3}$ Recalling the simulation results from part I (chapter 3) of this thesis, this competing core (of strength equal to or more than the existing dominant core) is necessary to dislodge the dependence of the periphery on an existing dominant core. The necessary condition for the emergence of such a competing core in the business network is the establishment of a local economy for the sale and purchase of NPM materials in or around Punukula. In this respect, the efforts of the local NGO representatives and the NPM farmers did not succeed. And therefore, the promise of a local (village) economy for NPM materials was not realised.

innovation). The lacunae left behind by the exit of extension services were gradually filled by private farm-input dealers which mushroomed all over rural and semi-urban areas in the 1970s and 80s.

2 All other cycles in the business network of size greater than 2 involve at least one all-in-one dealer. Thus, even a weak core in which the links represent exchange of NPM materials did not exist.

3 The links of this strong core, if it existed, would have represented the trade of NPM materials among farmers, farm-labourers, local shopkeepers, and/or NGO representatives. 


\section{Socio-political events and social network structure}

The aim of this chapter is to explain the socio-political activities surrounding NPM in Punukula. In particular, these activities concern the village's media success and the 'pesticide-free' resolution of the gram panchayat. The structure of Punukula's social network forms the basis of my arguments here. The socio-political events in the village follow two patterns. First, a considerable degree of group action facilitated the villagers' collective liaising with the local NGO and later led to the projection of a pesticide-free face of the village to the journalists. This group action eventually brought about the adoption of the no-pesticide resolution by the gram panchayat. The second pertains to a later factional divide in the village that became apparent during the visit by the AP minister of agriculture.

Unlike the networks studied in the previous two chapters, all links in the social network are symmetrical or undirected. As a result, circular flow takes place between any two actors, or all dyads in the social network are circular processes. Thus I use a slightly different strategy, than that used in Chapters 6 and 7, to locate the cores here. First I employ two structural measures of power and social capital due to an actor's position in the social network to identify influential members in Punukula's social network. ${ }^{1}$ Second, I check if each member of this elite group is connected to at least two other members. This check ensures that the network structure of the sub-graph formed by the elite group is a symmetric circular process (or a cycle in which nodes are connected through undirected links). Subsequently, I discuss how the core-members placed themselves between the NGO representatives and the rest of the villagers to bring about the panchayat resolution and achieve the media publicity.

The chapter is structured as follows. I start with a review of anthropological studies on the (multi-caste) Indian village and connect it back to Punukula's social network. The central purpose of the following section is to highlight the importance of interpersonal relations in a country where social situations have been generally explained by caste alone. This is followed by a brief review of the theory of collective action and the role played by community leaders in this process. A third section identifies suitable network-structural measures for power and social capital for the

${ }^{1}$ Here I am combining two structural (positional) measures to find powerful people in the village. These two measures are complementary. The first (closeness centrality) allows the identification of people who are easily contacted or reached by others in the network and have a high degree of independence. The second (network constraint) identifies people who have the potential to act as brokers between separate sub-networks. More details are presented later in the chapter where I briefly review the social network literature on network-structural aspects of power and social capital. Furthermore, these purely structural measures of power and social capital of a farmer can be compared to, and juxtaposed against, traditional socioeconomic measures such as landholding, access to irrigation facilities, and type of housing. Such a comparison is not attempted here and the focus on social network measures is maintained throughout the chapter. 
present purposes. And finally, the core-periphery structure of Punukula's social network is analyzed.

\section{I Village India and social networks}

Study of the Indian village was a popular enterprise among sociologists and anthropologists in post-independence India of 1950's to 1970's (for example, see the work of Srinivas 1955; 1959; 1975; Marriott 1955; Fordham Norr 1976; and Thorner 1954 for a review of some early studies). These social scientists believed village India to be a mirror of the larger Indian society most of which was agrarian then as now. This structural unity and character of the village is affirmed by Srinivas (1955 [2002:71]) while referring to the Mysore region in South India,

"Each village is a tight little community in which everyone is known to everyone else, and in which a great deal of experience is common to all. Agricultural activities in which the vast majority of the villagers are engaged impose the same activity upon all of them at any given period in the year."

Fifty years later, Punukula in the nearby region of Telangana in Andhra Pradesh possesses the same apparent characteristics as the Mysore villages.

The village studies have provided an in-depth understanding of the social structure of Indian villages in terms of relationships between different constituent social groups (castes). They have in general also highlighted the importance of links connecting the village to the outside world. However, I did not come across any studies that mapped the social networks of a village's inhabitants despite an important paper on networks in rural India by two prominent sociologists (Srinivas and Beteille 1964). ${ }^{2}$ This relative neglect of interpersonal networks of village India may be due to the influence of Dumont (1970) where he argued that the ideology of caste-based hierarchy is the only way to understand Indian society. ${ }^{3}$ In an earlier work, Dumont and Pocock (1957) affirmed that deep caste-based divisions make a local community in the form of a unitary village impossible (Srinivas 1975). Even the gram panchayat, according to Dumont, should not be viewed as a corporate organization of the village but that of

2 Another early study on networks in Indian society is due to Cohn and Marriott (1958). Their focus was much more general to be of direct relevance to the present analysis. They were interested in the administration of a large, heterogeneous nation such as India through coordination in social networks (Freeman 1979). In addition, I have come across one study that makes references to social networks in rural India, not so much as following the appeal of Srinivas and Beteille (1964) to trace intra- and extra-village links in the three institutional areas of economic exchange, political connections, and kinship relations, but using information from secondary sources to create general model-driven pictures of an Indian rural community (see Chapter 7 in Quigley 1993). However, none of these studies developed or used social network tools to perform structural analyses.

3 Dumont's (1970) study was at the same time severely criticised by many sociologists and anthropologists from different angles. The most systematic criticism, assessing Dumont's central dualism between purity and pollution and his englobing of everything in Indian society by caste-based hierarchy, that I have come across is due to Marriott (1969; 1976; 1998). Also see Habib (1995) for a historical, and Raheja (1988) for a village-study based, critique of Dumont. 
the dominant caste in the village (Wade 1988). ${ }^{4}$ Furthermore, in 'Dumontian orthodoxy', inter-caste (or sub-caste based inter-group) relations are sufficient for explaining the rural (primeval) Indian social structure (see Gell 1982: 485 for an exposition of this argument). Dumont considers all other ideologies including the one rewarding individuality as incompatible with the central ideology of caste-based hierarchy (Marriot 1969). As a result, individuals, and their potentially diverse intra/extra-village networks, are pushed into the background as inter-caste relations provide the only true insight on Indian society (Mines and Gourishankar 1990).

A second reason for the neglect of the study of networks in Indian villages may pertain to the rapid changes in the rural environment in the last few decades. If the reality of the Indian village as a tight little community was questionable fifty years ago, it is definitely non-existent now (see Gupta 2005 for a polemic along these lines). Changes in rural contexts have included political changes at the village level in the form of rules for panchayat's composition in multi-caste villages; a new model of agriculture, ushered in by the green revolution, which forces farmers to visit nearby towns for purchasing farm-inputs; various rural development projects that bring villagers into frequent contact with government and non-governmental organizations from outside the village; improved access (better roads and means of transportation) to the urban areas; and the diffusion of cable television. These changes may make studying a network whose boundary is the same as that of a village less valuable in understanding rural Indian society and its dynamics. In addition, the loosening of an individual's allegiance may not be restricted only to her village, but also to her subcaste and lineage (Srinivas and Beteille 1964). This fluidity in the boundary of different kinds of social groups implies that one must make individuals and their networks, rather than timeless groups such as castes and village, as subjects of direct analyses.

Beginning in the 1980's, an alternative understanding to caste-based hierarchy emerged among Indian ethno-sociologists and historians. Barring a few exceptions, this newer work does not focus on village studies, but at a more general level explores ways in which Indian society is organized by factors other than caste hierarchy. These studies, for example, highlight the critical distributive role played by centralised religious agencies in organizing Indian society (Mines and Gourishankar 1990).

"In post-colonial times, at the local level, these agencies have been the dominant castes (jajmaan) of the villages (Raheja 1988b) and the patrons of worship (Yajamaana) among urban temple congregations (Appadurai 1981)." (Mines and Gourishankar 1990: 765).

If the above emphasis on religious agencies is broadened, other agencies such as the gram panchayat and development (vikas) committees have played a similarly important role in reshaping the power structure of Indian rural society at the local-level (Oommen 1970). At higher levels, multiple development agencies in the government

4 The term 'dominant caste' was coined by Srinivas (1955; 1959). According to Srinivas (1955 [2002:57]), "A caste may be said to be 'dominant' when it preponderates numerically over the other castes, and when it also wields preponderant economic and political power. A large and powerful caste group can dominate more easily if its position in the local caste hierarchy is not too low." 
and non-governmental spheres have surfaced which also affect social relations at the village level. For example, the partial land reforms carried out by the government have shaken the traditional caste-based hierarchy in some villages (see Mendelsohn 1993 for a case study). In some regional contexts and castes, a long history of individualism and family-centredness also acts as a hindrance to the dominant caste-derived hierarchy in villages (Mendelsohn 1993). To summarize, the later trend in sociology and anthropology highlights that social relations in India may be determined by "dominant groups and persons more salient than caste-hierarchy" (Mines and Gourishankar 1990: 765).

As I demonstrate later in the chapter, interpersonal social network analysis is an appropriate way to capture this dominance of persons and groups beyond the unchanging caste-hierarchy. The existence and identification of groups is then not simply synonymous with the individual's sub-caste derived group. Using social network maps, groups constituted by individuals from multiple castes can be identified as easily as those traditional groups limited to an individual's caste or lineage. According to Srinivas and Beteille (1964:166),

"Networks can be either close-knit or loose-knit. In other words, the chain of reaction emanating from a person may either lead back to him, or it may not. In traditional India, particularly in the South, the network of kinship and affinal relations was a close-knit one. In fact, this network had, inevitably, to stop short at the boundary of the sub-caste, and therefore to form a closed circuit." 5

As a simple test of this importance of traditional sub-caste and lineage driven groups, ${ }^{6}$ Punukula's social network (strong ties of kinship and friendship) is shown as a graph in Figure 8.1, where each caste in the village is represented using a different shape. Vertices of the graph representing members of the network who are not Punukula inhabitants are unfilled. Note that despite the fact that kinship ties by definition are intra-caste, so all inter-caste ties in the Figure are friendship ties among the villagers.

5 The same argument of closed circuit within cohesive groups, in a different context, was used by Coleman (1988) in a much-celebrated exposition of social capital due to 'closure' in networks. Also note the similarity to the circular process as elaborated in the theoretical framework of previous chapters of this thesis.

${ }^{6}$ The test done here is purely a graphical representation. I do not use any mathematical techniques to identify cliques in this network as caste or its loosening grip on Indian society is not the focus of the present discussion. 


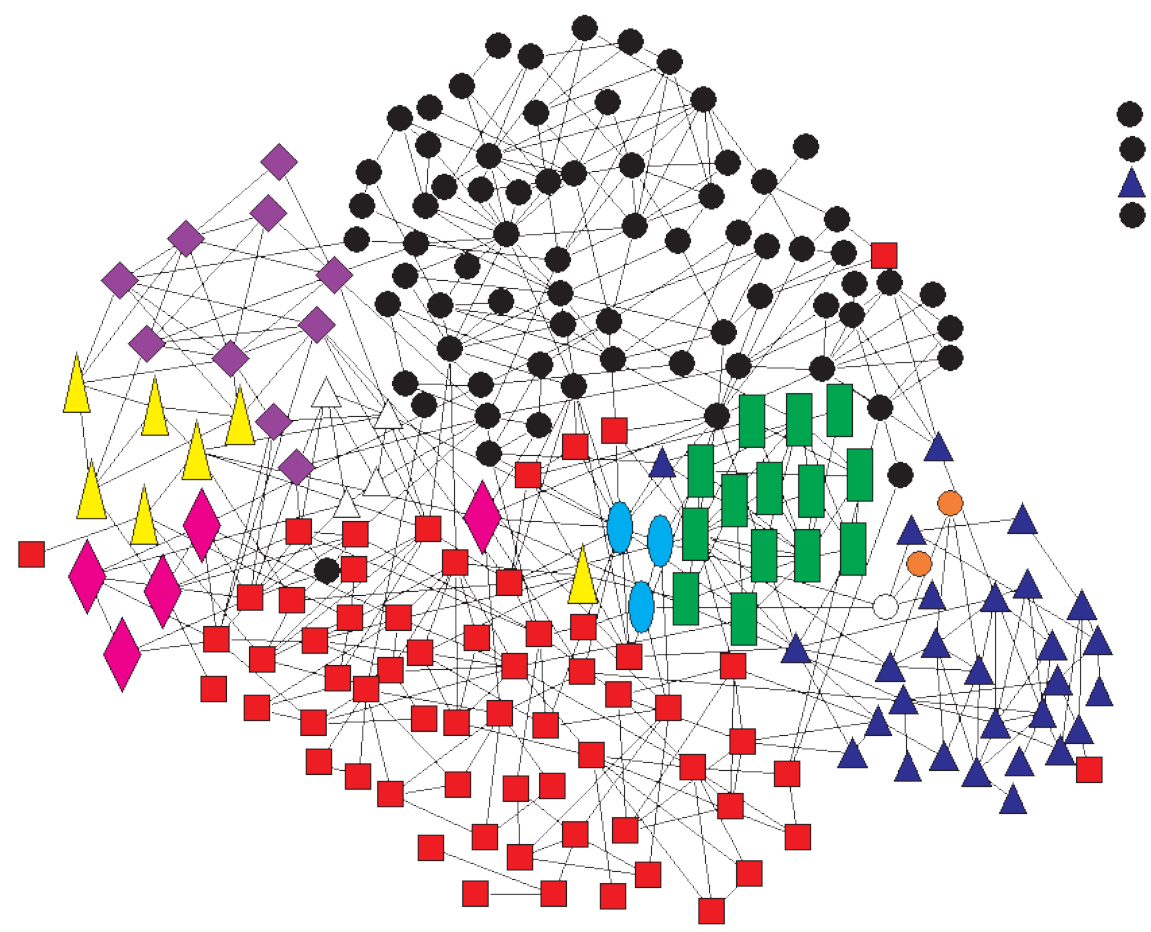

Figure 8.1 Caste-based distribution in Punukula's social network: each shape represents members of one caste. Only the inhabitants of the village are shown. There are four isolates, drawn in the upper right hand corner.

In Figure 8.1, we observe a preponderance of intra-caste links (there are a total of 737 within-caste links as opposed to only 248 inter-caste links - a ratio of 3 to 1$).{ }^{7}$ But, while it is clear that while caste remains important and observable, many individuals cut across caste (and sub-caste or lineage) boundaries to form strong friendship ties with people of other castes. ${ }^{8}$ This observation is consistent with the later literature on the loosening of caste-based hierarchy as a result of newer organizations and associations in village India.

\footnotetext{
7 These calculations are based on the data on caste available for 210 households in the village. The presence of at least five small-sized caste groupings (see Table 4.4 below), whose members have a significant proportion of their social ties with members of other castes, raises the total number of inter-caste links in the village. In addition, the second largest caste group of Yadavas in the village has a significant proportion of their ties with other castes, which reflects the village-level ratio, 74 out of a total of 286. For members of the biggest caste, Koya, only 40 of their links are with other castes as opposed to 300 within-caste links - a ratio of 7.5.

8 Note that the social network of Punukula I mapped is restricted to strong social ties only. A network that included weak ties (defined as casual contacts or friends-of-friends) would definitely look different and would lead to a reduction in the preponderance of intra-caste links.
} 
In addition, the smaller the caste-group the more cliquish it is (for example, consider the Mala's depicted as green round nodes in Figure 8.1). However, members of very small groups (such as the three Choudhary households drawn in cyan and the four Dudekula's depicted as brown nodes) tend to be more integrated in the larger network than the Mala's or the Mudiraj's (shown as purple trapezoidal nodes in Figure 8.1), both of which have 10 or more households in the village. ${ }^{9}$ The bigger caste groups seem to be more scattered, and divided into multiple smaller groups which are derived largely based on lineages or extended-family groupings. For example, the Koya's (black round nodes) in Punukula are divided into three big lineages, two of which are more clearly cliquish in the graph than the third. One or two lineage-based cliques among the Yadava's (red square nodes) are also clearly visible.

Punukula has 10 social groups. I have caste information for 210 out of a total 212 households (see Table 8.1). The remaining two households operate small shops in the village and do not own any land. The largest group of people in Punukula are those belonging to the Koya tribe. Not far behind in number is the group of people belonging to the peasant caste, Yadava. The third and fourth largest groups are the Lambadi (blue triangular nodes in Figure 8.1) and the Dalit Mala respectively. ${ }^{10}$ No single social group dominated clearly in numbers: the difference between the two largest groups is only 13. Neither was there a domination of one group in economic power as measured by total land area owned or access to external resources: the Koya's collectively own 61.8 hectares and the Yadava's own 62 (as displayed in column 3 of Table 8.1). ${ }^{11}$ Therefore, using Srinivas' (1955) criteria, I can easily conclude that Punukula does not have a single dominant caste. ${ }^{12}$

\footnotetext{
9 The Mala's are even more strongly cliquish than the Mudiraj's because all except one Mala households in Punukula belong to a single lineage (Kasimalla).

${ }^{10} \mathrm{Dalit}$ is a term used to refer to people belonging to the untouchable castes.

${ }^{11}$ I only use the amount of land owned as a measure of economic power to identify the dominant caste in Punukula, as defined by Srinivas (1955). Other more ambiguous measures such as the availability of irrigation (most land in Punukula is either rain-fed or bore-well irrigated) and type of house (concrete-slab roof house versus a thatched hut) can be used as well. However, using these measures does not change the gist of the argument here that there is no dominant caste in Punukula. This fact was confirmed in my interviews with the villagers. Nearly everyone I spoke to maintained that there is no caste divide, or oppression, in Punukula. Finally, I collected the data on land owned using the survey questionnaire based interviews, whereas the other data was sourced from official government and NGO records. This official data was incorrect for a number of categories including the total number of households in the village which was put at 189: another reason why I relied on my own data only.

12 The two largest castes if combined are definitely dominant in the village. Together they form about two-thirds of the population and own more than $60 \%$ of the land. But, the two castes cannot be reasonably considered as one large group because of the small number of links that connect them together (the koyas have very few inter-caste links in general, see footnote 7 above).
} 
Table 8.1 Caste and land distribution in Punukula

\begin{tabular}{|c|c|c|c|}
\hline Caste & $\begin{array}{l}\text { Number of } \\
\text { households }\end{array}$ & $\begin{array}{l}\text { Total land owned } \\
\text { (ha) }\end{array}$ & Shape in Fig. 8.1 \\
\hline Koya & 75 & 61.8 & Round \\
\hline Yadava & 62 & 62 & Square box \\
\hline Lambadi & 29 & 21.4 & Triangle (small) \\
\hline Mala & 14 & 19 & Rectangle \\
\hline Mudiraj & 10 & 20.2 & Diamond \\
\hline Potter/Carpenter & 7 & 5 & Triangle (long) \\
\hline Goud & 5 & 3.2 & Diamond (long) \\
\hline Dudekula & 4 & 2.4 & Triangle (unfilled) \\
\hline Choudhary & 3 & 1.2 & Ellipse \\
\hline Reddy & 1 & 0 & Round \\
\hline
\end{tabular}

Finally, to summarize the foregoing, a dominant caste, driving a strictly hierarchical structure of inter-caste relations, is not observed in Punukula. This may be due to the large percentage of tribal population in the village. Tribal societies in India are generally believed to be less hierarchical than the caste-Hindu social systems (Oommen 1970). In addition, some other castes in the village, such as Yadava, Mudiraj and Goud are accorded the same (backward) caste status by the government. The lack of caste-based hierarchy, and oppression, in Punukula was also affirmed by almost everyone I spoke to in the village during the fieldwork. SECURE representatives, who worked with farmers from all castes in the village, had the same opinion about Punukula's social structure. In addition, as depicted in the graph of Punukula's social network in Figure 8.1, a large number of bridging inter-caste friendship ties exist in Punukula despite the preponderance of social (kinship and friendship) ties among members of the same caste and lineage. ${ }^{13}$ Therefore, a dominant caste, and caste-based hierarchy, cannot be viewed as the driver of collective action in the village. Alternate explanations must be found through identification of individuals or new social groupings that drove the collective action and media success in Punukula.

\subsection{Collective action and media success}

The purpose of this section is to understand the roots of the collective action in Punukula that led to the adoption of the panchayat resolution, and the successful projection of a pesticide-free image by the farmers to the media. This discussion is based on the premise that both 'successes' in Punukula required group action by its inhabitants.

13 This cliquishness in Punukula's social network must be confirmed using a decomposition of the network using computational techniques. 
There are two commonly used social scientific explanations for group action. The first explanation is based on rational choice, sometimes called the pessimistic theory of collective action (Wade 1988; Gould 1993). Here self-interested individuals do not act collectively in common interest unless coerced or compelled to do so (Olson 1971). This coercion can either be provided in the form of material benefits or as punishments to those members of the group who do not participate. Further, high external costs due to severe ecological risk or scarcity may also act as impetus to group action. But when such internal or external coercion mechanisms do not exist, collective action does not take place.

The second intellectual tradition for studying collective action departs from the atomistic rational self-interested individual in order to seek explanations in the social structure of a group. Here one actor's contribution depends upon the contribution of other socially related actors. An actor is either driven to contribute by norms of fairness toward friends or due to hierarchical persuasion by community leaders or the state. This way of thinking has been more popular among sociologists and social anthropologists (see for example, Walton 1992; Gould 1993). In the present discussion on Punukula, I largely adhere to this second tradition, while building on some insights from the first. In particular, I focus on the social network of the village to understand the structural drivers of group action. I use data on the social network structure of the village, employing (structural) interpretations of power and social capital from studies in social network analysis in combination with circular process driven core-periphery structures. I also briefly discuss some socioeconomic characteristics of a core group of individuals who acted as leaders in persuading others in the village to act collectively.

Unlike many studies on group action, the focus of the present discussion is not to study the level of participation of group members in collective action, or analyse the outcomes/functions served by it. My analysis is restricted to social structural aspects of the village. Other possible drivers of group action such as ecological risks, scarcity or the promise of material benefits are not studied here. The latter formed the core of an extensive study of collective action in 31 villages in Andhra Pradesh by Wade (1988). ${ }^{14}$

The Punukula gram panchayat has members belonging to at least three other neighbouring villages, these villages are smaller than Punukula as is their representation in the panchayat. ${ }^{15}$ Four of the ten members of the panchayat, including the president, are from Punukula. Punukula thus is the central village having control over the gram panchayat, although being part of a bigger conglomeration of villages limits the power

\footnotetext{
${ }^{14}$ Wade studied collective action of rural communities to manage public goods in agriculture such as water and the crop/livestock-grazing nexus.

15 The gram panchayat is the village council with elected members. The panchayat is the main form of local government across rural India. Its responsibilities include a variety of local development activities such as maintaining village roads, building small irrigation facilities, etc. (Jha 2004). A large village of around 1000 households may have its own panchayat whereas smaller villages are generally clubbed with 3-4 other neighbouring villages under one village council. Caste-based reservation of seats in the council ensures that all (major) castes in the villages are adequately represented.
} 
of Punukula inhabitants in getting the panchayat to adopt resolutions. A central claim I make in this chapter is that collective action underlying the panchayat resolution and the media publicity was led by a group of core inhabitants of Punukula.

\subsection{Power, influence, centrality, and brokerage}

If we recall the media story of Punukula's success from chapter 4, two sociopolitical activities of the villagers come to the fore. First, the projection of the face of success to the media reporters. Using the core-periphery analogy, I argue that the influence or 'hold' of a core group of people in the village created the projected face of unity to the outside world (until the agriculture minister's visit). Second, the media fame, the panchayat resolution, and the actual (partial) success with NPM would not have been possible if the villagers had not liaised effectively with the local NGO. Here I argue that a core group of people from the village acted as brokers who linked the farmers with the NGO representatives. This brokerage was also required to bridge the different sub-caste based groups in the village. ${ }^{16}$ Therefore, in the following, I focus on identifying a group of influential people in Punukula using a common measure of network centrality. Subsequently, I discuss ways to identify people who are strategically located in the social network to play the role of brokers.

\subsubsection{Power and influence in a social network}

The study of power structure of communities has a history of more than 50 years: following the pioneering work of Hunter (1953), intensive research from the 1950's to the 1970's made the study of community power an important sub-field of sociology and political science (Walton 1976). The literature in this stream is therefore vast and this sub-section is not a review. I only present a brief overview of some main strands of the literature to set the stage for a discussion on structural measures of power in social networks. Researchers in the first power-structure studies used four broad types of methodologies (Walton 1966):

- the reputational method, first carried out by Hunter (1953), where members of a community are asked to identify influential people;

- the positional method which considers people holding influential positions in community organizations as leaders;

- the decision-making approach in which leaders are people who are instrumental in resolving a specific issue;

- $\quad$ and finally, the case study approach.

A few studies combined two or more of the above approaches (Walton 1966; Oommen 1970). Synthesizing insights from studies using the different methodologies, Oommen (1970) developed the concept of power pool.

16 Bridging between the different sub-caste groups in the village was important for the projection of the face of uniform village-wide success and for distribution of any resources accessed through the NGO in the village. 
"The power pool consists of (a) those who perform power-exercising roles or those who occupy formal power positions; (b) those who are considered to be leaders by the community; (c) those who are found to be exerting influence in the decisional process; and (d) those who are capable of preventing the implementation of decisions. Admittedly, there will be ample overlapping between these categories." (Oommen 1970: 228).

This concept of power pool is similar to the idea of the core group in Punukula's social network presented here. As is clear in the foregoing, barring a few sociometric analyses, the community power structure studies in sociology and political science use measures distinct from the structural ones used in Social Network Analysis (SNA) to identify community elites. In addition, most of this research studied communities only in the United States (Oommen 1970).

Social anthropologists on the other hand have worked with SNA from the 1950's onwards. However, their concern was largely restricted to simple measures such as network density (see Mitchell 1974 for a review). By the mid-1970's, SNA had begun to appeal to a group of sociologists interested in community power, led by Edward Laumann and colleagues who studied structure of power within community elites in small urban areas (see Laumann and Pappi 1976; Laumann et al. 1977). The work done by Laumann and his colleagues combines the earlier focus on reputation and issuebased decision making with a social network-derived positional approach. Another reason for the increasing attention to SNA may have been related to the various pronouncements that communities, as we traditionally knew them, were dead (see the studies in Wellman and Berkowitz's 1988 for the argument that 'liberated' networks have taken the place of closed communities).

Power and influence have been commonly viewed as tied to centrality in social networks (Gould 1990). Two main categories of centrality measures exist in the literature: first, those used to measure the centrality of individual nodes on the basis of their position in the network; and second, those measuring the degree of centralisation of a network as a whole (see Freeman 1979 for a review of nine such measures; and Wasserman and Faust 1994). According to Freeman (1979), the first measures of centrality came out of research done at the Group Networks Laboratory at MIT in 1940s. At that time, and later until the 1970s, research on centrality was largely focussed on communication networks and their efficiency. Maintaining this emphasis on efficiency and control of communication, Freeman (1979: 221-225) discussed three measures of node centrality in social networks: degree centrality, signifying the extent of a node's direct link with others, or its importance as a focal point in the communication activity in the network; betweenness centrality, the strategic location of a node on paths connecting other nodes signifying the node's potential for control of communication activity or as a "coordinator of group processes"; and lastly, closeness centrality, measuring a node's closeness to all other points in a network. ${ }^{17} \mathrm{~A}$ high closeness centrality of a node (small geodesic distances to all other nodes in the

${ }^{17}$ For formal definitions, including equations, of the three measures of centrality, see Appendix B of the thesis. 
network) implies that communication through this node can be done at minimum cost or time. It also implies that the influence of an individual with high closeness centrality spreads faster, and more easily, in a network. This measure applies to connected graphs i.e., networks without any isolates (Freeman 1979).

Bonacich (1987) has proposed other, more complex, measures of centrality where an individual's centrality depends on the centrality of others she is connected to. Here others' centrality can have a negative or positive impact on an individual's centrality. The positive, mutually-reinforcing, impact of individuals' centralities is akin to the positive feedback of one node's dynamism on other nodes in the cyclic core, as discussed in Chapter 3. This measure of centrality that accounts for the positive effect of the neighbours' connectedness is “a closeness measure of centrality in Freeman's (1979) sense; it is large when paths connecting it to other positions are the highly weighted short paths.” (Bonacich 1987: 1174). Therefore, I simply use the closeness centrality measure to find influential people in Punukula. Closeness centrality, for the present purposes, signifies perceived influence and power of an individual gained through ease of contact, reachability (access), and independence (Freeman 1979; Irwin and Hughes 1992). The first two aspects of the potential to access and contact others in a network are obvious through the emphasis on short paths. As pointed out earlier, these two aspects allow the influence of an individual with high closeness centrality to spread more easily through the entire network. The third aspect of independence underlines the appropriateness of closeness centrality for the present purpose:

"in this sense closeness centrality measures dominance and subdominance in a system. On the one hand, a point [or node] that optimizes direct and indirect access to the entire network is not dependent on other vertices, and hence occupies a dominant position. Peripheral points, on the other hand, must rely on these central points for access to the general network and, therefore, are dependent on these most central points." (Irwin and Hughes 1992: 20).

Therefore, the high degree of independence associated with highly (closeness) central nodes make them the core nodes on whose dynamism the peripheral nodes are dependent. Note that this distinction between core and peripheral nodes is equivalent to the core-periphery dependence observed in the model presented in the Chapter 3. The set of ten most 'dominant' or dynamic individuals and their closeness centrality values are listed in Table 8.2 and depicted as larger nodes in Figure 8.2 below. ${ }^{18}$ Precise definition and mathematical description of closeness centrality as compared to other forms of centrality are given in Appendix B. The software used for calculating the centrality index is Pajek (Batagelj and Mrvar 2007).

18 The size of this set of powerful people is kept small largely for purposes of presentation. The small size facilitates a clear depiction of the structure of social ties among these powerful people, as the dominant core in Punukula's social network. An example of a larger core constituted by people with the 15 highest (lowest) centrality (constraint) values is presented in Appendix C. The argument regarding the composition of the dominant core in Punukula largely remains the same with this core. 
Table 8.2 The set of ten most central individuals in Punukula

\begin{tabular}{|l|l|}
\hline Name & Closeness centrality \\
\hline Gorinta, EG & 0.260833 \\
\hline Veena, IG & 0.250732 \\
\hline Velu, HE & 0.244312 \\
\hline Moti, CQ & 0.242676 \\
\hline Koli, ED & 0.240423 \\
\hline Pillai, GB & 0.239155 \\
\hline Pai, DJ & 0.233909 \\
\hline Joardar, GX & 0.233307 \\
\hline Pillai, GA & 0.232708 \\
\hline Yarla, FQ & 0.231223 \\
\hline
\end{tabular}

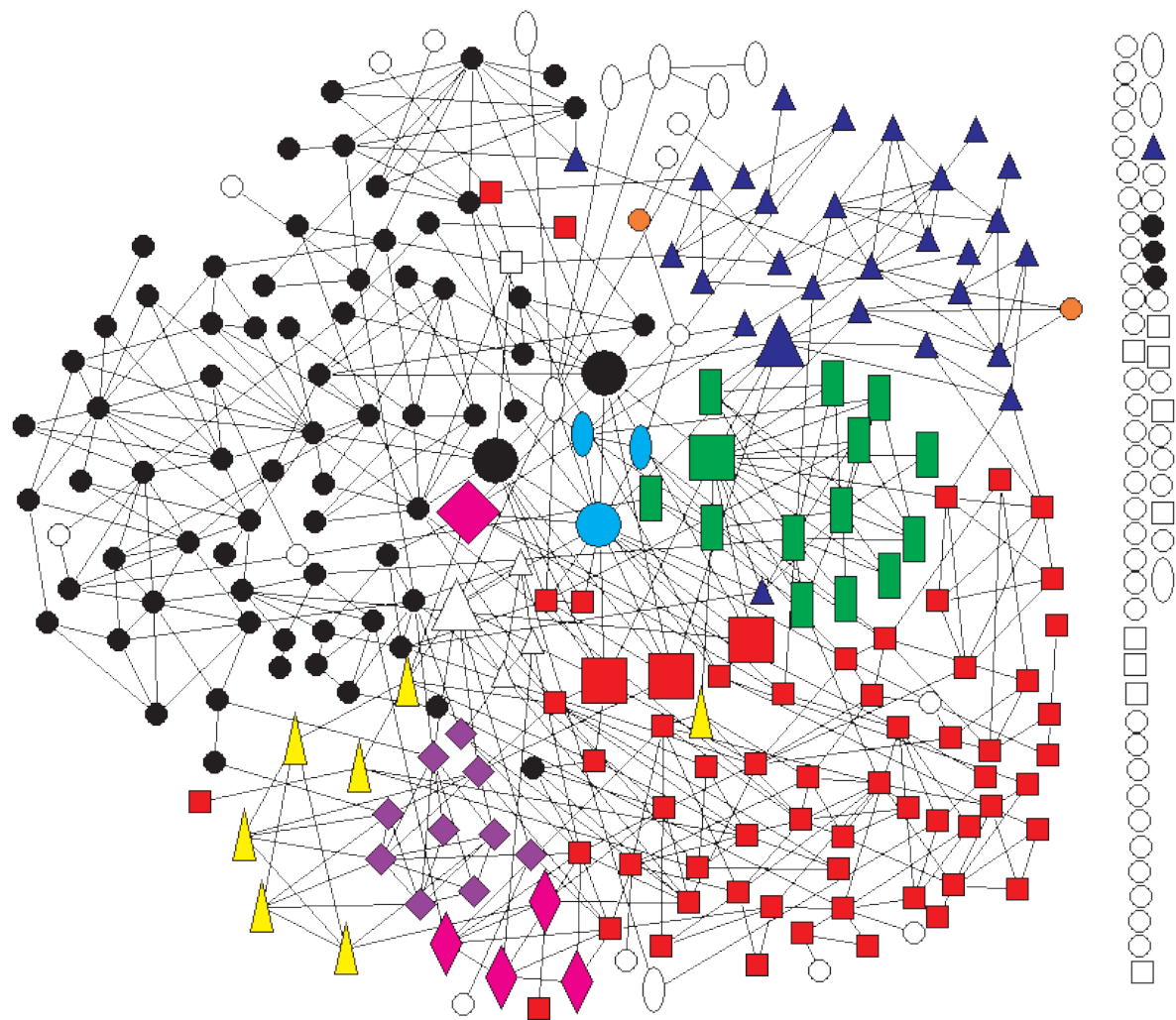

Figure 8.2 Punukula's social network. The big nodes (4 squares, 3 circles, 2 triangles and a diamond) represent people with the ten highest closeness centrality in Table 8.2. The shape scheme used for representing the castes is the same as in Figure 8.1. Unfilled nodes represent people residing outside the village. Isolates are shown on the right side of the Figure. 
Other socioeconomic characteristics of the set of individuals listed in Table 8.2 are discussed at the end of section 8.3, after I have identified members of the core using the social capital measure in combination with the present centrality measure.

Finally, commonly used measures of centrality are unable to capture the importance of inter-caste links that act as bridges between the different social groups to make collective action possible in Punukula. ${ }^{19}$ In order to capture this importance of inter-caste links, I use a second measure, of social capital, largely based on Burt's $(1992 ; 2000)$ theory of structural holes. Therefore, I combine the measure of closeness centrality discussed in this section with a measure of an individual's potential to broker across structural holes or divides.

\subsubsection{Brokerage and social capital}

At the outset here, I must consider the work of Gould (1990; Gould and Fernandez 1989) on brokerage and power in networks. The idea of "brokerage behaviour" as a source of power was demonstrated in a mathematical model by Marsden (1982): ${ }^{20}$ this forms the basis of Gould's (1990) discussion. Brokers are deemed to be most influential and powerful in places where they mediate between groups that are separated by socio-cultural factors such as caste (Gould 1990). The separation between the groups can be horizontal cleavages or vertical hierarchical divisions. Finally, the power of brokers "corresponds to the kind of "agenda-setting" power discussed by Bachrach and Baratz (1962) and by Crenson (1971)." (Gould 1990: 535). To measure this type of 'structural' power, Gould (1990) suggested an index of inter-clique betweenness centrality.

Gould's (1990) discussion on power through brokerage appears to be a good description of the activities of some core individuals in Punukula, and the importance of their inter-caste ties in this process. I do not however use Gould's (1990) index of inter-clique betweenness or "brokerage potential" due to the ambiguity involved in identifying cliques in Punukula: ${ }^{21}$ as discussed above, caste or lineage-based identification of cliques is not straightforward in this village. In addition, sub-dividing the social network using structural techniques, such as hierarchical decomposition, is a cumbersome technical task and not always accurate. The problem however is

\footnotetext{
${ }^{19}$ Betweenness centrality is generally believed to be a suitable measure for this purpose (see Freeman 1979). But as I discuss later, it only measures the extent to which an individual node controls the communication between other individuals rather than groups.

20 Marsden (1982: 202) defines brokerage as a process "by which intermediate actors facilitate transactions between other actors lacking access to or trust in one another." The term transaction here refers to "any form of instrumental relation," including "mediation functions", rather than a simple exchange of resources (Gould 1990: 533). Brokerage behaviour then does not need to be necessarily compensated, i.e. reward for the "intermediaries is untied to any specific transaction." (Gould 1990: 533). The last implies that brokerage can occur through social relationships, where the reward for the broker is ambiguous and must be expected, if at all, over long time frames.

${ }^{21}$ Gould (1990) had extensive data, including information about cliques and sub-cliques, for two small cities studied earlier by Laumann and Pappi (1976).
} 
circumvented by using a straightforward measure due to Burt (1992; 2000) which allows one to capture the influence derived through brokerage between cliquish groups (or across 'structural holes') in social networks. Burt (2000) however does not call this structural status derived from potential for brokerage as power, but social capital.22

The French sociologist Bourdieu (1986) was one of the first to provide an exposition of the term social capital. For Bourdieu (1986), the social world can only be understood by accounting for capital, and its accumulation, in all its forms. ${ }^{23}$ Three different forms of capital are important in this respect: first, the traditionally employed notion of capital as economic capital which is money or commodities in its circular process of production and other financial assets; the second cultural capital is more diffuse, existing in the form of cultural goods such as books and paintings in addition to educational qualifications, knowledge and symbolic cultural capital in the form of power to set artistic standards and tastes (Anheier et. al. 1995); and finally social capital refers to the benefits an individual derives out of the membership of networks of "mutual acquaintance and recognition" (Bourdieu 1986:248). All of the above different forms of capital are convertible into each other under certain conditions (in addition to Bourdieu's 1986 original work, see Anheir et al. 1995 for a clear summary of the different forms of capital and their convertibility).

Since the pioneering work of Bourdieu (1986), social capital has been extensively studied in sociology, business studies, economics, and political science (see for example, Portes 1998; 2000; Portes and Landolt 2000; Coleman 1988; Lin 2000; Lin and Dumin 1986; Burt 2000; Woolcock 1998; Putnam 1993; and the collection of papers in a World Bank study edited by Dasgupta and Serageldin 1999). Social capital as originally treated by Bourdieu (1986) is something held, and benefits from it are accrued, by individuals. In an influential study, Coleman (1988) brought the focus away from individuals to social capital of groups. In a later avatar, social capital was put forward as a cause, and effect, of economic development and 'good' governance of cities and nations (see Putnam 1993). Accompanied with this usage of social capital, as

22 Burt's (1992; 2000) use of the term of social capital ignores one of its two essential components, namely the content of links. According to Burt, the network structure component of social capital by itself has adequate explanatory power. For example, in Burt (1992), he maps the different content of ties but does not use tie content as a factor in his (structural) analysis of managerial performance. As I discuss in the following, Burt's interpretation of social capital is one of many ambiguous and often contradictory uses of the term in the existing literature.

23 Bourdieu (1986: 241) defines capital as "accumulated labor (in its materialized form or its 'incorporated' embodied form) which, when appropriated on a private, i.e. exclusive, basis by agents or groups of agents enables them to appropriate social energy in the form of reified or living labor." Bourdieu (1986) tends to equate capital with power. According to Smart (1993: 390), elsewhere Bourdieu (1984: 114) defines capital as "the set of actually usable resources and power." "The latter definition seems to be the way the term is used in practice: capital is anything that can be used to influence the behaviour of others or to aid in achieving desired goals." (Smart 1993: 390). Therefore, in this latter sense, the concept of capital, or social capital, is similar to the concept of power discussed earlier in this section. 
an asset owned by actors at multiple levels, is an unqualified usage as a source of a variety of effects: according to Portes (2000), social capital has been used to explain everything ranging from the efficiency of government in cities and nations to the performance of individual children at school. This has led to a great degree of ambiguity in the definition of the term, which in turn has limited its analytical power (Fine 2001). A few studies from the mid-1990's onward have tried to clear some of the confusion surrounding the term. Among these, notable is the work of Portes (1998; 2000; Portes and Landolt 2000), who has dug into classical sociological theory to distinguish between the sources and effects of social capital. Other important studies have been performed by Woolcock (1998); Lin (2000; 2001); and Szreter (2002). Following Bourdieu's original work, I view social capital as an asset of individual households rather than groups or nations. This focus at the individual level is suitable for the present purpose of identifying individuals with high social capital in Punukula.

Social capital of an individual can be decomposed into two elements, both of which appear in Bourdieu's original concept (Portes 1998). According to Bourdieu (1986: 249),

"The volume of social capital possessed by a given agent thus depends on the size of the network connections he can effectively mobilize and on the volume of the capital (economic, cultural or symbolic) possessed in his own right [by each of those] to whom he is connected." (Italics mine). ${ }^{24}$

The two elements then are the size (or more generally, the structure) of the social ties of an individual through which she can gain access to resources, and the quality and quantity of the resources held by the people she is connected to. A third element that may be included in an extended conceptualisation of social capital pertains to the utilization of the resources accessed by an agent (through her social ties) (see Lin and Dumin 1986 for a similar conceptualisation although they do not use the term social capital specifically). The three elements may or may not be correlated with each other.

In this Chapter, my focus is on the first element of social capital pointed out by Bourdieu (1986): the structure of an individual's network connections. ${ }^{25}$ In the existing literature on sociology of network, the network structure of social capital is defined in two ways: closure and structural holes. Closure in networks follows from Coleman (1988), who focussed on social capital as a source of social control (Portes 2000).

${ }^{24}$ Bourdieu's definition of social capital is of course one of many available in the literature: I use it because of its appropriateness for the present analysis of social network among individuals. Other definitions have, for example, taken social capital to mean norms (Coleman 1988); or membership of voluntary organizations (Putnam 1993). Paldam (2005: 2) identifies three main definitions of social capital. The first is related to the "ability of people to cooperate voluntarily" without imposition from a hierarchical authority; the second is network density; and the third is trust, both "generalized trust in people and special trust in neighbours and in authorities." Paldam's second definition must be extended beyond network density to include other features of network structure such as bridging.

25 I study the second element in Bourdieu's social capital in Chapter 9. In particular, I use the three different network maps of Punukula to study the provision and access to knowledge through noneconomic ('social') ties of kinship and friendship. 
According to this network closure argument, networks in which people are tightly interconnected allow social control to work. In such networks, "no one can escape the notice of others." (Burt 2000: 6). Network closure then allows people to sanction others who do not observe the rules of the game. This eventually allows people to trust each other and norms of acceptable behaviour are established (Coleman 1988 uses the example of diamond merchants in New York and rotating credit associations to illustrate this point). To summarize, Coleman (1988) argues that members of groups with dense interconnections possess higher social capital. This type of social capital is also broadly referred to as bonding social capital (see for example, Putnam 2000). Many individuals in Punukula, due to their membership in the village's tight-knit sub-caste groupings shown in Figure 8.1, possess high bonding social capital.

The alternate argument initiated by Burt (1992) underlines the importance of structural holes. Social capital of an individual, like power in Gould's (1990) discussion, depends on an individual's potential as a broker between different cliques or groups. According to Burt (2000), the idea is built on the work on networks by sociologists: the strength of weak ties argument put forward by Granovetter (1973); Freeman's (1979) work on centrality, in particular betweenness centrality; and other work on exchange networks. Burt's $(1992 ; 2000)$ focus is on the weak ties that bridge two, or more, groups. Each of these groups contains strong internal connections among its members. The weak ties then act as structural holes in the entire network (Burt 1992; 2000). The bridging tie over the structural hole does not however have to be weak: according to Burt (1992: 30),

"Bridge strength is an aside in the structural hole argument. Information benefits are expected to travel over all bridges."

In the present discussion, the nature of the social network data restricts my focus to be strong bridging ties. People at each end of a bridging tie possess bridging social capital (cf. Putnam 2000). ${ }^{26}$

The critical trait of a tie that acts as a bridge between two groups is nonredundancy (Burt 1992; 2000). Redundant ties generally provide the same, or similar, information to an individual as her other ties. Thus, an individual with a large number of redundant ties possesses low social capital. There are two indicators of redundancy in networks (Burt 2000: 9). The first is related to cohesiveness associated with densely connected groups: there is a high probability that one's contacts from within the group have similar information. This is Burt's $(1992 ; 2000)$ main point of departure from Coleman's (1988) emphasis on network closure in densely connected groups as a source of social capital. The second indicator, pointed out by Burt (2000), is based on the fact that information sources of structurally equivalent contacts are the same, and

26 Bridging social capital may be usefully categorised in two ways: the first one is derived from brokerage potential between horizontally divided groups (such as the different sub-caste based groups in Punukula), and the second is derived from brokerage across vertical divides such as that between NGO representatives or the all-in-one dealers and the farmers. I follow Szreter (2002) in referring to the former type as bridging and the latter as linking social capital. 
so they create redundancy. Structural holes, by definition, are bridged by nonredundant ties. According to Burt (2000: 9),

"People on either side of a structural hole circulate in different flows of information between people, and control the projects that bring together people from opposite sides of the hole."

The circulating in "different flows of information" in the above make the ties that bridge structural holes as non-redundant; and provide people at the two ends of the hole with brokerage potential. The idea is that people with connections rich in structural holes "know about, have a hand in, and exercise control over, more rewarding opportunities" (Burt 2000: 11). In this manner, the brokers gain "control" of projects that they are involved in, such as the control of decision-making on NPM in Punukula.

Burt (1992) proposed an index, that he called network constraint, to measure the brokerage potential from structural holes. This index measures the extent of redundancy in a person's ties in her social network. The lower the constraint index, the higher an individual's brokerage potential in her network, and thus higher the social capital held by her. Mathematically, the network constraint measure of a node $i$ in the network is given by (Burt 1992; Batagelj and Mrvar 2007),

$$
C_{i}=\sum_{j}\left(p_{i j}+\sum_{k, k \neq i, k \neq j} p_{i k} p_{k j}\right)^{2}
$$

where $p_{i j}$ is the proportion of the strength of the link between nodes $i$ and $j$ as compared to the sum of the strengths of all links connecting $i$ to other nodes in the network. If $a_{i j}$ is the strength of the link from node $i$ to node $j$, then $p_{i j}$ is given by $\left(a_{i j}+a_{j i}\right) / \sum_{k}\left(a_{i k}+a_{k i}\right) \cdot C_{i}$ basically measures the structural holes that $i$ can access using the connection to $j$. A high value of $C_{i}$ implies that the individual $i$ has access to few structural holes.

Once again I use Pajek (Batagelj and Mrvar 2007) to calculate the constraint values for Punukula's social network data. Ten individuals in Punukula with the lowest constraint values (highest social capital) are listed in Table 8.3 and highlighted in the social network drawing shown in Figure 8.3.

Table 8.3 Set of Punukula inhabitants with high brokerage potential

\begin{tabular}{|l|l|}
\hline Name & Network Constraint \\
\hline Gorinta, EG & 0.116391 \\
\hline Sapna, FC & 0.122514 \\
\hline Koli, ED & 0.130197 \\
\hline Joardar, GX & 0.134249 \\
\hline Veena, IG & 0.143957 \\
\hline Seri, AX & 0.147943 \\
\hline Yarla, FQ & 0.154784 \\
\hline
\end{tabular}




\begin{tabular}{|l|l|}
\hline Moti, CQ & 0.157884 \\
\hline Velu, HO & 0.161840 \\
\hline Koli, EB & 0.174040 \\
\hline
\end{tabular}

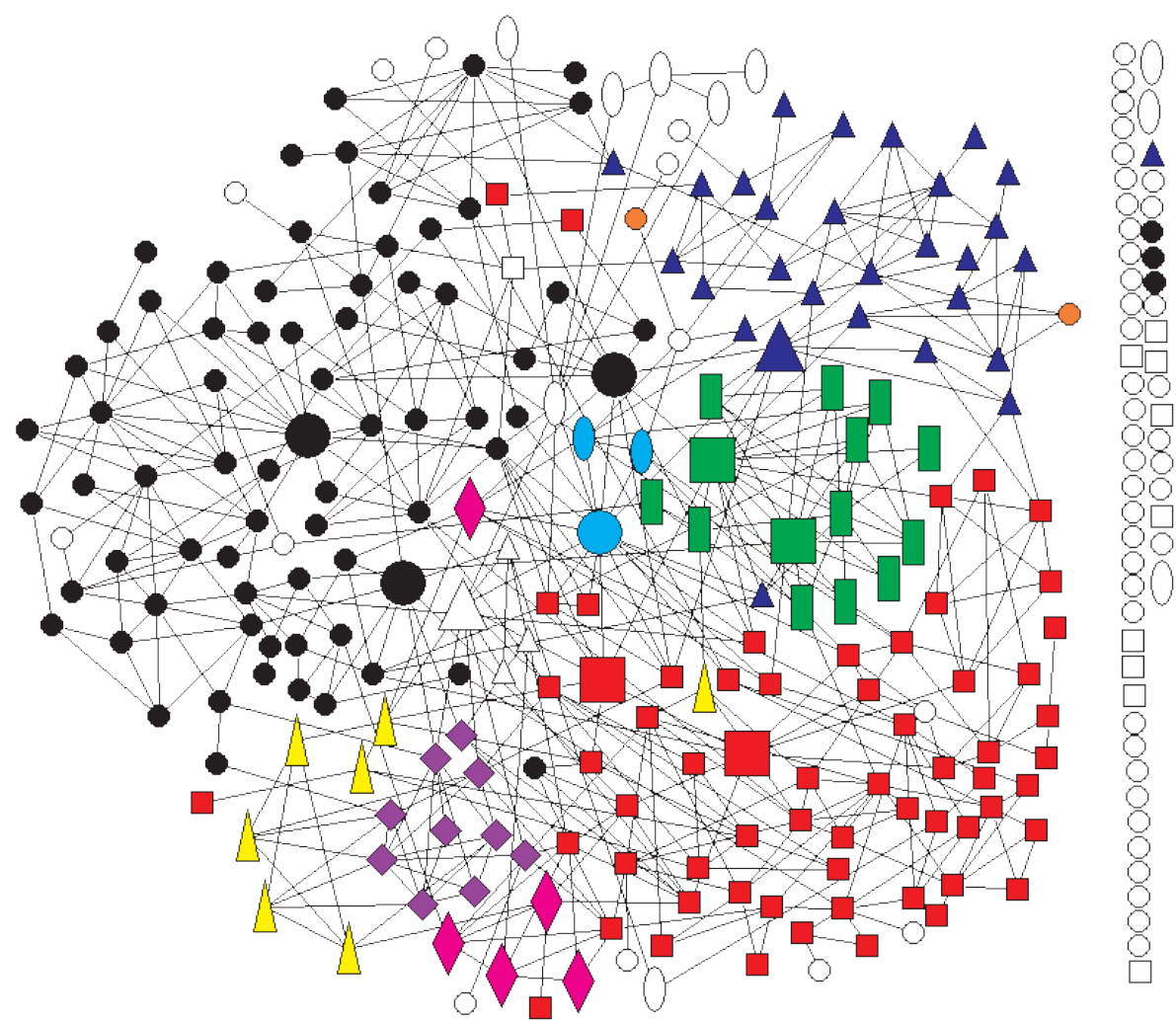

Figure 8.3 People with high network constraint represented as bigger nodes in Punukula's social network. The shape scheme used for representing the castes is the same as in Figure 8.1 (see Table 8.1 for details). Unfilled circles, ellipses (pesticide dealers), and squares (NGO representatives) reside outside the village. Unconnected nodes on the right are isolates in the network.

These inhabitants of Punukula possess high social capital due to their positions in the social network, which afford them potential to broker between (partially) divided groups. There were two divides (or structural holes) across which brokerage opportunities existed in the village. The first divide was horizontal, between the different caste-based groups of Punukula. This brokerage was largely done by people who acted as representatives of their respective sub-caste based groups (almost all people listed in Table 8.3). These people possess high bridging social capital. Secondly, some of the same people acted as brokers between vertically divided groups, for example, between the NGO representatives and the farmers in the village, or between the all-in-one dealers and the village (see Reddy and Haragopal 1985 for the 
importance of this type of vertical brokerage in Indian rural development; and Evans 1996 for a more general synthesis of this vertically structured social capital). These people (Yarla, FQ, Gorinta EG, Joardar GX, Veena IG, Koli, ED) possessed high linking social capital (Szreter 2002). ${ }^{27}$

\subsection{Community leaders in the social network and group action}

To find the potential members of the core in Punukula, I combine the sets of individuals with high closeness centrality and high bridging social capital values, listed in Tables 8.2 and 8.3 respectively. The union of these two sets produces a set of prominent people in Punukula. Six villagers are common to both sets, so the union gives a total of 14 individuals. ${ }^{28}$

Each of the 14 individuals must either possess a high degree of independence, or have a high brokerage-derived social capital, or both of the above. As discussed in the previous section, the two types of prominence, dominance or independence (closeness centrality) and brokerage potential (network constraint) are two approaches to identify powerful brokers in a community. ${ }^{29}$ According to results from part I of the thesis, the 14 most prominent individuals in Punukula can only act as the core of its social network if a circular process (cyclic network structure) exists among them. The structure of connections among these prominent people is shown in Figure 8.4. The names of the individuals along with the numbers used to represent then in Figure 8.4 are listed in Table 8.4.

\footnotetext{
27 As I show later, these categories of bridging and linking social capital are useful in presenting the story of the collective action driven media success in, and the later factional divide that became apparent during the minister's visit to, Punukula.

${ }^{28}$ Note that all of these Punukula residents were identified using structural measures: none of the other measures of socioeconomic power were employed. I briefly compare the structural characteristics with the socioeconomic attributes at the end of this section.

${ }^{29}$ It may be also fruitful to view these individuals as political entrepreneurs. Attwood (1974) identifies two types of political entrepreneurs - the patron and, his competitor, the group mobilizer. The patron is the powerful local person who acts as a middleman across vertical divides (for example, between the farmers and the government agriculture department), whereas the group mobilizer acts as middlemen between horizontally divided groups. Both types of entrepreneurs, through their political activities, make the people dependent on them and play a crucial role in bringing about social change. In addition, according to Attwood (1974), the distinction between the patron and the group mobilizer is not as dichotomous as the vertical/horizontal middlemen story implies. To be successful, patrons often must act as group mobilizers and vice versa. This dual role seems to describe Punukula's case if the power measure of closeness centrality is taken as a rough proxy for being a patron and brokerage potential for a mobilizer.
} 


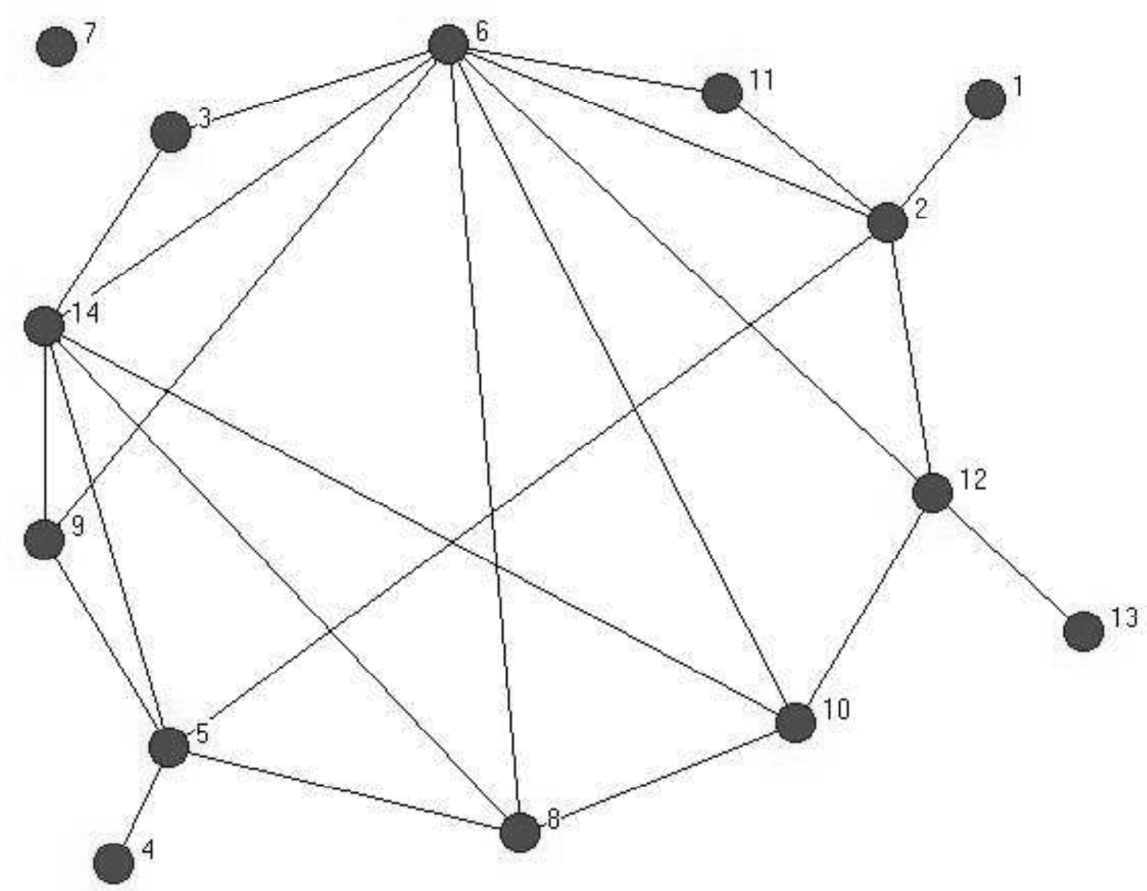

Figure 8.4 The structure of connections in the group of prominent people identified using closeness centrality and network constraint measures.

Table 8.4 List of prominent people in Punukula

\begin{tabular}{|l|l|}
\hline Name & Node number in Figure 8.4 \\
\hline Seri, AX & 1 \\
\hline Moti, CQ & 2 \\
\hline Pai, DJ & 3 \\
\hline Koli, EB & 4 \\
\hline Koli, ED & 5 \\
\hline Gorinta, EG & 6 \\
\hline Sapna, FC & 7 \\
\hline Yarla, FQ & 8 \\
\hline Pillai, GA & 9 \\
\hline Pillai, GB & 10 \\
\hline Joardar, GX & 11 \\
\hline Velu, HE & 12 \\
\hline Velu, HO & 13 \\
\hline Veena, IG & 14 \\
\hline
\end{tabular}


In Figure 8.4, four nodes (numbers 1, 4, 7, and 13) have less than two links and thus do not form part of the circular process. The other 10 together form the dominant core in Punukula's social network, which is shown in Figure 8.5. The core has 10 internal links, in addition to the 10 links required to form the cycle. The density of internal links in this core $\left(\mathrm{d}_{\mathrm{iL}}=0.285\right)$ is approximately the same as the core in the simulation experiments with the ACS model in Chapter 3 (shown in Figure 3.6). Both cores, shown in Figures 8.5 and 3.6, are of higher strength than a simple cyclic core, but weaker than a complete core. ${ }^{30}$

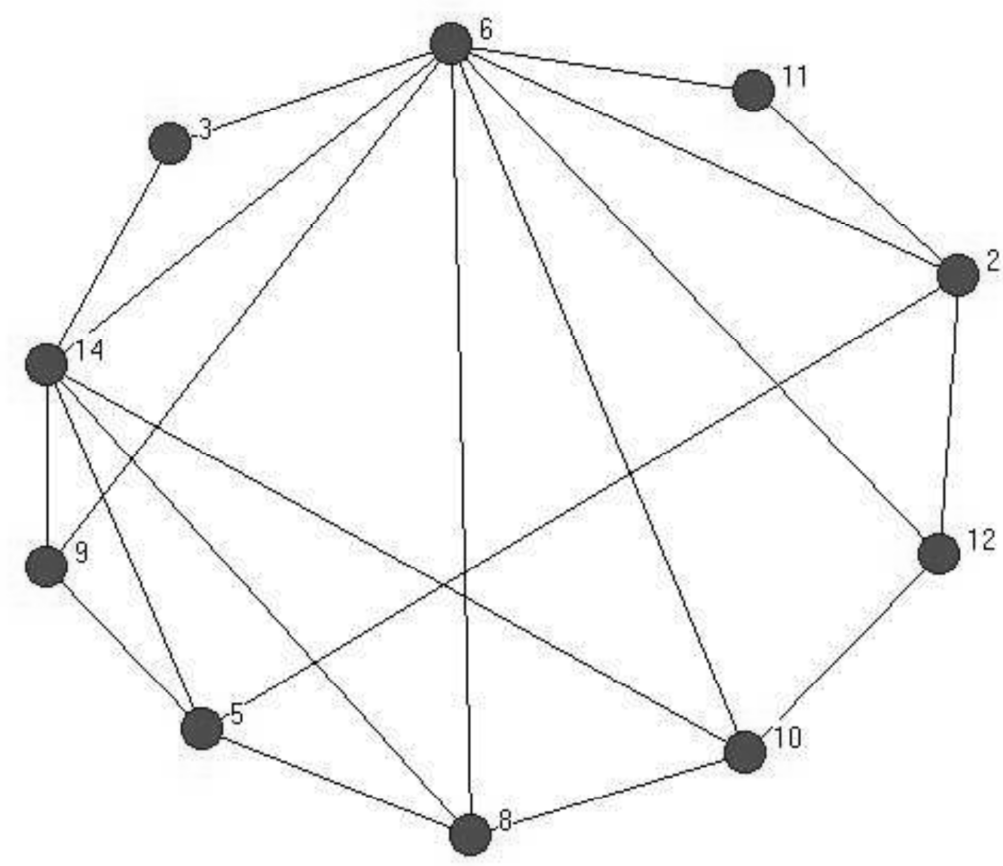

Figure 8.5 The final dominant core formed of ten Punukula residents.

The following individuals form the dominant core in Punukula: CQ Moti, DJ Pai, ED Koli, EG Gorinta, FQ Yarla, GA Pillai, GB Pillai, GX Joardar, HE Velu, and IG Veena. ${ }^{31}$

${ }^{30} \mathrm{~A}$ strong core with many internal links can be split into two or more smaller cores. These smaller cores act as two competing points of power/dominance in the network, as discussed in section 8.5. 31 Note that this eventual group of ten people are the ten with highest closeness centrality values. Only four of these ten people were among the ten with the lowest network constraint. The impact of network constraint in selecting the final core is more pronounced if bigger sets based on the highest values of centrality and lowest network constraint, instead of the present ten listed in Tables 8.1 and 8.2 respectively, are selected. An example of a core formed after the union of initial sets of size 15 is presented in Appendix C. 
The social network of Punukula with the dominant core highlighted is shown in Figure 8.2 (as the final 10 members of the core are those with the highest closeness centrality values). The core residents collectively represent 7 of the 10 caste groups living in Punukula. Five of them are heads of their respective sub-caste communities in the village. According to the NGO workers and some Punukula residents, these five together with the president of the panchayat control the affairs of the panchayat. Most Punukula residents quoted in the media reports are members of the core. Eight of the ten members cultivated a total 71.5 acres in 2005-6: an average of 8.94 acres as compared to an average of 4.85 acres per household for the whole of Punukula. In addition, six are engaged in non-agricultural income generating activities such as dairy farming, local medical practice, and land surveying. Five core members were appointed as resource persons in the village by SECURE and work in close cooperation with the latter in the NPM project (although only one core member has a strong social tie to a NGO representative). Three are close friends of the main pesticide supplier to the village. Together the group of 10 people, identified only on the basis of their structural position in Punukula's social network, certainly form the power centre of the village.

Punukula's core group of elites were the real decision-makers in the village, who may have been induced to work together by the NGO (SECURE). However, it is erroneous to assume that this core group was created de novo by the NGO. For implementing the NPM project in Punukula, SECURE may have selected some individuals as strategic access points to the village. But these strategic individuals were socially related to each other a priori. This existing social organization of a core group of people acted as a fertile ground for SECURE's NPM project in Punukula. The members of this core group brought their collective power and social capital behind the NPM effort, which culminated in the panchayat resolution proclaiming Punukula as free of pesticides and the media publicity.

More fruitfully, however, Punukula's socio-political activities surrounding NPM may be viewed as an alignment of the people with local power at the village level to people with power at the sub-district level, in the realm of development. In social capital terms, members of the core acted as go-betweens between the villagers and the NGO, and as a result, held high linking social capital. ${ }^{32}$ In addition, the core members possessed critical bonding and bridging social capital by being tightly connected within their respective sub-caste based communities (recall that many of them are community headmen) and having links that connect them to important members of other castes (the links within the core for example) respectively. ${ }^{33}$ Bonding social capital allowed them to gain approval, through coercion or consensus, in their respective communities for NPM-related activities. And bridging social capital allowed them to extend this

\footnotetext{
32 Although only one member of the core possessed a direct social tie to a NGO representatives. As mentioned above, five of ten core members acted as resource persons of the NGO signifying their close connection to the NGO, particularly in the NPM arena.

${ }^{33}$ In this way, note that bonding and bridging social capital are prerequisites for effective use and distribution of economic capital converted from linking social capital (cf. Ettlinger 1994).
} 
approval across caste-divides in the wider social network of the village. The latter two types of social capital possessed by the core members created Punukula's face of unity and success observed in the media. Access to media and NPM was of course achieved and made successful through linking social capital of the core members.

In this section, I have attempted to identify features of Punukula's social structure that made collective action in the village possible. I argued that collective action was led by a group of prominent people who were socially connected to each other as a structural core in Punukula's social network. This core group of people provided the necessary combination of three types of social capital that produced both the panchayat resolution and the media success. Unlike Wade (1988), who emphasized issues of natural risk and scarcity as drivers of collective action in AP villages, I have underlined the importance of social structural factors.

\subsection{The rift in the face of unity}

In this section, I attempt to construct a social network based explanation for the rift in Punukula, which became evident during the AP agriculture minister's visit. Two alternate stories regarding the success of Punukula farmers in avoiding pesticides surfaced during the minister's visit. According to the first version, farmers in Punukula stopped using pesticides largely on their own initiative, with little help from SECURE. The second version highlighted the role played by SECURE and the availability of a viable alternative in NPM as critical to the transition away from pesticides. The two versions were put forward by two different groups of prominent people in Punukula: the first group was structured around the panchayat president and some other people close to the main pesticide supplier to Punukula, whereas the second group was largely composed of Punukula residents acting as SECURE's resource persons.

In the context of the core-periphery structure of Punukula's social network, I argue that the divide arose because of a split in the core. High strength of the core, due to multiple internal links, allows the core to be split into smaller cyclic structures which then act as separate competing cores. For the core shown in Figure 8.5, two smaller cores of sizes after such a split are formed by nodes $6,11,2$ and 12 on the one hand, and nodes 10, 8, 5, 9, and 14 on the other. These separate competing cores led two factions in Punukula. Three of the four people in the first split core are directly connected to the main pesticide (or all-in-one) dealer for Punukula. One of these three people is the president of the gram panchayat. The second small core after the split is composed of village elders from different communities, who acted as SECURE resource persons. They spoke on SECURE's behalf and told the NPM version of the no-pesticides story to the agriculture minister.

As discussed in $\$ 8.4$, links within the dominant core of Punukula's social network provide access to two types of social capital in Putnam's (2000) typology: bonding and bridging social capital. Furthermore, links of the core-members to the outside (to NGO representatives and the all-in-one dealers for example) provide linking social capital (Szreter 2002). As discussed in chapters 6 and 7, most farmers use these outside 
ties to get access to credit, knowledge, and other critical resources for themselves and their kin/friends. ${ }^{34}$ In the first split core, three of four members possess direct (strong) social ties with the main all-in-one dealer in Punukula, M. Raj. Thus, access of economic resources (credit, farm-inputs, knowledge) of these actors were embedded in their social ties characterised by mutual respect, trust, and long-run obligations. ${ }^{35}$

In contrast, members of the second split core do not possess any direct (strong) social ties to NGO representatives. ${ }^{36}$ Therefore, the arrangements between the NGO and its resource persons were not embedded in direct social ties, and did not create strong linking social capital to compete with that of the first split core. I argue that this lack of embeddedness of the 'vertical' arrangements, between the farmers and the NGO, in strong social ties in the second split core led to the fall in NPM use after its initial success in Punukula (see Chapter 6). This lack of embeddedness of the formal farmer-NGO arrangements in strong social ties also allowed a resurgence in control of the all-in-one dealer through the competing split first core. Therefore, social ties matter for technological innovation, in particular to stabilise the innovation and make it a sustainable alternative to any competing technologies, and they do so by embedding formal resource exchange arrangements in durable interpersonal relationships.

To summarize, the core members are aligned to powerful individuals and organizations from outside the village, which can create a conflict in the dominant core of the village's social network. The rift in the village may reflect a wider conflict of interests between these outside individuals and organizations. Some of this vertical alignment is 'visible' through social connections of some core members to the main pesticide supplier to the village. However, other forms of alignment, such as the local NGO's arrangements with some core members, are not visible in Punukula's social network with its focus on strong social ties. The interplay between these (formal) arrangements and the (informal) social ties plays an important role in regulating group action by the villagers, as witnessed in the eventual factionalizing of the village (and the split of the original dominant core in the social network). Finally, I argued that the embeddedness of NGO-farmer arrangements in strong social ties is necessary to create adequate linking social capital for competing with the strong 'alignment' of the all-inone dealer with some members of the core.

\subsection{Conclusions}

In this chapter, first I argued that an exclusive focus on inter-caste relations and caste-based hierarchy, by neglecting the possibility of new important social groupings

34 This conceptualisation that includes the economic resources converted from some features of social relations leads to a complete definition of Bourdieu's social capital (by bringing the content of links in the social network into the picture).

${ }^{35}$ Embeddedness of resource exchange links, in particular knowledge flows, in strong social ties is the focus of Chapter 9.

36 The only farmer who possesses a strong social tie with a NGO representatives is also a member of the first split core. 
and interpersonal relationships, provides a caricatured explanation of group action in rural India. According to this view, in a traditional village, one would expect the elders of a dominant caste to govern and act as the village spokesmen. In Punukula by contrast, I find that elders from a few castes share power in the village to act as its spokesmen and leaders. Similar power sharing among castes as the organizing principle, instead of one dominant caste, has been observed in earlier studies (see for example Dube 1968 and Cohn 1987 cited in Mendelsohn in 1993: 806).

Working then with a multi-caste interpersonal relationships perspective, I traced the roots of collective action in Punukula to the activities of a group of core people in the village. These powerful leaders, some of whom are headmen of their caste-based groups, held wide influence over the villagers as shown by their high closeness centrality in Punukula's social network. In addition, they effectively brokered between different caste-based groups, and between the village and important outside agencies. Structurally, this brokerage potential of the core people was reflected in high values of their network constraint values.

The core group of prominent people in Punukula used their inter-caste links to sew the village together, and brought the NGO closer to the village, thus realizing the observed collective decision-making around the no pesticides resolution and the unified representation of NPM success to the media. They possessed the requisite bridging and linking social capital producing the 'social' success in Punukula. ${ }^{37}$

I argued that the eventual factionalizing of the village into two camps can be explained by a split in the core of Punukula's social network into two smaller cores. The two smaller cores were aligned to opposing interests from outside the village - the main pesticide supplier and the NGO responsible for promoting the alternative technology of NPM. Eventually I argued that the smaller core aligned with the pesticide supplier turned out to be stronger of the two due to the presence of the strong social ties between the pesticide supplier and the farmers. This embedded the 'formal' (in this case, economic) exchange ties of the supplier with the farmers in strong social relations. A corresponding embeddedness of the NGO-farmer exchange arrangements in social ties was not observed. ${ }^{38}$

\footnotetext{
${ }^{37}$ Note that the focus of this chapter was only on the structural component of social capital. The content of links in a network, or the nature of resources transferred is considered in chapter 9 .

38 Embeddedness at the individual and network levels is studied in more detail in the next chapter of the dissertation, where the factors observed here emerge more clearly.
} 


\section{Embeddedness of knowledge flows in social networks}

The aim of the present chapter is to study the embeddedness of knowledge flows in the village. The degree of embeddedness is given by the proportion of total knowledge flows which occur through social relations. Social relations are conceptualised narrowly here, as kinship and friendship ties of the villagers. ${ }^{1}$ Such ties are characterized by mutual respect, conviviality, trust, and long-term reciprocity.

Embeddedness in social ties undergirds social capital. In order to avoid confusion due to the plethora of interpretations in the literature, ${ }^{2}$ I conceptualise social capital by focussing on social (kinship, friendship) ties that provide access to knowledge directly or indirectly. The availability of concrete data on the knowledge-flows and social networks among the same set of people facilitates this specific application of the two wide-ranging concepts of social capital and embeddedness.

Why is it important to study the embeddedness of knowledge flows in social ties that transmit trust or conviviality? It is widely believed that social networks act as the foundation on which effective knowledge sharing, and the resulting interactive learning, takes place. The location of these embedded learning processes may be inside firms, industrial clusters, innovation systems, or communities of practice. ${ }^{3}$ Consistent with most literature on innovation systems, the undivided knowledge, business and social networks of the village can be treated as a local innovation system which includes the farmers, NGO representatives, private firms, government representatives, and banks. ${ }^{4}$ If actors from outside the village are removed from these undivided networks, we reduce the networks to represent a 'community of agricultural practitioners' inside the village. In this chapter, I present results on the embeddedness of knowledge flows in the local innovation system and the agricultural community.

The chapter is structured as follows. I start with a critical overview of the literature on embeddedness of economic and technological activities in the following section,

1 The importance of social relations for learning and innovation is well accepted (see footnote 3 below for references from several fields). For studies that have highlighted the importance of social relations in the narrow sense, such as the friendship ties considered here, see for example Saxenian (1994); Lave and Wenger (1991); Brown and Duguid (1991); and Amin and Cohendet (2004).

${ }^{2}$ For a critical review of the multiple, inconsistent and often contradictory, interpretations of social capital, see Fine (2001).

3 On embeddedness of interactive learning in firms, see for example, Kogut and Zander (1992, 1996), Uzzi and Lancaster (2003); on industrial clusters, see Saxenian (1994), Schmitz and Nadvi (1999), Asheim and Coenen (2005); on social embeddedness in innovation systems, see for example, Cooke (1998); Coriat and Weinstein (2002); and Lundvall et al. (2002); on communities of practice, see Lave and Wenger (1991); Brown and Duguid (1991); and Amin and Cohendet (2004).

${ }^{4}$ For a critical assessment of viewing innovation systems as networks, see Kaufmann and Tödtling (2001). 
briefly discussing how social capital is derived from embedded ties. ${ }^{5}$ In section 9.2, I present some simple measures of embeddedness and social capital at the individual and network levels. In section 9.3, I study the embeddedness of knowledge flows in the local innovation system which refers to the set of undivided networks of the village. Section 9.4 explores the embeddedness of knowledge sharing in the community of agricultural practitioners in the village. Finally, a fifth section provides some concluding remarks and implications of the present results beyond the village studied.

\section{I Embeddedness of economic and technological activities, and social capital ${ }^{6}$}

The first use of the term embeddedness in the social sciences is generally attributed to Karl Polanyi (1944; 1957). In his classic work, The Great Transformation, Polanyi (1944 [2001]) argues that economic transactions are embedded in sociopolitical and cultural exchanges. More generally, an economy is "embedded and enmeshed in institutions, economic and non-economic.” (Polanyi 1957 [1995:34]). An "economy is not autonomous, as it must be in economic theory, but subordinated to politics, religion, and social relations.” (Block 2001: xxiv). Disembedding the market from social relations has never succeeded in history for an extended period of time.

${ }^{5} \mathrm{My}$ focus is largely restricted to the literature on interpersonal and inter-organizational networks in the sub-discipline of economic sociology. The reason for this focus is simply related to the extensive treatment of the concepts 'embeddedness' and 'social capital' in economic sociology (where studies on social networks have literally boomed since the 1980s). Probably as a result of this focus, I overlook other literatures on networks, for example in economics, which do not directly impinge on my arguments about economic sociology. Thus, game theoretic and experimental approaches to networks are not discussed in the following. However, I must refer to an important study that is in some respects similar to mine: analysing multiple networks (with different tie contents) in a rural context (D'Exelle and Riedl 2008). They use maps of networks, in a Nicaraguan village, with six different types of ties (ranging from friendship and support to economic and public activities) to explain generosity (assessed using an experiment with all household heads in the village). Consistent with other literature on giving and friendship networks, they find that geodesic distance is important for generosity. However, distance in networks other than the friendship network has no influence on giving behaviour. Alternate network structure variables such as closure however do matter in the other networks.

${ }^{6}$ Most of this section focuses on the all-inclusive concept of embeddedness by reviewing its various types and dimensions. Using this review, I arrive at a specific type of embeddedness, and its dimensions, which is suitable for the present analysis. A similar introductory review on social capital is available in the previous chapter of this thesis where I discuss bonding, bridging, and linking social capital. The network structure component of social capital was the focus there, whereas the tie content was restricted to traditional non-economic resources associated with social capital such as trust, solidarity, and mutual respect. In this chapter, I study whether the same non-economic or 'social' ties also act as conduits of the important economic (and technological) resource of knowledge, thereby measuring what I call social capital for knowledge. 
Complete disembedding cannot take place without the destruction of society and the natural environment (Polanyi 1944 [2001]; Block 2001). ${ }^{7}$

There has been recent upsurge of interest in embeddedness, largely due to an important article by Granovetter (1985). According to Swedberg (1997), Granovetter's article signalled the birth of the new sub-discipline of economic sociology. Granovetter builds his arguments about embeddedness on a critique of the under socialised conception of behaviour in mainstream economic theory and the over socialised conception of some sociologists. Both views neglect the impact of ongoing social relations on economic transactions by focussing on atomized actors,

"In the under socialized account, atomization results from narrow utilitarian pursuit of selfinterest; in the over socialized one, from the fact that behavioural patterns have been internalized and ongoing social relations thus have only peripheral effects on behaviour." (Granovetter 1985: 485).

Granovetter (1985) then argues that analyses of economic action must reject the idea of atomized actors: actors undertake economic transactions and other activities while embedded in "concrete, ongoing systems of social relations." (Granovetter 1985: 487).

Granovetter (1985) briefly outlines two forms of embeddedness: historical (now often called relational) and structural. Historical embeddedness focuses on a dyad and is related to repeated transactions between two actors. The history of transactions along the dyad provides low-cost reliable information to the two connected actors about each other. Eventually the tie gets buttressed with non-economic factors such as trust and reciprocal obligations (Granovetter 1985: 490). Structural embeddedness, on the other hand, focuses on the position of an individual dyad in a broader network of relations. Transactions in the dyad are then constrained and/or supported by the social structure (network) in which it is embedded.

Following Granovetter (1985), a large number of studies in economic sociology have attempted to further develop the concept of embeddedness. For example in the last two decades, the concept of embeddedness has been used to explain the effect of social relations on the performance of organizations (Uzzi 1996; 1997; Rowley et al. 2000); the logic of negotiations (McGinn and Keros 2002); economic activities of immigrants (Portes and Sensenbrenner 1993); consumer transactions (DiMaggio and Louch 1998); community cohesion and solidarity (Moody and White 2003); and the role of the state in the economic development of nations (Evans 1995; 1996). Related work has focussed on theoretical development of the concept of embeddedness (see for example, Zukin and DiMaggio 1990; DiMaggio and Louch 1998; Gulati 1998). I review these theoretical refinements below. Despite this large body of work, the theoretical underpinnings, and conceptual clarity, of embeddedness in economic sociology has remained weak (Portes and Sensenbrenner 1993; Fine 2001; Krippner

7 However, different interpretations of Polanyi's arguments exist in the literature. For example, Lie (1992) argues that Polanyi treated market as disembedded from social institutions. See Krippner (2001) for a detailed treatment of different misreadings and interpretations of Polanyi's work. 
2001). According to Portes and Sensenbrenner (1993: 1321), the concept of embeddedness brings about a persuasive critique of neoclassical economic theory but "suffers from theoretical vagueness" when used to provide "concrete propositions." Further theoretical development of the concept is only possible if we are able to specify how "social structure constrains, supports, or derails individual goal-seeking behaviour." (Portes and Sensenbrenner 1993: 1321).

According to Portes and Sensenbrenner (1993), ideas from classical and new sociological theory can be used to theoretically develop the concept of embeddedness. ${ }^{8}$ Casting their arguments in a similar vein, Zukin and DiMaggio (1990) use a broad theoretical foundation to discuss embeddedness: Here, in addition to being embedded in social relations, economic action is viewed as being contingent on human cognition, culture and political institutions. Cognitive embeddedness refers to the "ways in which structured regularities of mental processes limit the exercise of economic reasoning." (Zukin and DiMaggio 1990: 15-16). This type of embeddedness is related to the concept of bounded rationality which forms one of the cornerstones of evolutionary economics (Nelson and Winter 1982). The second, cultural embeddedness implies that economic behaviour (rationality) and market exchange is shaped by cultural factors such as norms, beliefs, rituals, ideologies, and shared understandings of the real world (Zukin and DiMaggio 1990; also see Polanyi 1977; Block 2001). The third, political embeddedness, refers to "the sources and means of economic action that reflect inequalities of power." (Zukin and DiMaggio 1990: 20). Here, economic institutions and behaviour are considered to be shaped by a power struggle involving economic and non-economic actors and institutions such as the state and social classes (Zukin and DiMaggio 1990). This type of embeddedness has been extensively studied by political economists and the old institutionalists (see for example, Commons 1924; and Davis 1949; for recent syntheses, see Scott 2001 and Parto 2005). The final type of embeddedness outlined by Zukin and DiMaggio (1990) ties in with Granovetter's (1985) focus on structural embeddedness of economic action in networks of social relations discussed earlier. Note that here tie content and network structure are considered to be two components of structural embeddedness. My focus in the present chapter is on this last type of embeddedness.

Structural Embeddedness

The literature on structural embeddedness distinguishes between non-anonymous, non-market (embedded) exchanges that take place between social contacts or acquaintances and market (arms-length) transactions that are coolly rational and impersonal (see for example, Uzzi 1996; 1997; Uzzi and Lancaster 2003; Inkpen and Tsang 2005; and Krippner 2001 for a critical assessment of this view). Since few

\footnotetext{
8 Portes and Sensenbrenner (1993) go on to focus on social capital derived from embeddedness, and outline four possible sources of social capital by borrowing ideas from classical sociological theory of Marx, Durkheim, Simmel, and Weber. As discussed in the previous chapter, the four sources are value introjection, reciprocity transactions, bounded solidarity, and enforceable trust. Some of these sources were criticized as "oversocialized" conceptions by Granovetter.
} 
transactions happen in isolation from others, the focus is a network of transactions. In theory, this network of economic transactions is embedded in a corresponding network of social relations. And in the case of embedded transactions, as opposed to arms-length ones, all traceable economic transactions simply double as social ties characterized by non-economic factors such as trust, respect, and long-term reciprocity. This all-inclusive embeddedness ignores that economic transactions may be characterized by different types and degrees of non-economic 'glues' that lend them an air of the benevolent 'social'. Strength of this social glue available due to the nature of social relations, that embed the economic transactions, may vary from almost nil to very strong. Furthermore, some other economic transactions may instead be governed by non-economic factors such as unequal (bargaining) power, conflict, and lifelong dependence rather than trust or mutual respect. Thus in a general formulation, that recognizes a range of often-contradictory non-economic factors, one can only talk about the degree of embeddedness of economic transactions in certain types of social relations, such as those characterized by trust and respect, or unwanted obligations and dependence, or simple indifference. ${ }^{9}$ Therefore, in subsequent sections of this chapter, I focus on the degree of embeddedness of knowledge flows in one type of social relations (close friendships and kinships characterized by trust, companionship, and mutual respect). ${ }^{10}$

Structural embeddedness has two critical components: network structure and types of social relations or content of the links in the network (Uzzi 1996; 1997; Schweizer 1997; Rowley et al. 2000). ${ }^{11}$ Economic sociologists have written extensively on network structure in the last two decades. Their emphasis has been on identifying structures that afford performance advantages to strategically positioned actors in the networks: performance advantages may occur as a result of an actor's potential to control, broker, or simply access superior resources (see for example, Coleman 1990; Burt 1992; 2000). Many economic sociologists use the term 'social capital' to present their arguments, which may be viewed as a consequence of the social embeddedness of economic transactions. The focus has largely been restricted to the two structural

${ }^{9}$ Some of these features of social ties may even coincide. For example, social ties characterized by trust and solidarity may also convey unwanted obligations and claims (see Portes and Sensenbrenner 1993).

10 This is related to Schweizer's (1997) discussion of domain embeddedness, which results from the "interpenetration of societal/ cultural domains". Here I study the interpenetration of the technical domain (knowledge for pest control) with the social domain of close kinship and friendship relations. 11 The latter component is often treated as relational embeddedness in recent literature. This has led to two separate sub-streams in the literature. The first on 'structural embeddedness' focuses predominantly on network structure and the second on relational embeddedness studies the impact of tie content (generally reduced to the strong vs. weak tie distinction). But as noted by Rowley et al. (2000) and others, these two types of embeddedness are interdependent and it may be erroneous to treat them independently. In my conceptualization, what is generally treated as distinct relational embeddedness is a part of structural embeddedness. 
sources of social capital in 'network closure' and 'structural holes' (see chapter 8 for an extended discussion and overview of this debate).

As compared to the extensive research on network structure, accompanying work on the second component of structural embeddedness, the 'content' of ties in a network, has generally been fragmentary and vague (Krippner 2001). ${ }^{12}$ This lack of clear specification of tie content limits the usefulness of network studies because the two components of embeddedness are interdependent (Podolny and Baron 1997; Rowley et al. 2000; Rodan and Galunic 2004). ${ }^{13}$ Furthermore, most economic sociologists use the same constructs about network structure (e.g. closure, brokerage or structural holes) as bases for their arguments irrespective of what flows through the ties of the examined networks. However, as shown by Podolny and Baron (1997) for the case of intra-organizational job mobility, this conflation of different tie-contents under a single social network structure can yield erroneous results. In the organization studied by them, the effect of structural holes on upward job mobility in the workplace is positive if ties in the network transfer resources/information, and negative if tie content is social support or expectations. Podolny and Baron (1997) then build a typology of content of ties in an attempt to generalize the result that the network structure-performance relationship can differ with tie content. In a similar vein, Rodan and Galunic (2004) show that network structure (or sparseness, their proxy for structural holes) does not matter for managerial innovativeness if knowledge heterogeneity (proxy for tie content) is taken into account. In other words, innovation performance depends on access to diverse knowledge types, irrespective of the structure of the network (structural holes or closure). ${ }^{14}$ Thus tie content, rather than network structure, drives their explanation for successful managerial innovation.

It is clear in the foregoing that the performance impact of embeddedness of economic transactions in social ties may differ according to the content of the latter (and the former). For example, only rarely do resource-exchange ties simply double as

12 Often network structure drives explanations even when tie content is specified. Or the latter takes charge, as in studies on relational embeddedness, without a parallel analysis of network structure. For examples of exceptions, see Granovetter (1973); Wellman (1983); Wellman and Wortley (1990); Podolny and Baron (1997); Rowley et al. (2000).

13 As discussed in chapter 8, for social capital, the interdependence between tie content and network structure is often reduced to a simple correspondence: strong ties among close friends and kin form networks whose structures create closure (bonding social capital). Whereas weak social ties, such as those between casual acquaintances and friends of friends, connect structural holes and provide access to novel information not generally available through strong ties (bridging social capital). In this way tie content is reduced to network structure, and vice versa. Thus, parallel analysis of network structure and tie content becomes a rare occurrence.

14 Rodan and Galunic caution against the assumption of simple correspondence between network structure and tie content (see the previous footnote). In their study, structural holes (or sparse networks in their case) are not "perfect surrogates for knowledge heterogeneity" (2004: 556). 
strong social bonds of close friendship and kinship relations. ${ }^{15}$ Therefore, referring to economic exchanges as (embedded in) social relations must be accompanied with a precise qualification of both the type of social relation and the nature of economic exchange: thus, an exact description of 'what is embedded in what'. As noted earlier, this qualification is important because a social tie, in which the economic transaction is embedded, can range from strong kinship ties involving mutual trust and reciprocal obligations over decades to transitory casual acquaintances or hierarchical exploitative relationships. Such disparate social relations will obviously have different performance implications for embeddedness and social capital. In its simplest formulation, content of social ties may be considered by using Granovetter's (1973; 1974) construct of strength of ties.

Embeddedness in weak and strong social ties

Resources accessed and benefits accrued through strong and weak social ties may be distinct (Granovetter 1973; Bian 1997; Rowley et al. 2000). Strong social ties provide access to "fine-grained" information, allow transfer of tacit knowledge, and encrust social exchange with trust, respect, and emotional intensity (Granovetter 1973; Uzzi 1997; Bian 1997; Szreter 2002). Strong social ties are symmetric: the affinity among the connected individuals is generally mutual. In addition, networks of strong social relations, such as close kinship and friendship ties, often entail an obligation of reciprocity over a long time span (Polanyi 1957).

Weak social ties, such as infrequent contacts, provide access to useful information not generally available through an actor's strong ties (Granovetter 1973; Hansen 1999; Uzzi and Lancaster 2003). Weak social ties may or may not be symmetric. In addition, weak social ties connect actors to their contacts or acquaintances who are not close friends but with whom they may often transact in the economic sphere. In this sense, a non-anonymous business or knowledge transaction of an actor may be considered as a weak social tie (if it does not coincide with a strong social tie). ${ }^{16}$ More generally, all business (or knowledge) transactions that are not arms-length, impersonal, transactions of a 'pure' market may be treated as social ties and therefore be referred to as embedded ties (Uzzi 1996; 1997; Uzzi and Lancaster 2003).

A useful distinction between two different types of embedded ties may then be made: embeddedness of economic transactions in weak social relations and that in strong social relations. This distinction can be critical due to three reasons. First, as noted above, the content of weak social ties may be significantly different from strong

\footnotetext{
15 This is particularly true for economic exchange in modern developed economies but also in rural economies in developing countries of the type studied here. Historically, the degree of embeddedness in strong social bonds may have been different (see Polanyi 1944 [2001]).

16 A business or a knowledge network may then be viewed as a combination of weak and strong social ties. Note that strong social ties in these networks are the multi-stranded ones, i.e. they transmit knowledge (or another economic resource) in combination with the non-economic trust and social support. The social network of kinship and friendship ties here, however, is made up of strong ties only.
} 
social ties. As a result, economic transactions embedded in weak social ties may be characterized by a different type of 'social glue' as compared to those embedded in strong social ties (for example, mutual trust will more likely be associated with strongly embedded transactions). Second, this distinction highlights that implications of network structures, such as closure and structural holes, for economic performance of network members may be different for networks composed of predominantly weak or strong social ties. Third, strong social links are generally more durable than weak ones (see Podolny and Baron 1997); therefore, economic transactions embedded in strong social ties tend to be more stable in the long run than those embedded in weak social ties. Fourth, in technological evolution, weak embeddedness may be more important than strong embeddedness for accessing new knowledge at initial stages of a technology's evolution (Rowley et al. 2000; Cowan and Jonard 2006). ${ }^{17}$

Similarly, social capital derived from embeddedness in one's weak social ties may be distinct from that from strong social connections. In the literature on social capital however, social capital is generally strong social capital manifesting as trust, mutual respect and other features of strong social ties. Adopting this traditional definition of social capital, classification of different types of social capital can simply be based on the type of economic transactions. ${ }^{18}$ For example, an individual's knowledge flows embedded in her social ties can be used to measure social capital for knowledge. Similar measures of social capital for credit (or another economic resource) may be based on the number of social ties that act as conduits of credit. ${ }^{19}$ As emphasized earlier, this must be accompanied by a precise definition of the 'social relations' that provide access to knowledge or credit (which in the present study are close kinship and friendship ties).

In the remainder of this chapter, I focus on measuring the structural embeddedness of knowledge transactions in social networks constituted by strong ties.

17 This insight corresponds to the relative importance of network closure versus structural holes at different stages of technological evolution, if strong ties are assumed to be surrogates for closure and weak ties for structural holes.

18 This is important due to the wide variety in tie content. For example, network ties act as conduits of emotional support (Granovetter 1973); companionship and small favours (Wellman and Wortley 1990); provide access to information and other critical resources (Granovetter 1973; Burt 1992; Uzzi 1996); allow joint problem-solving to take place (Uzzi 1996); facilitate learning through transfer of knowledge (Inkpen and Tsang 2005; Podolny and Page 1998); permit sharing of risks and costs (Kogut 1988); allow cooptation and pooling of resources by partnering firms (Burt 1983a); and act as channels for exchange of goods and money in markets (DiMaggio and Louch 1998; McGinn and Keros 2002). Here I have grouped studies on interpersonal ties with those on inter-firm ties, though my focus is on the former.

${ }_{19}$ Note that this 'economic' interpretation of social capital does not preclude other interpretations where social capital may be primarily viewed in terms of trust or social support. I am simply arguing that there are many different types of social capital depending on the content of ties: if a set of ties is used to access non-economic resources, such as emotional support, then the size of such ties can be used to estimate social capital for emotional support. Also see footnote 11 above and the previous chapter on social capital. 
Corresponding results on (strong) social capital measurements are also presented. I use co-incidence between two networks to measure the degree of embeddedness. ${ }^{20}$ Both individual and network level measures of embeddedness (and social capital) are proposed.

\subsection{Measures of structural embeddedness}

I present two simple measures of the degree of embeddedness: the first determines the proportion of links in the knowledge network that also double as social (kinship and friendship) ties. The second measure is a correlation of the knowledge and the social networks based on Quadratic Assignment Procedure (QAP) for comparing networks (see Krackhardt 1987). ${ }^{21}$ The first measure of embeddedness simply tells us how much of the knowledge sharing actually takes place through the kinship and friendship ties of the social network. The second measure compares the structures of the two networks and tells us whether the two network structures are related to each other. The first one is sensitive to the density of the social network (the network in which the knowledge flows are embedded), whereas the second is not. I call the first measure simply embeddedness and the second correlation. I calculate both network- and individual-level measures of embeddedness but only the network level measure of correlation. $^{22}$

In addition, I run QAP-based regressions with the knowledge network as the dependent variable and the social network as the independent one. The same regression is also run with the business network as an independent variable.

\section{Embeddedness measures}

The proportion-based network level measures of embeddedness, for a network $\mathbf{K}$ in a network $\mathbf{S}$ is given by,

${ }^{20}$ I examine both network level and individual co-incidence. Coincidence in content is generally referred to as the multiplexity of a tie (Burt 1983b; Leffler et al. 1986; Marsden 1990). A multiplex tie is one through which an actor not only gets access to credit or knowledge, for example, but also emotional support. Multiplexity is sometimes viewed as an indicator of a strong tie, but strong ties with a single or diffuse content are also possible (Simmel 1950, cited in Granovetter 1973). Further, strong ties may be multiplex (or multi-dimensional) in the 'social' domain alone by acting as conduits for support, trust, confidence, and conviviality.

${ }^{21}$ For network data, the QAP-based correlation is preferred over traditional statistical measures that assume independence of individual observations. This is because network data is composed of dyads which are generally interdependent with each other, particularly those belonging to the same row and column of the connection matrix (i.e. connections of the same node in the network). For the same reason, in regressions on network data the QAP is the preferred over traditional statistical tests based on the OLS or the GLS approaches (further details are provided below; see Krackhardt 1988).

22 The QAP is generally used to compare two square matrices (the adjacency matrices of the two networks under comparison which contain all information on the structures of the two networks respectively). At the individual level, we only have a row and a column of size $\mathrm{N}$ (where $\mathrm{N}$ is the total number of nodes in the network). The row contains information on the outgoing links of an individual whereas the column contains information on her incoming links in the knowledge network. 


$$
E_{p}=\frac{\sum_{i, j} k_{i j} s_{i j}}{\sum_{i, j} k_{i j}}
$$

where $k_{i j}$ and $s_{i j}$ are elements of the adjacency matrices of the two networks. $\mathbf{K}$ is an asymmetric (directed) network whereas $\mathbf{S}$ can be asymmetric or symmetric. ${ }^{23}$ Note that $\mathrm{E}_{\mathrm{p}}$ only measures the embeddedness of $\mathbf{K}$ in $\mathbf{S}$ but not vice versa. The degree of embeddedness of $\mathbf{S}$ in $\mathbf{K}$ can be measured by changing the denominator in equation (9.1) to $\sum_{i, j} s_{i j}$.

At the individual level, the degree of embeddedness of an individual's ego-network $\mathrm{K}_{\mathrm{i}}$ in her corresponding network $\mathrm{S}_{\mathrm{i}}$ is given by,

$$
\begin{array}{rlr}
e_{p, i}= & \text { for outgoing links } \\
\sum_{j} k_{i j} k_{i j} & \\
e_{p, i}=\frac{\sum_{j} k_{j i} s_{j i}}{\sum_{j} k_{j i}} & \text { for incoming links }
\end{array}
$$

where $k_{i j}$ and $s_{i j}$ are elements of the adjacency matrices of the two networks $\mathbf{K}$ and $\mathbf{S}$. $\mathbf{K}$ is an asymmetric network. If $\mathbf{K}$ and $\mathbf{S}$ are knowledge and social networks respectively, $e_{p, i}$ in equation (9.2a) measures the (proportional) embeddedness of an individual's knowledge recipients in her social network, whereas $e_{p, i}$ in equation (9.2b) gives the proportion of an individual's knowledge sources who are also socially connected with her. In other words, using equations (9.3), I simply measure the share of an individual's incoming and outgoing knowledge links that also double as her social ties. $^{24}$

${ }^{23}$ The adjacency matrix of network $\mathbf{S}$ can have non-zero elements for direct links (one-step), as is the case for a simple social network. Or the adjacency matrix may have non-zero elements for indirect links (all paths of length two) in the same network. The network $\mathbf{K}$ here however is made up of direct links only.

${ }^{24}$ Conversely, an individual's social ties that provide access to knowledge give an indication of the individual's social capital for knowledge. Social capital for knowledge access is given by the number of social ties that coincide with incoming knowledge links (the numerator in equation $5.2 \mathrm{~b}$ ). Social capital for knowledge delivery, which is akin to power as a result of being a source of knowledge, is indicated by the number of social ties that coincide with outgoing knowledge links (the numerator in equation 9.2a). At the network level, social capital for knowledge is simply the number of coincident links between the knowledge and the social network (the numerator in equation 9.1). 


\section{Correlation measure}

The correlation measure compares two network structures to determine how similar they are to each other. ${ }^{25}$ I use the Quadratic Assignment Procedure (QAP) to compare the adjacency matrices of two networks (Krackhardt 1987; Borgatti et al. 2002). As discussed above, network data are not amenable to standard statistical correlation techniques because it suffers from the structural autocorrelation problem, which is circumvented by using the QAP (Krackhardt 1987). This structural autocorrelation problem arises due to the interdependence between the different dyads (or relationships) of a single node i.e., the different elements of the same row and the same column of the adjacency matrix. The QAP avoids this problem by using repeated permutations of the rows and columns of one of the two matrices under comparison. ${ }^{26}$ The second matrix is kept unchanged. In these permutations, if row 2 is interchanged with row 1 for example, then the same must be done to columns 2 and 1 of the adjacency matrix. After each permutation, the correlation or the 'measure of similarity' (Krackhardt 1987) is recalculated and stored. The average value of the correlation coefficient after a large number of permutations is compared to the observed correlation between the original matrices before the permutations. The observed correlation coefficient is significant if only a small percentage $(<5 \%)$ of the results after random permutations are as large (or as small) as the observed value.

Graphically, a permutation of corresponding rows and columns can be depicted as interchanging the labels of nodes in a graph. This switching of the node labels in a graph is illustrated using an example of two simple network structures (Krackhardt 1987): see Figures 9.1(a)-9.1(d) below. In Figure 9.1(a), the two network structures under comparison are drawn without node labels. In the first permutation, shown in Figure 9.2(b), the node labels are assigned and corresponding adjacency matrices are generated. If a node in a graph is labelled 1, then its relations (or dyads) occupy the first row and column of the graph's adjacency matrix. As we move from Figure 9.1(b) to the new permutation shown in 9.1(c), the label of the right hand corner node in graph (ii) for example changes from 3 to 4 . As a result of this change, the dyads of this node move from the third to the fourth row and column in the adjacency matrix of graph (ii). Note that only graph (ii) in the Figures undergoes any permutations. In this way, a large number of permutations (in the present example, a maximum of 120) can be performed on graph (ii) and the resulting adjacency matrix compared to the adjacency matrix of the fixed graph (i). The correlation coefficient is recalculated after each permutation. These calculations are based on a comparison of the corresponding elements (the dyads in the same cell location) in the two adjacency matrices.

\footnotetext{
25 A standard correlation measure is the Pearson correlation coefficient (which is generally defined in any elementary book on statistical analysis). In the procedure used in this chapter for determining the similarity between the knowledge and social (or business) networks, the correlation coefficient is calculated repeatedly using the Quadratic Assignment Procedure (QAP). The computer software program for social network analysis UCINET 6 is used to perform the QAP (Borgatti et al. 2002). ${ }^{26}$ The QAP may be considered as a permutation test for dyadic data (each observation is a dyad).
} 


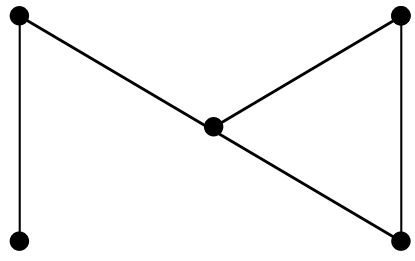

(i)

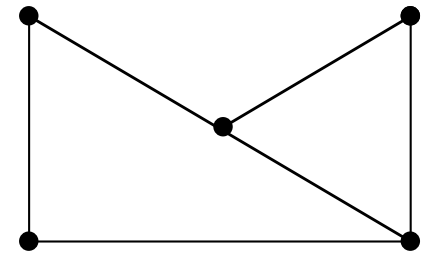

(ii)

Figure 9.1(a) An example of two simple network structures under comparison (Krackhardt 1987).

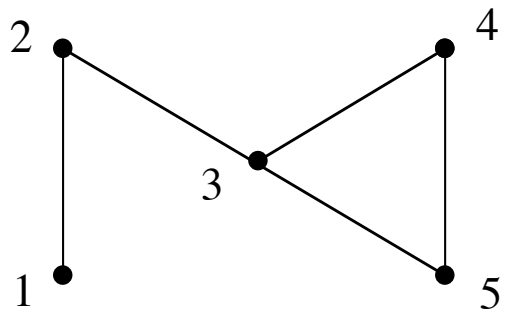

(i)

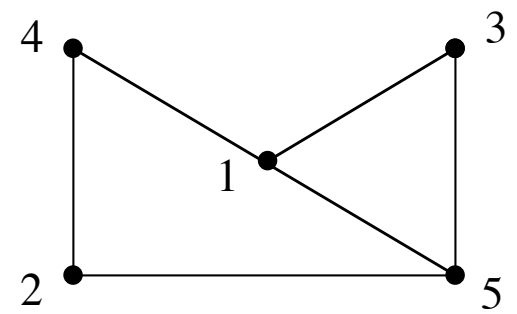

(ii)

Figure 9.1(b) The first permutation of the adjacency matrix of graph (ii) (Krackhardt 1987).

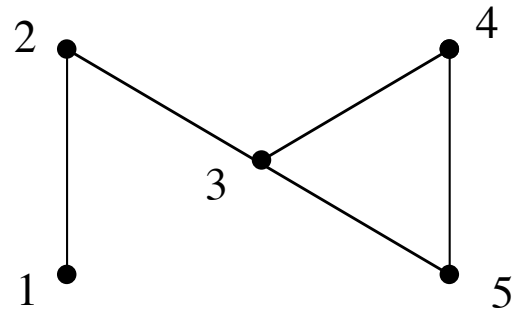

(i)

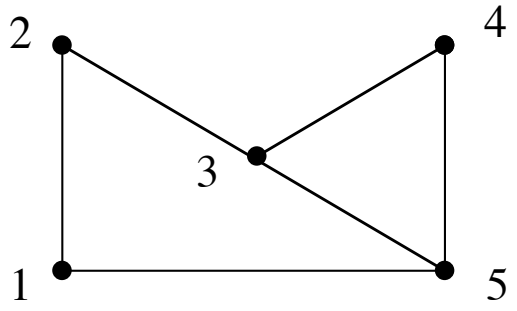

(ii)

Figure 9.1(c) The second permutation of the adjacency matrix of graph (ii) (Krackhardt 1987).

\section{Regression procedure}

Linear regressions with knowledge network as the dependent variable and the social or the business network as the independent variable are performed using the QAP, once again in UCINET 6 (Borgatti et al. 2002; Krackhardt 1987; 1988). The data points are the dyads of the knowledge network (dependent variable) and those of the social or business network (independent variable). The procedure of random permutations, of the rows and columns of the adjacency matrix of one of the graphs, in the regressions is the same as the one explained above for the correlation. The basic regression approach used in UCINET 6 is the Ordinary Least Squares (OLS) method which is performed repeatedly after every permutation of the dependent variable i.e., the adjacency matrix of the knowledge network in this case. 
In the following section, I use the measures defined in the foregoing to estimate individual and network level embeddedness. Below I start with the embeddedness of knowledge flows in the local innovation system, followed by measurement of embeddedness in the community of agricultural practitioners.

\subsection{Embeddedness of knowledge flows in the local innovation system}

In the vast literature on innovation systems at the national, sectoral, regional, and local levels, an innovation system is treated as a network of actors with diverse capabilities. ${ }^{27}$ I assume that the three networks of the village together constitute a local innovation system. Interactive learning through knowledge sharing and joint problemsolving among diverse actors forms the basis of sustainable knowledge creation and knowledge diffusion in an innovation system (see chapter 2 for a brief overview of the innovation systems literature). This interactive learning, underpinned by knowledge sharing and joint problem-solving, is geographically and socially embedded (see for example, Lundvall 1992; Lundvall et al. 2002; Asheim and Coenen 2005). According to Lundvall et al. (2002: 225), "learning and innovation are interactive processes, which depend on trust and other elements of social cohesion." They reaffirm, "production of intellectual capital (learning) is strongly dependent on social capital." Lundvall et al. (2002: 225). However, the way in which this dependence or relationship between social capital (embeddedness) and learning operates is rarely specified in the innovation systems literature. Since learning is an interactive activity in an innovation system, it depends on knowledge flows among the actors that form this system. In this section, I examine the degree to which knowledge flows are embedded in direct and indirect social relations characterized by "trust, and other elements of social cohesion."

The present discussion may also be viewed as an attempt to connect cognitive coordination with socio-political coordination in an innovation system. An effective mix of these two types of coordination makes interactive learning and innovation successful (see Coriat and Weinstein 2002 for this argument for learning in a firm). In this vein, the following is an attempt to examine the interpenetration between cognitive coordination, represented by the knowledge network of the village, and the socio-political coordination through the social network. ${ }^{28}$ This interpenetration of knowledge and social networks is first investigated using the network-level embeddedness measure and the QAP correlation coefficient defined in the previous

\footnotetext{
${ }^{27}$ In addition of course, there are the formal and informal institutions (Lundvall et al. 2002; Parto et al. 2005). Actors from the formal institutions are included in the innovation system as a network. Informal institutions such as norms and habits partly enter the picture through the content of social ties in the network. A fuller institutional account entails an analysis of how norms and habits constrain and facilitate human agency over time in an innovation system.

28 See the previous chapter for a discussion on socio-political coordination in the village's social network. The focus of my work here is admittedly narrower than institutional focus of Coriat and Weinstein (and other 'institutionalists' in the innovation system literature).
} 
section. Subsequently I perform regressions to test the hypothesis that social cohesion (a dense social network of strong ties) promotes effective transfer of knowledge in a local innovation system. I refer to this hypothesis as the SN hypothesis. The QAPbased regression assesses the predictability of the knowledge-flow network if the structure of the social network among the same group of people is known i.e., the knowledge network is the dependent variable and the social network of strong ties is the independent one.

\section{Network-level social embeddedness}

Embeddedness of knowledge flows is measured in two types of social ties here: direct and indirect (two-step) ties. The first measures access of knowledge directly from one's friends and kin, and the second from friends of friends (or kin of friends, etc.). ${ }^{29}$ The networks compared have 283 nodes each, and are constituted by Punukula residents plus all their business and knowledge contacts (see Chapter 5 for more on network data and approach used to collect the data). I call this set of networks the 'undivided' networks, which together make up the local innovation system.

The degree of embeddedness, measured using equation (9.1), for the knowledge network in social network of direct links is 0.0970 . That is, only $9.70 \%$ of the links (32 out of a total of 330) in the knowledge network are embedded in direct social ties of close friendship and kinship. ${ }^{30}$ Embeddedness in indirect links of the social network is 0.1970 , i.e. $19.70 \%$ (65 out of 330) of the total knowledge flows take place among friends of friends. These results suggest that, in this local innovation system, only a small percentage of knowledge flows are embedded in social relations characterized by trust and other facets of social cohesion.

The greater value of embeddedness in indirect social ties, as compared to direct social ties, may simply be due to the greater density of the social network of indirect links (friends of friends). This density is almost four times that for direct links: 1954 and 529 links respectively. The dependence of embeddedness results on social network-density is avoided by calculating QAP-based correlation coefficient between the same two networks. The QAP correlation between the knowledge network and the network of direct kin/friends is 0.047 ( $p<0.001)$, as compared to $0.044(p<0.001)$ for correlation of the knowledge network with the network of friends of friends. ${ }^{31}$ These low values suggest that the structure of the social network is very different from the knowledge network in this local innovation system. The social ties through which

\footnotetext{
29 Note that embeddedness in direct and indirect links may also be viewed as a measure of embeddedness of knowledge links within a clique of the social network (a social group based on subcaste or lineage): here I am assuming that most members of the same clique are separated from each other by short paths (of length equal to one or two). A more precise measurement of sub-caste based division of knowledge flows must be preceded by the identification of the community structure, or cliques, in the social network.

30 Social capital for knowledge is simply reflected in the number of coincident ties (32 out of a total of 529 ties in the social network).

31 The QAP correlation results are based on 5000 permutations, run in UCINET 6 (Borgatti et al. 2002).
} 
knowledge flows, therefore, are distributed sparsely across the social network, as shown in Figure 9.2a for the social network of direct ties (see Giuliani 2005 for similar results in wine cluster settings). ${ }^{32}$ Therefore, effective socio-political coordination in a local innovation system as evidenced by a dense social network (such as the one presented here) does not seem to act as impetus to effective knowledge transfer among the same group of people. I confirm this result using the regressions later.

Table 9.1 Embeddedness and social capital for knowledge in the local innovation system

\begin{tabular}{|l|l|l|l|l|}
\hline $\begin{array}{l}\text { Path length of social } \\
\text { ties }\end{array}$ & $\begin{array}{l}\text { Density } \\
\text { of social } \\
\text { network }\end{array}$ & $\begin{array}{l}\text { Number of } \\
\text { embedded } \\
\text { knowledge flows }\end{array}$ & $\begin{array}{l}\text { Embedded- } \\
\text { ness }\end{array}$ & $\begin{array}{l}\text { QAP } \\
\text { Correlation }\end{array}$ \\
\hline $\begin{array}{l}\text { Direct social ties (close } \\
\text { kin and friends) }\end{array}$ & 0.0133 & 32 & 0.0970 & 0.047 \\
\hline $\begin{array}{l}\text { Indirect ties (friends of } \\
\text { friends) }\end{array}$ & 0.0490 & 65 & 0.1970 & 0.044 \\
\hline
\end{tabular}
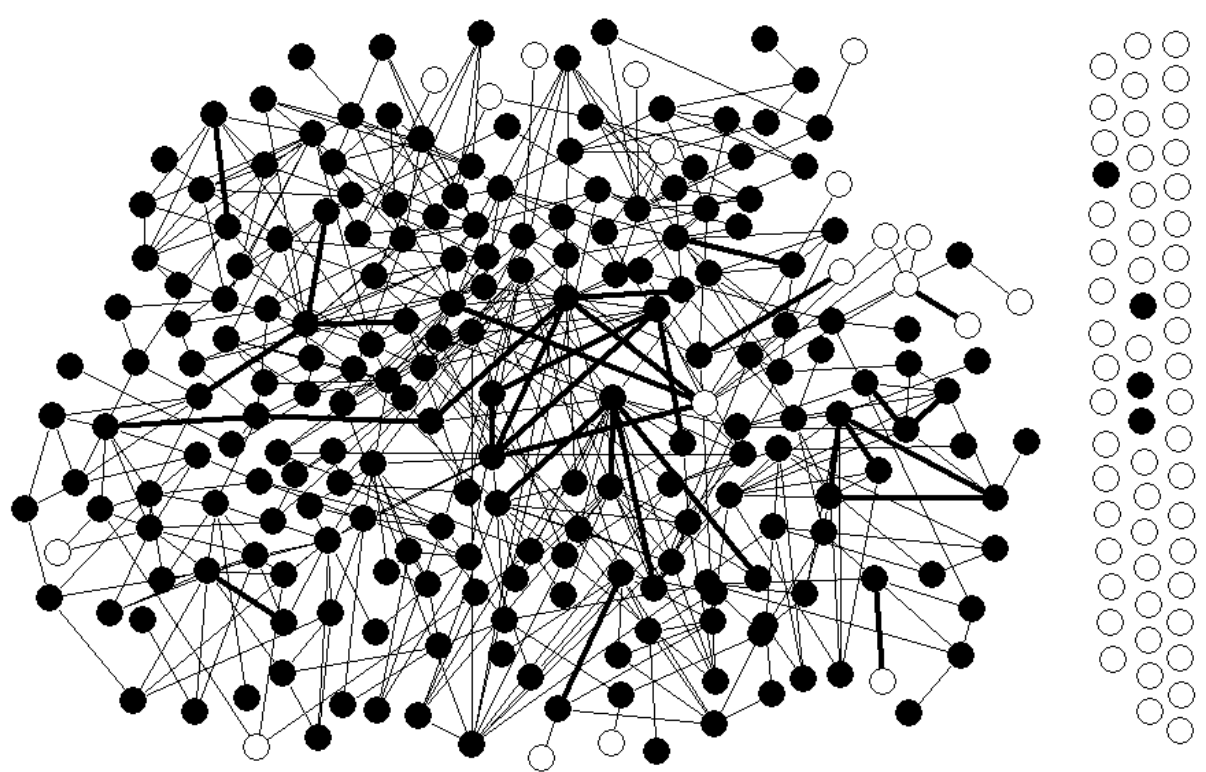

Figure 9.2a Knowledge flows embedded in the social network (social ties which transfer knowledge are represented as thicker in black). Black nodes represent inhabitants of the village whereas the white nodes depict outsiders. Isolates are shown at the right of the figure.

32 The 'inverse' of this Figure is shown in Figure 9.2b where social ties are overlaid on the knowledge network. Figure 9.2b reaffirms that few knowledge links are buttressed by social ties: most of these socially buttressed knowledge links are farmer-to-farmer and a few from pesticide dealer-to-farmer but none between the NGO representatives and the farmers. 


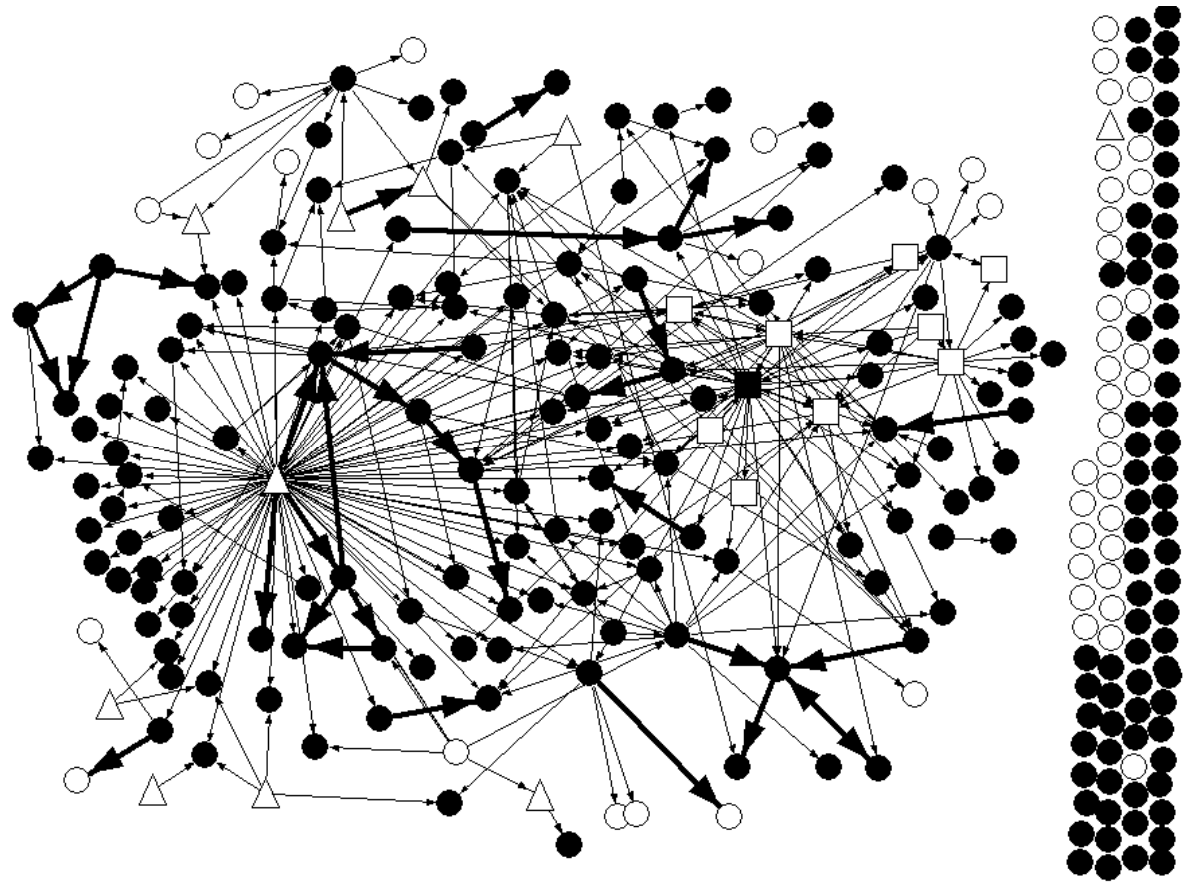

Figure 9.2b Friendship and kinship ties overlaid on the knowledge network (knowledge links buttressed by the social ties are thicker). Black nodes depict Punukula inhabitants and white ones outsiders. Triangles depict pesticide dealers whereas squares represent NGO representatives.

The QAP regression results of the knowledge network on the social network as the independent variable are shown in Table 9.2. In model 1, the independent variable is the social network of direct ties, whereas in model 2 it is the same network with indirect ties. Once again the results in the Table are based on 5000 QAP permutations in UCINET 6 (Borgatti et al. 2002). As expected, considering the poor correlation results discussed above, the value of the $\beta$ coefficient is very low. ${ }^{33}$ And the regression fit for both social networks (direct and indirect links) is very poor $\left(\mathrm{R}^{2}=0.02\right)$. The predictability of the knowledge network using the regression coefficients and a map of strong social ties is therefore very poor. I find little evidence to support the SN hypothesis defined above.

Table 9.2 Regression of the knowledge flow network on the social network of the village

\begin{tabular}{|l|l|l|}
\hline Model & $\underline{\underline{1}}$ (direct social ties) & $\underline{\underline{2} \text { (indirect social ties) }}$ \\
\hline $\boldsymbol{\beta}$ & $0.026^{*}$ & $0.013^{*}$ \\
\hline Intercept & 0.0038 & 0.0035 \\
\hline
\end{tabular}

33 The results for the regression coefficients shown in Table 9.2 are unstandardized values. The standardized values simply equate $\beta$ to correlation coefficients of Table 9.1 and the intercept to zero. 


\begin{tabular}{|l|l|l|}
\hline $\mathrm{R}^{2}$ & 0.02 & 0.02 \\
\hline${ }^{*} \mathrm{p}<0.001$ &
\end{tabular}

\section{Individual-level embeddedness in social ties}

At the individual-level, results on the embeddedness of incoming and outgoing flows of knowledge are presented separately. The degree of embeddedness of incoming and outgoing knowledge flows assesses the 'social' foundations of knowledge access and delivery by an individual. Only 26 and 24, of the 283 networkmembers have any incoming and outgoing knowledge links that coincide with their direct social ties respectively. Results for the 26 individuals who have one or more socially embedded incoming knowledge flows are shown in Table 9.3. The second column shows the number of incoming links embedded in social ties (which can also be used as a measure of an individual's social capital for knowledge access); and the third column lists the degree of embeddedness of an individual's incoming knowledge flows. All individuals listed in Table 9.3 are Punukula residents unless otherwise stated in column I. All names listed are pseudonyms.

As shown in Table 9.3, two people have the highest number of 3 incoming knowledge links that are embedded (see column II). They have the highest absolute embeddedness (the total number of knowledge links that are embedded in social ties) and therefore possess the highest social capital for knowledge access in the network. This result seems consistent with the status of the two individuals in the village: they are headmen of their respective sub-castes in the village; and are members of the dominant core of the social network (as discussed in Chapter 8). In all, five out of ten people from the dominant core of the social network of the village have non-zero values of social capital for knowledge access.

The two individuals with the highest social capital, Gorinta, EG and Koli, ED, do not however have the highest degree of embeddedness in the network, which means that not all of their knowledge links are embedded in social ties. In all eight people have the maximum possible value, 1, for the degree of embeddedness i.e., all knowledge links of these people are embedded in direct social ties. This means that only $2.8 \%$ of the network members (8 out of 283) access all their (pest control related) knowledge from their strong social (kinship and friendship) ties. All but one of these people have only 1 incoming knowledge link. And all but one of them also act as knowledge sources for one of their kin/friends (see Table 9.4 below).

Two conclusions can be drawn from the detailed individual level results, which confirm the network level results. First, very few people (approximately 9\%) in the local innovation system access knowledge from their kin and friends. Therefore, the relatively dense social network of kin and friends does not act provide a fertile social substratum for knowledge exchange in the system. Second, even individuals with one or more embedded knowledge links do not access knowledge from all people in their social network (see column IV in Table 9.3 for their degree in the social network). An individual's knowledge access links, even when embedded, are scattered across her 
(denser) social network. Thus, despite the possibility of frequent interaction and similarity in profession among the kin and friends (agriculture), which for example is considered important for knowledge exchange because it reduces the cognitive distance between the recipient and the source, knowledge flows over the social network are found to be sparsely dispersed, rather than densely uniform.

Table 9.3 Individual embeddedness of incoming knowledge links in direct social ties - 'undivided' networks

\begin{tabular}{|c|c|c|c|c|}
\hline & I & II & III & IV \\
\hline & $\begin{array}{l}\text { Name of the } \\
\text { individual }\end{array}$ & $\begin{array}{l}\text { Number of } \\
\text { incoming links } \\
\text { coincident with } \\
\text { social ties }\end{array}$ & $\begin{array}{l}\text { Degree of } \\
\text { embedded- } \\
\text { ness }\end{array}$ & $\begin{array}{l}\text { Number of } \\
\text { ties (degree) } \\
\text { in the social } \\
\text { network }\end{array}$ \\
\hline 1. & Gorinta, EG & 3 & 0.75 & 16 \\
\hline 2. & Koli, ED & 3 & 0.5 & 16 \\
\hline 3. & Lal, BE & 2 & 1 & 5 \\
\hline 4. & Pillai, GC & 2 & 0.667 & 3 \\
\hline 5. & Lal, BM & 1 & 1 & 7 \\
\hline 6. & Chet, DL & 1 & 1 & 4 \\
\hline 7. & Pillai, GA & 1 & 1 & 4 \\
\hline 8. & Joardar, GX & 1 & 1 & 10 \\
\hline 9. & Palam, HS & 1 & 1 & 3 \\
\hline 10. & $\begin{array}{l}\text { Lal, AG, } \\
\text { Bhagwativagu }\end{array}$ & 1 & 1 & 1 \\
\hline 11. & $\begin{array}{l}\text { Romi, AC, } \\
\text { Kamalpur }\end{array}$ & 1 & 1 & 1 \\
\hline 12. & $\begin{array}{l}\text { Chiruvella, P, } \\
\text { Victory } \\
\text { Pesticides }\end{array}$ & 1 & 0.5 & 4 \\
\hline 13. & Koli, EA & 1 & 0.5 & 12 \\
\hline 14. & Koli, EB & 1 & 0.5 & 10 \\
\hline 15. & Navya, EL & 1 & 0.5 & 9 \\
\hline 16. & Sapna, FC & 1 & 0.5 & 9 \\
\hline 17. & Yarla, FU & 1 & 0.5 & 6 \\
\hline 18. & Pillai, FZ & 1 & 0.5 & 8 \\
\hline 19. & Velu, HE & 1 & 0.5 & 10 \\
\hline 20. & Subba, CE & 1 & 0.333 & 5 \\
\hline 21. & Navya, ES & 1 & 0.333 & 6 \\
\hline 22. & Krithi, HB & 1 & 0.333 & 4 \\
\hline 23. & Yarla, FV & 1 & 0.25 & 6 \\
\hline 24. & Sapna, EW & 1 & 0.2 & 7 \\
\hline
\end{tabular}




\begin{tabular}{|l|l|l|l|l|}
\hline 25. & Veena, IE & 1 & 0.2 & 10 \\
\hline 26. & Naidu, CY & 1 & 0.167 & 8 \\
\hline
\end{tabular}

Results for the 24 individuals with non-zero values of embeddedness of outgoing knowledge flows are listed in Table 9.4. Three people, BJ Lal, HE Velu, and M Raj, have three embedded knowledge links each and thus possess the highest social capital due to knowledge delivery. One of these three people is the main pesticide supplier to the farmers in the village: as discussed in chapter 6 , he has the highest out-degree centrality in the knowledge network. The other two are farmers from the village. HE Velu is the president of the village council. He is also a member of the dominant core of the village's social network, as discussed in chapter 8. Finally, BJ Lal is one of three farmers who experimented successfully with NPM, which as discussed in section 6.2 is a critical factor in the innovation capacity of farmers. ${ }^{34}$ As shown in the third column, ten people have the maximum value of 1 of proportional embeddedness of outgoing knowledge links. All but two of these 10 people have only 1 outgoing knowledge link.

In Table 9.4, people use a small proportion of their social ties for transmitting knowledge (compare column II to column IV). The social network of the people is clearly denser than their knowledge delivery networks.

Only ten people (out of 24) are common to Tables 9.3 and 9.4. Thus, while in principle an individual with high social capital for knowledge access may also have high social capital for knowledge delivery, the two forms of social capital cannot be equated. ${ }^{35}$ Social capital for knowledge access shows how well an individual is situated in her social network to access knowledge for agricultural pest control: livelihood creation/protection through resource access is the focus here. The delivery side of social capital is akin to power: it refers to an individual's potential control over others' livelihoods through provision of a useful resource for productive activities.

Table 9.4 Individual embeddedness - outgoing knowledge links in direct social ties

\begin{tabular}{|l|l|l|l|}
\hline & I & II & III \\
\hline & $\begin{array}{l}\text { Name of the } \\
\text { individual }\end{array}$ & $\begin{array}{l}\text { No. of outgoing } \\
\text { links coincident } \\
\text { with social ties }\end{array}$ & $\begin{array}{l}\text { Degree of } \\
\text { embedded- } \\
\text { ness }\end{array}$ \\
\hline 1. & Lal, BJ & 3 & 1 \\
\hline 2. & Velu, HE & 3 & 0.75 \\
\hline
\end{tabular}

\begin{tabular}{|l|} 
IV \\
\hline $\begin{array}{l}\text { Degree in } \\
\text { the social } \\
\text { network }\end{array}$ \\
\hline 8 \\
\hline 10 \\
\hline
\end{tabular}

\footnotetext{
${ }^{34}$ Note how social capital for knowledge, as in the case of these two farmers, is correlated with technological and/or social capability. More generally, the nature of economic and social outcomes may be influenced by economic and non-economic factors. Interdisciplinary concepts such as embeddedness and social capital permit the interpenetration of technological, economic, and noneconomic (social) factors in social scientific explanations.

35 Having high values of both forms of social capital may translate into some form of prominence in a network. For example, four of the ten people common to the two lists are members of the dominant core of the social network discussed in Chapter 8.
} 


\begin{tabular}{|l|l|l|l|}
\hline 3. & $\begin{array}{l}\text { Raj, M, A-One } \\
\text { Fertilizers }\end{array}$ & 3 & 0.038 \\
\hline 4. & Koli, ED & 2 & 1 \\
\hline 5. & Sapna, FC & 2 & 0.5 \\
\hline 6. & Subba, CD & 1 & 1 \\
\hline 7. & Naidu, CX & 1 & 1 \\
\hline 8. & Koli, EA & 1 & 1 \\
\hline 9. & Sapna, EY & 1 & 1 \\
\hline 10. & Yarla, FV & 1 & 1 \\
\hline 11. & Pillai, FZ & 1 & 1 \\
\hline 12. & Palam, HV & 1 & 1 \\
\hline 13. & Palam, HY & 1 & 1 \\
\hline 14. & $\begin{array}{l}\text { Sagi, U, ChemCorp, }, \\
\text { Hyderabad }\end{array}$ & 1 & 0.5 \\
\hline 15. & Lal, BM & 1 & \\
\hline 16. & Sree, FI & 1 & 0.5 \\
\hline 17. & Muthu, GD & 1 & 0.5 \\
\hline 18. & Udit, IO & 1 & 0.5 \\
\hline 19. & Kota, DN & 1 & 0.5 \\
\hline 20. & Pillai, GA & 1 & 0.333 \\
\hline 21. & Gorinta, EG & 1 & 0.333 \\
\hline 22. & Sapna, EW & 1 & 0.2 \\
\hline 23. & Nath, FG & 1 & 0.2 \\
\hline 24. & Yarla, FQ & 1 & 0.2 \\
\hline & & & 0.063 \\
\hline
\end{tabular}

\begin{tabular}{|l|}
\hline 8 \\
\hline 16 \\
\hline 9 \\
\hline 6 \\
\hline 12 \\
\hline 4 \\
\hline 6 \\
\hline 8 \\
\hline 12 \\
\hline 4 \\
\hline 1 \\
\hline 7 \\
\hline 3 \\
\hline 4 \\
\hline 3 \\
\hline 2 \\
\hline 4 \\
\hline 16 \\
\hline 7 \\
\hline 6 \\
\hline 9 \\
\hline
\end{tabular}

To summarize, a high degree of social embeddedness of knowledge flows, which facilitates learning in an innovation system, was not observed. The vast majority of knowledge flows take place among farmers and 'experts' who are generally not socially tied with each other (either directly or indirectly). ${ }^{36}$ Conversely, the farmers' extensive networks of social ties do not act as a foundation for knowledge sharing and learning. ${ }^{37}$ In other words, social capital through strong ties characterized by trust and solidarity does not play a significant role in the provision of knowledge in this local innovation

\footnotetext{
${ }^{36}$ For results on individual embeddedness in indirect social ties, see Appendix D of the thesis. Those results simply restate the patterns observed for embeddedness in direct social ties.

37 As discussed in chapter 6, a large percentage of knowledge exchanges of farmers take place with contacts residing outside the village. These outsiders, such as the pesticide dealers and NGO representatives, are sparsely connected in the social network of the village. Only $31(5.86 \%)$ out of a total of 529 links in the social network involve an actor residing outside Punukula. Barring a few exceptions, these sparse social ties between the outside actors and the farmers do not act as conduits of knowledge.
} 
system. So, the present case is a counter-example to the hypothesis that social ties provide an effective foundation for knowledge sharing in innovation systems. ${ }^{38}$

\section{Sourcing knowledge from business contacts in the local innovation system}

User-producer interactions along the production chain of a good form a cornerstone of the innovation systems framework (see for example, Lundvall 1985; Lundvall 1988 for early studies highlighting the centrality of user-producer interactions in technological learning). This interactive learning and knowledge sharing occur between actors connected through business ties such as those between buyers and suppliers. In this section, I investigate the interpenetration of knowledge flows and business relations in the local innovation system. This degree of embeddedness of knowledge flows in the business network of the village is calculated in the same way as social embeddedness in the previous section.

In addition, here I test the hypothesis that the network of user-producer (or buyersupplier) business transactions forms the basis for knowledge sharing in the local innovation system. I term this the $\mathrm{BN}$ hypothesis. This hypothesis is tested using the QAP-based correlation coefficient and regression of the knowledge network on the business network.

The business network under consideration here is constructed around the pest control activities of Punukula farmers (procurement of pesticides and NPM materials). Studying the coincidence of knowledge flows with these supplier-buyer transactions allows a direct analysis of the importance of user-producer interactions in this local innovation system. I begin with network level measures and regressions, followed by a discussion about the individual-level measures of embeddedness.

Values of embeddedness of knowledge flows in the business network for accessing pesticides and NPM materials are shown in Table 9.5. See Figure 9.3 for a map of the business network where ties that also transfer knowledge are highlighted. Seventy-one knowledge links out of a total of $330(21.5 \%)$ are embedded in this business network. ${ }^{39}$ The proportional embeddedness of knowledge in the business network is much greater than the corresponding embeddedness in the social network (0.097, see Table 9.1). In addition, the level of correlation found between the knowledge and business networks

\footnotetext{
38 Whether this knowledge sharing in an innovation systems leads to learning and innovation or not is another issue, which was addressed in Chapter 6. There I found that learning and real innovation in this system was very limited despite the presence of the largely 'top-down' knowledge transfer links. However, despite the limited innovation success, Punukula received wide media coverage for its farmers' achievements. This latter success in controlling the representation of pest-control events in the village was studied in Chapter 8 .

39 An estimate of reverse embeddedness may be illustrative here: that of the business network of pest control in the knowledge network. As the density of the business network for pest control is smaller than that for the knowledge network, the reverse (proportional) embeddedness value due to the 71 coincident links is higher at 0.5868 i.e. $58.68 \%$ of a total of 112 business links are embedded in the knowledge network. In other words, a large percentage of buyer-supplier transactions in the business network double as user-producer interactions transferring useful knowledge.
} 
for pest control is substantially greater than that between the knowledge and social networks $(0.351$ vs. 0.047 , both at $\mathrm{p}<0.001)$. Thus, a larger number of knowledge flows are embedded in the business network than in the social network; and the structure of the knowledge network possesses greater similarity to that of the business network. Thus, we find reasonable evidence that the business network of direct userproducer interactions acts as the basis for knowledge sharing in the local innovation system.

The calculation of the correlation coefficient between the knowledge and business networks is limited to direct links alone. Since the business network for pest control only includes one step of the agricultural production chain, no indirect links exist in the network. As a result, embeddedness of knowledge links in indirect business ties is not possible here.

Table 9.5 Embeddedness of knowledge flows in the business network for pest control

\begin{tabular}{|l|l|l|l|}
\hline Path length of business ties & $\begin{array}{l}\text { No. of embedded } \\
\text { knowledge flows }\end{array}$ & Embeddedness & $\begin{array}{l}\text { QAP } \\
\text { Correlation }\end{array}$ \\
\hline Direct business relations & 71 & 0.2152 & 0.351 \\
\hline Indirect business relations & 0 & 0 & - \\
\hline
\end{tabular}

For the knowledge network regression on the business network, results are displayed in Table 9.6 below. I refer to this regression as model 3. Again the results are based on 5000 QAP permutations in UCINET 6 (Borgatti et al. 2002). The present regression is a much better fit than that with the social network of the village $\left(\mathrm{R}^{2}=0.123\right.$ as compared to 0.02$)$. Therefore, the predictability of knowledge transfers is much more plausible using information of the business network structure of the community rather than its social network structure. This result confirms the correlation result shown in Table 9.5 that the business network forms an effective substratum for knowledge transfer and thus the BN hypothesis is supported by the evidence presented here.

Table 9.6 Regression of the knowledge flow network on the business network

\begin{tabular}{|l|l|}
\hline Model & $\underline{3}$ (direct business ties) \\
\hline$\beta$ & $0.558^{*}$ \\
\hline Intercept & 0.0033 \\
\hline $\mathrm{R}^{2}$ & 0.123 \\
\hline
\end{tabular}

${ }^{*} \mathrm{p}<0.001$ 


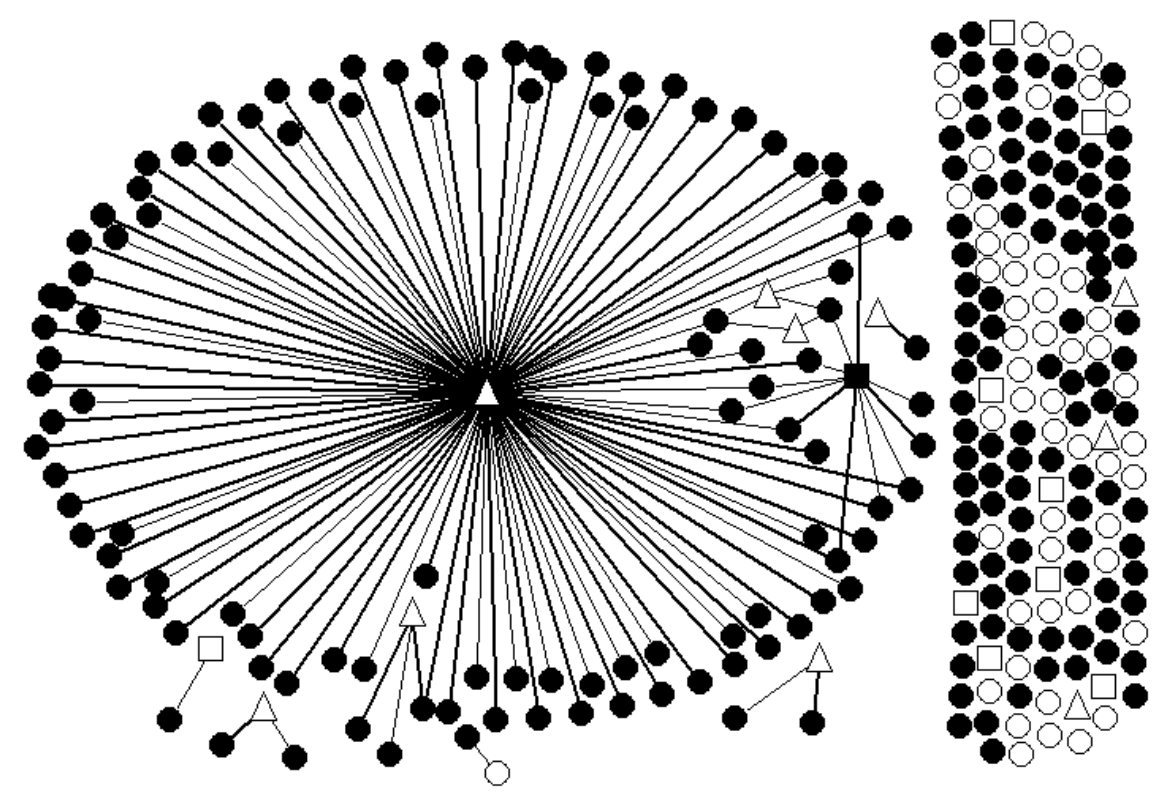

Figure 9.3 Knowledge flows in the business network (business ties which transfer knowledge are represented as thicker in black, remaining ties are in grey). All-in-one dealers are represented as triangular nodes whereas the NGO representatives are shown as squares ones. Black nodes represent village inhabitants and white ones depict outsiders.

At the individual level, actors with at least one incoming (outgoing) knowledge link that is embedded in corresponding business ties number 67 (6). Names of fifty people with at least one incoming knowledge link that is embedded in a corresponding business tie are listed in Table 9.7. Very few individuals (only 3) have more than one incoming knowledge link that doubles as a business relation. This is largely because few people had more than 1 or 2 business links for procuring pesticides (see column IV). Of the 67 actors with one embedded link, 27 with the maximum degree of embeddedness take problem-solving advice only from their pesticide (or NPM input) supplier. The remaining 40 farmers possess at least one incoming knowledge link that is not from their pest-control material supplier.

Table 9.7 Individual embeddedness of incoming knowledge links in direct business network for pest control

\begin{tabular}{|l|l|l|l|l|l|}
\hline & I & II & III & IV \\
\hline $\begin{array}{l}\text { Name of the } \\
\text { individual }\end{array}$ & $\begin{array}{l}\text { Number of incoming } \\
\text { knowledge flows coincident } \\
\text { with business ties }\end{array}$ & $\begin{array}{l}\text { Degree of } \\
\text { embedded- } \\
\text { ness }\end{array}$ & $\begin{array}{l}\text { In-degree in } \\
\text { the business } \\
\text { network }\end{array}$ \\
\cline { 1 - 3 } 1. & Suji, FM & 2 & 0.667 \\
\hline 2. & Joardar, GY & 2 & 0.4 & 2 \\
\hline
\end{tabular}




\begin{tabular}{|c|c|c|c|c|}
\hline 3. & Veena, IE & 2 & 0.4 & 2 \\
\hline 4. & Seri, AX & 1 & 1 & 1 \\
\hline 5. & Seri, AZ & 1 & 1 & 1 \\
\hline 6. & Subba, CD & 1 & 1 & 2 \\
\hline 7. & Jali, CR & 1 & 1 & 1 \\
\hline 8. & Sagi, EI & 1 & 1 & 1 \\
\hline 9. & Sapna, EX & 1 & 1 & 1 \\
\hline 10. & Sapna, EY & 1 & 1 & 1 \\
\hline 11. & Sapna, EZ & 1 & 1 & 1 \\
\hline 12. & Sapna, FA & 1 & 1 & 1 \\
\hline 13. & Sapna, FE & 1 & 1 & 1 \\
\hline 14. & Nath, FH & 1 & 1 & 1 \\
\hline 15. & Sree, FI & 1 & 1 & 1 \\
\hline 16. & Subbarao, FK & 1 & 1 & 1 \\
\hline 17. & Yarla, FR & 1 & 1 & 1 \\
\hline 18. & Muthu, GD & 1 & 1 & 1 \\
\hline 19. & Ray, GK & 1 & 1 & 1 \\
\hline 20. & Joardar, GX & 1 & 1 & 1 \\
\hline 21. & Velu, HC & 1 & 1 & 1 \\
\hline 22. & Velu, HF & 1 & 1 & 1 \\
\hline 23. & Velu, HG & 1 & 1 & 1 \\
\hline 24. & Velu, HK & 1 & 1 & 1 \\
\hline 25. & Palam, HY & 1 & 1 & 1 \\
\hline 26. & Palam, IB & 1 & 1 & 1 \\
\hline 27. & Veena, IF & 1 & 1 & 1 \\
\hline 28. & Veena, IG & 1 & 1 & 1 \\
\hline 29. & Yeluri, IN & 1 & 1 & 1 \\
\hline 30. & Udit, IO & 1 & 1 & 1 \\
\hline 31. & Seri, AR & 1 & 0.5 & 1 \\
\hline 32. & Seri, AW & 1 & 0.5 & 1 \\
\hline 33. & Lal, BL & 1 & 0.5 & 2 \\
\hline 34. & Datla, BW & 1 & 0.5 & 1 \\
\hline 35. & Subba, CA & 1 & 0.5 & 1 \\
\hline 36. & Subba, CB & 1 & 0.5 & 1 \\
\hline 37. & Subba, CC & 1 & 0.5 & 2 \\
\hline 38. & Gowda, CM & 1 & 0.5 & 1 \\
\hline 39. & Pal, DB & 1 & 0.5 & 2 \\
\hline 40. & Goli, DK & 1 & 0.5 & 1 \\
\hline 41. & Kotla, DO & 1 & 0.5 & 1 \\
\hline 42. & Koli, DW & 1 & 0.5 & 1 \\
\hline
\end{tabular}




\begin{tabular}{|l|l|l|l|}
\hline 43. & Koli, EB & 1 & 0.5 \\
\hline 44. & Sapna, EU & 1 & 0.5 \\
\hline 45. & Subbarao, FJ & 1 & 0.5 \\
\hline 46. & Arayar, GO & 1 & 0.5 \\
\hline 47. & Joardar, GW & 1 & 0.5 \\
\hline 48. & Velu, HE & 1 & 0.5 \\
\hline 49. & Seri, AU & 1 & 0.3333 \\
\hline 50. & Datla, BV & 1 & 0.3333 \\
\cline { 1 - 2 }
\end{tabular}

The six individuals with an outgoing knowledge link that is coincident with an outgoing business tie are listed in Table 9.8. Five of these six people are pesticide suppliers (the all-in-one dealers discussed in the previous chapters), and one is an NGO representative. Note the total absence of farmers as sources of knowledge embedded in the business network. This is because the outgoing links of the farmers to the crop buyers are not included in the one-step business network considered here (backward linkage to suppliers of pest control only). ${ }^{40}$

The main pesticide supplier to the village, M. Raj, tops the list with 62 outgoing knowledge links that are embedded in the outgoing business ties. This result highlights the 'dual domain' power of M. Raj: his position in the business network as the main pesticide supplier to the village is buttressed by his power in the knowledge network (as the most prominent source of problem solving knowledge). The second person in the list is the local SECURE representative living in the village with only 4 outgoing knowledge links that are embedded in her business links as a supplier of NPM materials to the farmers. ${ }^{41}$ The large difference in the degree of embeddedness between the pesticide supplier and the NGO representative supports the arguments concerning the control of the pesticide supplier, as the central member of dominant cores in the knowledge and business networks, on the agricultural activities of Punukula farmers presented in chapters 6 and 7.

Table 9.8 Individual embeddedness of outgoing knowledge links in direct business network for pest control

\begin{tabular}{|l|l|l|l|l|l|}
\hline & I & II & III & IV \\
\hline Name of the individual & $\begin{array}{l}\text { Number of } \\
\text { outgoing }\end{array}$ & $\begin{array}{l}\text { Degree of } \\
\text { embedded- }\end{array}$ & $\begin{array}{l}\text { Out-degree } \\
\text { in the }\end{array}$ \\
\cline { 1 - 3 }
\end{tabular}

40 See Appendix D for results on embeddedness of knowledge flows in the complete business network that includes the outgoing flows to the crop buyers. There are four farmers in that list of 11 people with embedded outgoing knowledge flows.

${ }^{41}$ Farmers collected most NPM inputs themselves and did not need to buy them from the NGO's or the pesticide dealers. To encourage new farmers to start using NPM, the NGO supplied the main NPM inputs such as neem to the farmers for some time. However, the number of business links in the present network used to procure NPM materials is relatively small (and much smaller than the number of links used to procure pesticides, see chapter 7). 


\begin{tabular}{|c|c|c|c|c|}
\hline & & $\begin{array}{l}\text { knowledge flows } \\
\text { coincident with } \\
\text { business ties }\end{array}$ & ness & \begin{tabular}{|l} 
business \\
network
\end{tabular} \\
\hline 1. & Raj, M., A-one Fertilizers & 62 & 0.78481 & 95 \\
\hline 2. & Meka, B., SECURE & 4 & 0.11765 & 11 \\
\hline 3. & $\begin{array}{l}\text { Kasi, N., Ramana } \\
\text { Fertilizers }\end{array}$ & 2 & 0.5 & 3 \\
\hline 4. & $\begin{array}{l}\text { Chiruvella, P., Victory } \\
\text { Pesticides }\end{array}$ & 1 & 0.33333 & 2 \\
\hline 5. & Rao, S., Simbha Fertilisers & 1 & 0.33333 & 2 \\
\hline 6. & $\begin{array}{l}\text { Alluri, T., Laxman } \\
\text { Fertilisers }\end{array}$ & 1 & 1 & 1 \\
\hline
\end{tabular}

\subsection{Social embeddedness of knowledge flows in the community of agriculturalists}

If all actors from outside the village are removed from the networks, the data are reduced to Punukula residents (farmers, farm labourers, shopkeepers - 211 nodes instead of the present 283). The 'experts' such as the all-in-one dealers and NGO representatives are no longer part of the networks (the only NGO representative who lives in Punukula is also not included). We arrive at a community of agricultural practitioners with $90 \%$ of network-members engaged in agriculture as their main source of livelihood. As noted earlier, this community has a dense network of social connections but relatively few farmer-to-farmer knowledge flows. In this section, I test the degree to which the latter are embedded in the former.

In at least two literature streams, relevant to the present study on farmers, a dense network of social ties is believed to act as foundation for interactive learning in a community. These literatures focus on communities of practice (see for example, Lave and Wenger 1991; Brown and Duguid 1991; Amin and Cohendet 2004); farmer field schools and farmer-centred participatory technology development approaches (e.g. Sherwood and Larrea 2001; Uphoff 2002). These studies emphasize that social ties in a community permit frequent face-to-face interactions that are essential for the transfer of tacit knowledge. The dense social network of the community acts as the 'soft architecture' of knowledge sharing and learning (Amin and Cohendet 2004): this soft architecture includes community cohesion, reciprocity, loyalty, belonging to a group, trust, shared practices and goals. These 'soft-architectural' features were present in the agrarian community of the village studied here. In the following, I analyse whether these features provide a strong foundation for knowledge sharing in the village.

As noted earlier, most knowledge flows take place between farmers and people from outside the village (such as the pesticide dealers and NGO representatives). After these outsiders are removed, the number of links in the 'reduced' knowledge network 
is only 109, as compared to 330 in the 'undivided' network. Thus, the density of the knowledge network reduces from 0.0041 to 0.0025 .

The network level values of social embeddedness of knowledge links in the village are listed in Table 9.9. Results are presented for embeddedness of knowledge flows in both direct and indirect social ties (the total number of social ties of the two types is 493 and 1796 respectively). As shown in the Table, 23.85\% and 39.45\% of knowledge flows in Punukula are coincident with corresponding direct and indirect social ties respectively. This level of proportional embeddedness for the reduced networks is substantially greater than that for the 'undivided' networks (where only $9.7 \%$ and $19.7 \%$ of the knowledge network was embedded in direct and indirect social ties respectively, see Table 9.1). ${ }^{42}$ However, the QAP correlation coefficients between the reduced knowledge and social networks are only marginally greater than corresponding values for the 'undivided' networks $(0.073$ vs. 0.047 for correlation with the social network of direct ties; and 0.053 vs. 0.044 for indirect social ties, $\mathrm{p}<0.001$ for all these results). Thus the structures of the knowledge and social networks of the agricultural community possess very little similarity to each other. Direct ties of the social network used by farmers to exchange knowledge are highlighted in Figure 9.4 using a map of the social network in Punukula.

Embeddedness of knowledge flows in the village may also be measured for a social network made up of both direct and indirect ties. In other words, one can compute the embeddedness of knowledge flows in direct friendship/kinship relations and indirect friends-of-friends ties simultaneously. The values of embeddedness, and correlation of knowledge network with a union of direct and indirect social ties are listed in the last row of Table 9.9. Here, $44 \%$ of the total 109 knowledge flows within the village take place among direct and indirect social relations. As compared to the results for the undivided networks, this result is more in accordance with what the literature would expect for knowledge sharing in a tight-knit agrarian community. However, the correlation coefficient remains poor $(0.063, \mathrm{p}<0.001)$. That is, the structure of the sparse knowledge network is significantly different from the dense social network composed of direct and indirect ties.

Table 9.9 Embeddedness and social capital for knowledge in Punukula only

\begin{tabular}{|l|l|l|l|l|}
\hline Path length of social ties & $\begin{array}{l}\text { Density of } \\
\text { the social } \\
\text { network }\end{array}$ & $\begin{array}{l}\text { Number of } \\
\text { embedded } \\
\text { knowledge } \\
\text { flows }\end{array}$ & $\begin{array}{l}\text { Embed- } \\
\text { dedness }\end{array}$ & $\begin{array}{l}\text { QAP } \\
\text { Correlation }\end{array}$ \\
\hline $\begin{array}{l}\text { Direct social ties (close kin } \\
\text { and friends) }\end{array}$ & 0.0111 & 26 & 0.2385 & $0.073^{*}$ \\
\hline
\end{tabular}

42 The proportion of total knowledge flows embedded in the 'reduced' social network is quite low: only $8.4 \%$ of the total 309 knowledge flows are embedded in direct social ties within the village and $13.9 \%$ in indirect ties. 


\begin{tabular}{|l|l|l|l|l|}
\hline $\begin{array}{l}\text { Indirect ties (friends of } \\
\text { friends) }\end{array}$ & 0.0405 & 43 & 0.3945 & $0.057^{*}$ \\
\hline $\begin{array}{l}\text { Both direct and indirect ties } \\
\text { friends/kin and friends of } \\
\text { friends) }\end{array}$ & 0.0429 & 48 & 0.4404 & $0.063^{*}$ \\
\hline
\end{tabular}

${ }^{*} \mathrm{p}<0.001$

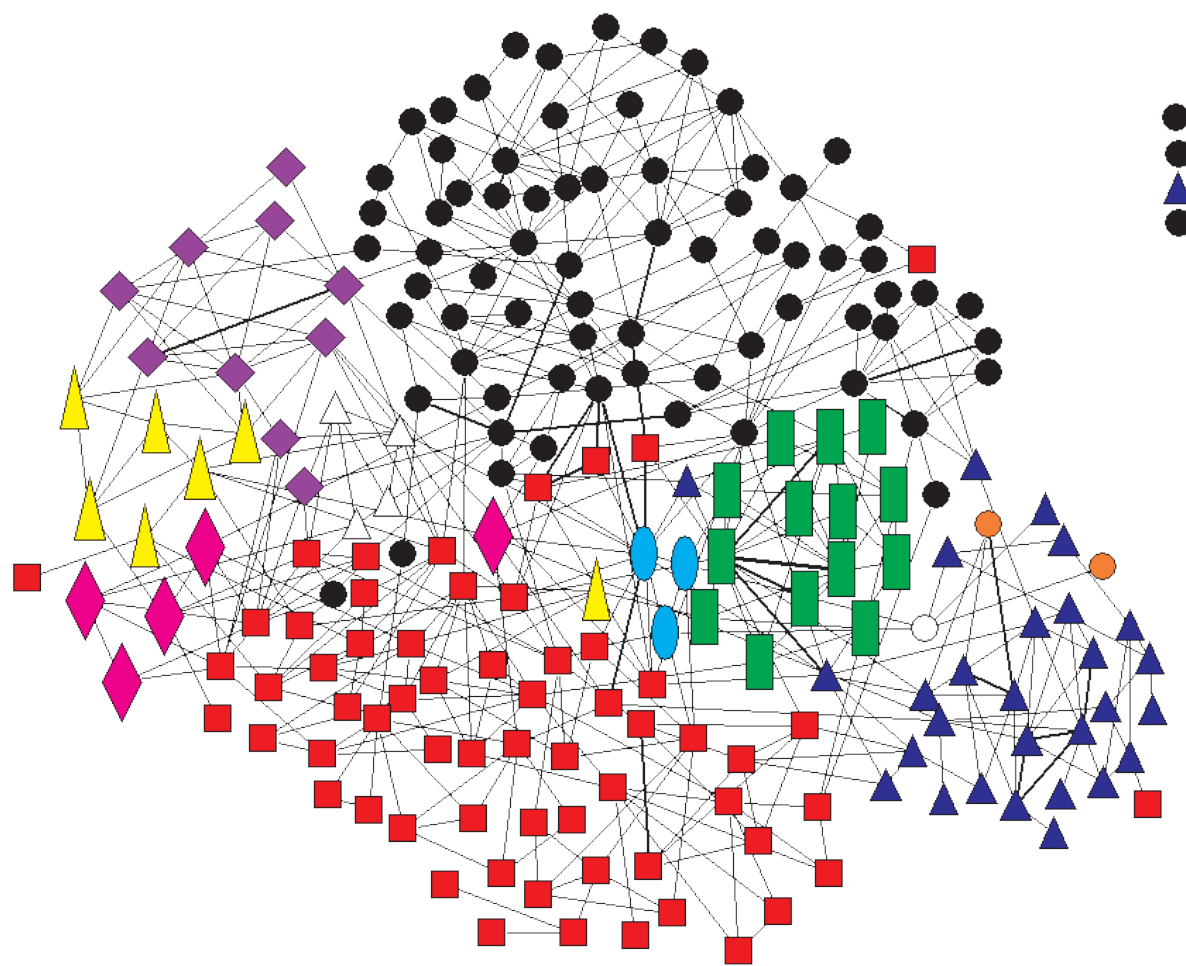

Figure 9.4 Knowledge flows embedded in the social network of the village community only (social ties which transfer knowledge are thicker). Nodes of different shapes and sizes represent people from different sub-castes (see Table 8.1 for details).

Results for the QAP regression on the 'reduced' social networks are shown in Table 9.10. In accordance with low correlation values, I get low values for the $\beta$ coefficient in the regressions for all three social networks. Of the three, the best predictability of the dependent variable (the knowledge network) is given by the social network made up of direct links. But even this $\beta$ coefficient is quite low and the regression fit very poor $\left(\mathrm{R}^{2}=0.005\right)$. Thus, having a dense social network does not produce a correspondingly dense or effective transfer of knowledge in the community. Few farmers share knowledge with their friends and kin. 
Table 9.10 Regression of the knowledge network on social network of the village

\begin{tabular}{|l|l|l|l|}
\hline Model & Direct social ties & Indirect social ties & $\begin{array}{l}\text { Direct and Indirect } \\
\text { social ties }\end{array}$ \\
\hline$\beta$ & $0.024^{*}$ & $0.010^{*}$ & $0.011^{*}$ \\
\hline Intercept & 0.0019 & 0.0016 & 0.0015 \\
\hline $\mathrm{R}^{2}$ & 0.005 & 0.003 & 0.004 \\
\hline
\end{tabular}

${ }^{*} \mathrm{p}<0.001$

For embeddedness in direct social ties within the village at the individual level, the pattern of results is broadly similar to those for the 'undivided' networks shown in Table 9.3 and 9.4. The main difference from the 'undivided' results is that the outsiders are missing from the lists now. So, only 21 (20) people out of the 211 Punukula residents have at least one incoming (outgoing) knowledge link that coincides with a social tie, as compared to 26 (24) out of the 283 members of the undivided networks. However, the general pattern of embeddedness values for the Punukula farmers in the reduced networks is largely the same as the results shown in Tables 9.3 and 9.4: this is because few farmers had any social ties with the NGO representatives and pesticide dealers from outside the village. ${ }^{43}$

The results on embeddedness presented in the foregoing show that the dense social network acts as a partial foundation for knowledge sharing and interactive learning in this community of agricultural practitioners. However, this partial foundation does not provide adequate social capital (for knowledge) to support interactive learning within the community. This is largely due to the fact that only a small share of the total knowledge flows in the local innovation system are embedded in the community (26 out of a total of 330 knowledge flows).

\subsection{Discussion}

In this chapter, I have shown that the degree of embeddedness of the knowledge flows of Punukula in its social ties is very weak: less than $8 \%$ of total knowledge flows

43 For purposes of comparison with the undivided networks, the values of embeddedness of individuals in the reduced network are listed in Appendix D of this thesis. Individual level values for embeddedness in indirect social ties in the village are also given in the same Appendix. One noteworthy difference from the undivided results is that more people in the reduced networks have the maximum possible degree of embeddedness: 15 for incoming and 11 for outgoing as compared to 8 and 10 for the same set of links in the undivided networks. This is due to the removal of the experts, along with their incoming and outgoing links from the networks. After this removal, the number of total knowledge links of the villagers drastically reduces (this number is in the denominator of the equation used to compute embeddedness). This difference between the villageonly and undivided networks is also reflected in the network level results for proportional embeddedness discussed above. 
(26 out of 330) are embedded in direct social relations and 13\% in indirect ones. As a result, the availability of social capital for knowledge in the village is very limited. In addition, little similarity is observed between the structures of the social and knowledge networks i.e., knowledge transfer over the dense social networks is not widespread and uniform but dispersed and sparse. Why aren't the knowledge flows embedded in the rich social network of the village with its strong ties that act as vehicles of trust, loyalty, reciprocity, and other manifestations of social cohesion? Below I attempt to answer this question based on my understanding of the situation in the village.

Let me begin with an attempt to address the limited social embeddedness of knowledge flows found in the community of agricultural practitioners studied in the previous section. First, successful learning communities possess routines that root useful knowledge in daily practices and habits (Brown and Duguid 1991; Amin and Cohendet 2004). Knowledge related to pest control, both for NPM and pesticides, was not ingrained in the daily practices of the farmers. As evidence from the knowledge network shows, most farmers depend on 'experts' such as the NGO representatives and pesticide dealers for accessing useful knowledge. ${ }^{44}$ This dependence did not allow a culture of local learning to flourish in the agrarian community of the village. ${ }^{45}$ Furthermore, the initial communities of practice literature studied communities where learning was a purposive activity (see for example, Lave and Wenger 1991). A primary aim of those communities constituted by teachers and their apprentices was transfer of knowledge and collective learning, in contrast, learning was not a purposive activity in the agrarian community of the village studied.

Second, the caste-based hierarchy in the village, albeit weak, violates the horizontal structure of social relations considered essential for a successful knowledge-sharing community. As shown in Figure 9.4, few people act as 'knowledge brokers' who connect the ten sub-caste based communities in the village (only 8 inter-caste social ties transfer knowledge). Thus, little bridging social capital for knowledge existed in the community. Third, due to the small size of the village community, the diversity of skills available in the village was small. Variety in skills and knowledge fosters knowledge

${ }^{44}$ This dependence on the experts may also be due to the nature of the two pest-control technologies in use. NPM was a relatively new technology in the village, so the farmers initially have to ask an expert when faced with a problem. Pesticides must be bought from the dealer who as a seller knows his product better than his customers.

45 The roots of this absence of a local culture of learning may be found in the history of the green revolution in Andhra Pradesh. Such a history is out of the scope of the present study but provides an interesting avenue for future research. Developing a new local culture of learning with the NPM intervention did not apparently succeed in the village: it simply ended up replacing the pesticide dealers with NGO representatives as central sources of knowledge for the NPM farmers. It is possible that building a local culture of learning takes longer than the 4-5 years (the NPM project started in the village in 2001 and I collected my data in the beginning of 2006). However, this possibility seemed bleak in Punukula as only a small number of farmers in the village continued to use NPM into 2005 (see Chapter 6, particularly Table 6.1). 
sharing and innovation by allowing learning to take place along different paths suitable to individual needs and problems.

Similarly, the degree of embeddedness in social ties and social capital for knowledge in the local innovation system was found to be low. ${ }^{46}$ Most knowledge flows take place between 'experts' and farmers who are generally not tied to each other in the social domain. This lack of linking social capital for knowledge is considered a major factor in the failure of development projects involving new knowledge or institutions (Szreter 2002). One reason for the low embeddedness of knowledge flows may be the expertise-based hierarchical relations between knowledge sources (NGO representatives, pesticide dealers, and others including government officers) and farmers. Almost all NGO representatives (and pesticide dealers) considered themselves as possessors of superior knowledge than the farmers. There was very limited linking of the 'expert' knowledge of the NGO representatives with the 'practitioner' knowledge of the farmers. Effects of this expertise-based hierarchy were not only observed in the technological arena but also filtered into the social relations among NGO representatives and the farmers. Barring a few 'progressive' farmers, there was a systematic lack of respect for most farmers from the NGO representatives. Social ties bridging the vertical divide between the NGO and the farmers were largely absent, creating little 'social space' for linking social capital for knowledge to flourish. ${ }^{4}$

Vertical ties in the business domain were, on the other hand, more important vehicles for knowledge transfer. A total of 71 links $(22 \%)$ of all knowledge links are embedded in the business network, as compared to 32 knowledge flows in direct social ties. Conversely, knowledge flows through a high percentage of $59 \%$ of the links in the business network as compared to only $6 \%$ of the social ties. The structure of the knowledge network possesses stronger resemblance to that of the business network for pest control (a correlation value of 0.351). Therefore, the business network of direct supplier-buyer relations acts as an effective vehicle for the exchange of relevant knowledge: these 'user-producer' interactions are a fundamental feature of an

\footnotetext{
46 Note that a very high degree of embeddedness and social capital may not necessarily yield better learning performance in a community or a local innovation system. The highest degree of social embeddedness in a community, for example, implies that all members of this community only share knowledge with their close friends and relatives. This small group of friends and relative, however, may not be a repository of new knowledge (and information) that is essential for innovation (or employment). Or, if the group is cliquish (the most cliquish would be a caveman graph), new knowledge even if present in the group may not leave it. Further, the kin of successful people may be a burden on them through unwanted requests of jobs and loans (Geertz 1963). Evans (1995; 1996) recommends that a sufficient degree of embeddedness must be complemented with an equal amount of 'autonomy' for dynamism in development.

${ }^{47}$ The 'progressive' farmers were those who fervently adopted the new technologies and practices promoted by the NGO representatives. Critical thinking, dissonance, or even experimentation on part of the farmers was not appreciated by the NGO or the pesticide dealers. Competitive critical thinking is often considered essential for learning and innovation in communities (Amin and Cohendet 2004).
} 
innovation system, which were recognized in early important works by Lundvall (1985; 1988).

\subsection{Conclusions}

In this chapter, I have attempted to refine the concepts of embeddedness and social capital by exploring their structural roots in networks of strong social ties. I argued that an impressive set of studies has focussed on the network structure component of embeddedness and social capital. In contrast, accompanying work on tie content component of embeddedness has been fragmentary. I argued that in order to derive useful results from embeddedness in networks, one must be precise about the content of ties that make up the networks. This specification of tie content is important for knowing the exact nature of transactions that are embedded in different types of ongoing social relations (in clarifying what exactly is embedded in what?). In other words, one must specify the content of the (economic) transactions that are embedded, and the content of the 'social' ties that embed them. Concurring with Podolny and Baron (1997), I argued that well-accepted conclusions on the effect of embeddedness on performance, through structural holes and closure, may diverge depending on the content of ties. ${ }^{48}$

I presented results on the degree of embeddedness of knowledge flows in social networks, of strong kinship/friendship ties, and business networks forming a local innovation system. The results show that the embeddedness and social capital of knowledge flows in social ties are low in this innovation system (less than $10 \%$ and $20 \%$ of knowledge links are embedded in direct and indirect social ties respectively). The correlation between the knowledge and social networks was also found to be poor. The knowledge network is more highly correlated with the business network of pest control: a correlation value of 0.351 is found between the knowledge and the business network. A total of 71 links $(22 \%)$ of all knowledge links are embedded in this business network (in reverse embeddedness terms, this translates into $59 \%$ of all links in the business network). Therefore, user-producer interactions in the business network of direct supplier-buyer relations are critical for transfer of knowledge and learning in this innovation system.

A majority of the links in the knowledge network are however not embedded in business or social relationships. These 'missing' knowledge links take place largely between NGO representatives and farmers who are not connected to each other in the social or the business sphere. This overwhelming prevalence of unembedded knowledge links is a striking result which underlines the lack of social capital for knowledge in the village. This lack of social capital for knowledge, both of the bridging

48 Although I did not explore the significance of closure vs. structural holes in the knowledge, business and social networks of Punukula. This was largely due to my focus on identifying coreperiphery structures in the three networks (chapters 6,7 , and 8) and the fact that two of the three networks have directed links. Investigation of the (variable) influence of closure and structural holes in the three networks may be a fruitful avenue for future research with Punukula data. 
and linking varieties, may be one of the reasons behind the failure of the Punukula NPM project in building innovation capacity among the farmers, as discussed in Chapter 6.

If the networks are reduced to include Punukula residents only, the percentage of knowledge flows embedded in strong social ties increases to approx. $24 \%$ and $40 \%$ in direct and indirect ties respectively. Thus, social ties within the agricultural community provide some foundation for knowledge sharing. However, the number of links in the reduced knowledge network is dramatically lower than the undivided network (109 as compared to 330). The correlation between the structures of the social and knowledge networks is still poor. Therefore, despite the presence of a soft architecture provided by the dense social network of the community, knowledge flows remain scattered and embeddedness low.

At the individual level, results on the embeddedness of incoming and outgoing knowledge links measure the social capital for knowledge access and from knowledge delivery. Fewer than $10 \%$ of people in the networks ('undivided' and 'reduced') have a non-zero value of social capital for knowledge access. Of the few people with nonzero social capital values, many are prominent individuals in Punukula including the village council president, community headmen and their close kin.

Social capital from knowledge delivery is more akin to power or dominance an individual possesses due to her position in the social and knowledge networks. The access and delivery types of social capital are however interrelated: $40 \%$ of the people with non-zero social capital for knowledge delivery also have non-zero social capital for knowledge access. A few community headmen in the village and the main pesticide supplier to the village once again hold the highest social capital values for knowledge delivery. The latter's social capital for knowledge delivery to friends of friends (indirect ties in the social network) is clearly the highest in the networks.

Finally, individual level embeddedness of incoming knowledge links in business ties is greater than that in the social network. Sixty-seven people have incoming knowledge ties coincident with their incoming business ties in the network for procuring pest control. The number of people with embedded outgoing knowledge links in corresponding business ties are however fewer than those in the social network. The list of people with embedded outgoing links here is clearly dominated by the main pesticide supplier who possesses approximately $90 \%$ of these links.

To conclude, the literature on communities of practitioners and local innovation systems highlight the importance of social cohesion, in terms of dense social networks, for knowledge sharing and innovation. Some go as far as to claim that dense social networks in which the ties transmit trust are necessary for learning and innovation. Here learning and innovation are considered as interactive activities involving multiple actors for which social networks provide the cementing glue. However, contrary to what one would expect based on this literature, I find that the dense social network of the village provides a very limited basis for its knowledge flows, particularly when all the knowledge flows to and from the village are considered. When the knowledge 
flows are reduced to those taking place within the village only, embeddedness of these fewer knowledge flows in the social network increases. Thus I find that a significantly greater degree of knowledge flows are socially embedded in the community of agricultural practitioners than in the local innovation system. This result is in line with studies focussed on knowledge-sharing within communities, which generally find a relatively high degree of social embeddedness of the knowledge flows. But by ignoring knowledge transfer from external sources, which in the present case form approximately two-thirds of the total knowledge flows, these studies produce results that are biased (toward higher degrees of social embeddedness).

I find that user-producer interactions along direct buyer-supplier relationships in the business network provide a fertile substratum for the transfer of relevant knowledge. Thus, the emphasis on social cohesion and social capital in recent literature on knowledge sharing and learning must be re-evaluated using a network-based methodology as the one presented here. Such a re-evaluation may lead to reinstatement of user-producer interactions as the primary element in building (local) innovation systems. 


\section{IO. Summary and conclusions}

This dissertation was divided into a theoretical part I and an empirical part II. The theoretical part presented a novel way of looking at positive feedbacks in technological evolution. New insights were provided on the structure of the positive feedback loops and their strength vis-à-vis each other. The latter has important implications for cases in socio-technical systems where a technology succession is imminent. In part II of the thesis, the theoretical results were applied to an NGO-led development intervention in one Indian village. The empirical analyses in part II have important implications for the process of socio-economic development. By focussing on an innovative development intervention in one village, I uncovered critical social structural factors that drive the process of rural development more generally. Some concluding implications of the observations and results from the empirical chapters are discussed later. Below I start with a brief chapter-by-chapter summary of the dissertation.

\section{IO.I Summary of the results}

A primary aim of the theoretical research was to investigate the neglected topic of technological interdependence, and to analyse how the structure of underlying positive feedbacks between different interdependent technologies influences a process of technology succession. Review of two streams of literature provided an initial impetus for this work. First, I reviewed some models of technological evolution such as the classic technology life cycle model of Abernathy and Utterback (1975); the paradigmtrajectories model of Dosi (1982); and the guidepost-avenues model of Sahal (1985). Second, useful complementary insights were gained from studies on the socioeconomic history of different technological systems including the American system of manufactures (Hounshell 1984); the gradual construction of electrical power systems in the USA (Hughes 1983; David 1990); and the post-war development of a new textile technological system around synthetic fibres (Hounshell and Smith 1988a; 1988b). Furthermore, the valuable historical analyses of Usher (1954) and Rosenberg (1976; 1982) provided concepts for building bridges between history and theory.

The part I of the dissertation was then an analysis of technological evolution with particular emphasis on technology succession in systems replete with complementarities and interdependencies, or in a dynamic framework, feedbacks between different system components. A literature review on the mechanisms of technological interdependence, and the structure of positive feedback loops, formed the focus of chapter 2 of the thesis. This review-based discussion formed the historical and theoretical background for the model of technological evolution developed in chapter 3.

Economists have studied the role of positive feedbacks among interacting technologies in analyses of technology competition (see for example, David 1985; Arthur 1989; and Cowan 1991). Related work on technology succession was performed by economists interested in understanding the nature of technological lock- 
in, for example, due to network effects, installed base effects and standardization (see Cowan and Hultén 1996; Unruh 2000; Gerlagh and Hofkes 2002). A technological succession or a transition then implies 'escaping a lock-in' (Cowan and Hultén 1996). Interdependence between two substitutable technologies in a process of succession formed the first mechanism of technological interdependence reviewed in Chapter 2. This interdependence is visible in historical cases of technological succession such as those studied by Musson (1976); and Hounshell and Smith (1988a; 1988b): new technologies were commonly modeled on the old technologies they were meant to replace. More generally, however, the new technology had to fit an existing system that was designed and built around the old technology. For example, the new synthetic fibres were woven on machines originally developed for natural fibres (Hounshell and Smith 1988a).

The systemic aspect of technologies forming complementary components of larger complex product and technological systems was studied by historians such as Rosenberg (1976; 1982); Hughes (1983) and Hounshell (1984). The knowledge types underlying a complex product or technological system might originate in diverse fields such as computer software and mechanical design. This horizontal interdependence was the second mechanism identified in chapter 2. A third mechanism of technological interdependence concerned technologies with a large number of horizontal and vertical interdependencies. Economists have termed these technologies generic or generalpurpose technologies, GPTs (see for example, David 1990; Bresnahan and Tratjenberg, 1995; and Lipsey et al. 1998). GPTs, such as the transportation and communication technologies, provide the general conditions for production in an economy and possess a large number of forward (output-side) linkages. As a result, effects from improvements in the efficiency of these generic technologies are felt throughout an economy.

The last mechanism of technological interdependence identified concerned the vertical relationships between technologies connected through production chains. These linkages, in the context of economic exchange as buyer-seller links in commodity chains, were studied by world-system analysts such as Wallerstein (1983). These user-producer linkages also form the cornerstone of 'learning by interacting' in the innovation systems framework developed by Lundvall (1992) and Nelson (1993).

The temporal aspects of technological interdependence were reviewed next in Chapter 2 and the different mechanisms of interdependence were inserted into an explicitly dynamic framework. Review of the temporal aspects was structured by a punctuated-equilibrium view of technological evolution, which makes a clear distinction between the gradual phases and the discontinuities in evolution. During the former, individual technologies (might) improve in efficiency while retaining their set of interdependent technologies. This set of horizontal and vertical interdependencies is 
disrupted by a discontinuity. ${ }^{1}$ In the gradual phase, improvements in one technology send positive feedbacks to other interdependent ones. I argued that the structure of these feedbacks influenced the strength of an incumbent technological system in the face of a discontinuity. A self-reinforcing circular process of feedbacks for example provided some inertia to an existing set of (locked-in) technologies.

Building on the literature review, a simulation model of technological evolution in a system of technologies or their underlying knowledge types was developed in Chapter 3. Components of technological systems were treated as core or periphery, allowing a skewed distribution of innovative dynamism among system components. Some self-sustaining components formed a system's core, providing innovative dynamism to the system as a whole. The model allowed an investigation of the impact of different core structures on system evolution using the theoretical construct of corestrength. This strength is a function of the structure of positive feedbacks among the core components of a system. In particular, a circular process or a cycle in graph theoretic terms is the minimum condition for the formation of a core. The density of internal feedback loops within the circular process determines the strength of a core. Finally, I presented results from simulation experiments where a system built around an existing core is threatened by the exogenous arrival of a competing circular process (\$3.4). If the new competing structure was stronger than the existing core, a technology succession took place. Subsequently, a new system is gradually built around the new dominant core.

Results from part I of the thesis on the circular process of positive feedbacks were used in the empirical part II of the thesis to analyse a rural community's experience with technology succession. The gamut of the positive feedbacks however was extended in part II to include the embeddedness of the community's techno-economic activities in its social networks. The community studied, a village in the Andhra Pradesh province of India, underwent a transition to non-pesticidal management of crops (NPM) from using pesticides. NPM was introduced to the village by a local grassroots organization working in cooperation with a 'knowledge NGO' specializing in sustainable agricultural practices. The NPM project in the village started in 2001 when a small number of farmers adopted NPM. The number of adopters rose gradually and, by 2004, the village had gained fame across India for stopping the use of pesticides completely. This transition to the low-cost NPM occurred with no loss in crop yields (Chapter 4 on details of the media and NGO reports). Furthermore, numerous media reports claimed that farmers had freed themselves of indebtedness because of the profitable pesticide-free agriculture practised in the village. ${ }^{2}$ This

${ }^{1}$ Furthermore, what is termed a discontinuity or a radical innovation ex-post is generally a gradual process of assembling a number of small incremental inventions, often in disparate interdependent technologies (see Chapter 3).

2 Most farmers in the village cultivated cotton which is the most pesticide-intensive crop in India. According to some estimates, about $40 \%$ of all pesticides in India are sprayed to protect the cotton crop. 
seemed to have revived the hopes of farmers all over the country suffering from an agrarian crisis caused by high costs of cultivation and falling crop prices. ${ }^{3}$

During the fieldwork in India from August 2005 to April 2006, I collected new data on the networks of this community. These network maps included all inhabitants of the village and their contacts for business and knowledge needs from outside the village (methodology of data collection discussed in Chapter 5). I focused on three different types of networks among the same group of people. The first was a map of the knowledge flows for pest control which included the two technologies: pesticides (old) and NPM (new). The second network mapped the business transactions of the farmers to access credit, sell crops, and purchase farm-inputs (seeds, fertilizers and pesticides). The last network mapped the social relations of close friendship and kinship ties. In addition to the network maps, I collected data on the extent of NPM adoption in the village, the farmers' ability to experiment with NPM and the sustainability of these two activities.

The part I results were then used to analyse the knowledge network structure and explain the nature of the farmers' innovation capacity (Chapter 6). Building the farmers' capacity building was a central aim of the NPM project of the NGOs. I found that the NPM innovation capacity among the farmers in was very limited: only 3 out of a total 155 farmers modified NPM methods through on-farm experimentation. In addition, less than half of the farming households used NPM in a season. A third of these NPM farmers reverted to pesticides on one or more crops during the season of the fieldwork (Kharif 2005-6). Thus, pesticides continued to be the most popular pestcontrol technology in the village and the transition to NPM or no-pesticides was never complete. The structure of the knowledge network of the village, viewed through the lens of circular flows and core-strength, explained the lack of farmers' innovation capacity (and limited NPM adoption) reasonably well. I found that, with the exception of a two-way flow between two brothers, all circular flows of knowledge involved a pesticide dealer or a NGO representative. The dominant core in the network was constituted by the main pesticide supplier to the village and three farmers. The strongest competing circular process (focused on NPM) was formed by four NGO representatives and two farmers. However, the dominant core structured around the main pesticide supplier survived all threats posed by competing circular processes created by the NPM project in the village. The pesticide supplier remained the main

\footnotetext{
${ }^{3}$ For example, in the period 1995-2001, global prices had fallen by about $40 \%$ for wheat, $50 \%$ for cotton and 30\% for soyabean. According to the Research Foundation for Science, Technology and Ecology, Indian smallholder farmers were losing $\$ 26$ billion a year due to the falling prices (Shiva 2004). On the input side during the same period, the costs of sourcing water, seeds, fertilizers, and pesticides had by contrast increased due to scaling down of subsidies by the government and rising petroleum prices. The resulting lack of profitability of farmers sealed them in a debt trap. Places where cultivation of input-intensive commercial crops such as cotton was prevalent were the worst affected. According to a recent National Sample Survey report, $82 \%$ of the farmers in Andhra Pradesh were indebted. The same percentage for the whole of India stood at $48.6 \%$ (Chandrasekhar and Ghosh 2005).
} 
pest-control knowledge provider with the highest out-degree centrality in the network. His direct and indirect connections allowed him to reach 119 out of the total 155 farmers in the village as compared to only 67 farmers reached by the best-connected NGO representative.

In the business network of the village, the dominance of the main pesticide dealer came to the fore more prominently (Chapter 7). The dealer buttressed his power in the knowledge domain through his control over the village in the business domain. He not only sold all farm-inputs to most farmers but also bought their crops. As a result, 161 of the 173 circular processes in the business network of all transactions involved this main pesticide dealer. The dominant core in the network was constituted by him, another pesticide dealer, and two farmers from the village (most other circular processes were of size 2 formed by the main dealer and a farmer who bought farminputs and sold his crop to the dealer).

I used the social network structure of the village to explain the socio-political activities surrounding NPM, particularly the unified representation of the pesticide-free status to the outside world. The premise of my argument was that the NGOs required support from the villagers to sustain a unified representation of the no-pesticides success. This support was enabled through collective action by some local elites in the village. The aim in Chapter 8 was then to understand the social-structural roots of this collective action using the theoretical constructs of circular process and core-periphery in the network. In a social network with its symmetric links, a circular process exists between any two nodes. To circumvent this problem, I used positional measures of influence and brokerage in social networks to identify a small group of local elites. The (bi-directional) circular process formed by these local elites was then taken to form the core of the social network. This core group of people acted as the 'face' of the village to the visiting journalists, controlling the representation of NPM events in the village. Most media reports quote one of the members of the core on the success of NPM.

Half of the members of the social-network core were heads of their respective sub-caste based communities in the village and the relationships among them represented important bridges that brought the village together. This 'bridging' social capital of the core group, coupled with their influence in their respective communities, allowed them to project the NPM success uncontested. Furthermore, one-half of the core members acted as the village-level resource persons of the grassroots NGO which initiated the NPM project. These connections with development actors outside the village gave the core group its necessary 'linking' social capital. An effective combination of the bridging and linking social capital allowed the core group to represent the village in all fora and suppress alternate versions of the NPM experience for a few years.

In chapter 9, the focus shifted from positive feedbacks within single networks to an investigation of the interrelatedness between the different networks of the village. Recent studies in innovation systems and communities of practice have argued that dense social networks enable the interactive processes of innovation and learning. 
These studies have claimed that sharing of knowledge, in particular of the tacit variety, requires extensive face-to-face contact which is facilitated by close 'social' (friendship, kinship) ties. So the main aim of Chapter 9 was to study the embeddedness of the network of knowledge flows in a network of social ties among the same group of people.

The chapter presented a critical overview of the literature on social embeddedness, in particular structural embeddedness. I found that while the network structural aspects of embeddedness were widely analysed, parallel analysis of tie content was rare. This neglect of tie-content could produce erroneous results (see Podolny and Baron 1997; Rodan and Galunic 2004). Content of ties occupied central place in my work through the focus on the embeddedness of knowledge links in 'social' ties. To determine this embeddedness, I defined a simple measure of the degree of embeddedness of one network in another, and used the Quadratic Assignment Procedure for correlating the two networks and performing regressions of one network on another. These measures were computed at two levels: first for the 'complete' networks of the village which included the NGO representatives and pesticide dealers (referred to as the local innovation system); and second for 'reduced' networks in the village which was simply the community of agricultural practitioners. For the local innovation system, the results showed that the dense social network of friendship and kinship ties of the village acted as a weak foundation for knowledge sharing. Less than $10 \%$ of the knowledge flows took place among people tied to each other as friends or kin. A substantially larger percentage $(22 \%)$ of the knowledge flows took place among direct business contacts, such as those between a pesticide dealer and a farmer. Furthermore, the knowledge network was much better correlated with the business network (a correlation value of 0.35 ) than with the social network (0.05). Therefore, user-producer interactions among direct business relations provided a much better foundation for knowledge sharing than close 'social' ties in the local innovation system.

In the community of agriculturalists in the village, $24 \%$ of the knowledge flows are embedded in direct social ties and a total of $44 \%$ of knowledge flows take place among people directly and indirectly connected in the 'social' sphere. That is, almost half the knowledge flows in the village take place among friends/kin and friends of friends. Thus, I found greater evidence for the embeddedness of knowledge sharing in social relations within the community than in the larger innovation system. However, only a third of the total number of knowledge flows take place within the community. The majority of the knowledge flows involve 'experts', such as the pesticide dealers or NGO representatives, from outside the village.

\section{I0.2 Implications for rural development interventions}

The research presented in this dissertation has three implications for the understanding rural development interventions. First, success in (technological) development interventions is often not the successful implementation of development 
policies or project plans (and meeting the planned objectives), but the control of representation of important events in the project (cf. Mosse 2004; 2005). This was evidently the case in the village studied where the representation of NPM events in the media related to the complete stop on the use of pesticides was controlled by the involved NGOs and crucially, a set of important farmers from the village. As noted in Chapter 4, the control of the representation of NPM success by the involved NGOs was evidenced by their publications and media reports written by their staff (see Ramanjaneylu et al. 2004; 2005; Kuruganti 2004a; 2004b). In addition, other media reports on the no-pesticides and NPM success in the village relied on testimonies of a few farmers who together acted as the 'face' of the village. These farmers being familiar with the use of NPM methods and the farming conditions in the village were able to offer a convincing insiders' account of NPM success. In Chapter 8, I showed how this group of farmers belonging to different castes managed to present a unified representation of NPM success. I argued that this representation was facilitated by friendship connections among these farmers. The connections bridged the caste divides in the village as a whole because many of the farmers in this group happened to be influential heads of their respective caste-based communities. In addition, the formation of such a strong sub-network of community leaders also discouraged dissenting representations of the NPM events by other villagers. Eventually, dissenting opinions arose from within this group creating a split and two contrasting views on the success of NPM methods and the NGO-led development intervention more generally (\$8.5). Therefore, even though a coherent representation of success in the village was created and maintained for some time by the involved actors through the alignment of different interests, the representation was difficult to sustain indefinitely because of the difference in interests themselves (cf. Mosse 2005). One example of the difference in interests in the core group of influential farmers was the pull of friendship that some of them had with the main pesticide supplier to the village, which rivalled their association with the NGOs for NPM.

Second, the process and outcome of development interventions are driven by routines (existing habits and practices) of the actors involved, and not by project plans defined ex-ante (cf. Mosse 2004). The NGOs' predefined objective in the NPM project was to develop an innovative capacity among the farmers in the village to experiment for modifying existing NPM methods to suit their needs, and whenever possible invent new ones. The NGOs designed a set of plans and policies that would allow them to achieve the objective effectively. These plans included basing NPM on existing farmers' knowledge; extensive training of the farmers on all aspects of NPM; identifying 'progressive' farmers avidly interested in the NPM alternative and working closely with them; appointing 10-15 influential farmers as the NGO's resource persons in the village; forming a farmers' group (Raithu Mitra Group) which met regularly to facilitate decision-making and knowledge sharing; installing a mill to crush neem seeds in the village for preparation of the neem spray (an essential NPM component) and training two people to operate the mill; and stationing an NGO representative in the 
village for ready availability of NPM-related assistance to the farmers. These plans were surely implemented and enabled people with diverse interests to work together, but the process of implementation that drove the project outcomes was ridden with the involved actors' routine way of doing things. These routines for example included the top-down interactions between the NGO representatives and the farmers. In general, the NGO representatives showed a lack of respect for farmers' knowledge and practices. In the knowledge network, this knowledge hierarchy manifested itself as an almost-complete absence of two-way knowledge flows among farmers and NGO representatives. This 'difference' was also observed in the social network of the village where few relations of friendship and conviviality existed between farmers and NGO representatives (Chapters 8 and 9). Furthermore, the farmers considered 'progressive' by the NGOs were not those critical of received knowledge and practices but those who fervently adopted the advocated NPM practices. Thus, the progressive farmers, rather than being the critical experimenters, simply acted as sounding boards for the NGOs' 'expert' knowledge.

Equally important in this process were the routines of the farmers themselves. Before the NPM intervention, the farmers simply procured pesticides and sprayed them on the crop a few times every season. The NPM intervention did not bring about a change in these routines of the farmers - it simply replaced the pesticide supplier with the NGO representatives for the NPM-using farmers (Chapters 6 and 7). Pestcontrol related knowledge sharing and learning were not rooted in daily practices of the farmers (cf. Amin and Cohendet 2004). The existing routines of the farmers did not include on-farm experimentation and knowledge sharing: farmers habitually sourced problem-solving knowledge from the NGO representatives for NPM and from the pesticide dealers for pesticides. These routines limited the extent of learning and build-up of innovation capacity among the farmers.

My final point is that in addition to the actors' routines, a combination of bonding (within group), bridging (between groups) and linking (across vertical divides) social capital drives the outcomes of development interventions. Those parts of development interventions that work with, and allow people to maintain and strengthen their, existing social relations are the ones that 'succeed' (the example of media success in Chapter 8). ${ }^{4}$ Conversely, activities that are not embedded in social relations, or in other words lack the three types of social capital, have a smaller chance of succeeding sustainably (for example, the lack of social capital for knowledge and the failure of innovative capacity building in the village, Chapters 6 and 9). The latter is particularly true for cases where other forms of buttressing ties such as durable business or social relationships are not present. Thus, the actors' need and ability to maintain social

\footnotetext{
4 This need of actors at all levels to maintain social relations may also be viewed as a routine of the actors. Then it follows from the second point above that the development plans or policies that recognize this need for maintaining social relations have a greater chance of driving the practice of development projects.
} 
relations, along with adherence to everyday routines discussed in the second point above, drive development interventions.

The social and durable business relations and the everyday routines of the actors are in turn socially constructed. Institutions, both of the formal and informal variety, in a society determine how people relate to each other and practise their everyday lives. But how does this process of social construction of relations and everyday routines work? Or why do the networks mapped in Punukula look the way they do? Can new technologies and practices be embedded in the social relations and everyday practices of the beneficiaries of development? On the other hand, can or should development interventions really ask people to 'bend' their everyday practices to suit the means and ends of the intervention itself? Or are people themselves willing to bend their practices anyway - tactically to harness benefits from development? And finally, can development interventions be framed so they are not at odds with pre-existing social institutions? Answering these questions requires historical-institutional analyses of network formation and everyday routines of people. These types of analyses form interesting avenues for future research.

The analyses of part II of thesis, apart from providing interesting avenues and raising questions for future research, have shown that network-based studies can provide a new way to look at the practice of rural development. In addition, such analyses, by allowing one to anchor research into the structural territory of social relations and their resulting social capital effects, yield critical insights into the uses and abuses of the latter as an analytical construct in development studies. As I demonstrated in chapters 6-9, a large amount of the so-called social capital can leave vulnerable smallholder farmers out of the innovation-development nexus that was meant to liberate them from the clutches of oppressive institutions and their reinforcing technologies. 


\section{References}

Abernathy, W. 1978. The Productivity Dilemma: Roadblock to Innovation in the Automobile Industry. Baltimore, MD: Johns Hopkins University Press.

Abernathy, W., and K. Clark. 1985. Innovation: Mapping the Winds of Creative Destruction. Research Policy 14: 3-22.

Abernathy, W.J., and J.M. Utterback. 1978. Patterns of Industrial Innovation. Tech. Rev: 41-47.

Agrawal, A. 1995. Indigenous and Scientific Knowledge: Some Critical Comments. Indigenous Knowledge and Development Monitor 3 (3).

Agrawal, N. 2002. Indigenous Knowledges and the Politics of Classification. International Social Science Journal 173: 325-336.

Ahuja, G. 2000. Collaboration Networks, Structural Holes, and Innovation: A Longitudinal Study. Administrative Science Quarterly 45: 425-55.

Almeida, P., J. Song, and R.M. Grant. 2002. Are Firms Superior to Alliances and Markets? An Empirical Test of Cross-Border Knowledge Building. Organization Science 13 (2): 147-161.

Amin, A., and P. Cohendet. 2004. Architectures of Knowledge: Firms, Capabilities and Communities. Oxford: Oxford University Press.

Amin, S. 1982. Small Peasant Commodity Production and Rural Indebtedness: The Culture of Sugarcane in Eastern U.P., C. 1880-1920. In Subaltern Studies, edited by R. Guha. New Delhi: Oxford University Press.

Anderson, P., and M.L. Tushman. 1990. Technological Discontinuities and Dominant Designs: A Cyclical Model of Technological Change. Administrative Science Quarterly 35: 604-633.

Anheier, H.K., J. Gerhards, and F.P. Romo. 1995. Forms of Capital and Social Structure in Cultural Fields: Examining Bourdieu's Social Topography. The American Journal of Sociology 100 (4): 859-903.

Arthur, B. 1988. Competing Technologies: An Overview. In Technical Change and Economic Theory, edited by G. Dosi, C. Freeman, R. Nelson, G. Silverberg and L. Soete. London, UK: Pinter.

Arthur, B. 1989. Competing Technologies, Increasing Returns, and Lock-in by Historical Events. Economic Journal 99: 116-131.

Asheim, B.T., and L. Coenen. 2005. Knowledge Bases and Regional Innovation Systems: Comparing Nordic Clusters. Research Policy 34 (8): 1173-1190.

Asheim, B.T., and A. Isaksen. 2002. Regional Innovation Systems: The Integration of Local 'Sticky' and Global 'Ubiquitous' Knowledge. Journal of Technology Transfer 27: 77-86.

Astley, W.G. 1985. The Two Ecologies: Population and Community Perspectives on Organizational Evolution. Administrative Science Quarterly 30: 224-241.

Attwood, D.W. 1974. Patrons and Mobilizers: Political Entrepreneurs in an Agrarian State. Journal of Anthropological Research 30 (4): 225-241. 
Baldwin, R.E., and R. Forslid. 1999. The Core-Periphery Model and Endogenous Growth: Stabilising and De-Stabilising Integration NBER Working Papers 6899: National Bureau of Economic Research, Inc.

Barnett, W.P. 1990. The Organizational Ecology of a Technological System. Administrative Science Quarterly 35: 31-60.

Batagelj, V., and A. Mrvar. 2007. Pajek - Program for Large Network Analysis.

Baumol, W.J., and A.S. Blinder. 1985 Economics: Principles and Policy. 3rd Edition ed. San Diego: Harcourt Brace Jovanovich.

Bentley, J.W. 1994. Facts, Fantasies, and Failures of Farmer Participatory Research. Agriculture and Human Values 11 (2-3): 140-150.

Bian, Y. 1997. Bringing Strong Ties Back In: Indirect Ties, Network Bridges, and Job Searches in China. American Sociological Review 62: 366-385.

Biggs, S. 1990. A Multiple Source of Innovation Model of Agricultural Research and Technology Promotion. World Development 18 (11): 1481-99.

Bijker, W.E. 1995. Of Bicycles, Bakelites, and Bulbs: Toward a Theory of Sociotechnical Change. Cambridge, MA: MIT Press.

Block, F. 2001. 'Introduction' to the Great Transformation by Polanyi. Boston, MA: Beacon Press.

Bonacich, P. 1987. Power and Centrality: A Family of Measures. The American Journal of Sociology 92 (5): 1170-1182.

Borgatti, S.P., and M.G. Everett. 1999. Models of Core/Periphery Structures. Social Networks 21: 375-395.

Borgatti, S.P., M.G. Everett, and L.C. Freeman. 2002. Ucinet for Windows, Version 6.59: Software for Social Network Analysis. Harvard, MA: Analytic Technologies.

Bourdieu, P. 1986. The Forms of Capital. In Handbook of Theory and Research for the Sociology of Education, edited by J. Richardson. New York: Greenwood.

Breschi, S., F. Lissoni, and F. Malerba. 2003. Knowledge-Relatedness in Firm Technological Diversification. Research Policy 32: 69-87.

Breschi, S., and F. Malerba. 1997. Sectoral Systems of Innovation: Technological Regimes, Schumpeterian Dynamics and Spatial Boundaries. In Systems of Innovation: Technologies, Institutions and Organizations, edited by C. Edquist. London: Pinter Publishers.

Bresnahan, T., and M. Tratjenberg. 1995. General Purpose Technologies: 'Engines of Growth'. Journal of Econometrics 65: 83-108.

Brown, J., and P. Duguid. 1991. Organizational Learning and Communities of Practice: Toward a Unified View of Working, Learning, and Innovation. Organization Science 2 (1): 40-57.

Bruland, K. 1998. The Babcock \& Wilcox Company: Strategic Alliance, Technology Development, and Enterprise Control, Circa 1860-1900. In Family Firms to Corporate Capitalism, edited by O. B. Bruland. Oxford, UK: Clarendon Press. 
Brynjolfsson, E., and L. Hitt. 2000. Beyond Computation: Information Technology, Organizational Transformation, and Business Performance. Journal of Economic Perspectives 14 (4): 23-48.

Burt, R. 1983a. Corporate Profits and Cooptation: Networks of Market Constraints and Directorate Ties in the American Economy. New York: Academic Press.

Burt, R. 1983b. Distinguishing Relational Contents. In Applied Network Analysis, edited by R. Burt and M. Minor. Beverly Hills: Sage Publications.

Burt, R. 1992. Structural Holes: The Social Structure of Competition. Cambridge, MA.: Harvard University Press.

Burt, R. 2000. The Network Structure of Social Capital. In Research in Organizational Behaviour, edited by R. I. Sutton and B. M. Straw. Greenwich, CT.: JAI Press.

Burt, R., K.L. Lieben, and M.G. Fisher. 1980. Network Power Structures from Informant Perceptions. Hum. Organ. 39: 121-133.

Byerlee, D. 1998. The Search for a New Paradigm for the Development of National Agricultural Research Systems. World Development 26 (26).

Callon, M. 1987. Society in the Making: Study of Technology as a Tool for Sociological Analysis. In The Social Construction of Technological Systems, edited by W. E. Bijker, T. P. Hughes and T. Pinch. Cambridge, MA: MIT Press.

Carlson, W.B. 2000. Invention and Evolution: The Case of Edison's Sketches of the Telephone. In Technological Innovation as an Evolutionary Process, edited by J. Ziman. Cambridge, UK and New York: Cambridge University Press.

Chambers, R., A. Pacey, and L. Thrupp. 1989. Farmer First: Farmer Innovation and Agricultural Research. London, UK: Intermediate Technology Publications.

Chandrasekhar, C.P., and J. Ghosh. 2005. The Burden of Farmers' Debt. The Hindu Business Line, August 30.

Choudhury, S. 2004. Do It with Chillies. Outlook India, November 22.

Clark, K.B. 1985. The Interaction of Design Hierarchies and Market Concepts in Technological Evolution. Research Policy 14: 235-251.

Cohen, W.M., and D.A. Levinthal. 1990. Absorptive Capacity: A New Perspective on Learning and Innovation. Administrative Science Quarterly 35 (1): 128-152.

Cohn, B.S., and M. Marriott. 1958. Networks and Centres of Integration in Indian Civilization. Journal of Social Research 1 (1): 1-9.

Coleman, J.S. 1988. Social Capital in the Creation of Human Capital. The American Journal of Sociology 94: Supplement S95-S120.

Coleman, J.S. 1990. Foundations of Social Theory. Cambridge, MA: Harvard University Press.

Commons, J.R. 1924. The Legal Foundations of Capitalism. New York: Macmillan.

Constant, E.W. 2000. Recursive Practice and the Evolution of Technological Knowledge. In Technological Innovation as an Evolutionary Process, edited by J. Ziman. Cambridge, UK and New York: Cambridge University Press.

Converse, J.M., and S. Presser. 1986. Survey Questions: Handcrafting the Standardized Questionnaire: Sage Publications Inc. 
Cooke, P. 1998. Introduction. Origins of the Concept. In Regional Innovation Systems, edited by H.-J. Braczyk, P. Cooke and M. Heidenreich. London: Routledge.

Cooke, P., M.G. Uranga, and G. Etxebarria. 1998. Regional Systems of Innovation: An Evolutionary Perspective. Environment and Planning A 30: 1563-1584.

Coriat, B., and O. Weinstein. 2002. Organizations, Firms and Institutions in the Generation of Innovation. Research Policy 31 (2): 273-290.

Cowan, R. 1990. Nuclear Power Reactors: A Study in Technological Lock-In. Journal of Economic History 50 (3): 541-67.

Cowan, R. 1991. Tortoises and Hares: Choice among Technologies of Unknown Merit. Economic Journal 101 (407): 801-14.

Cowan, R., P.A. David, and D. Foray. 2000. The Explicit Economics of Knowledge Codification and Tacitness. Industrial and Corporate Change 9 (2): 211-253.

Cowan, R., and P. Gunby. 1996. Sprayed to Death: Path Dependence, Lock-in and Pest Control Strategies. Economic Journal 106 (436): 521-42.

Cowan, R., and S. Hulten. 1996. Escaping Lock-In: The Case of the Electric Vehicle. Technological Forecasting and Social Change 53: 61-79.

Cowan, R., and N. Jonard. 2006. Structural Holes, Innovation and the Distribution of Ideas Working Paper 39: United Nations University, Maastricht Economic and social Research and training centre on Innovation and Technology.

D'Exelle, B., and A. Riedl. 2008. Network Content and Directed Generosity in Real Social and Economic Networks. mimeo: University of Antwerp and Maastricht University.

Dasgupta, P., and I. Serageldin, eds. 1999. Social Capital: A Multifaceted Perspective. Washington DC: The World Bank.

David, P.A. 1985. Clio and Economics of Qwerty. American Economic Review 75 (2): 332337.

David, P.A. 1990. The Dynamo and the Computer: An Historical Perspective on the Modern Productivity Paradox. AEA Papers and Proceedings 80 (2): 335-361.

David, P.A., and G. Wright. 1999. Early Twentieth Century Productivity Growth Dynamics: An Inquiry into the Economic History of "Our Ignorance". Discussion papers in economic and social history 33: Nuffield College, University of Oxford.

Davis, K. 1949. Human Society. New York: Macmillan.

DiMaggio, P., and H. Louch. 1998. Socially Embedded Consumer Transactions: For What Kinds of Purchases Do People Most Often Use Networks? American Sociological Review 63: 619-37.

Doloreux, D., and S. Parto. 2005. Regional Innovation Systems: Current Discourse and Unresolved Issues. Technology in Society 27 (2): 133-153.

Dosi, G. 1982. Technological Paradigms and Technological Trajectories: A Suggested Interpretation of the Determinants and Direction of Technical Change. Research Policy 11: 147-162. 
Douthwaite, B., J. Keatinge, and J.R. Park. 2001. Why Promising Technologies Fail: The Neglected Role of User Innovation During Adoption. Research Policy 30: 819-836.

Dumont, L. 1970. Homo Hierachicus. Cambridge, UK: Cambridge University Press.

Dumont, L., and D. Pocock. 1957. For a Sociology of India. Contributions to Indian Sociology 1: 7-22.

Edquist, C., and B. Johnson. 1997. Institutions and Organizations in Systems of Innovation. In Systems of Innovation: Technologies, Institutions and Organizations, edited by C. Edquist. London: Pinter.

Eigen, M. 1971. 'Self-Organisation of Matter and the Evolution of Biological MacroMolecules. Naturwissenschaften 58: 465-523.

Ettlinger, N. 1994. The Localization of Development in Comparative Perspective. Economic Geography 70: 144-166.

Etzkowitz, H., and L. Leydesdorff. 2000. The Dynamics of Innovation: From National Systems And "Mode 2" To a Triple Helix of University-Industry-Government Relations. Research Policy 29: 109-123.

Evans, P. 1995. Embedded Autonomy: States and Industrial Transformation. Princeton: Princeton University Press.

Evans, P. 1996. Government Action, Social Capital and Development: Reviewing the Evidence on Synergy. World Development 24 (6): 1119-1132.

Everett, M.G., and S.P. Borgatti. 1999. Peripheries of Cohesive Subsets. Social Networks 21: 397-407.

Fakih, M., T. Rahardjo, M. Pimbert, A. Sutoko, D. Wulandari, and T. Prasetyo. 2003. Community Integrated Pest Management in Indonesia: Institutionalising Participation and People Centred Approaches, Institutionalising Participation Series. London, UK: IIED.

Farrington, J., and A.M. Martin. 1988. Farmer Participatory Research: A Review of Concepts and Recent Fieldwork. Agricultural Administration \& Extension 29: 247-264

Fine, B. 2001. Social Capital Versus Social Theory: Political Economy and Social Science at the Turn of the Millennium. London: Routledge.

Flap, H., and B. Völker. 2001. Goal Specific Social Capital and Job Satisfaction: Effects of Different Types of Networks on Instrumental and Social Aspects of Work. Social Networks 23: 297-320.

Fordham Norr, K. 1976. Factions and Kinship: The Case of a South Indian Village. Asian Survey 16 (12): 1139-1150.

Forrester, J.W. 1968a. Market Growth as Influenced by Capital Investment. Industrial Management Review 9 (2): 83-115.

Forrester, J.W. 1968b. Principles of Systems, Wright-Allen Series. Cambridge, MA: The MIT Press.

Foster, W.T. 1922. The Circuit Flow of Money. The American Economic Review 12 (3): 460-473. 
Freeman, C. 1987. Technology Policy and Economic Performance: Lessons from Japan. London and New York: Pinter Publishers.

Freeman, C. 1995. The National System of Innovation in Historical Perspective. Cambridge Journal of Economics 19 (1): 5-24.

Freeman, C. 2001. A Hard Landing for the 'New Economy'? It and the Us National System of Innovation. Structural Change and Economic Dynamics 12 (2): 115-39.

Freeman, L. 1979. Centrality in Social Networks: Conceptual Clarification. Social Networks 1: 215-239.

Frenken, K. 2000. A Complexity Approach to Innovation Networks. The Case of the Aircraft Industry (1909-1997). Research Policy 29 (2): 257-272.

Frenken, K. 2001. Understanding Product Innovation Using Complex Systems Theory, Ph.D. Thesis. Amsterdam: University of Amsterdam.

Fujita, M., P. Krugman, and A. Venables. 1999. The Spatial Economy: Cities, Regions, and International Trade. Cambridge, MA: MIT Press.

Galanakis, K., Y. Baback, and S. Passey. 2000. An Innovation Systems Model, Using the Systems Thinking Approach. 4th International Conference on Technology Policy and Innovation. Curitiba, Brazil.

Geels, F. 2002. Technological Transitions and System Innovations: A Co-Evolutionary and SocioTechnical Analysis: Blackwell Publishing.

Geertz, C. 1963. Peddlers and Princes : Social Change and Economic Modernization in Two Indonesia Towns. Chicago: University of Chicago Press.

Gell, A. 1982. The Market Wheel: Symbolic Aspects of an Indian Tribal Market. Man 17 (3): 470-491.

Gerlagh, R., and M.W. Hofkes. 2002. Escaping Lock-In: The Scope for a Transition toward Sustainable Economic Growth. Nota di lavoro 12.2002. Milan: Fondazione Eni Enrico Mattei.

Gersick, C. 1991. Revolutionary Change Theories: A Multilevel Exploration of the Punctuated Equilibrium Paradigm. Academy of Management Review 16 (1): 10-36.

Ghosh, J. 2004. Detoxifying the Villages. Frontline 21 (22).

Ghosh, J. 2005. The Political Economy of Farmer Suicides in India. Freedom from Hunger Lecture Series. New Delhi: India International Centre.

Giuliani, E. 2005 The Structure of Cluster Knowledge Networks: Uneven and Selective, Not Pervasive and Collective 05-11: DRUID Working Paper

Godin, B. 2005. The Linear Model of Innovation: The Historical Construction of an Analytical Framework. Working Paper No. 30. Montreal, Canada: Project on the History and Sociology of S\&T Statistics

Goldman, A.S. 1959. Information Flow and Worker Productivity. Management Science 5 (3): 270-278.

Goldstein, M., and C. Udry. 2004. Gender, Power and Agricultural Investment in Ghana. London, UK: London School of Economics.

Gonzalez-Brambila, C.N., F. Veloso, and D. Krackhardt. 2006. Social Capital and the Creation of Knowledge. mimeo. 
Gould, R. 1990. Power and Social Structure in Community Elites. Social Forces 68 (2): 531-552.

Gould, R.V. 1993. Collective Action and Network Structure. American Sociological Review 58 (2): 182-196.

Gould, R.V., and R.M. Fernandez. 1989. Structures of Mediation: A Formal Approach to Brokerage in Transaction Networks. Sociological Methodology 19: 89-126.

Gould, S.J., and N. Eldredge. 1993. Punctuated Equilibrium Comes of Age. Nature 366 (6452): 223-227.

Granovetter, M. 1973. The Strength of Weak Ties. The American Journal of Sociology 78 (6): 1360-1380.

Granovetter, M. 1985. Economic Action and Social Structure: The Problem of Embeddedness. The American Journal of Sociology 91 (3): 481-510.

Granovetter, M. 2005. The Impact of Social Structure on Economic Outcomes. Journal of Economic Perspectives 19 (1): 33-50.

Green, M.B. 1987. Corporate-Merger-Defined Core-Periphery Relations for the United States. Growth and Change 18 (3): 12-35.

Grossman, G.M., and E. Helpman. 1991. Innovation and Growth in the Global Economy: MIT Press.

Gulati, R. 1998. Alliances and Networks. Strategic Management Journal 19: 293-317.

Gupta, D. 2005. Whither the Indian Village - Culture and Agriculture in 'Rural' India. Economic and Political Weekly 40 (8): 751-758.

Habib, I. 1995. Essays in Indian History - Towards a Marxist Perception. New Delhi, India: Tulika Books.

Hall, A. 2006. Public-Private Sector Partnerships in an Agricultural System of Innovation: Concepts and Challenges. International Journal of Technology Management and Sustainable Development 5 (1): 3-20.

Hall, A., R.V. Sulaiman, N.G. Clark, and B. Yoganand. 2003. From Measuring Impact to Learning Institutional Lessons: An Innovation Systems Perspective on Improving the Management of International Agricultural Research. Agricultural Systems 78: 213-241.

Hall, A.J., B. Yoganand, R.V. Sulaiman, R. Raina, S. Prasad, G. Naik, and N.G. Clark, eds. 2004. Innovations in Innovation: Reflections on Partnership and Learning. Patancheru and New Delhi, India.: ICRISAT and NCAP.

Hansen, M.T. 1999. The Search-Transfer Problem: The Role of Weak Ties in Sharing Knowledge across Organizational Subunits. Administrative Science Quarterly 44 (1): 82-111.

Helpman, E., ed. 1998. General Purpose Technologies and Economic Growth. Cambridge, MA: MIT Press.

Henderson, R.M., and K.B. Clark. 1990. Architectural Innovation: The Reconfiguration of Existing Product Technologies and the Failure of Established Firms. Administrative Science Quarterly 35 (1): 9-30. 
Hirschman, A. 1958. The Strategy of Economic Development. New Haven, CN: Yale University Press.

Hirschman, A. 1977. A Generalized Linkage Approach to Development, with Special Reference to Staples. Economic Development and Cultural Change 25: 67-98.

Hirschman, A. 1992. Rival Views of Market Society and Other Recent Essays. Cambridge, MA: Harvard University Press.

Hlebec, V., and A. Ferligoj. 2002. Reliability of Social Network Measurement Instruments. Field Methods 14 (3): 288-306.

Hounshell, D.A. 1984. From the American System to Mass Production 1800-1932. Baltimore, MD: Johns Hopkins University Press.

Hounshell, D.A., and J.K. Smith. 1988a Science and Corporate Strategy: Du Pont R\&D, 1902-1980. Cambridge and New York: Cambridge University Press.

Hounshell, D.A., and J.K. Smith. 1988b. The Nylon Drama. Invention and Technology (Fall): 40-55.

Hughes, T.P. 1983. Networks of Power: Electrification in Western Society 1880-1930. Baltimore, MD: Johns Hopkins University Press.

Hughes, T.P. 1987. The Evolution of Large Technological Systems. In The Social Construction of Technological Systems, edited by W. E. Bijker, T. P. Hughes and T. Pinch. Cambridge, MA: MIT Press.

Hunter, F. 1953. Community Power Structure. A Study of Decision Makers: UNC Press.

IBRD/WB. 2006. Enhancing Agricultural Innovation: How to Go Beyond the Strengthening of Research Systems. Washington DC: The World Bank.

Irwin, M.D., and H.L. Hughes. 1992. Centrality and the Structure of Urban Interaction: Measures, Concepts, and Applications. Social Forces 71 (1): 17-51.

Jain, S., and S. Krishna. 2002. Graph Theory and the Evolution of Autocatalytic Networks. In Handbook of Graphs and Networks, edited by S. Bornholdt and H. G. Schuster: John Wiley and VCH.

Jha, S. 2004. Panchayats - Functions, Responsibilities and Resources. Mumbai: Indira Gandhi Institute of Development Research (IGIDR).

Johnson, B. 2008. How One Clumsy Ship Cut Off the Web for 75 Million People. The Guardian.

Joshi, S. 2004. Village Punukula's Different. Down to Earth.

Kaldor, N. 1972. The Irrelevance of Equilibrium Economics. Economic Journal 82: $1237-$ 55.

Kauffman, S.A. 1993. The Origins of Order. New York and Oxford, UK: Oxford University Press.

Kaufmann, A., and F. Tödtling. 2001. Science-Industry Interaction in the Process of Innovation: The Importance of Boundary-Crossing between Systems. Research Policy 30: 791-804.

Kemp, R., K. Smith, and G. Becher. 2000. How Should We Study the Relationship between Environmental Regulation and Innovation? Final Report of project, "Methodological approaches to regulation and innovation studies." EU DGIII IPTS. 
Kenney, M. 2001. The Temporal Dynamics of Knowledge Creation in the Information Society. In Knowledge Emergence: Social, Technical, and Evolutionary Dimensions of Knowledge Creation, edited by I. Nonaka and T. Nishiguchi. New York: Oxford University Press.

Kirman, A., and M. Teschl. 2006. Searching for Identity in the Capability Space. Journal of Economic Methodology 13 (3): 299-325.

Knight, F. 1933 [1951]. Economic Organisation. New York: Augustus M. Kelley.

Kodama, F. 1991. Analyzing Japanese High Technologies: The Techno-Paradigm Shift. London: Pinter Publishers.

Kodama, F. 1992. Technology Fusion and the New R\&D. Harvard Business Review (July/August).

Kogut, B. 1988. Joint Ventures: Theoretical and Empirical Perspectives. Strategic Management Journal 9: 319-332.

Kogut, B., and U. Zander. 1992. Knowledge of the Firm, Combinative Capabilities, and the Replication of Technology. Organization Science 3 (3): 383-397.

Kogut, B., and U. Zander. 1996. What Firms Do? Coordination, Identity, and Learning. Organization Science 7 (2): 502-518.

Krackhardt, D. 1987. QAP Partialling as a Test of Spuriousness. Social Networks 9: 171186.

Krackhardt, D. 1988. Predicting with Networks: Nonparametric Multiple Regression Analysis of Dyadic Data. Social Networks 10: 359-381.

Krippner, G.R. 2001. The Elusive Market: Embeddedness and the Paradigm of Economic Sociology. Theory and Society 30 (6): 775-810.

Krugman, P. 1991. Increasing Returns and Economic Geography. Journal of Political Economy 99 (3): 483-499.

Kuhn, T.S. 1970. The Structure of Scientific Revolutions. 2nd ed. Chicago, IL: University of Chicago Press.

Kuruganti, K. 2004a. No Pesticides, More Profits! The Hindu Business Line.

Kuruganti, K. 2004b. An Indian Village Says 'No' to Pesticides. peopleandplanet.net.

Lakatos, I. 1978. The Methodologies of Scientific Research Programmes: Philosophical Papers Volume 1. Cambridge, UK: Cambridge University Press.

Latour, B. 1996. Aramis, or the Love of Technology. Cambridge, MA: Harvard University Press.

Laumann, E., and F.U. Pappi. 1976. Networks of Collective Action: A Perspective on Community Influence Systems. New York: Academic Press.

Laumann, E.O., P.V. Marsden, and J. Galaskiewicz. 1977. Community-Elite Influence Structures: Extension of a Network Approach. The American Journal of Sociology 83 (3): 594-631.

Lave, J., and E. Wenger. 1991. Situated Learning. Legitimate Peripheral Participation. Cambridge, UK: Cambridge University Press. 
Lawless, M.W., and P.C. Anderson. 1996. Generational Technological Change: Effects of Innovation and Local Rivalry on Performance. The Academy of Management Journal 39 (5): 1185-1217.

Layton, E.T. 1978. Millwrights and Engineers, Science and Social Roles, and the Evolution of the Turbine in America. In The Dynamics of Science and Technology: Social Values, Technical Norms and Scientific Criteria in the Development of Knowledge, edited by W. Krohn, E. T. Layton and P. Weingart. Dordrecht, NL: Reidel.

Leffler, A., R.S. Krannich, and D.L. Gillespie. 1986. Contact, Support, and Friction: Three Faces of Networks in Community Life. Sociological Perspectives 29 (3): 337-355.

Levine, D. 2001. At the Dawn of Modernity: Biology, Culture, and Material Life in Europe. Oxford, UK: Oxford University Press.

Levinthal, D.A. 1998. The Slow Pace of Rapid Technological Change: Gradualism and Punctuation in Technological Change. Industrial and Corporate Change 7.

Lie, J. 1991. Embedding Polanyi's Market Society. Sociological Perspectives 34: 219-235.

Lin, N. 2000. Inequality in Social Capital. Contemporary Sociology 29 (6): 785-795.

Lin, N. 2001. Social Capital: A Theory of Social Structure and Action. Cambridge, UK: Cambridge University Press.

Lin, N., and M. Dumin. 1986. Access to Occupations through Social Ties. Social Networks 8: 365-385.

Lipsey, R., C. Bekar, and K. Carlaw. 1998. What Requires Explanation? In General Purpose Technologies and Economic Growth, edited by E. Helpman. Cambridge, MA: MIT Press.

Lundvall, B.A. 1985. Product Innovation and User-Producer Interaction. Aalborg, Denmark: Aalborg University Press.

Lundvall, B.A. 1988. Innovation as an Interactive Process: From User-Producer Interactions to the National System of Innovation. In Technical Change and Economic Theory, edited by G. Dosi, C. Freeman, R. Nelson, G. Silverberg and L. Soete. London: Pinter Publishers.

Lundvall, B.A., ed. 1992. National Systems of Innovation: Towards a Theory of Innovation and Interactive Learning. London: Pinter Publishers.

Lundvall, B.A., B. Johnson, E.S. Andersen, and B. Dalum. 2002. National Systems of Production, Innovation and Competence Building. Research Policy 31 (2): 213231.

Mackenzie, D. 1996. Knowing Machines: Essays on Technical Change. Cambridge, MA: MIT Press.

Mackinnon, D., K. Chapman, and A. Cumbers. 2004. Networking, Trust and Embeddedness Amongst SMEs in the Aberdeen Oil Complex. Entrepreneurship \& Regional Development 16 (2): 87 - 106.

Maital, S. 1972. The Tableau Economique as a Simple Leontief Model: An Amendment. The Quarterly Journal of Economics 86 (3): 504-507.

Mankiw, N., and M. Taylor. 2006. Economics London Thompson. 
Mansfield, E. 1963. Intrafirm Rates of Diffusion of an Innovation. The Review of Economics and Statistics 45 (4): 348-359.

Marriot, M. 1969. Review of Homo Hierarchicus: Essai Sur Le Système Des Castes by Louis Dumont. American Anthropologist, New Series 71 (6): 1166-1175.

Marriot, M. 1998. India without Hindu Concepts. The Journal of the American Oriental Society 118 (3): 377-380.

Marriott, M. 1976. Interpreting Indian Society: A Monistic Alternative to Dumont's Dualism. The Journal of Asian Studies 36 (1): 189-195.

Marsden, P.V. 1982. Brokerage Behaviour in Restricted Exchange Networks. In Social Structures and Network Analysis, edited by P. V. Marsden and L. Nin. Beverly Hills, CA: Sage.

Marsden, P.V. 1990. Network Data and Measurement. Annual Review of Sociology 16: 435-463.

Marx, K. 1867 [1976]. Capital. Vol. 1. Middlesex, UK: Penguin Books.

McEvily, B., and A. Zaheer. 1999. Bridging Ties: A Source of Firm Heterogeneity in Competitive Capabilities. Strategic Management Journal 20: 1133-56.

McGinn, K.L., and A. Keros. 2002. Improvisation and the Logic of Exchange in Embedded Negotiations. Administrative Science Quarterly 47: 442-473.

Meiers, P. Manufacture of Aluminium 2006 [cited. Available from http://www.fluoridehistory.de.

Mendelsohn, O. 1993. The Transformation of Authority. Modern Asian Studies 27 (4): 805-842.

Merrill-Sands, D.M., S.D. Biggs, R.J. Bingen, P.T. Ewell, J.L. McAllister, and S.V. Poats. 1991. Institutional Considerations in Strengthening on-Farm ClientOriented Research in National Agricultural Research Systems: Lessons from a Nine-Country Study. Experimental Agriculture 27: 343-373.

Metcalfe, J.S. 2000. Co-Evolution of Systems of Innovation. Prospects and Challenges for Research on Innovation. Berlin: Volkswagen Foundation.

Mines, M., and V. Gourishankar. 1990. Leadership and Individuality in South Asia: The Case of the South Indian Big-Man. The Journal of Asian Studies 49 (4): 761 786.

Mitchell, J.C. 1974. Social Networks. Annual Review of Anthropology 3: 279-299.

Mokyr, J. 2002. The Gifts of Athena - Historical Origins of the Knowledge Economy. Princeton, NJ Princeton University Press.

Moody, J., and D.R. White. 2003. Structural Cohesion and Embeddedness: A Hierarchical Concept of Social Groups. American Sociological Review 68 (1): 103127.

Mosse, D. 2004. Is Good Policy Unimplementable? Reflections on the Ethnography of Aid Policy and Practice. Development and Change 35 (4): 639-671.

Mosse, D. 2005. Cultivating Development: An Ethnography of Aid Policy and Practice. London: Pluto Press. 
Mowery, D.C., and R. Nelson, eds. 1999. Sources of Industrial Leadership : Studies of Seven Industries. Cambridge, UK: Cambridge University Press.

Mruthyunjaya, and P. Ranjitha. 1998. The Indian Agricultural Research System: Structure, Current Policy Issues, and Future Orientation. World Development 26 (6): 1089-1101.

Mumford, L. 1934. Technics and Civilization. New York: Harcourt Brace and Company.

Murmann, J.P. 2001. New Directions in Research on Dominant Design, Technological Innovations and Industrial Change. Kellogg graduate school of management, Northwestern University. Evanston, IL.

Musson, A.E. 1976. Industrial Motive Power in the United Kingdom, 1800-70. Economic History Review 29 (3): 415-439.

Myrdal, G. 1957. Economic Theory and Underdeveloped Regions. London: Gerald Duckworth.

Nelson, R., ed. 1993. National Innovation Systems: A Comparative Analysis. New York and Oxford: Oxford University Press.

Nelson, R.R., and S.G. Winter. 1977. In Search of a Useful Theory of Invention. Research Policy 6: 36-76.

Nelson, R.R., and S.G. Winter. 1982. An Evolutionary Theory of Economic Change. Cambridge, MA: Harvard University Press.

Newcomb, S. 1886. Principles of Political Economy. New York Harper \& Brothers.

Nightingale, P. 1998. A Cognitive Theory of Innovation. Research Policy 27: 689-709.

Nightingale, P. 2003. If Nelson and Winter Are Only Half Right About Tacit Knowledge, Which Half? A Searlean Critique of 'Codification'. Industrial and Corporate Change 12 (2): 149-183.

Noble, D.F. 1984. Forces of Production: A Social History of Industrial Automation. Oxford, UK: Oxford University Press.

Nordhaus, W. 1992. The Ecology of Markets. Paper read at National Academy of Sciences of the United States of America, February 1st 1992, at Washington, DC.

O’Hearn, D. 1994. Innovation and the World-System Hierarchy: British Subjugation of the Irish Cotton Industry, 1780-1830. American Journal of sociology 100 (3): 587621.

Olson, M. 1971. The Logic of Collective Action: Public Goods and the Theory of Groups. Cambridge, MA: Harvard University Press.

Oommen, T.K. 1970. Rural Community Power Structure in India. Social Forces 49 (2): 226-239.

Orsenigo, L., F. Pammolli, and M. Riccaboni. 2001. Technological Change and Network Dynamics: Lessons from the Pharmaceutical Industry. Research Policy 30 (3): 485-508.

Özman, M. 2005. Networks, Organizations and Knowledge, MERIT, Universiteit Maastricht, The Netherlands. 
Pal, S., and D. Byerlee. 2003. The Funding and Organisation of Agricultural Research in India: Evolution and Emerging Policy Issues 16. New Delhi, India: National Centre for Agricultural Economics and Policy Research, Indian Council of Agricultural Research.

Paldam, M. 2005. Social Capital and Social Policy. Paper presented at the conference: New Frontiers of Social Policy. Arusha.

Parto, S. 2005. Economic Activity and Institutions: Taking Stock. Journal of Economic Issues 39 (1): 31-52.

Parto, S., T. Ciarli, and S. Arora. 2005. Economic Growth, Innovation Systems, and Institutional Change: A Trilogy in Five Parts. Research Memorandum 021. Maastricht: MERIT.

Pasinetti, L.L. 1980. 'the Notion of Vertical Integration in Economic Analysis' In Essays on the Theory of Joint Production, edited by L. L. Pasinetti. Woking, UK: Macmillan.

Patinkin, D. 1973. In Search of The "Wheel of Wealth": On the Origins of Frank Knight's Circular-Flow Diagram. The American Economic Review 63 (5): 1037 1046.

Patten, B.C., and E.P. Odum. 1981. The Cybernetic Nature of Ecosystems. The American Naturalist 118: 886-895.

Pistorius, C.W.I., and J.M. Utterback. 1997. Multi-Mode Interactions among Technologies. Research Policy 26 (1): 67-84.

Podolny, J., and J. Baron. 1997. Resources and Relationships: Social Networks and Mobility in the Workplace. American Sociological Review 62 (5): 673-693.

Podolny, J., and K. Page. 1998. Network Forms of Organization. Annual Review of Sociology 24: 57-76.

Polanyi, K. 1944. The Great Transformation: The Political and Economic Origins of Our Time. Boston, MA: Beacon Press.

Polanyi, K. 1957 [1992]. The Economy as Instituted Process. In The Sociology of Economic Life, edited by M. Granovetter and R. Swedberg. Boulder, CO: Westview Press.

Polmear, I.J. 2004. Aluminium Alloys - a Century of Age Hardening Materials Forum 28.

Portes, A., and J. Sensenbrenner. 1993. Embeddedness and Immigration: Notes on the Social Determinants of Economic Action. The American Journal of Sociology 98 (6): 1320-50.

Prasad, S. 2005. Science and Technology in Civil Society: Innovation Trajectory of Spirulina Algal Technology. Economic and Political Weekly 40 (40): 4363-72.

Putnam, R. 1993. The Prosperous Community: Social Capital and Public Life. American Prospect, 35-42.

Putnam, R. 2000. Bowling Alone: The Collapse and Revival of American Community. New York: Simon and Schuster. 
Putnam, R., R. Leonardi, and R.Y. Nanetti. 1993. Making Democracy Work: Civic Traditions in Modern Italy. Princeton: Princeton University Press.

Quigley, D. 1993. The Interpretation of Caste. Oxford, UK: Clarendon Press.

Raheja, G.G. 1988. The Poison in the Gift: Ritual Prestation and the Dominant Caste in a North Indian Village. Chicago: University of Chicago Press.

Ramanjaneylu, G.V., K. Kuruganti, Z. Hussain, and K. Venumadhav. 2004. No Pesticides, No Pests. Hyderabad, AP: Centre for Sustainable Agriculture.

Ramanjaneylu, G.V., K. Kuruganti, Z. Hussain, and K. Venumadhav. 2005. Managing Pests without Pesticides. Hyderabad: Centre for Sustainable Agriculture.

Rao, D.C.B. 2004. Village Bids Farewell to Pesticides. The Hindu.

Reddy, G.R., and G. Haragopal. 1985. The Pyraveekar: "the Fixer" in Rural India. Asian Survey 25 (11): 1148-1162.

Richardson, G.B. 1972. The Organisation of Industry. Economic Journal 82: 883-96.

Richardson, G.B. 1995. The Theory of the Market Economy. Revue économique 46 (6): 1487-1496.

Rip, A., and R. Kemp. 1998. Technological Change. In Human Choice and Climate Change, edited by S. Rayner and L. Malone. Washington, DC: Batelle Press.

Rodan, S., and C. Galunic. 2004. More Than Network Structure: How Knowledge Heterogeneity Influences Managerial Performance and Innovativeness. Strategic Management Journal 25: 541-562.

Romanelli, E., and M.L. Tushman. 1994. Organizational Transformation as Punctuated Equilibrium: An Empirical Test. The Academy of Management Journal 37 (5): 1141-1166.

Rosenberg, N. 1976. Perspectives of Technology. Cambridge, UK: Cambridge University Press.

Rosenberg, N. 1982. Inside the Black Box: Technology and Economics. Cambridge: Cambridge University Press.

Rosenberg, N. 1996. Uncertainty and Technological Change. In The Mosaic of Economic Growth, edited by R. Landau, T. Taylor and G. Wright. Stanford, CA: Stanford University Press.

Rothwell, R. 1992. Successful Industrial Innovation: Critical Factors for the 1990s R\&D Management 22 (3): 221-240.

Rowley, T., D. Behrens, and D. Krackhardt. 2000. Redundant Governance Structures: An Analysis of Structural and Relational Embeddedness in the Steel and Semiconductor Industries. Strategic Management Journal 21: 369-86.

Sahal, D. 1981. Alternative Conceptions of Technology. Research Policy 10: 2-24.

Sahal, D. 1985. Technological Guideposts and Innovation Avenues. Research Policy 14: 61-82.

Samuelson, P.A. 1970 Economics: An Introductory Analysis. 8th edition ed. New York: McGraw-Hill.

Saxenian, A. 1994. Regional Advantage: Culture and Competition in Silicon Valley and Route 128. Cambridge, MA: Harvard University Press. 
Schmitz, H., and K. Nadvi. 1999. Clustering and Industrialization: Introduction. World Development 27 (9): 1503-1514.

Schumpeter, J.A. 1912 [1934]. The Theory of Economic Development. Cambridge, MA: Harvard University Press.

Schumpeter, J.A. 1954. History of Economic Analysis. London: Allen\&Unwin.

Scott, W.R. 2001. Institutions and Organizations. 2nd ed. London: Sage Publications.

Sechrest, L., M. Stewart, and M. Stickle. 1998. Factors Affecting the Adoption and Impact of CGIAR Innovations: A Synthesis of Findings. Washington: IAEG, CGIAR.

Shannon, C.E., and W. Weaver. 1949. The Mathematical Theory of Communication. Urbana, IL: University of Illinois

Sherwood, S., and S. Larrea. 2001. Looking Back to See Ahead: Farmer Lessons and Recommendations after 15 Years of Innovation and Leadership in Güinope, Honduras. Agriculture and Human Values 18 (2): 195-208.

Shiva, V. 2004. The Suicide Economy of Corporate Globalization. wnw.countercurrents.org/glo-shiva050404.htm.

Silverberg, G., and B. Verspagen. 2004. A Percolation Model of Innovation in Complex Technology Space. Journal of Economic Dynamics and Control 29 (1-2): 225-244.

Simon, H.A. 1981. The Sciences of the Artificial. Cambridge, MA: MIT Press.

Smith, A. 1776 [1991]. Wealth of Nations. New York: Prometheus Books.

Spengler, J.J. 1945. The Physiocrats and Say's Law of Markets. I. The Journal of Political Economy 53 (3): 193-211.

Spielman, D.J. 2005. Innovation Systems Perspectives on Developing-Country Agriculture: A Critical Review Washington, DC: International Food Policy Research Institute.

Sraffa, P. 1960. Production of Commodities by Means of Commodities. Cambridge, UK: Cambridge University Press.

Srinivas, M.N. 1955. The Social System of a Mysore Village. In Village India edited by M. Marriott. Chicago: University of Chicago Press.

Srinivas, M.N. 1959. The Dominant Caste in Rampura. American Anthropologist, New Series 61 (1): 1-16.

Srinivas, M.N. 1975. The Indian Village: Myth and Reality. In Studies in Social Anthropology: Essays in Memory of Sir E.E Evans-Pritchard, edited by J. H. M. Beattie and G. Lienhardt. Oxford, UK: Oxford University Press.

Srinivas, M.N., and A. Beteille. 1964. Networks in Indian Social Structure. Man 64: 165-168.

Stokman, F. 2004. What Binds Us When with Whom: Content and Structure in Social Network Analysis. SUNBELT XXIV: International Social Networks Conference. Portoroz, Slovenia.

Suarez, F.F., and J.M. Utterback. 1995. Dominant Designs and the Survival of Firms. Strategic Management Journal 16 (6): 415-430. 
Swedberg, R. 1997. New Economic Sociology: What Has Been Accomplished, What Is Ahead? Scandinavian Sociological Association 40 (2): 161-182.

Szreter, S. 2002. The State of Social Capital: Bringing Back in Power, Politics, and History. Theory and Society 31 (5): 573-621.

Thomke, S.H. 2001. The Impact of Technology on Knowledge Creation: A Study of Experimentation in Integrated Circuit Design In Knowledge Emergence. Social, Technical, and Evolutionary Dimensions of Knowledge Creation edited by Nonaka and Nishiguchi, New York: Oxford University Press.

Thompson, J., and I. Scoones. 1994. Beyond Farmer First : Rural People's Knowledge, Agricultural Research and Extension Practice London, UK: Intermediate Technology Publications.

Thorner, D. 1954. The Village Panchayat as a Vehicle of Change. Economic Development and Cultural Change 2 (3): 209-215

Thrupp, L.A. 1989. Legitimizing Local Knowledge: From Displacement to Empowerment for Third World People. Agriculture and Human Values: 13-24.

Tirole, J. 1988. The Theory of Industrial Organization. Cambridge, MA: MIT Press.

Tushman, M., and P. Murmann. 1998. Dominant Designs, Innovation Types and Organizational Outcomes. Research in Organizational Behavior 20.

Tushman, M.L., and P. Anderson. 1986. Technological Discontinuities and Organizational Environments. Administrative Sciences Quarterly 31: 439-465.

Tushman, M.L., and L. Rosenkopf. 1992. Organizational Determinants of Technological Change: Toward a Sociology of Technological Evolution. Research in Organizational Behavior 14: 311-347.

Udry, C., and T.G. Conley. 2004. "Social Networks in Ghana" 888: Economic Growth Centre, Yale University.

Unruh, G.C. 2000. Understanding Carbon Lock-In. Energy Policy 28 (12): 817-830.

Uphoff, N., ed. 2002. Agroecological Innovations : Improving Food Production with Participatory Development. London: Earthscan.

Usher, A.P. 1954. A History of Mechanical Inventions. New York: Dover Publications.

Utterback, J.M. 1994. Mastering the Dynamics of Innovation. Boston, MA: Harvard Business School Press.

Utterback, J.M., and W.J. Abernathy. 1975. A Dynamic Model of Process and Product Innovation. OMEGA, The Intl. J. of Mgmt. Sci. 3 (6): 424-441.

Uzzi, B. 1996. The Sources and Consequences of Embeddedness for the Economic Performance of Organizations: The Network Effect. American Sociological Review 61 (4): 674-98.

Uzzi, B. 1997. Social Structure and Competition in Interfirm Networks: The Paradox of Embeddedness. Administrative Science Quarterly 42: 35-67.

Uzzi, B., and R. Lancaster. 2003. Relational Embeddedness and Learning: The Case of Back Loan Managers and Their Clients. Management Science 49 (4): 383-399.

van der Gaag, M., and T.A.B. Snijders. 2005. The Resource Generator: Social Capital Quantification with Concrete Items. Social Networks 27 (1): 1-29. 
Vasavi, A.R. 1999. Harbingers of Rain: Land and Life in South India. New Delhi: Oxford University Press.

von Hippel, E. 1988. Sources of Innovation. Oxford and New York: Oxford University Press.

von Tunzelmann, G.N. 1978. Steam Power and British Industrialization to 1860. Oxford, UK: Clarendon Press.

Wade, R. 1988. Village Republics: Economic Conditions for Collective Action in South India. New York: Cambridge University Press.

Wallace, A.F.C. 1978. Rockdale: The Growth of an American Village in the Early Industrial Revolution. New York: Alfred A. Knopf.

Wallerstein, I. 1974. The Modern World System I: Capitalist Agriculture and the Origins of the European World-Economy in the Sixteenth Century. New York: Academic Press.

Wallerstein, I. 1983. Historical Capitalism. London: Verso.

Walton, J. 1966. Substance and Artifact: The Current Status of Research on Community Power Structure. The American Journal of Sociology 71 (4): 430-438.

Walton, J. 1976. "Community Power and the Retreat from Politics; Full Circle after Twenty Years?" Social Problems 23 (3): 292-303.

Walton, J. 1992. Western Times and Water Wars: State, Culture, and Rebellion in California. Berkeley, CA: University of California Press.

Wasserman, S., and K. Faust. 1994. Social Network Analysis: Methods and Applications. Cambridge, UK: Cambridge University Press.

Waters-Bayer, A., and W. Bayer. 2005. The Social Dimensions in Agricultural R\&D: How Civil Society Fosters Partnerships to Promote Local Innovation by Rural Communities. EFARD 2005 Agricultural Research for Development: European Responses to Changing Global Needs. Zurich.

Welch, B.J. 1999. Aluminum Production Paths in the New Millennium. JOM 51 (5): 24-28.

Wellman, B. 1983. Network Analysis: Some Basic Principles. Sociological Theory 1: 155200.

Wellman, B., and S.D. Berkowitz, eds. 1988. Social Structures: A Network Approach. Cambridge: Cambridge University Press.

Wellman, B., and S. Wortley. 1990. Different Strokes from Different Folks: Community Ties and Social Support. The American Journal of Sociology 96 (3): 558-588.

Wiener, N. 1948. Cybernetics or Control and Communication in the Animal and the Machine. New York: Wiley.

Williams, E. 2003. Forecasting Material and Economic Flows in the Global Production Chain for Silicon. Technological Forecasting and Social Change 70: 341-357.

Winner, L. 2002. Complexity, Trust and Terror. Tech. Knowledge Revue 3 (1).

Woolcock, M. 1998. Social Capital and Economic Development: Toward a Theoretical Synthesis and Policy Framework. Theory and Society 27 (2): 151-208. 
Wu, B., and J.N. Pretty. 2004. Social Connectedness in Marginal Rural China: The Case of Farmer Innovation Circles in Zhidan, North Shaanxi. Agriculture and Human Values 21 (1): 81-92.

Zukin, S., and P. DiMaggio. 1990. Introduction. In Structures of Capital: The Social Organization of the Economy, edited by S. Zukin and P. DiMaggio. Cambridge: Cambridge University Press. 


\section{Appendix A}

\section{Survey Questionnaires}

\section{Farm Labourer questionnaire}

\section{General Questions}

1. Names of wife and husband

2. Daily wage of wife and husband

3. Work availability in the last one year, average number of days per month? During Kharif:

During Rabi:

4. On whose land did you work frequently in the last year? Please provide the names of the landlords and their village(s).

5. What different types of farm work (such as harvesting, ploughing, weeding, etc) do you usually do?

6. In addition to farming related work, what other types of work (such as construction work, etc) did you do in the last year? During which months and where?

\section{NPM knowledge and practices}

7. Did you ever use (or prepare) NPM solutions, for someone else, in the last five years?

If yes, when did you first use NPM, on which crop, and why (please provide as many reasons as possible)?

8. Did you ever collect NPM ingredients and prepare the solutions to sell to other farmers?

If yes, when, and where did you find the ingredients?

(Ask questions 9-11 only if the response to question 7 or 8 is YES i.e., a farm labourer indicates NPM use at least once. Otherwise, go to question 12 below)

9. What different types of NPM methods and solutions do (or did) you use? Please indicate in the list below,

\footnotetext{
- $\quad$ neem seed kernel extract
} 
- cow dung and urine solution

- chilli and garlic extract

- $\quad$ tobacco, rice bran, jaggery solution

- detergent and tobacco solution

- Tulsi, marigold flowers, cow dung, urine, garlic, super potash solution

- $\quad$ powdered kodisha plant-branches

- Musti kaya (Fruit of Strichnos Nuxvomica) solution

- pheromone traps

- trapper plants such as sunflower, maize, marigold etc.

- delayed planting

- crop rotation

- $\quad$ any other solutions (including mixtures requiring the use of pesticides)

10. Did you modify any existing NPM methods/solutions or discover any new natural method (for example, a new mixture or a new trapper plant) over the course of your experience with NPM?

If yes, what did you discover, when did you first try it and with what results?

11. Did you tell any other farmers or NGO representatives about your discovery?

If yes, to whom (please provide as many names as possible)?

\section{Knowledge flows}

12. In case of some new work on the field, such as using a NPM method or a new type of pesticides, who do you ask for advice? Please select in the list below and grade the usefulness of the advice in carrying out the required task? Please provide as many names as possible.

a. Farmers and farm labourers from Punukula or other villages

Name and village:

Name and village:

Name and village:

b. NGO coordinator

Name and organization:

Name and organization:

c. Pesticide dealers

Name and shop location:

Name and shop location:

d. Anyone else (including government agriculture department extension officers)

Name and organization:

Name and organization: 
13. Who asked you for advice about this new work? Please select from the list below and in each category, provide names of the individuals, their organizations or villages.

a. Farmers and farm labourers

Name and village:

Name and village:

Name and village:

b. NGO coordinator

Name and organization:

Name and organization:

c. Pesticide dealers

Name and shop location:

Name and shop location:

d. Anyone else (including government agriculture department extension officers)

Name and organization:

Name and organization:

\section{Business Network}

14. When you need a loan, who do you approach? Please select in the list below and name the individual and his organization/village.

a. A big farmer (from this or another village)

Name and village:

Name and village:

b. A moneylender (sabukar from this or another village)

Name and village:

c. A pesticide/fertiliser dealer

Name and shop location:

d. A bank officer

Name and location:

e. Anyone else

Name and organization:

15. Where do you get your tools and other farming related equipment? Please provide the names of the individual/shop and location.

\section{$\underline{\text { Social Network }}$}

16. Please name families/individuals in this village who are your close relatives (you help each other in times of need and celebrate festivals together), but own a separate piece of land or live separately if landless. Please provide as many names as possible.

a. Name of the relative:

b. Name of the relative:

c. Name of the relative: 
d. Name of the relative:

17. Please name families/individuals in this village who are your close friends (you help each other in times of need). Please provide as many names as possible.

a. Name of the friend (family name, first name):

b. Name of the friend:

c. Name of the friend:

18. Please provide names and villages of your close friends/relatives who live outside this village but you see each other frequently.

a. Name of the friend/relative and village:

b. Name of the friend/relative and village:

c. Name of the friend/relative and village:

Final Remarks

19. Did you ever get health problems due to pesticide use while working on the field?

If yes, when, and why (please give as many reasons as possible)? Were you hospitalised, if yes, who paid the doctor's bill?

\section{Shopkeeper questionnaire}

\section{General Questions}

1. Names of wife and husband

2. What is your primary profession?

2a. What kinds of goods/service do you sell? For example, transportation for auto drivers.

3. Did you take a loan to start this business?

If yes, from whom? Please name the individual and organization.

a. Bank Officer and Bank Name

b. A big farmer

c. A moneylender (sahukar)

d. A micro-credit agency (please name the agent)

e. Anyone else (please provide details)

4. In addition to this business, what other types of work (such as farming) did you do in the last few years? Where? 
5. Do you sell any farm-inputs?

If yes, where do you buy them (please provide the name of the individual/organization for each input listed below)?

a. Seeds

b. Fertilizers

c. Neem seeds

d. Other NPM inputs such as pheromone traps

e. Pesticides

f. Any other (please mention)

Knowledge flows

6. From whom do you get information about new products that you can sell at your shop such as ingredients for NPM, or new goods/components that you can install to do your work better? Please provide as many names as possible.

a. Farmers and farm labourers

Name and village:

Name and village:

Name and village:

b. NGO coordinator

Name and organization:

Name and organization:

c. Other small business owners (shopkeepers or auto drivers etc.)

Name and shop location:

Name and shop location:

d. Anyone else

Name and organization:

Name and organization:

7. Who asks you for information/advice about new products? Please select from the list below and in each category, provide names of the individuals, their organizations or villages.

a. Farmers and farm labourers

Name and village:

Name and village:

Name and village:

b. NGO coordinator

Name and organization:

Name and organization:

c. Other small business owners (shopkeepers or auto drivers etc.)

Name and shop location:

Name and shop location:

d. Anyone else 
Name and organization:

Name and organization:

\section{Business Network}

8. When you need a loan, who do you approach? Please select in the list below and name the individual and his organization/village.

a. A big farmer (from this or another village)

b. A moneylender (sabukar from this or another village)

c. A pesticide/fertiliser dealer

d. A bank officer

e. Anyone else

9. Where do you get your supplies (or services such as any NPM ingredients, or spare parts etc. in case of auto drivers)? Please provide the names of the individuals or shops and their location (include the individuals who visit your village to sell you the supplies).

10. Do all villagers purchase from your shop (or use your service)?

If no, which sections of the village do you service more regularly? Please select in the list below.

a. People who live in your vicinity

b. Big farmers from the entire village

c. People from your own community

d. People from other villages/towns

\section{$\underline{\text { Social Network }}$}

11. Please name families/individuals in this village who are your close relatives (you help each other in times of need and celebrate festivals together), but own a separate piece of land or live separately if landless. Please provide as many names as possible.

a. Name of the relative:

b. Name of the relative:

c. Name of the relative:

d. Name of the relative:

12. Please name families/individuals in this village who are your close friends (you help each other in times of need). Please provide as many names as possible.

a. Name of the friend (family name, first name):

b. Name of the friend:

c. Name of the friend: 
13. Please provide names and villages of your close friends/relatives who live outside this village but you see each other frequently.

a. Name of the friend/relative and village:

b. Name of the friend/relative and village:

c. Name of the friend/relative and village:

\section{Final Remarks}

14. Does any SECURE coordinator provide any form of assistance to you? If yes, what help does the coordinator provide and how often does she/he visit you? Is there anything else you'd like to tell us?

\section{Questionnaire for $\mathrm{NGO}$ representatives}

\section{General Questions}

1.Name and Organization:

\section{Age:}

3. Place of present residence and native of (town/village):

4. Educational qualifications:

5. Years of employment with the present NGO:

6. Previous employer(s) and a brief description of job responsibility:

7. Brief description of present responsibilities (e.g. descriptions of projects, including names of donors, that you are involved with at present):

8. If ever involved in an NPM project, when (years) and where?

9. Have you ever worked in a project in Punukula?

If yes, please describe the project(s) and provide the years that you were active in it.

10. Please name the farmers/farm labourers that you most actively worked with in Punukula (please provide as many names as possible).

Name of the Punukula resident:

Name:

Name:

Name: 


\section{Knowledge network}

11. From whom did you receive feedback about the implementation and results of NPM project or experiments in Punukula? Please name the individuals.

Name of the Punukula resident:

Name:

Name:

Name:

12. When (season and year) was the most serious problem, such as a severe pest attack, that you encountered during the years you worked in Punukula?

13. To solve this problem, from whom do (or did) you take advice?

a. Farmers or farm labourers

Name and village:

Name and village:

b. A colleague (from the same NGO)

Name:

Name:

c. People from other NGO's

Name and organization:

Name and organization:

d. Shopkeepers such as pesticide/fertiliser dealers

Name and shop location:

e. Anyone else (including government officials)

Name and organization:

13. For solving the abovementioned problem, who took advice from you?

a. Farmers or farm labourers

Name and village:

Name and village:

Name and village:

b. A colleague (from the same NGO)

Name:

Name:

c. People from other NGO's

Name and organization:

Name and organization:

d. Shopkeepers such as pesticide/fertiliser dealers

Name and shop location:

e. Anyone else (including government officials)

Name and organization: 


\section{Business and Social Network}

14. Which of SECURE donors do you directly interact with? Please name as many individuals and their organizations (with locations) as possible.

15. Please name any close friends or relatives who work in SECURE or other organizations who are SECURE's partners or donors.

Name of friend/relative and organization:

Name of friend/relative and organization:

16. Please name any close friends and relatives who live in Punukula or other villages in the same gram panchayat.

Name of friend/relative and village:

Name of friend/relative and village:

17. Names of close friends and relatives who are farmers in other villages.

Name of farmer and village:

Name of farmer and village:

Name of farmer and village:

18. Did you come up with a new NPM method or find a new solution for any other problem that you have faced in one of your projects? If yes, please describe your new solution briefly. When did you first try it and with what results?

18a. Did you tell anyone else about this innovation? In each case below, please give the name of the concerned person and the name of her organization or village.

a. Farmers or farm labourers

b. A colleague

c. People from other NGO's

d. Shopkeepers such as pesticide/fertiliser dealers

e. Anyone else (including government officials) 


\section{Appendix B}

\section{Different types of centrality measures (Freeman 1979)}

For undirected graphs

Degree centrality

$$
C_{D, j}=\frac{\sum_{i=1}^{n} a(i, j)}{(n-1)}
$$

where $a(i, j)$ is 1 if and only if nodes $i$ and $j$ are connected by an edge (link) and 0 otherwise, $\mathrm{n}$ is the total number of nodes in the graph. Degree centrality determines the influence of a node in a network based on its direct links.

Betweenness centrality $\quad C_{B, k}=\frac{\sum_{i}^{n} \sum_{j}^{n} b_{i j}(k)}{\left(n^{2}-3 n+2\right) / 2}$

where $b_{i j}(k)=g_{i j}(k) / g_{i j}$ and $g_{i j}$ is the number of geodesics (shortest paths) connecting nodes $i$ and $j$, and $g_{i j}(k)$ is the number of geodesics connecting $i$ and $j$ that contain $k$. Between centrality is the sum of all probabilities that the geodesic between nodes $i$ and $j$ will pass through $k$. By being located on the geodesics, node $k$ is assumed to control the flow of information between nodes $i$ and $j$.

Closeness centrality

$$
C_{c, k}=\frac{n-1}{\sum_{i=1}^{n} d(i, k)}
$$

where $d(i, k)$ is the geodesic distance of node $k$ to node $i$. By taking the average of the shortest distance of a node to all other (connected) nodes in a network, closeness centrality determines its influence based on its direct and indirect links. 


\section{Appendix C}

\section{Bigger core in Punukula's social network}

This core is formed with the union of two sets, one with the fifteen highest closeness centrality values and the other with fifteen lowest network constraint (listed in Tables C.1 and C.2 respectively). The union of the two sets of fifteen people each formed a group with 22 members. However, two of these people had less than two links connecting them to other members of the group, so could not form part of a circular process (necessary for the formation of a core). The names of the final 20 people are listed in the table and the structure of the core formed by them is depicted in the figure below. The arguments on the constitution of core presented in Chapter 8 , such as most core members being community heads and/or resource persons of the local NGO in the village, are valid for the larger core presented here. The only notable difference between this core of 20 people and the smaller one of size 10 in Chapter 8 , constituted entirely of Punukula residents, is the presence of the main all-in-one dealer in the former. He is depicted as node 1 in the Figure C. 1 below.

Table C.1 List of 15 people with the highest closeness centrality

\begin{tabular}{|l|l|}
\hline Name of the people with highest values & Closeness centrality \\
\hline Gorinta, EG & 0.2608333 \\
\hline Veena, IG & 0.2507319 \\
\hline Velu, HE & 0.2443115 \\
\hline Moti, CQ & 0.2426762 \\
\hline Koli, ED & 0.2404233 \\
\hline Pillai, GB & 0.2391546 \\
\hline Pai, DJ & 0.2339086 \\
\hline Joardar, GX & 0.2333065 \\
\hline Pillai, GA & 0.2327075 \\
\hline Yarla, FQ & 0.2312234 \\
\hline Pillai, FZ & 0.2291772 \\
\hline Velu, HG & 0.2265989 \\
\hline Karai, DF & 0.2260339 \\
\hline Raj, M., A-one Fertilizers & 0.2257524 \\
\hline Valli, GG & 0.2194663 \\
\hline
\end{tabular}

Table C. 2 List of 15 people with the lowest network constraint values

\begin{tabular}{|l|l|}
\hline Name of the people with lowest values & Network constraint \\
\hline Gorinta, EG & 0.1163911 \\
\hline Sapna, FC & 0.1225137 \\
\hline Koli, ED & 0.1301974 \\
\hline
\end{tabular}




\begin{tabular}{|l|l|}
\hline Joardar, GX & 0.1342485 \\
\hline Veena, IG & 0.1439568 \\
\hline Seri, AX & 0.1479429 \\
\hline Yarla, FQ & 0.1547835 \\
\hline Moti, CQ & 0.1578836 \\
\hline Velu, HO & 0.1618403 \\
\hline Koli, EB & 0.1740398 \\
\hline Velu, HG & 0.1768314 \\
\hline Arayar, GO & 0.1778434 \\
\hline Veera, DP & 0.1790158 \\
\hline Koli, EA & 0.1832282 \\
\hline Velu, HE & 0.1842998 \\
\hline &
\end{tabular}

Table C.3 Members of the social network core of size 20

\begin{tabular}{|l|l|}
\hline Name & Node number in Figure C.1 below \\
\hline Raj, M., A-one Fertilizers & 1 \\
\hline Moti, CQ & 2 \\
\hline Karai, DF & 3 \\
\hline Pai, DJ & 4 \\
\hline Veera, DP & 5 \\
\hline Koli, EA & 6 \\
\hline Koli, EB & 7 \\
\hline Koli, ED & 8 \\
\hline Gorinta, EG & 9 \\
\hline Yarla, FQ & 10 \\
\hline Pillai, FZ & 11 \\
\hline Pillai, GA & 12 \\
\hline Pillai, GB & 13 \\
\hline Valli, GG & 14 \\
\hline Arayar, GO & 15 \\
\hline Joardar, GX & 16 \\
\hline Velu, HE & 17 \\
\hline Velu, HG & 18 \\
\hline Velu, HO & 19 \\
\hline Veena, IG & 20 \\
\hline
\end{tabular}




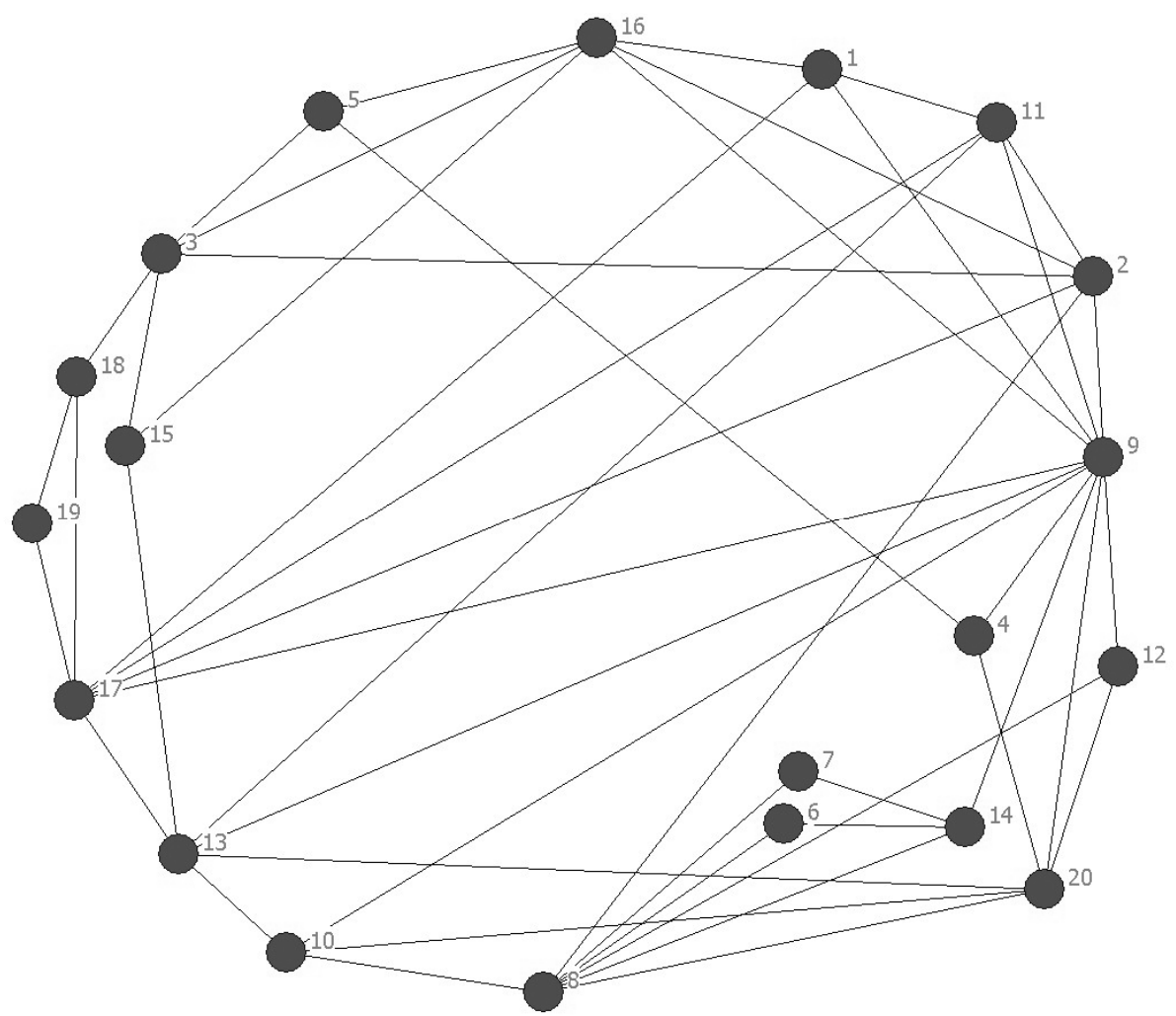

Figure C.1 Structure of links among the members of the social network core of size 20. 


\section{Appendix D}

\section{D.I Embeddedness of individual knowledge flows in indirect social ties in the local innovation system}

A total of 50 (31) people have an incoming (outgoing) knowledge link that doubles as an indirect social tie, as compared to 26 (24) knowledge links embedded in direct social relations (as shown in Chapter 9). Therefore, more people get and take problemsolving advice from friends of friends, as compared to direct friends and kin. However, one must note that the average number of indirect social ties per individual, 6.90, is greater than the average number of direct social ties (1.87, the average degree in the social network). Individuals with the highest 33 values $(\geq 0.5)$ of proportional embeddedness of incoming knowledge links in their indirect social ties are listed in Table D.1. All 31 people with nonzero embeddedness for outgoing knowledge links Table D.2. Individuals with names in italics were not personally interviewed (three in Table D.1 and none in Table D.2).

Only one person in Table D.1 has the highest number of 4 coincident links: Koli, ED. Three people have 3 incoming knowledge links that double as social ties: Pillai, GC; Gorinta, EG; and Seri, AT. These four people thus also have the highest indirect social capital for knowledge access in the networks. ${ }^{1}$ Two of these four people, ED Koli and EG Gorinta, also had the highest number of their incoming knowledge links embedded in direct social ties, as shown in Table 9.2 (Chapter 9). The last of the four GC Pillai, son of the headman of the second largest sub-caste in the village, lives in the nearby town where all the pesticide dealers are located and is a friend of the main pesticide supplier to the village, M Raj.

Table D.1 Embeddedness of incoming knowledge links in indirect social ties 'undivided' networks

\begin{tabular}{|l|l|l|l|}
\hline & I & II & III \\
\hline & $\begin{array}{l}\text { Name of the } \\
\text { individual }\end{array}$ & $\begin{array}{l}\text { Number of incoming links } \\
\text { coincident with indirect social ties }\end{array}$ & $\begin{array}{l}\text { Degree of } \\
\text { embeddedness }\end{array}$ \\
\hline 1. & Koli, ED & 4 & 0.667 \\
\hline 2. & Pillai, GC & 3 & 1 \\
\hline 3. & Gorinta, EG & 3 & 0.75 \\
\hline 4. & Seri, AT & 3 & 0.6 \\
\hline 5. & Seri, AW & 2 & 1 \\
\hline 6. & Karai, DF & 2 & 0.667 \\
\hline 7. & Nath, FG & 2 & 0.667 \\
\hline
\end{tabular}

${ }^{1}$ Indirect social capital simply reflects the number of an individual's indirect social ties (friends of friends) that she uses to access useful knowledge. 


\begin{tabular}{|c|c|c|c|}
\hline 8. & Lal, BE & 2 & 1 \\
\hline 9. & Lal, BM & 1 & 1 \\
\hline 10. & Sapna, EZ & 1 & 1 \\
\hline 11. & Sapna, FD & 1 & 1 \\
\hline 12. & Pillai, GA & 1 & 1 \\
\hline 13. & Trupti, BA & 1 & 1 \\
\hline 14. & Muthu, GD & 1 & 1 \\
\hline 15. & Joardar, GX & 1 & 1 \\
\hline 16. & Velu, HD & 1 & 1 \\
\hline 17. & Velu, HG & 1 & 1 \\
\hline 18. & Velu, HL & 1 & 1 \\
\hline 19. & Palam, HS & 1 & 1 \\
\hline 20. & Veena, IG & 1 & 1 \\
\hline 21. & \begin{tabular}{|l} 
Adnoor, Z. \\
Kumarampuram
\end{tabular} & 1 & 1 \\
\hline 22. & Subba, CA & 1 & 0.5 \\
\hline 23. & Subba, CB & 1 & 0.5 \\
\hline 24. & Naidu, CU & 1 & 0.5 \\
\hline 25. & Koli, DY & 1 & 0.5 \\
\hline 26. & Koli, EA & 1 & 0.5 \\
\hline 27. & Koli, EB & 1 & 0.5 \\
\hline 28. & Sapna, EU & 1 & 0.5 \\
\hline 29. & Yarla, FU & 1 & 0.5 \\
\hline 30. & Pillai, FZ & 1 & 0.5 \\
\hline 31. & Arayar, GO & 1 & 0.5 \\
\hline 32. & Joardar, GW & 1 & 0.5 \\
\hline 33. & Velu, HE & 1 & 0.5 \\
\hline
\end{tabular}

The embeddedness and indirect social capital of knowledge sources are listed in Table D.2. The main pesticide supplier to the village, M Raj, has the highest number (15) coincident links. A farmer from the village, FQ Yarla, and an NGO representative, B Meka, have five outgoing knowledge links each that are coincident with their indirect social ties. Thus, these three individuals have the highest values of indirect social capital from knowledge delivery in the village. The importance of the pesticide dealer and the NGO representatives as sources of knowledge is clear: they hold the highest and the second-highest value of out-degree centrality in the village's knowledge network (see section 4.3 in chapter 4 for an elaborate discussion). They are the experts, the former in pesticides and the latter in NPM, who are approached by farmers for advice when they face severe problems in pest management. The third person with the highest social capital for knowledge delivery is the headman of a community. He is the farmer who works most closely with the NGO and acts as a main resource person of 
the NGO in the village. He has also travelled to different parts of India as an ambassador of NPM on behalf of the NGO's. In terms of innovation capacity, he is one of three farmers who successfully experimented with new NPM practices.

Table D.2 Embeddedness of outgoing knowledge links in indirect social ties 'undivided' networks

\begin{tabular}{|l|l|l|l|}
\hline & I & II & III \\
\hline & $\begin{array}{l}\text { Name of the } \\
\text { individual }\end{array}$ & $\begin{array}{l}\text { Number of outgoing links } \\
\text { coincident with indirect social ties }\end{array}$ & $\begin{array}{l}\text { Value of proportional } \\
\text { embeddedness }\end{array}$ \\
\hline 1. & $\begin{array}{l}\text { Raj, M., A-one } \\
\text { Fertilizers }\end{array}$ & 15 & 0.190 \\
\hline 2. & Yarla, FQ & 5 & 0.313 \\
\hline 3. & Meka, B. & 5 & 0.147 \\
\hline 4. & Velu, HE & 4 & 1 \\
\hline 5. & Lal, BJ & 3 & 1 \\
\hline 6. & Kota, DN & 3 & 1 \\
\hline 7. & Koli, ED & 2 & 1 \\
\hline 8. & Udit, IO & 2 & 1 \\
\hline 9. & Seri, AU & 2 & 0.5 \\
\hline 10. & Gorinta, EG & 2 & 0.4 \\
\hline 11. & Sapna, EW & 2 & 0.4 \\
\hline 12. & Seri, AW & 1 & 1 \\
\hline 13. & Subba, CD & 1 & 1 \\
\hline 14. & Jali, CR & 1 & 1 \\
\hline 15. & Naidu, CX & 1 & 1 \\
\hline 16. & Koli, EA & 1 & 1 \\
\hline 17. & Navya, ET & 1 & 1 \\
\hline 18. & Sapna, FD & 1 & 1 \\
\hline 19. & Yarla, FV & 1 & 1 \\
\hline 20. & Pillai, FZ & 1 & 1 \\
\hline 21. & Palam, HV & 1 & 1 \\
\hline 22. & Palam, HY & 1 & 1 \\
\hline 23. & Veena, IG & 1 & 1 \\
\hline 24. & Seri, AR & 1 & 0.5 \\
\hline 25. & Lal, BM & 1 & 0.5 \\
\hline 26. & Muthu, GD & 1 & 0.5 \\
\hline 27. & Joardar, GY & 1 & 0.5 \\
\hline 28. & Gowda, CP & 1 & 0.25 \\
\hline 29. & Sapna, FC & 1 & 0.25 \\
\hline 30. & Gowda, CL & 1 & 0.143 \\
\hline 31. & Pillai, GB & 1 & 0.111 \\
\hline & & & \\
\hline & & 1 \\
\hline
\end{tabular}




\section{D.2 Individual level results in the community of agriculturalists (the reduced networks of 2II nodes)}

Once again I start with individual embeddedness of incoming knowledge links in direct social ties. The results are very similar to those presented in Table 9.2 (chapter 9) for the undivided networks.

Table D.3 Individual embeddedness of incoming knowledge links in direct social ties - Punukula only

\begin{tabular}{|l|l|l|l|}
\hline & I & II & III \\
\hline & $\begin{array}{l}\text { Name of the } \\
\text { individual }\end{array}$ & $\begin{array}{l}\text { Number of incoming links } \\
\text { coincident with social ties }\end{array}$ & $\begin{array}{l}\text { Degree of } \\
\text { embeddedness }\end{array}$ \\
\hline 1. & Koli, ED & 3 & 0.75 \\
\hline 2. & Lal, BE & 2 & 1 \\
\hline 3. & Pillai, GC & 2 & 1 \\
\hline 4. & Gorinta, EG & 2 & 0.667 \\
\hline 5. & Lal, BM & 1 & 1 \\
\hline 6. & Subba, CE & 1 & 1 \\
\hline 7. & Chet, DL & 1 & 1 \\
\hline 8. & Koli, EA & 1 & 1 \\
\hline 9. & Koli, EB & 1 & 1 \\
\hline 10. & Navya, EL & 1 & 1 \\
\hline 11. & Sapna, FC & 1 & 1 \\
\hline 12. & Yarla, FU & 1 & 1 \\
\hline 13. & Yarla, FV & 1 & 1 \\
\hline 14. & Pillai, FZ & 1 & 1 \\
\hline 15. & Pillai, GA & 1 & 1 \\
\hline 16. & Krithi, HB & 1 & 1 \\
\hline 17. & Palam, HS & 1 & 1 \\
\hline 18. & Navya, ES & 1 & 0.5 \\
\hline 19. & Veena, IE & 1 & 0.5 \\
\hline 20. & Sapna, EW & 1 & 0.333 \\
\hline 21. & Naidu, CY & 1 & 0.25 \\
\hline & & & \\
\hline
\end{tabular}

Values of individual embeddedness of outgoing knowledge links in corresponding direct social ties are listed Table D.4. Once again, as expected, the results are similar to those listed for the 'undivided' network in Table 9.3. The few differences include the absence of the main pesticide supplier who had the highest social capital value for knowledge delivery in the 'undivided' network. The president of the village (HE Velu) and another farmer (BJ Lal) are the only two members of the network with highest 
number of 3 embedded links, and thus the highest values of social capital from their position in the social network as knowledge sources.

Table D.4 Individual embeddedness of outgoing knowledge links in direct social ties - Punukula only

\begin{tabular}{|l|l|l|l|}
\hline & I & II & III \\
\hline & $\begin{array}{l}\text { Name of the } \\
\text { individual }\end{array}$ & $\begin{array}{l}\text { Number of outgoing links } \\
\text { coincident with social ties }\end{array}$ & $\begin{array}{l}\text { Degree of } \\
\text { embeddedness }\end{array}$ \\
\hline 1. & Lal, BJ & 3 & 1 \\
\hline 2. & Velu, HE & 3 & 0.75 \\
\hline 3. & Koli, ED & 2 & 1 \\
\hline 4. & Sapna, FC & 2 & 0.667 \\
\hline 5. & Subba, CD & 1 & 1 \\
\hline 6. & Naidu, CX & 1 & 1 \\
\hline 7. & Koli, EA & 1 & 1 \\
\hline 8. & Sapna, EY & 1 & 1 \\
\hline 9. & Yarla, FV & 1 & 1 \\
\hline 10. & Pillai, FZ & 1 & 1 \\
\hline 11. & Muthu, GD & 1 & 1 \\
\hline 12. & Palam, HV & 1 & 1 \\
\hline 13. & Palam, HY & 1 & 1 \\
\hline 14. & Lal, BM & 1 & 0.5 \\
\hline 15. & Udit, IO & 1 & 0.5 \\
\hline 16. & Kota, DN & 1 & 0.333 \\
\hline 17. & Pillai, GA & 1 & 0.333 \\
\hline 18. & Gorinta, EG & 1 & 0.25 \\
\hline 19. & Sapna, EW & 1 & 0.2 \\
\hline 20. & Yarla, FQ & 1 & 0.0667 \\
\hline & & & \\
\hline & & 1 & 1 \\
\hline
\end{tabular}

Embeddedness values of knowledge flows in the village in indirect social ties are listed in Tables D.5 and D.6 below. Results are once again quite similar to the corresponding results for the undivided networks, except for the absence of the pesticide dealers and the NGO representatives.

Table D.5 Individual embeddedness of incoming knowledge links in indirect social ties in Punukula only

\begin{tabular}{|l|l|l|l|}
\hline & I & II & III \\
\hline & $\begin{array}{l}\text { Name of the } \\
\text { individual }\end{array}$ & $\begin{array}{l}\text { Number of incoming links } \\
\text { coincident with indirect social ties }\end{array}$ & $\begin{array}{l}\text { Degree of } \\
\text { embeddedness }\end{array}$ \\
\hline 1. & Koli, ED & 4 & 1 \\
\hline 2. & Lal, BE & 2 & 1 \\
\hline
\end{tabular}




\begin{tabular}{|l|l|l|l|}
\hline 3. & Pillai, GC & 2 & 1 \\
\hline 4. & Veena, IE & 2 & 1 \\
\hline 5. & Gorinta, EG & 2 & 0.667 \\
\hline 6. & Seri, AT & 2 & 0.5 \\
\hline 7. & Seri, AW & 1 & 1 \\
\hline 8. & Trupti, BA & 1 & 1 \\
\hline 9. & Lal, BM & 1 & 1 \\
\hline 10. & Lal, BN & 1 & 1 \\
\hline 11. & Subba, CA & 1 & 1 \\
\hline 12. & Subba, CB & 1 & 1 \\
\hline 13. & Subba, CE & 1 & 1 \\
\hline 14. & Koli, DY & 1 & 1 \\
\hline 15. & Koli, EA & 1 & 1 \\
\hline 16. & Koli, EB & 1 & 1 \\
\hline 17. & Sapna, EU & 1 & 1 \\
\hline 18. & Sapna, FD & 1 & 1 \\
\hline 19. & Nath, FG & 1 & 1 \\
\hline 20. & Yarla, FU & 1 & 1 \\
\hline 21. & Yarla, FV & 1 & 1 \\
\hline 22. & Pillai, FZ & 1 & 1 \\
\hline 23. & Pillai, GA & 1 & 1 \\
\hline 24. & Krithi, HB & 1 & 1 \\
\hline 25. & Velu, HD & 1 & 1 \\
\hline 26. & Velu, HL & 1 & 1 \\
\hline 27. & Palam, HS & 1 & 1 \\
\hline 28. & Veena, ID & 1 & 1 \\
\hline 29. & Naidu, CU & 1 & 0.5 \\
\hline 30. & Karai, DF & 1 & 0.5 \\
\hline 31. & Navya, EN & 1 & 0.5 \\
\hline 32. & Yeluri, IM & 1 & 0.333 \\
\hline 33. & Sapna, EW & 1 & 0.25 \\
\hline 34. & Selvi, FF & 1 & \\
\hline 35. & Naidu, CY & 1 & 133 \\
\hline & & & 1 \\
\hline
\end{tabular}

Table D.6 Individual embeddedness of outgoing knowledge links in indirect social ties in Punukula only

\begin{tabular}{|l|l|l|l|}
\hline & I & II & III \\
\hline & $\begin{array}{l}\text { Name of the } \\
\text { individual }\end{array}$ & $\begin{array}{l}\text { Number of outgoing links } \\
\text { coincident with indirect social ties }\end{array}$ & $\begin{array}{l}\text { Degree of } \\
\text { embeddedness }\end{array}$ \\
\hline 27. & Yarla, FQ & 5 & 0.333 \\
\hline
\end{tabular}




\begin{tabular}{|l|l|l|l|}
\hline 14. & Velu, HE & 4 & 1 \\
\hline 2. & Lal, BJ & 3 & 1 \\
\hline 6. & Kota, DN & 3 & 1 \\
\hline 8. & Koli, ED & 2 & 1 \\
\hline 18. & Udit, IO & 2 & 1 \\
\hline 19. & Seri, AU & 2 & 0.667 \\
\hline 22. & Gorinta, EG & 2 & 0.5 \\
\hline 25. & Sapna, EW & 2 & 0.4 \\
\hline 1. & Seri, AW & 1 & 1 \\
\hline 3. & Subba, CD & 1 & 1 \\
\hline 4. & Jali, CR & 1 & 1 \\
\hline 5. & Naidu, CX & 1 & 1 \\
\hline 7. & Koli, EA & 1 & 1 \\
\hline 9. & Navya, ET & 1 & 1 \\
\hline 10. & Sapna, FD & 1 & 1 \\
\hline 11. & Yarla, FV & 1 & 1 \\
\hline 12. & Pillai, FZ & 1 & 1 \\
\hline 13. & Muthu, GD & 1 & 1 \\
\hline 15. & Palam, HV & 1 & 1 \\
\hline 16. & Palam, HY & 1 & 1 \\
\hline 17. & Veena, IG & 1 & 1 \\
\hline 20. & Lal, BM & 1 & 0.5 \\
\hline 21. & Gowda, CL & 1 & 0.5 \\
\hline 23. & Pillai, GB & 1 & 0.5 \\
\hline 24. & Joardar, GY & 1 & 0.5 \\
\hline 26. & Sapna, FC & 1 & 0.333 \\
\hline & & & \\
\hline
\end{tabular}

\section{D.3 Individual embeddedness of knowledge flows in business network of all transactions (farm-inputs, crop-sale but not credit)}

The results are similar to those presented for the embeddedness of knowledge flows in the business network for pest control (Tables 9.7 and 9.8 in Chapter 9). The small differences include the fact that the main pesticide supplier to the village, M. Raj, is now also the person with the largest number of incoming links (Table D.7). As expected, he still tops the list for the largest number of knowledge links coincident with business ties (Table D.8). 
Table D.7 Individual embeddedness of incoming knowledge links in direct business network of all transactions except credit

\begin{tabular}{|l|l|l|l|}
\hline & I & II & III \\
\hline & Name of the individual & $\begin{array}{l}\text { Number of incoming knowledge } \\
\text { flows coincident with business ties }\end{array}$ & $\begin{array}{l}\text { Degree of } \\
\text { embeddedness }\end{array}$ \\
\hline 1. & Raj, M., A-one Fertilizers & 3 & 0.75 \\
\hline 2. & Suji, FM & 2 & 0.667 \\
\hline 3. & Joardar, GY & 2 & 0.4 \\
\hline 4. & Veena, IE & 2 & 0.4 \\
\hline 5. & Seri, AX & 1 & 1 \\
\hline 6. & Seri, AZ & 1 & 1 \\
\hline 7. & Subba, CD & 1 & 1 \\
\hline 8. & Gowda, CJ & 1 & 1 \\
\hline 9. & Jali, CR & 1 & 1 \\
\hline 10. & Sagi, EI & 1 & 1 \\
\hline 11. & Sapna, EX & 1 & 1 \\
\hline 12. & Sapna, EY & 1 & 1 \\
\hline 13. & Sapna, EZ & 1 & 1 \\
\hline 14. & Sapna, FA & 1 & 1 \\
\hline 15. & Sapna, FE & 1 & 1 \\
\hline 16. & Nath, FH & 1 & 1 \\
\hline 17. & Sree, FI & 1 & 1 \\
\hline 18. & Subbarao, FK & 1 & 1 \\
\hline 19. & Yarla, FR & 1 & 1 \\
\hline 20. & Muthu, GD & 1 & 1 \\
\hline 21. & Ray, GK & 1 & 1 \\
\hline 22. & Joardar, GX & 1 & 1 \\
\hline 23. & Velu, HC & 1 & 1 \\
\hline 24. & Velu, HF & 1 & 1 \\
\hline 25. & Velu, HG & 1 & 1 \\
\hline 26. & Velu, HK & 1 & 1 \\
\hline 27. & Palam, HW & 1 & 1 \\
\hline 28. & Palam, HY & 1 & 1 \\
\hline 29. & Palam, IB & 1 & 1 \\
\hline 30. & Veena, IF & 1 & 1 \\
\hline 31. & Veena, IG & 1 & 0.5 \\
\hline 32. & Yeluri, IN & 1 & \\
\hline 33. & Udit, IO & 1 & 1 \\
\hline 34. & Reddy, R., Rani Fertilizers & 1 & \\
\hline 35. & Seri, AR & 1 & 1 \\
\hline & & 1 & 1 \\
\hline & 1 & 1 \\
\hline
\end{tabular}




\begin{tabular}{|c|c|c|c|}
\hline 36. & Seri, AW & 1 & 0.5 \\
\hline 37. & Lal, BL & 1 & 0.5 \\
\hline 38. & Datla, BW & 1 & 0.5 \\
\hline 39. & Subba, CA & 1 & 0.5 \\
\hline 40. & Subba, CB & 1 & 0.5 \\
\hline 41. & Subba, CC & 1 & 0.5 \\
\hline 42. & Gowda, CM & 1 & 0.5 \\
\hline 43. & Pal, DB & 1 & 0.5 \\
\hline 44. & Goli, DK & 1 & 0.5 \\
\hline 45. & Kotla, DO & 1 & 0.5 \\
\hline 46. & Koli, DW & 1 & 0.5 \\
\hline 47. & Koli, EB & 1 & 0.5 \\
\hline 48. & Navya, EL & 1 & 0.5 \\
\hline 49. & Sapna, EU & 1 & 0.5 \\
\hline 50. & Subbarao, FJ & 1 & 0.5 \\
\hline 51. & Yarla, FU & 1 & 0.5 \\
\hline 52. & Arayar, GO & 1 & 0.5 \\
\hline 53. & Joardar, GW & 1 & 0.5 \\
\hline 54. & Velu, HE & 1 & 0.5 \\
\hline 55. & Velu, HH & 1 & 0.5 \\
\hline 56. & Veena, IJ & 1 & 0.5 \\
\hline 57. & Seri, AU & 1 & 0.333 \\
\hline 58. & Datla, BV & 1 & 0.333 \\
\hline 59. & Datla, BZ & 1 & 0.333 \\
\hline 60. & Subba, CE & 1 & 0.333 \\
\hline 61. & Chevula, $\mathrm{CH}$ & 1 & 0.333 \\
\hline 62. & Gowda, CP & 1 & 0.333 \\
\hline 63. & Kota, DN & 1 & 0.333 \\
\hline 64. & Navya, EN & 1 & 0.333 \\
\hline 65. & Navya, EP & 1 & 0.333 \\
\hline 66. & Navya, ES & 1 & 0.333 \\
\hline 67. & Nath, FG & 1 & 0.333 \\
\hline 68. & Tapi, FO & 1 & 0.333 \\
\hline 69. & Yarla, FX & 1 & 0.333 \\
\hline 70. & Yarla, FY & 1 & 0.333 \\
\hline 71. & Krithi, HB & 1 & 0.333 \\
\hline 72. & Yeluri, IM & 1 & 0.333 \\
\hline 73. & Lal, BN & 1 & 0.25 \\
\hline 74. & Gorinta, EG & 1 & 0.25 \\
\hline 75. & Yarla, FV & 1 & 0.25 \\
\hline
\end{tabular}




\begin{tabular}{|l|l|l|l|}
\hline 76. & Venu, GE & 1 & 0.25 \\
\hline 77. & Seri, AT & 1 & 0.2 \\
\hline 78. & Datla, BT & 1 & 0.2 \\
\hline 79. & Moti, CQ & 1 & 0.2 \\
\hline 80. & Selvi, FF & 1 & 0.143 \\
\hline
\end{tabular}

Table D.8 Individual embeddedness of outgoing knowledge links in direct business network of all transactions

\begin{tabular}{|l|l|l|l|}
\hline & I & II & III \\
\hline & Name of the individual & $\begin{array}{l}\text { Number of incoming } \\
\text { knowledge flows coincident } \\
\text { with business ties }\end{array}$ & $\begin{array}{l}\text { Degree of } \\
\text { embeddedness }\end{array}$ \\
\hline 1. & Raj, M., A-one Fertilizers & 74 & 0.91139 \\
\hline 2. & Meka, B., SECURE & 4 & 0.11765 \\
\hline 3. & Kasi, N., Ramana Fertilizers & 2 & 0.5 \\
\hline 4. & $\begin{array}{l}\text { Alluri, T., Laxman } \\
\text { Fertilisers }\end{array}$ & 1 & 1 \\
\hline 5. & Ruma, AO & 1 & 1 \\
\hline 6. & $\begin{array}{l}\text { Chiruvella, P., Victory } \\
\text { Pesticides }\end{array}$ & 1 & 0.33333 \\
\hline 7. & Rao, S., Simbha Fertilisers & 1 & 0.33333 \\
\hline 8. & Seri, AZ & 1 & 0.33333 \\
\hline 9. & Seri, AU & 1 & 0.25 \\
\hline 10. & Gowda, CL & 1 & 0.14286 \\
\hline 11. & Addala, A., SECURE & 1 & 0 \\
\hline
\end{tabular}

Network level results for the embeddedness of knowledge flows in business network of all transactions are listed in Table D.9 below. More knowledge flows are coincident with direct business ties than with indirect ones in this network, once again lending support to the result on the importance of user-producer (buyer-seller) interactions for knowledge flows.

Table D.9 Network level embeddedness of knowledge flows in the business network of all transactions (direct and indirect)

\begin{tabular}{|l|l|l|}
\hline Path length of business ties & $\begin{array}{l}\text { Number of embedded } \\
\text { knowledge flows }\end{array}$ & Embeddedness \\
\hline Direct business relations & 85 & 0.2576 \\
\hline Indirect business relations & 66 & 0.2000 \\
\hline
\end{tabular}




\section{Samenvatting}

Voor voortgaande economische groei en waarborging van duurzaamheid van de natuurlijke omgeving is het noodzakelijk dat bestaande technologieën worden vervangen door nieuwe, efficiëntere en schonere technologieën. Deze technologische successie is echter een ingewikkeld proces, want individuele technologieën zijn altijd ingebed in grotere sociaal-technische systemen. Complexiteit in deze systemen is een gevolg van onderlinge technologische, economische en sociale afhankelijkheden. Met andere woorden, complexiteit komt voort uit de wederzijdse afhankelijkheid tussen verschillende complementaire en substitueerbare technologieën en daarmee verweven routines en gewoonten van actoren die deze technologieën ontwikkelen en benutten, en meer in het algemeen uit de overlap tussen technologische, economische en sociale interactienetwerken. In dit onderzoek wordt deze complexiteit onder de loep genomen met gebruikmaking van instrumenten uit de sociale netwerkanalyse. In deel I van de dissertatie wordt een simulatiestudie van technologische innovatie gepresenteerd. De simulatieresultaten worden vervolgens in deel II gebruikt om het proces van technologiesuccessie in een Indiaas dorp te analyseren.

De simulatiestudie behelst een nieuwe manier van kijken naar de structuur van interacties in een sociaal-technisch systeem. Meer in het bijzonder wordt een systeem en het bijbehorende netwerk van onderliggende soorten kennis gezien als een entiteit met een centrum-perifere structuur. Systeemgedrag in het model kent drie duidelijk onderscheidbare fasen. Een nieuw systeem is vormloos in een vroeg ontwikkelingsstadium, wanneer verschillende nieuwe technologieën (of verschillende versies van een technologie) worden uitgeprobeerd. Tijdens dit initiële stadium is er geen duidelijke structuur waarin verschillende delen van het system georganiseerd zijn. Het tevoorschijn komen van een kern brengt het system uit zijn experimentele fase; er volgt een stadium van kennisopbouw rondom een klein aantal (dominante) kernelementen. Elementen in de kern staan met elkaar in verbinding door middel van een mechanisme van positieve feedback: veranderingen in één kernelement sturen versterkende signalen naar andere kernelementen. Deze positieve versterking geeft de kern dynamiek om innovatie te ondersteunen in perifere (complementaire) elementen van het system die direct of indirect in verband staan met een kernelement. Op deze manier zetten veranderingen in een technologie aan tot innovatie in andere wederzijds afhankelijke technologieën in plaats van deze te belemmeren, en dit maakt het gehele systeem 'dynamisch duurzaam'. Een derde fase vangt aan als het systeem volledig is opgebouwd en volgroeid. Simulatie-experimenten laten zien dat de stabiliteit van een volwassen systeem om uitdagingen van nieuwere radicale innovaties te kunnen weerstaan afhankelijk is van de kracht van de dominante kern. De kracht van de kern is een functie van de relatiedichtheid in een circulair proces, en van het vermogen van de kernelementen om nieuwe kennisinputs van sommige perifere elementen te ontvangen.

In het empirische deel worden simulatieresultaten met betrekking tot centrumperifere structuren gebruikt om vat te krijgen op innovatie in een Indiaas dorp. 
Gesteund door een initiatief voor plattelandsontwikkeling geleid door twee maatschappelijke organisaties, beëindigden de boeren in het dorp het gebruik van pesticiden en gingen zij over op 'Non-pesticidal Management' (NPM) van hun gewassen. Het dorp verwierf grote beroemdheid in nationale en regionale media als een bio-dorp dat zichzelf had verlost van pesticiden en schulden. Het onderzoek analyseert technologiesuccessie in het dorp, met gebruikmaking van nieuwe gegevens over de kennisinteracties van de boeren, uitwisselingen tussen bedrijven (inputs voor de landbouw, krediet, en gewasverkopen), en sociale netwerken (met familie en vrienden). In elk van de drie netwerken werden centrum-perifere structuren aangetroffen en kon een dominante kern worden bepaald. De toepassing van simulatieresultaten met betrekking tot de kracht van de kernen in de kennis-, bedrijfs-, en sociale netwerken leveren redelijke verklaringen op voor (achtereenvolgens) de manier waarop NPM-innovatie plaatsvond, de schuldenlast van de boeren, en de sociaal-politieke dynamiek.

Het proefschrift behandelt bovendien de overlap tussen de drie netwerken van het dorp. Het proefschrift beargumenteert dat het nuttig is om een locaal system op te vatten als een set van netwerken die ingebed zijn in een meeromvattende samenleving en economie. Coherentie in zo'n innovatiesysteem houdt de mogelijkheid in dat de betrokken actoren bij voortduring locale activiteiten ondernemen ter bevordering van een gewenste technologie, waarbij zij verschillende vormen van sociaal kapitaal mobiliseren waarover zij als individuen beschikken. Op deze manier wordt een gewenste technologie in de kennis-, goederen-, en sociaal-politieke domeinen zowel apart als gezamenlijk bevorderd (met overlap in twee of meer domeinen). Bijvoorbeeld, coherentie in de zin van duurzame actie in het kennisdomein impliceert dat een gewenste technologie gebruikt en ontwikkeld zal blijven worden nadat de donoren en de experts die het project hadden opgezet zijn vertrokken. Om dit te bereiken is het nodig dat de beheersing en het management van de technologie in de handen terechtkomt van de mensen die de technologie gebruiken (of willen gaan gebruiken) als onderdeel van hun productieproces. In het geval van NPM beargumenteer ik dat dit alleen kan gebeuren als boeren in staat zijn om voldoende sociaal kapitaal te mobiliseren voor kennis die relevant is voor een wijd verbreid gebruik en verdere ontwikkeling van NPM. In het eerste instantie, kan sociale kapitaal voor kennis bij boeren vanuit het oogpunt van structuur worden geobserveerd in de vorm van hun positie in het kennisnetwerk. Bijvoorbeeld, indien (enige van) de boeren met elkaar samenwerken en daarmee een geduchte kernstructuur vormen, kunnen zij duurzaamheid in het kennisdomein van een locaal innovatiesysteem bewerkstelligen.

Coherentie in het kennisdomein alléén is echter onvoldoende om de dynamische duurzaamheid van een locaal systeem zeker te stellen. We moeten aandacht geven aan andersoortige sociale en economische relaties waarin de kennisverbanden in zijn ingebed. Sommige kennisverbanden kunnen bijvoorbeeld worden geschraagd door sociale ondersteuning en vertrouwen, maar andere niet. In zulke gevallen komt er een andere vorm van sociaal kapitaal in het spel, die zich manifesteert als vertrouwen of 
samenhorigheid. Deze vorm van sociaal kapitaal voor kennis, een functie van het aantal vertrouwensrelaties die ook kennis doorgeven, werd slechts in zwakke mate aangetroffen in het geval van de NPM interventie in het dorp. Een hoger percentage kennisstromen zijn ingebed in directe bedrijfsrelaties. Dit gegeven onderstreept het belang van interacties tussen gebruiker en producent, welke een hoeksteen vormen van het raamwerk van innovatiesystemen. 


\section{ABOUT THE AUTHOR}

Saurabh Arora was born in New Delhi, India. He studied mechanical engineering at the National Institute of Technology, Surat, and the University of Minnesota, Minneapolis before joining the UNU-MERIT PhD programme in Economics and Policy Studies of Technical Change in September 2001. Since September 2007, he is working as an Assistant Professor in Technology and Development Studies at the Technische Universiteit, Eindhoven. He lives with louise and akalpa in the Netherlands. 